\title{
Exploring innovative nodal imaging and treatment strategies in breast cancer : a next step in patient tailored axillart treatment?
}

Citation for published version (APA):

Schipper, R. J. (2015). Exploring innovative nodal imaging and treatment strategies in breast cancer : a next step in patient tailored axillart treatment? [Doctoral Thesis, Maastricht University]. Datawyse / Universitaire Pers Maastricht. https://doi.org/10.26481/dis.20151113rs

Document status and date:

Published: 01/01/2015

DOI:

10.26481/dis.20151113rs

Document Version:

Publisher's PDF, also known as Version of record

Please check the document version of this publication:

- A submitted manuscript is the version of the article upon submission and before peer-review. There can be important differences between the submitted version and the official published version of record.

People interested in the research are advised to contact the author for the final version of the publication, or visit the DOI to the publisher's website.

- The final author version and the galley proof are versions of the publication after peer review.

- The final published version features the final layout of the paper including the volume, issue and page numbers.

Link to publication

\footnotetext{
General rights rights.

- You may freely distribute the URL identifying the publication in the public portal. please follow below link for the End User Agreement:

www.umlib.nl/taverne-license

Take down policy

If you believe that this document breaches copyright please contact us at:

repository@maastrichtuniversity.nl

providing details and we will investigate your claim.
}

Copyright and moral rights for the publications made accessible in the public portal are retained by the authors and/or other copyright owners and it is a condition of accessing publications that users recognise and abide by the legal requirements associated with these

- Users may download and print one copy of any publication from the public portal for the purpose of private study or research.

- You may not further distribute the material or use it for any profit-making activity or commercial gain

If the publication is distributed under the terms of Article $25 \mathrm{fa}$ of the Dutch Copyright Act, indicated by the "Taverne" license above, 


\title{
EXPLORING INNOVATIVE NODAL IMAGING
}

\author{
AND \\ TREATMENT STRATEGIES IN BREAST CANCER
}

A NEXT STEP IN PATIENT TAILORED AXILLARY TREATMENT? 
Parts of the research described in this thesis were financially supported by grants from Stichting St. Annadal, Stichting a Sister's Hope and Kankeronderzoeksfonds Limburg.

Cover: Caroline Cracco, www.cracco.nl

Lay-out: H.A.M.E. Wouters-Lenssen

Printed by: Datawyse, Universitaire Pers Maastricht ISBN:

(C) R. Schipper, 2015

All rights reserved. No part of this publication may be reproduced, stored in a retrieval system or transmitted in any form or by any means without permission of the author, or, when appropriate, of the publishers of the publications.

The publication of this thesis was financially supported by: Covidien, Catharina Hospital Eindhoven, Maastricht University Medical Center + . 


\section{EXPLORING INNOVATIVE NODAL IMAGING}

\section{AND \\ TREATMENT STRATEGIES IN BREAST CANCER}

A NEXT STEP IN PATIENT TAILORED AXILLARY TREATMENT?

PROEFSCHRIFT

ter verkrijging van de graad van doctor

aan de Universiteit Maastricht,

op gezag van de Rector Magnificus, Prof. dr. L.L.G. Soete,

volgens het besluit van het College van Decanen,

in het openbaar te verdedigen op

op vrijdag 13 november 2015 om 14.00 uur

door

Robert-Jan Schipper 
Promotor

Prof. dr. R.G.H. Beets-Tan

\section{Co-promotores}

Dr. M.L. Smidt

Dr. M.B.I. Lobbes

\section{Beoordelingscommissie}

Prof. dr. G.L. Beets (voorzitter)

Prof. dr. M. van Goethem, Antwerp University

Prof. dr. M.F. von Meyenfeldt

Prof. dr. E.J. Rutgers, UvA

Prof. dr. H.J. Rutten

Dr. R.M. Mann, UMC Radboud Nijmegen 


\section{Contents}

Chapter 1 General introduction and outline of the thesis

Chapter 2 Axillary ultrasound for preoperative nodal staging in

breast cancer patients: is it of added value?

Breast 2013;22:1108-13

Chapter 3 The diagnostic performance of dedicated axillary T2- and

diffusion-weighted MRI for nodal staging in breast cancer

Radiology 2015;275:345-55

Chapter $4 \quad$ Noninvasive nodal staging in patients with breast cancer using

gadofosveset-enhanced magnetic resonance imaging: a

feasibility study

Invest Radiol 2013;48:134-9

Chapter 5 Noninvasive nodal restaging in clinically node positive

breast cancer patients after neoadjuvant systemic therapy:

a systematic review

Eur J Radiol 2015;84:41-7

Chapter 6 A model to predict pathologic complete response of axillary

lymph nodes to neoadjuvant chemo(immuno)therapy in patients with clinically node positive breast cancer

Clin Breast Cancer 2014;14:315-22

Chapter 7 The diagnostic performance of gadofosveset-enhanced axillary

MRI for nodal (re-)staging in breast cancer patients:

can the initial promising results be reproduced?

Chapter 8 The impact of the pathological lymph node status on adjuvant systemic treatment recommendations in clinically node negative breast cancer patients

Breast Cancer Res Treat 2014;143:469-76

Chapter 9 Three-dimensional breast radiotherapy and the elective radiation dose at the sentinel lymph node site in breast cancer Ann Surg Oncol. 2015 Feb 24

Chapter 10 Discussion and future perspective

Summary

Samenvatting

Valorisation

List of publications

Dankwoord 



\section{Chapter 1}

Introduction 
Chapter 1 


\section{Introduction}

Breast cancer is the most common cancer among women worldwide, with 1.38 million newly diagnosed patients in $2008^{1}$. Breast cancer incidence in the Netherlands increased to 14,326 new patients in $2013^{2}$. Due to improved multidisciplinary treatment, survival rates of breast cancer patients have improved and are still increasing, with a current 5 -year overall survival rate of $88 \%^{3}$. This results in the high prevalence of 104.213 breast cancer patients In the Netherlands in $2013^{2}$.

Nowadays, the treatment of patients with breast cancer consists of resection of the primary tumor and one or more axillary lymph nodes, in combination with hormonal-, chemo-, immuno- and/or radiotherapy (if indicated) ${ }^{4}$. The quality of life of breast cancer survivors is affected by morbidity caused by this extensive treatment ${ }^{5-8}$. More research is warranted to further individualize breast cancer treatment, thus improving the quality of life of breast cancer patients without compromising overall survival.

For a long time the standard procedure to assess axillary lymph node status in patients with breast cancer was an axillary lymph node dissection (ALND), a surgical procedure in which all axillary lymph nodes were removed ${ }^{9}$. Of all patients undergoing an ALND, only $30-40 \%$ had pathologically proven lymph node metastases ${ }^{10}$. In the remaining $60-70 \%$ of the patients, the ALND resulted in neither consequences for adjuvant treatment nor improvement of overall survival. They were unnecessarily exposed to the risk of short- and long-term morbidity of the procedure. Most reported complications are seroma (15-75\%), upper extremity lymph edema (20\%), nerve injury (55-75\%) and reduced shoulder function $(16 \%)^{8}$. The psychological impact of lymphedema has been described to be as distressing as the initial breast cancer diagnosis. Patients with breast cancer related lymphedema have a lower quality of life, a higher level of anxiety or depression, a higher likelihood of chronic pain and fatigue, and greater difficulty in functioning socially and sexually compared to breast cancer patients without lymphedema ${ }^{11}$.

To reduce the number of patients that unnecessarily underwent an ALND (i.e. in absence of lymph node metastases), the sentinel lymph node biopsy (SLNB) was introduced. This procedure is based on the pattern of lymphatic drainage from the tumor to one or more regional lymph nodes, assuming that if these lymph nodes do not contain tumor cells, all other regional lymph nodes are free of tumor cells as well ${ }^{12}$. In case one or more sentinel lymph nodes contain a metastasis, a completion ALND was indicated. High accuracy, low false-negative rates and safety of the SLNB procedure were proven by several studies and randomized controlled trials ${ }^{13-18}$. Complications known from ALND still occur after SLNB, though less frequently. Most reported are 
seroma, hematoma, lymphedema, numbness, tingling and pain ${ }^{6}$. Nonetheless, severe lymphedema still occurs in up to $8 \%$ of these patients after 3 years ${ }^{19}$.

Shortly after the SLNB implementation, pre-operative axillary ultrasound (with tissue sampling, if indicated) was implemented in order to identify patients with axillary lymph node metastases. Axillary ultrasound in combination with tissue sampling proved to be reliable in detecting lymph node metastases with a median sensitivity of $79.4 \%$, and a median specificity of $100 \%{ }^{20}$. In patients with preoperative pathologically proven lymph node metastases the SLNB procedure could be skipped and an ALND could be performed immediately ${ }^{21}$. This approach prevents half of patients from having to undergo two operations (SLNB, followed by ALND) ${ }^{20,22}$.

It would be a step forward if we would be able to identify the true node negative and true node positive patient as accurate as possible with noninvasive imaging techniques, thereby avoiding the risk of the morbidity of the SLNB and ALND procedures. Current imaging modalities, including preoperative breast magnetic resonance imaging (MRI), positron-emission tomography (PET) or computer tomography (CT) are considered to be insufficient in terms of diagnostic performance to serve as an alternative for $\mathrm{SLNB}^{23-26}$. With respect to $\mathrm{MRI}$, previous investigators mainly evaluated axillary lymph nodes visible on (preoperative) breast MRI instead of using dedicated, high spatial resolution axillary MRI sequences ${ }^{27-31}$. It has been shown that the use of dedicated axillary MR imaging protocols improves the accuracy of MRI in nodal staging ${ }^{24,32,33}$. The pooled data analysis of five studies using USPIO-enhanced MRI showed a mean sensitivity and specificity of $98 \%$ and $96 \%$ respectively. However the USPIO contrast is not approved by the Food and Drug Administration and therefore not available for routine clinical use ${ }^{34}$. Publications on contrast-enhanced MRI for lymph node staging in patients with rectal cancer using gadofosveset as contrast agent show promising results $^{35,36}$. Gadofosveset-enhanced MRI showed a high negative predictive value (NPV) for nodal metastases detection equal to that of USPIO-enhanced $\mathrm{MRI}^{36}$. Hypothetically, gadofosveset-enhanced axillary MRI could provide useful information about nodal status in patients with breast cancer.

However, it was believed that if small metastases in axillary lymph nodes, hard to detect with noninvasive staging techniques, were left behind in the patient), regional recurrence an overall survival were compromised. This paradigm was overthrown by several recently published randomized trails, such as the ACOSOG Z0011, which compared a completion ALND with no further axillary treatment in women with T1-2 breast cancer and one or two sentinel lymph node metastases treated by breast conserving therapy. After a median follow-up of 6.3 years, a 5 -year survival of $91.8 \%$ for the ALND arm and $92.5 \%$ for the SLNB arm was reported in this study ${ }^{37}$. In the control arm, $27.3 \%$ of the patients had additional axillary lymph node metastases removed by 
the completion ALND, implicating that in the study arm in $27.3 \%$ of the patients axillary lymph node metastases were left behind, without impact on regional and distant control. The knowledge that the detection and treatment of every single lymph node metastasis in patients with breast cancer is not as crucial as previously assumed, creates opportunities for research on axillary lymph node staging through noninvasive imaging studies.

Systemic therapy is increasingly administered in neoadjuvant setting: In order to downsize the primary tumor, and possibly facilitate breast conserving therapy or increase the chances of safer resection margins ${ }^{38-40}$. Apart from the primary tumor response, lymph nodes metastases can respond as well. Examination of ALND specimens showed that pathological complete response $(p C R)$ of axillary lymph node metastases due to neoadjuvant systemic therapy was achieved in $20-42 \%{ }^{41-44}$. Since axillary $\mathrm{pCR}$ is a strong predictor of disease-free survival ALND might be omitted in these patients ${ }^{45}$. Nonetheless, it appears challenging to identify these patients with axillary pCR with help of minimal or non-invasive staging strategies ${ }^{19}$.

In conclusion, the quality of life of breast cancer survivors has significantly improved in the past 20 years with the introduction of the SLNB and the possibility to omit completion ALND after detection of one or two metastasis in the sentinel lymph nodes in a selected group of patients with breast cancer ${ }^{7,8}$. A continuing drawback of nodal staging with SLNB is that a negative SLNB does not contribute to the survival, nor affect the indication of adjuvant therapy. The same applies to the (completion) ALND, especially if the ALND-specimen does not contain (additional) metastases. Nevertheless, patients are at risk of complications of these surgical procedures ${ }^{19}$. Recent novel insights create opportunities to investigate whether a noninvasive modern imaging technology can improve axillary lymph node staging in patients with breast cancer. 


\section{Aims and outline of this thesis}

The overall focus of this research is to study the opportunities of a more tailored made treatment plan for each breast cancer patient, in order to minimize the morbidity of the breast cancer survivor without compromising overall survival.

Chapter $\mathbf{2}$ evaluates axillary ultrasound as imaging modality to differentiate between patients with limited axillary disease (defined as pN1) and advanced axillary disease (defined as pN2 or pN3). In Chapter 3 the diagnostic performance of non-enhanced, axillary T2-weighted and diffusion weighted MR imaging for nodal staging in a population of newly diagnosed breast cancer patients is investigated. In Chapter 4, a feasibility study on use of a dedicated contrast enhanced axillary MRI to identify patients with no (macro) metastatic lymph nodes is described.

A systematic review on noninvasive nodal restaging techniques of the axillary lymph node in initial node positive breast cancer patients after neoadjuvant systemic treatment is presented in Chapter 5. In Chapter 6 the accuracy of a nomogram is presented based on standard available parameters of clinically node positive patients, to identify the patient with clinically node positive breast cancer who are likely to achieve a complete axillary response to neoadjuvant systemic therapy.

In Chapter 7, the diagnostic performance of a gadofosveset-enhanced axillary MRI for nodal staging and restaging in a second cohort of patients with breast cancer was investigated. Since small metastases could be missed by imaging techniques for nodal staging, and thereby might influence the decision to recommend adjuvant systemic therapy, the impact of unknowing of the true pathological lymph node status on adjuvant systemic treatment recommendations was simulated in Chapter 8. In Chapter 9 the incidental radiation dose delivered to the site of the sentinel lymph node in patients treated with lumpectomy, followed by whole breast radiotherapy, using modern radiotherapy planning techniques was investigated. Small metastases in the sentinel lymph node that left behind are probably sterilized by radiotherapy.

Finally, in Chapter 10, the most important findings of this thesis are discussed and recommendations for future research are suggested. 


\section{References}

1. Jemal A, Bray F, Center MM, Ferlay J, Ward E, Forman D. Global cancer statistics. CA Cancer J Clin 2011; 61:69-90.

2. Dutch Cancer Registery, C.C.C.t.N.C. [cited 20142 december]; Available from: http://www.cijfersoverkanker.nl.

3. Howlader N, Noone AM, Krapcho M, Garshell J, Neyman N, Altekruse SF, Kosary CL, Yu M, Ruhl J, Tatalovich Z, Cho H, Mariotto A, Lewis DR, Chen HS, Feuer EJ, Cronin KA. SEER Cancer Statistics Review, 1975-2010, National Cancer Institute. 2013; Available from: http://seer.cancer.gov/csr/1975_2010/.

4. Senkus E, Kyriakides S, Penault-Llorca F, Poortmans P, Thompson A, Zackrisson S, Cardoso F; ESMO Guidelines Working Group. Primary breast cancer: ESMO Clinical Practice Guidelines for diagnosis, treatment and follow-up. Ann Oncol 2013;24 Suppl 6:vi7-23.

5. Schulze T, Mucke J, Markwardt J, Schlag PM, Bembenek A. Long-term morbidity of patients with early breast cancer after sentinel lymph node biopsy compared to axillary lymph node dissection. J Surg Oncol 2006;93:109-19.

6. Schijven MP, Vingerhoets AJ, Rutten HJ, Nieuwenhuijzen GA, Roumen RM, van Bussel ME, Voogd AC. Comparison of morbidity between axillary lymph node dissection and sentinel node biopsy. Eur J Surg Oncol 2003;29:341-50.

7. Liu CQ, Guo Y, Shi JY, Sheng Y. Late morbidity associated with a tumour-negative sentinel lymph node biopsy in primary breast cancer patients: a systematic review. Eur J Cancer 2009;45:1560-8.

8. Ashikaga T, Krag DN, Land SR, Julian TB, Anderson SJ, Brown AM, Skelly JM, Harlow SP, Weaver DL, Mamounas EP, Costantino JP, Wolmark N. Morbidity results from the NSABP B-32 trial comparing sentinel lymph node dissection versus axillary dissection. J Surg Oncol 2010;102:111-8.

9. Halsted WS. I. A Clinical and Histological Study of certain Adenocarcinomata of the Breast: and a Brief Consideration of the Supraclavicular Operation and of the Results of Operations for Cancer of the Breast from 1889 to 1898 at the Johns Hopkins Hospital. Ann Surg 1898;28:557-76.

10. Fisher B, Jeong JH, Anderson S, Bryant J, Fisher ER, Wolmark N. Twenty-five-year follow-up of a randomized trial comparing radical mastectomy, total mastectomy, and total mastectomy followed by irradiation. N Engl J Med 2002;347:567-75.

11. Shih YC, Xu Y, Cormier JN, Giordano S, Ridner SH, Buchholz TA, Perkins GA, Elting LS. Incidence, treatment costs, and complications of lymphedema after breast cancer among women of working age: a 2-year follow-up study. J Clin Oncol 2009;27:2007-14.

12. Giuliano AE, Dale PS, Turner RR, Morton DL, Evans SW, Krasne DL. Improved axillary staging of breast cancer with sentinel lymphadenectomy. Ann Surg 1995;222:394-9.

13. Kim $T$, Giuliano $A E$, Lyman $G H$. Lymphatic mapping and sentinel lymph node biopsy in early-stage breast carcinoma: a metaanalysis. Cancer 2006;106:4-16.

14. Krag DN, Anderson SJ, Julian TB, Brown AM, Harlow SP, Ashikaga T, Weaver DL, Miller BJ, Jalovec LM, Frazier TG, Noyes RD, Robidoux A, Scarth HM, Mammolito DM, McCready DR, Mamounas EP, Costantino JP, Wolmark N, National Surgical Adjuvant Breast Bowel Project. Technical outcomes of sentinel-lymphnode resection and conventional axillary-lymph-node dissection in patients with clinically node-negative breast cancer: results from the NSABP B-32 randomised phase III trial. Lancet Oncol 2007;8:881-8.

15. Krag DN, Anderson Sj, Julian TB, Brown AM, Harlow SP, Costantino JP, Ashikaga T, Weaver DL, Mamounas EP, Jalovec LM, Frazier TG, Noyes RD, Robidoux A, Scarth HM, Wolmark N. Sentinel-lymph-node resection compared with conventional axillary-lymph-node dissection in clinically node-negative patients with breast cancer: overall survival findings from the NSABP B-32 randomised phase 3 trial. Lancet Oncol 2010;11:927-33.

16. Mansel RE, Fallowfield L, Kissin M, Goyal A, Newcombe RG, Dixon JM, Yiangou C, Horgan K, Bundred N, Monypenny I, England D, Sibbering M, Abdullah TY, BarrL, Chetty U, Sinnett DH, Fleissig A, Clarke D, Ell PJ. Randomized multicenter trial of sentinel node biopsy versus standard axillary treatment in operable breast cancer: the ALMANAC Trial. J Natl Cancer Inst 2006;98:599-609.

17. van der Ploeg IM, Nieweg OE, van Rijk MC, Valdes Olmos RA, Kroon BB. Axillary recurrence after a tumour-negative sentinel node biopsy in breast cancer patients: A systematic review and meta-analysis of the literature. Eur J Surg Oncol 2008;34:1277-84. 
18. Veronesi U, Paganelli G, Viale G, Luini A, Zurrida S, Galimberti V, Intra M, Veronesi P, Robertson C, Maisonneuve P, Renne G, De Cicco C, De Lucia F, Gennari R. A randomized comparison of sentinel-node biopsy with routine axillary dissection in breast cancer. N Engl J Med 2003;349:546-53.

19. DiSipio T, Rye S, Newman B, Hayes $S$. Incidence of unilateral arm lymphoedema after breast cancer: a systematic review and meta-analysis. Lancet Oncol 2013;14:500-15.

20. Houssami N, Ciatto S, Turner RM, Cody HS 3rd, Macaskill P. Preoperative ultrasound-guided needle biopsy of axillary nodes in invasive breast cancer: meta-analysis of its accuracy and utility in staging the axilla. Ann Surg 2011;254:243-51.

21. Bonnema J, van Geel AN, van Ooijen B, Mali SP, Tjiam SL, Henzen-Logmans SC, Schmitz PI, Wiggers T. Ultrasound-guided aspiration biopsy for detection of nonpalpable axillary node metastases in breast cancer patients: new diagnostic method. World J Surg 1997;21:270-4.

22. Diepstraten SC, Sever AR, Buckens CF, Veldhuis WB, van Dalen T, van den Bosch MA, Mali WP, Verkooijen HM. Value of preoperative ultrasound-guided axillary lymph node biopsy for preventing completion axillary lymph node dissection in breast cancer: a systematic review and meta-analysis. Ann Surg Oncol 2014;21:51-9.

23. Mortellaro VE, Marshall J, Singer L, Hochwald SN, Chang M, Copeland em, Grobmyer SR. Magnetic resonance imaging for axillary staging in patients with breast cancer. J Magn Reson Imaging 2009;30: 309-12.

24. Kvistad KA, Rydland J, Smethurst HB, Lundgren S, Fjosne HE, Haraldseth O. Axillary lymph node metastases in breast cancer: preoperative detection with dynamic contrast-enhanced MRI. Eur Radiol, 2000. 10(9): p. 1464-71.

25. Cooper KL, Harnan S, Meng Y, Ward SE, Fitzgerald P, Papaioannou D, Wyld L, Ingram C, Wilkinson ID, Lorenz E. Positron emission tomography (PET) for assessment of axillary lymph node status in early breast cancer: A systematic review and meta-analysis. Eur J Surg Oncol 2011;37:187-98.

26. Harnan SE, Cooper KL, Meng Y, Ward SE, Fitzgerald P, Papaioannou D, Ingram C, Lorenz E, Wilkinson ID, Wyld L. Magnetic resonance for assessment of axillary lymph node status in early breast cancer: a systematic review and meta-analysis. Eur J Surg Oncol 2011;37:928-36.

27. Scaranelo AM, Eiada R, Jacks LM, Kulkarni SR, Crystal P. Accuracy of unenhanced MR imaging in the detection of axillary lymph node metastasis: study of reproducibility and reliability. Radiology 2012;262: 425-34.

28. He N, Xie C, Wei W, Pan C, Wang W, Lv N, Wu P. A new, preoperative, MRI-based scoring system for diagnosing malignant axillary lymph nodes in women evaluated for breast cancer. Eur J Radiol 2012;81: 2602-12.

29. Fornasa F, Nesoti MV, Bovo C, Bonavina MG. Diffusion-weighted magnetic resonance imaging in the characterization of axillary lymph nodes in patients with breast cancer. J Magn Reson Imaging 2012;36: 858-64.

30. Luo N, Su D, Jin G, Liu L, Zhu X, Xie D, Liu Y. Apparent diffusion coefficient ratio between axillary lymph node with primary tumor to detect nodal metastasis in breast cancer patients. J Magn Reson Imaging, 2013;38:824-8.

31. Kamitani T, Hatakenaka M, Yabuuchi H, Matsuo $Y$, Fujita N, Jinnouchi $M$, Nagao M, Shirahane K, Tokunaga $\mathrm{E}$, Honda $\mathrm{H}$. Detection of axillary node metastasis using diffusion-weighted $\mathrm{MRI}$ in breast cancer. Clin Imaging 2013;37:56-61.

32. Baltzer PA, Dietzel M, Burmeister HP, Zoubi R, Gajda M, Camara O, Kaiser WA. Application of MR mammography beyond local staging: is there a potential to accurately assess axillary lymph nodes? evaluation of an extended protocol in an initial prospective study. AJR Am J Roentgenol 2011;196: W641-7.

33. Luciani A, Dao TH, Lapeyre M, Schwarzinger M, Debaecque C, Lantieri L, Revelon G, Bouanane M, Kobeiter $\mathrm{H}$, Rahmouni A. Simultaneous bilateral breast and high-resolution axillary MRI of patients with breast cancer: preliminary results. AJR Am J Roentgenol 2004;182:1059-67.

34. Cooper KL, Meng Y, Harnan S, Ward SE, Fitzgerald P, Papaioannou D, Wyld L, Ingram C, Wilkinson ID, Lorenz E., Positron emission tomography (PET) and magnetic resonance imaging (MRI) for the assessment of axillary lymph node metastases in early breast cancer: systematic review and economic evaluation. Health Technol Assess 2011;15:iii-iv, 1-134. 
35. Lahaye GLB, Engelen SME, Voth M, Leiner T, Lambregts DMJ, Beets-Tan RGH. Gadovosfeset Trisodium (Vasovist) Enhanced MR Lymphnode detection: Initial Observations. The Open Magnetic Resonance Journal 2009;2:28-32.

36. Lambregts DM, Beets GL, Maas M, Kessels AG, Bakers FC, Cappendijk VC, Engelen SM, Lahaye MJ, de Bruine AP, Lammering G, Leiner T, Verwoerd JL, Wildberger JE, Beets-Tan RG. Accuracy of gadofosvesetenhanced MRI for nodal staging and restaging in rectal cancer. Ann Surg 2011;253:539-45.

37. Giuliano AE, Hunt KK, Ballman KV, Beitsch PD, Whitworth PW, Blumencranz PW, Leitch AM, Saha S, McCall LM, Morrow M. Axillary dissection vs no axillary dissection in women with invasive breast cancer and sentinel node metastasis: a randomized clinical trial. JAMA 2011;305:569-75.

38. Fisher B, Bryant J, Wolmark N, Mamounas E, Brown A, Fisher ER, Wickerham DL, Begovic M, DeCillis A, Robidoux A, Margolese RG, Cruz AB Jr, Hoehn JL, Lees AW, Dimitrov NV, Bear HD. Effect of preoperative chemotherapy on the outcome of women with operable breast cancer. J Clin Oncol 1998;16:2672-85.

39. Kaufmann M, von Minckwitz G, Mamounas EP, Cameron D, Carey LA, Cristofanilli M, Denkert C, Eiermann W, Gnant M, Harris JR, Karn T, Liedtke C, Mauri D, Rouzier R, Ruckhaeberle E, SemiglazovV, Symmans WF, Tutt A, Pusztai L. Recommendations from an international consensus conference on the current status and future of neoadjuvant systemic therapy in primary breast cancer. Ann Surg Oncol 2012;19:1508-16.

40. van der Hage JA, van de Velde CJ, Julien JP, Tubiana-Hulin M, Vandervelden C, Duchateau L. Preoperative chemotherapy in primary operable breast cancer: results from the European Organization for Research and Treatment of Cancer trial 10902. J Clin Oncol 2001;19:4224-37.

41. Alvarado R, Yi M, Le-Petross H, Gilcrease M, Mittendorf EA, Bedrosian I, Hwang RF, Caudle AS, Babiera GV, Akins JS, Kuerer HM, Hunt KK. The role for sentinel lymph node dissection after neoadjuvant chemotherapy in patients who present with node-positive breast cancer. Ann Surg Oncol 2012;19: 3177-84.

42. Koolen BB, Valdes Olmos RA, Wesseling J, Vogel WV, Vincent AD, Gilhuijs KG, Rodenhuis S, Rutgers EJ, Vrancken Peeters MJ. Early assessment of axillary response with (1)(8)F-FDG PET/CT during neoadjuvant chemotherapy in stage II-III breast cancer: implications for surgical management of the axilla. Ann Surg Oncol 2013;20:2227-35.

43. Rouzier R, Extra JM, Klijanienko J, Falcou MC, Asselain B, Vincent-Salomon A, Vielh P, Bourstyn E. Incidence and prognostic significance of complete axillary downstaging after primary chemotherapy in breast cancer patients with T1 to T3 tumors and cytologically proven axillary metastatic lymph nodes. J Clin Oncol 2002;20:1304-10.

44. Straver ME, Rutgers EJ, Russell NS, Oldenburg HS, Rodenhuis S, Wesseling J, Vincent A, Peeters MT. Towards rational axillary treatment in relation to neoadjuvant therapy in breast cancer. Eur J Cancer 2009;45:2284-92.

45. von Minckwitz G, Untch M, Blohmer JU, Costa SD, Eidtmann H, Fasching PA, Gerber B, Eiermann W, Hilfrich J, Huober J, Jackisch C, Kaufmann M, Konecny GE, Denkert C, Nekljudova V, Mehta K, Loibl S. Definition and impact of pathologic complete response on prognosis after neoadjuvant chemotherapy in various intrinsic breast cancer subtypes. J Clin Oncol 2012;30:1796-804. 


\section{Chapter}

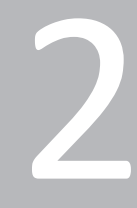

Axillary ultrasound for preoperative nodal staging in breast cancer patients: Is it of added value?

RJ. Schipper*

L.M. van Roozendaal*

B. de Vries

R.M. Pijnappel

R.G.H. Beets-Tan

M.B.I. Lobbes

M.L. Smidt

* Both authors contributed equally to this manuscript

Breast 2013;22:1108-1113 


\section{Abstract}

\section{Background}

New insights show that an axillary lymph node dissection (ALND) may not always be indicated for metastases detected by ultrasound (pathologically proven). This study investigated whether axillary ultrasound accurately predicts pN0, pN1 and pN2-pN3 status.

\section{Methods}

Data were retrospectively collected from all consecutive patients with invasive breast cancer who underwent (primary) surgery between 2008 and 2012. False negative percentages and negative predictive values (NPVs) for sonographic nodal staging were calculated for all patients and again for cT1-2 patients treated by breast conserving therapy (BCT).

\section{Results}

A total of 577 axillary ultrasounds were included. After negative ultrasound findings (cN0), pathology showed pN2-pN3 disease in $4.4 \%$ of these cases, with an NPV of 95.5\% (93.4-97.1\%). When cN1 (1-3 suspicious nodes) was predicted, pathology showed pN2-pN3 disease in $41.2 \%$, with an NPV of $58.5 \%(44.2-71.5 \%)$.

In the subgroup of patients with cT1-2 breast cancer that were treated by BCT, pathology showed pN2-pN3 disease in 2.3\% after negative ultrasound findings (cNO), with an NPV of $97.7 \%$ (94.9-99.0\%). When cN1 was predicted $(n=12)$, pathology showed pN2-pN3 disease in $50.0 \%$, with an NPV of $50.0 \%$ (22.3-77.9\%). A direct ALND was performed in these $12 \mathrm{cN} 1$ cases; pathology showed six patients with pN1 (three patients with one and three with two macrometastases) and six with pN2-pN3 disease $(4,5,11,13,16$ or 22 macrometastases, respectively).

\section{Conclusion}

In conclusion, a negative axillary ultrasound generally excludes the presence of pN2-pN3 disease. An axillary ultrasound cannot accurately differentiate between pN1 and pN2-pN3. It could be argued that the standard performance of an axillary ultrasound in breast cancer patients is questionable; multidisciplinary discussion could guide decisions on the use of axillary ultrasound for the individual patient. 


\section{Introduction}

The sentinel lymph node biopsy (SLNB) is regarded as the standard surgical nodal staging technique for clinically node negative breast cancer patients. In the absence of a sentinel lymph node (SLN) metastasis, the SLNB has replaced routine axillary lymph node dissection (ALND) for staging axillary lymph nodes ${ }^{1}$, thereby reducing the number of unnecessary ALNDs as well as the morbidity rate (seroma, infection, lymphedema, arm- and shoulder pain, nerve injury) ${ }^{2,3}$. About $30-40 \%$ of newly diagnosed breast cancer patients have nodal metastases ${ }^{4}$. Preoperative axillary ultrasound is widely used for assessing the clinical nodal status in breast cancer. If the patient's axillary evaluation is negative, an SLNB is performed for further staging. If a suspicious lymph node is detected with ultrasound, this node will then be sampled and if pathology shows a metastasis, the SLNB is omitted and an ALND is indicated ${ }^{5}$. The ALND can then be performed simultaneously with the tumor excision. This approach prevents $19.8 \%$ of patients from having to undergo an additional operation ${ }^{6}$.

Several retrospective studies, which included patients treated with mastectomy and breast conserving surgery, assessed the differences in recurrence and survival rates for SLN-positive patients treated by SLNB alone compared to patients who received an SLNB with completion ALND ${ }^{7-11}$. Interestingly, no clinically relevant benefit was demonstrated for completion ALND, although patient selection might be a confounding factor. More evidence can be deduced from several randomized controlled trials. These trials have shown that either performing axillary radiotherapy ${ }^{12}$, or omitting further axillary treatment following a positive sentinel lymph node $e^{6,13}$, does not compromise overall survival, nor disease control. The sentinel lymph node positive breast cancer patients included in these trials had a clinically node negative status by physical examination. One of these trials, the American College of Surgeons Oncology Group (ACOSOG) Z0011 trial demonstrated that omitting the completion ALND after the detection of 1-2 SLN metastases provided neither worsened regional control nor an inferiority of survival in clinically node negative breast cancer patients (cT1-2) treated by breast conserving therapy (BCT). In $97 \%$ of the patients, adjuvant systemic therapy was administered and the whole breast was radiated (with possible tangential radiation overlap to the axilla). As the completion ALND specimen contained additional nodal metastases in $27 \%$ of the patients in the control arm, patients randomized to the SLNBalone arm were likely to have important residual non-SLN metastases that were not removed by surgery ${ }^{13}$. This suggests that not all non-SLN metastases develop into clinically detectable disease.

This raises the question what the added value is of performing a preoperative evaluation of the axilla by ultrasound, and whether ALND is indicated for every metastasis detected by ultrasound. Consequently, preoperative axillary ultrasound might only be useful if it can differentiate between patients with limited axillary disease (defined as pN1) and those with advanced axillary disease (defined as pN2 or pN3). This 
would prevent the need for direct ALNDs in patients with limited nodal disease and facilitate the direct performance of ALNDs in patients with advanced nodal disease.

The aim of this retrospective study was to determine whether preoperative axillary ultrasound can distinguish between pN0, pN1 and pN2-pN3 disease.

\section{Materials and methods}

\section{Study population and data collection}

Due to the retrospective nature of this study, a certified medical ethics committee waived the requirement of informed consent. Between 2008 and 2012, all consecutive patients with operable primary invasive breast cancer were retrospectively included. Data concerning age; sex; diagnostic work-up; surgical procedures; and pathology reporting of tumor type, grade, size, receptor status and lymph nodes were retrospectively collected.

\section{Clinical nodal status}

Clinical nodal status was based on results of physical examination and axillary ultrasound, and classified according to the number of suspicious lymph nodes imaged by ultrasound, that is, cN1 is $1-3$, cN2 is $4-9$ and cN3 is $>9$ suspicious axillary lymph nodes. Tissue sampling was generally performed in axillae suspicious for malignancy on ultrasound using 16-18 gauge core needle biopsy. When core needle biopsy was technically challenging, for example due to the proximity of a blood vessel, fine needle aspiration cytology was used. In cases of multiple suspicious nodes, only the most suspicious node was sampled, and the number of suspicious nodes reported. The total number of suspicious and non-suspicious nodes was not reported. The axillary ultrasound was performed in every patient by dedicated breast radiologists (regardless of the results of physical examination) using an ATL-HDI5000 system, which in 2011 was replaced by an iU22-xMATRIX ultrasound system in combination with a high-frequency linear array transducer (Philips Medical Systems, Best, the Netherlands). If the sampled tissue contained no tumor cells or the ultrasound was negative, an SLNB was performed; otherwise an ALND was performed directly.

\section{Surgical techniques}

The SLN was identified by using a triple technique consisting of lymphoscintigraphy (using $80 \mathrm{MBq}$ Technetium-99m nanocolloid injected peri-areolar), blue dye to detect lymphatic vessels (Bleu Patente V; Guerbet, Aulnay-sous-Bois, France), and gamma probes to detect radioactivity. After the SLNB, a palpation of the axilla was performed 
to identify and remove suspicious non-SLNs. After the detection of one or more (micro-)metastasis, a completion level I-II ALND was performed.

\section{Pathological technique}

Core biopsies were routinely processed after formalin fixation. Sections stained with haematoxylin and eosin (H\&E) were assessed and additional immunohistochemical analyses were performed. Fine needle aspiration cytology was routinely processed: airdried smears were stained with May-Grünwald-Giemsa and liquid-based thin-layer cell preparations were stained with Papanicolaou and used for immunocytochemistry. SLNs were sliced with a maximum thickness of $3 \mathrm{~mm}$ and embedded in paraffin after formalin fixation. Each paraffin block was step sectioned at 500-micrometer intervals at three levels and stained with haematoxylin and eosin. If no metastasis was detected with haematoxylin and eosin, immunohistochemical staining was performed with a mouse anti-human MNF116 antibody (Dako).

All ALND lymph nodes were embedded in paraffin after formalin fixation. Lymph nodes larger than $5 \mathrm{~mm}$ were sliced with a maximum thickness of $3 \mathrm{~mm}$. All slides were stained with haematoxylin and eosin. Each node was recorded as either benign or as an isolated tumor cell (ITC) (pNO(i+)) $(\leq 0.2 \mathrm{~mm})$, micrometastasis (pN1mi) $(0.2 \leq 2.0 \mathrm{~mm})$ or macrometastasis $(\mathrm{pN}+)(>2.0 \mathrm{~mm})$.

\section{Statistical analyses}

Statistical analyses were performed using the Statistical Package for the Social Sciences (Version 19.0, Chicago, Illinois, USA). The false negative percentages and negative predictive values (NPVs) for axillary nodal staging with ultrasound were calculated for each nodal status in general and for CT1-2 patients treated by breast conserving therapy (BCT) in particular. Data were summarized as means with standard deviations (SD). Chi-square tests were used to analyze categorical data and independent-samples t-tests were used to analyze continuous data. $P$-value $<0.05$ was considered statistically significant.

\section{Results}

\section{Patient demographics and tumor characteristics}

During the study period, 647 patients were diagnosed with primary invasive breast cancer, 18 of whom had bilateral breast cancer, resulting in 665 possibly affected axillae. Clinical nodal status was based on axillary ultrasound ( \pm tissue sampling), and showed 561 axillae with cN0 (84.4\%), 85 with cN1 (12.8\%), and 19 with cN2 (2.8\%). No axillae were staged as $\mathrm{cN3}$. Axillae treated with neoadjuvant systemic therapy $(n=83)$ 
were excluded from further analysis, since clinical nodal status could not be directly compared with histopathology. Of the remaining 564 patients, 13 were diagnosed with bilateral breast cancer, resulting in 577 axillae eligible for analysis. Patient and tumor characteristics $(n=577)$ are summarized in Table 2.1. The mean age was 60 years (SD 12, range $25-88$ ) and T1 tumors were most frequently observed (65.7\%). Invasive ductal carcinoma (IDC) was the most common type of breast cancer in $79.9 \%$, followed by invasive lobular carcinoma (ILC) in $12.1 \%$, and other types of breast cancer in $8.0 \%$. In total, 278 cases were treated by BCT (48.2\%) and 299 by mastectomy $(51.8 \%)$.

The final histopathology reports showed 347 axillae without metastatic disease $(60.2 \%)$ and 230 axillae with metastatic disease (39.8\%). Macrometastases were identified in 138 (23.9\%), micrometastases in 37 (6.4\%), and ITCs in 55 axillae (9.5\%).

TABLE 2.1 Patient demographics and tumor characteristics for all patients and subdivided according to type of breast surgery (mastectomy or breast conserving therapy).

\begin{tabular}{|c|c|c|c|}
\hline Characteristic & Mastectomy & Breast conserving therapy & Total \\
\hline Number of axillae evaluated, N (\%) & $299(51.8)$ & $278(48.2)$ & 577 \\
\hline \multicolumn{4}{|l|}{ Age } \\
\hline Mean (SD) & $59.7(13.7)$ & $60.2(10.1)$ & $60(12)$ \\
\hline Range & $25-88$ & $30-87$ & $25-88$ \\
\hline Mean tumor size, mm (SD) & $25.1(18.4)$ & $14.8(7.3)$ & $20(15)$ \\
\hline \multicolumn{4}{|l|}{ T-stadium, N (\%) } \\
\hline $\mathrm{T} 1$ & $150(50.2)$ & $229(82.4)$ & $379(65.7)$ \\
\hline $\mathrm{T} 2$ & $117(39.1)$ & 49 (17.6) & $166(28.8)$ \\
\hline T3 & $29(9.7)$ & - & $29(5.0)$ \\
\hline T4 & $3(1.0)$ & - & $3(0.5)$ \\
\hline \multicolumn{4}{|l|}{ Tumor type, N (\%) } \\
\hline Ductal & 230 (76.9) & $231(83.1)$ & 461 (79.9) \\
\hline Lobular & $42(14.0)$ & $28(10.1)$ & $70(12.1)$ \\
\hline Ductolobular & $6(2.0)$ & $2(0.7)$ & $8(1.4)$ \\
\hline Mucinous & $5(1.7)$ & $6(2.2)$ & $11(1.9)$ \\
\hline Medullary & $2(0.7)$ & $3(1.1)$ & $5(0.9)$ \\
\hline Tubular & $2(0.7)$ & $2(0.7)$ & $4(0.7)$ \\
\hline Papillary & $3(1.0)$ & $2(0.7)$ & $5(0.9)$ \\
\hline Other & $9(3.0)$ & $4(1.4)$ & $13(2.2)$ \\
\hline \multicolumn{4}{|l|}{ Grade, N (\%) } \\
\hline 1 & $44(14.7)$ & $74(26.6)$ & $118(20.5)$ \\
\hline 2 & $142(47.5)$ & $123(44.2)$ & 265 (45.9) \\
\hline 3 & $113(37.8)$ & $81(29.1)$ & $194(33.6)$ \\
\hline \multicolumn{4}{|l|}{ Uni-/multifocal tumors, N (\%) } \\
\hline Unifocal & $220(73.6)$ & $267(96.0)$ & $487(84.4)$ \\
\hline Multifocal and/or centric & $79(26.4)$ & $11(4.0)$ & $90(15.6)$ \\
\hline \multicolumn{4}{|l|}{ Receptor status, N (\%) } \\
\hline Triple negative & $34(11.4)$ & $30(10.8)$ & $64(11.1)$ \\
\hline ER/PR + HER2/neu - & $211(70.6)$ & $217(78.1)$ & $428(74.2)$ \\
\hline ER/PR - HER2/neu + & $24(8.0)$ & $7(2.5)$ & $31(5.4)$ \\
\hline $\mathrm{ER} / \mathrm{PR}+\mathrm{HER} 2 /$ neu + & $29(9.7)$ & $23(8.3)$ & $52(9.0)$ \\
\hline Unknown & $1(0.3)$ & $1(0.4)$ & $2(0.3)$ \\
\hline
\end{tabular}

ER indicates Estrogen Receptor; PR: progesterone receptor; HER2/neu: human epidermal growth factor receptor 2 . 


\section{Results of physical examination and axillary ultrasound findings}

Lymph nodes were palpable by physical examination in 21 of the 577 axillae. Additionally, an axillary ultrasound ( \pm tissue sampling) was performed in all cases and resulted in cN0 in 522 (90.5\%), cN1 in 53 (9.2\%) and cN2 in two axillae (0.3\%). No axillae were staged as cN3. Table 2.2 shows the clinical nodal status with the corresponding postoperative nodal status, subdivided by results of physical examination of the axilla (no palpable nodes or palpable nodes) and type of breast surgery (breast conserving therapy or mastectomy).

TABLE 2.2 Clinical nodal status with corresponding postoperative nodal status of 577 axillae, subdivided according to axillary physical examination and type of breast surgery (breast conserving therapy or mastectomy).

\begin{tabular}{|c|c|c|c|c|c|c|c|c|}
\hline & & pNO & pNOi+ & pN1mi & pN1 & pN2 & pN3 & Total \\
\hline \multirow{6}{*}{ 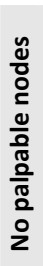 } & $\begin{array}{l}\text { Breast conserving therapy } \\
\text { cNO }\end{array}$ & 186 & 34 & 16 & 22 & 6 & - & 264 \\
\hline & $\mathrm{cN} 1$ & - & - & - & 3 & 2 & 3 & 8 \\
\hline & $\mathrm{cN} 2$ & - & - & - & - & - & - & 0 \\
\hline & $\begin{array}{l}\text { Mastectomy } \\
\text { cNO }\end{array}$ & 158 & 20 & 21 & 36 & 10 & 6 & 251 \\
\hline & cN1 & - & - & - & 22 & 6 & 4 & 32 \\
\hline & $\mathrm{cN} 2$ & - & - & - & - & 1 & - & 1 \\
\hline \multirow{8}{*}{$\begin{array}{l}\frac{y}{0} \\
\frac{0}{0} \\
\frac{0}{0} \\
\frac{0}{\pi} \\
\frac{\pi}{0}\end{array}$} & Breast conserving therapy & & & & & & & \\
\hline & $\mathrm{cNO}$ & 2 & - & - & - & - & - & 2 \\
\hline & cN1 & 0 & - & - & 3 & - & 1 & 4 \\
\hline & $\mathrm{cN} 2$ & - & - & - & - & - & - & 0 \\
\hline & Mastectomy & & & & & & & \\
\hline & cNO & 1 & 1 & - & 2 & - & 1 & 5 \\
\hline & cN1 & - & - & - & 3 & 2 & 4 & 9 \\
\hline & $\mathrm{cN} 2$ & - & - & - & - & - & 1 & 1 \\
\hline \multirow{4}{*}{$\begin{array}{l}\bar{\pi} \\
\stackrel{0}{0} \\
\qquad\end{array}$} & cNO & 347 & 55 & 37 & 60 & 16 & 7 & 522 \\
\hline & cN1 & - & - & - & 31 & 10 & 12 & 53 \\
\hline & cN2 & - & - & - & - & 1 & 1 & 2 \\
\hline & Total & 347 & 55 & 37 & 91 & 27 & 20 & 577 \\
\hline
\end{tabular}

cN1 is 1-3 and cN2 is 4-9 suspicious axillary lymph nodes imaged by ultrasound, of which at least one lymph node is a pathology-proven (biopsy / cytology) metastasis.

\section{Accuracy of negative axillary sonographic findings}

Of the axillae preoperatively staged as cNO $(n=522), 347$ were true negatives $(66.5 \%)$; 55 were diagnosed with ITCs (10.5\%); 37 with micrometastases (7.1\%); 60 with pN1 (11.5\%); 16 with pN2 (3.1\%); and seven with pN3 disease (1.3\%). Thus, advanced nodal disease (pN2-pN3) was present in 23 of 522 axillae with negative ultrasound findings, resulting in a false negative percentage of $4.4 \%(95 \% \mathrm{Cl} 2.9-6.6 \%)$ and an NPV of $95.5 \%$ (95\% Cl 93.4-97.1\%) for a negative axillary ultrasound to exclude advanced nodal disease. Advanced nodal disease in these 23 axillae arose from pT1-2 (73.9\%) and pT3-4 tumors (26.1\%) (Table 2.3). IDC breast cancer was found in 18 cases $(78.3 \%)$ and 
ILC breast cancer in five cases $(21.7 \%)$. There was no significant difference between the false negative percentage for advanced nodal disease arising from IDC and ILC: $4.3 \%$ versus $7.0 \%$, respectively $(P>0.05)$. The corresponding NPVs for IDC and ILC were $95.7 \%$ and $93.0 \%$, respectively $(P>0.05)$.

TABLE 2.3 Clinical nodal status with corresponding postoperative nodal status of 577 axillae, subdivided according to the postoperative primary tumor status.

\begin{tabular}{|c|c|c|c|c|c|c|c|c|}
\hline & \multicolumn{6}{|c|}{ Postoperative nodal status } & \multirow[b]{2}{*}{ TOTAL } \\
\hline & & pNO & pNOi+ & pN1mi & pN1 & pN2 & pN3 & \\
\hline \multirow{15}{*}{ 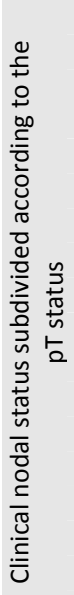 } & $\mathrm{cNO}$ & & & & & & & \\
\hline & рт1 & 269 & 38 & 22 & 30 & 5 & 1 & 365 \\
\hline & pT2 & 74 & 13 & 14 & 23 & 8 & 3 & 135 \\
\hline & рT3 & 3 & 4 & 1 & 6 & 2 & 3 & 19 \\
\hline & pT4 & 1 & - & - & 1 & 1 & - & 3 \\
\hline & cN1 ${ }^{\text {Total }}$ & 347 & 55 & 37 & 60 & 16 & 7 & 522 \\
\hline & pT1 & - & - & - & 9 & 1 & 4 & 14 \\
\hline & pT2 & - & - & - & 18 & 7 & 5 & 30 \\
\hline & рT3 & - & - & - & 4 & 2 & 3 & 9 \\
\hline & рT4 & - & - & - & - & - & - & 0 \\
\hline & $\mathrm{cN} 2^{\text {Total }}$ & 0 & 0 & 0 & 31 & 10 & 12 & 53 \\
\hline & рT1 & - & - & - & - & - & - & 0 \\
\hline & pт2 & - & - & - & - & - & 1 & 1 \\
\hline & рT3 & - & - & - & - & 1 & - & 1 \\
\hline & рT4 & - & - & - & - & - & - & 0 \\
\hline & Total & 0 & 0 & 0 & 0 & 1 & 1 & 2 \\
\hline TOTA & & 347 & 55 & 37 & 91 & 27 & 20 & 577 \\
\hline
\end{tabular}

cN1 is 1-3 and cN2 is 4-9 suspicious axillary lymph nodes imaged by ultrasound, of which at least one lymph node is a pathology-proven (biopsy / cytology) metastasis.

\section{Accuracy of positive axillary sonographic findings}

In the axillae preoperatively staged as $\mathrm{CN1}(\mathrm{n}=53)$, the ALND specimen contained pN1 disease in 31 (58.5\%), pN2 disease in 10 (18.9\%) and pN3 disease in 12 cases (22.6\%). Thus, advanced nodal disease (pN2-pN3) was present in 22 of 53 axillae staged as cN1, resulting in a false negative percentage of $41.5 \%(95 \% \mathrm{Cl} 28.4-55.8 \%)$ and an NPV of $58.5 \%(95 \% \mathrm{Cl} 44.2-71.5 \%)$ to exclude advanced nodal disease if a cN1 status is predicted by the axillary ultrasound. In the axillae preoperatively staged as cN2 $(n=2)$, the ALND specimen revealed one case of pN2 disease and one of pN3. The sample size was too small for further adequate statistical analysis.

\section{Nodal tumor load in patients with pN1 nodal status}

Axillae preoperatively staged as cN0, but with a postoperative pN1 status contained a median of 1.4 metastatic lymph nodes, versus 2.0 metastatic lymph nodes for axillae 
preoperatively staged as $\mathrm{CN} 1$ with a postoperative pN1 $(P<0.01)$. The differences between both groups in patient and tumor characteristics were not statistically significant (Table 2.4).

TABLE 2.4 Patient and tumor characteristics of $\mathrm{cNO}$ and $\mathrm{cN} 1$ patients with a postoperative pN1 status.

\begin{tabular}{|c|c|c|c|}
\hline & $\mathrm{cNO} \rightarrow \mathrm{pN} 1$ & $\mathrm{cN} 1 \rightarrow \mathrm{pN} 1$ & $P$-value \\
\hline Number of axillae & 60 & 31 & \\
\hline Mean age in years (SD) & $60(13.5)$ & $62(14.6)$ & 0.52 \\
\hline Mean tumor size in $\mathrm{mm}(\mathrm{SD})$ & $25.8(14.4)$ & $32.2(17.6)$ & 0.08 \\
\hline \multicolumn{4}{|l|}{ Primary tumor, $\mathrm{N}(\%)$} \\
\hline $\mathrm{T} 1$ & $30(50.0)$ & $9(29.0)$ & 0.06 \\
\hline $\mathrm{T} 2$ & $23(38.3)$ & $18(58.1)$ & 0.07 \\
\hline T3 & $6(10.0)$ & 4 (12.9) & 0.46 \\
\hline T4 & $1(1.7)$ & - & 0.66 \\
\hline \multicolumn{4}{|l|}{ Tumor type, N (\%) } \\
\hline Invasive ductal carcinoma & $48(80.0)$ & $28(90.3)$ & 0.21 \\
\hline Invasive lobular carcinoma & $12(20.0)$ & $2(6.5)$ & 0.09 \\
\hline Other & - & $1(3.2)$ & 0.34 \\
\hline \multicolumn{4}{|l|}{ Multi-focal/centric, N (\%) } \\
\hline Yes & $12(20.0)$ & $6(19.4)$ & 0.942 \\
\hline \multicolumn{4}{|l|}{ Receptor status, N (\%) } \\
\hline Triple negative & $6(10.0)$ & 4 (12.9) & 0.68 \\
\hline ER/PR + Her2/neu - & $41(68.3)$ & $21(67.7)$ & 0.95 \\
\hline ER/PR - Her2/neu + & $2(3.3)$ & $3(9.7)$ & 0.28 \\
\hline $\mathrm{ER} / \mathrm{PR}+\mathrm{Her} 2 /$ neu + & $11(18.3)$ & $3(9.7)$ & 0.22 \\
\hline \multicolumn{4}{|l|}{ SLNB } \\
\hline Mean number of SLN's removed (SD) & $2.2(1.2)$ & - & \\
\hline Total number of positive SLN's & 66 & - & \\
\hline Micrometastases & 6 & - & \\
\hline Macrometastases & 60 & - & \\
\hline Mean number of positive SLN's per axilla (SD) & $1.1(0.36)$ & - & \\
\hline \multicolumn{4}{|l|}{ (c)ALND } \\
\hline Mean number of LN's in (c)ALND (SD) & $15.5(6.1)$ & $18.3(6.6)$ & \\
\hline Number of axillae without additional metastases & 49 & - & \\
\hline Number of axillae with one additional metastasis & 8 & - & \\
\hline Number of axillae with two additional metastasis & 3 & - & \\
\hline Total number of positive LN's in (c)ALND & 14 & 59 & \\
\hline Micrometastases & 2 & 0 & \\
\hline Macrometastases & 12 & 59 & \\
\hline \multicolumn{4}{|l|}{ SLNB + (c)ALND } \\
\hline Mean number of positive LN's per axilla (SD) & $1.4(0.6)$ & $2.0(0.7)$ & $<0.01$ \\
\hline
\end{tabular}

ER indicates estrogen receptor; PR: progesterone receptor; HER2/neu: human epidermal growth factor receptor 2 SLNB, sentinel lymph node biopsy; (c)ALND: (completion) axillary lymph node dissection; LN: lymph node; SLN: sentinel lymph node.

\section{Axillary ultrasound findings for T1-2 patients treated by BCT}

Of the 278 cases treated by BCT, $82.4 \%$ had a pT1 tumor and $17.6 \%$ a pT2 tumor. The overall mean size was $14.8 \mathrm{~mm}$ (SD $7.3 \mathrm{~mm}$ ). In this subgroup, axillary ultrasound 
revealed no suspicious lymph nodes in 266 axillae (95.7\%). The remaining 12 axillae were all staged as cN1 (4.3\%).

In the 266 axillae preoperatively staged as cN0, 188 were true negatives (70.7\%); 34 were diagnosed with ITCs (12.8\%); 16 with micrometastases (6.0\%); 22 with pN1 (8.3\%); and 6 with pN2 disease (2.3\%). Thus, advanced nodal disease (pN2-pN3) was present in 6 of 266 axillae with negative ultrasound findings (cNO), resulting in a false negative percentage of $2.3 \%(95 \% \mathrm{Cl} 0.9-5.1 \%)$ and an NPV of $97.7 \%(95 \% \mathrm{Cl}$ 94.9-99.0\%) for a negative axillary ultrasound to exclude advanced nodal disease in this subgroup of patients.

In the 12 axillae preoperatively staged as cN1, the ALND specimen contained pN1 disease in six axillae (50.0\%), pN2 disease in two axillae (16.7\%) and pN3 disease in 4 axillae (33.3\%). Thus, advanced nodal disease was present in 6 of 12 axillae staged as cN1, resulting in a false negative percentage of $50.0 \%$ (95\% $\mathrm{Cl} 22.3-77.7 \%)$ and an NPV of $50.0 \%$ (95\% Cl $22.3-77.9 \%)$ to exclude advanced nodal disease if a cN1 status is predicted by the axillary ultrasound in this subgroup of patients. Of the six axillae with pN1 disease, three contained one and three contained two macrometastases. The two axillae with pN2 disease contained four and five macrometastases, respectively. The four axillae with pN3 disease contained 11, 13, 16 and 22 macrometastases, respectively. Three out of six axillae with pN1 disease had a clinically palpable lymph node, while only one of the six pN2-pN3 axillae had a clinically palpable lymph node.

\section{Discussion}

This study showed that a negative axillary ultrasound can exclude advanced nodal disease in almost all patients in a general breast cancer population, with an NPV of 95.5\%. In contrast, after a positive axillary ultrasound, it is impossible to differentiate between pN1 and pN2-3 disease. Furthermore, this study suggests that a negative axillary ultrasound selects pN1 patients with a lower tumor load, and therefore the more favorable pN1 disease. It could also be suggested that the CT1-2 patients treated by BCT in this study may have been overtreated with direct ALND after a positive axillary ultrasound, since the ALND specimen in 6 out of 12 axillae in this study contained only one or two macrometastases.

\section{General performance axillary ultrasound}

A meta-analysis by Houssami et al. showed that the sensitivity of axillary ultrasound with biopsy is $79.6 \%(95 \% \mathrm{Cl} 74.1-84.2 \%)$, with a specificity of $98.3 \%(95 \% \mathrm{Cl}$ $97.2-99.0 \%)^{6}$. Further, it has been reported by Stachs et al. that the accuracy of the axillary ultrasound depends mainly on the size of the lymph node metastases ${ }^{14}$. This is 
in line with the results of our study, because no patients with isolated tumor cells or micrometastases were identified by the axillary ultrasound.

Other publications on the accuracy of a negative axillary ultrasound for excluding advanced nodal disease report NPVs of $93.0-96.3 \%{ }^{15,16}$, compared to $95.5 \%$ in this study. Neil et al. mentioned significantly differing NPVs for IDC and ILC breast cancer (96 and $83 \%$ ) in contrast to this study $(96 \text { and } 93 \%)^{15}$. This conflicting finding may be caused by a lower number of patients with ILC breast cancer in the publication by Neal et al. compared to this study (47 vs. 78 patients). Choi et al. showed no significant difference between the NPVs for IDC compared to ILC breast cancer, which is similar to our current findings ${ }^{16}$.

To the best of our knowledge, there are no publications that report the accuracy of axillary ultrasound to exclude advanced nodal disease if a cN1 status is predicted, thus we are not able to compare our disappointing NPV of $58.8 \%$. However, this value is likely to be reliable since preoperative differentiation between cN1 and cN2 has always been important at our institution. According to the Dutch breast cancer guideline, adjuvant radiotherapy is standardly indicated after mastectomy in patients with pN2pN3 in contrast to pN1 patients ${ }^{5}$. It was policy in our institution to not perform an immediate breast reconstruction after mastectomy in patients for whom adjuvant radiotherapy is indicated, because patients who received postoperative radiotherapy after an immediate breast reconstruction were shown to be less satisfied and to have poorer cosmetic results ${ }^{17}$. Recent studies, however, reported satisfactory outcomes and no increased incidence of radiotherapy-associated complications ${ }^{18}$. Therefore, post mastectomy radiotherapy is no longer a contraindication for the performance of an immediate breast reconstruction in these patients.

\section{Consequences of performing standard preoperative axillary ultrasound in patients treated by mastectomy}

In the After Mapping of the Axilla: Radiotherapy Or Surgery? (AMAROS) trial, clinically node negative breast cancer patients (based on physical examination only) with a positive sentinel lymph node were randomized to undergo a complete ALND or radiotherapy to the axilla and periclavicular region. After a median follow-up of 6.1 years, regional control and overall survival were similar in both study arms, while the risk of lymphedema was reduced in the radiotherapy $\operatorname{arm}^{12}$. Only $18 \%$ of the patients included in this study were treated by mastectomy. It is therefore to discuss if results are applicable to all SLN-positive patients treated by mastectomy. Results of the AMAROS trial can be substantiated by results of the National Surgical Adjuvant Breast and Bowel Project (NSABP) B-04 trial, because this trial revealed that regional radiotherapy provides equally overall survival and regional control compared to the ALND, in clinically node negative breast cancer patients (again, solely physical examination, but without the SLNB) treated by mastectomy ${ }^{19}$. However, if clinicians are not confident to replace the completion ALND by radiotherapy in every SLN-positive 
patients treated by mastectomy, they could use the axillary ultrasound to select patients with favorable nodal tumor load, since our study showed that a negative axillary ultrasound excludes advanced nodal disease in $95.5 \%$ of the patients. On the contrary, the addition of the axillary ultrasound may result in that some patients miss the opportunity for a more axilla-conserving treatment according the AMAROS design.

\section{Consequences of performing standard preoperative axillary ultrasound in patients treated by breast conserving therapy}

The International Breast Cancer Study Group (IBCSG) 23-01 trial showed that a completion ALND can be safely omitted in breast cancer patients with micrometastatic disease in their $\operatorname{SLN}^{6}$. Patients in this trial were clinically node negative by physical examination and mainly treated by BCT (91\%). The axillary ultrasound in this subgroup of patients is not of added value, because our study showed that micrometastases are not detectable by ultrasound.

The ACOSOG Z0011 trial showed that omitting the completion ALND in SLN-positive breast cancer patients treated by BCT (i.e.: lumpectomy followed by tangential breast radiotherapy) and adjuvant systemic therapy, provides excellent overall survival and regional control ${ }^{13}$. Up until now, many breast clinics have not implemented the results in daily practice, because of criticism that followed after publication of the results ${ }^{20}$. Clinically node negative patients in the trial were selected by physical examination. Again, the addition of the axillary ultrasound may result in more confidence in omitting the completion ALND after a positive SLNB, since a negative axillary ultrasound excludes advanced nodal disease in $97.7 \%$ of the patients in this subgroup. On the contrary, the use of the axillary ultrasound will result in a decrease of the number of clinically node negative patients and subsequently an increase of the number of clinically node positive patients, and will thereby direct some patients to an ALND instead of an SLNB, which may result in overtreatment. Our study showed that according to the ACOSOG Z0011 design at least six of the 12 patients with a cN1 nodal status would miss the opportunity for a more axillary-conserving treatment, while their ALND specimen contained only 1-2 macrometastases. Furthermore, 278 axillary ultrasounds would need to be performed in this subgroup of patients to prevent an additional operation (completion ALND after positive SLNB) in the six patients (2.2\%) who were diagnosed with pN2 or pN3 disease in this study. Although this was the original purpose of implementing axillary ultrasound in the nodal work-up, this may not to be the most cost-effective strategy for this subgroup of patients ${ }^{21}$. However, it does seem justified to perform a new cost-effectively study to analyze this approach in current daily practice.

With these results in mind, it seems unjustified to perform standard axillary ultrasounds in the subgroup of patients with cT1-2 breast cancer and no palpable lymph nodes, who are treated by lumpectomy followed by tangential breast radiotherapy and adjuvant systemic therapy. 


\section{Future perspectives}

It may be possible in the future to omit the SLNB in patients with a negative axillary ultrasound. The axillary ultrasound can identify patients with no or limited axillary tumor load. To establish the safety of such an approach, different randomized controlled trials are conducted in patients treated by $\mathrm{BCT}^{22,23}$.

\section{Limitations}

Firstly, the retrospective design of this study may have led to a selection bias. However, our study population is comparable with the general breast cancer population in the Netherlands: $65.7 \%$ of the patients in our study had a T1 tumor compared to $59 \%$ in the Dutch breast cancer population ${ }^{24}$. The slight trend towards lower tumor stage could be the result of the neoadjuvant-treated patients being excluded. This could also be explained by the large number of tumors detected by screening high-risk family members ${ }^{25}$. Secondly, our study only sampled (biopsy/cytology) one node in cases of multiple suspicious nodes, which in theory may have led to a clinically overestimation of the nodal status. However, the results show that none of the patients had a higher clinical nodal status compared to their postoperative nodal status. Thirdly, excluding the neoadjuvant-treated patients may have led to an underestimated NPV of the negative axillary ultrasound to exclude advanced nodal disease, since neoadjuvanttreated patients mostly have larger tumors with a higher a priori chance of advanced nodal disease. The NPVs in our study show that these would likely have been detected by axillary ultrasound.

\section{Conclusion}

In conclusion, a negative axillary ultrasound generally excludes the presence of pN2pN3 disease. An axillary ultrasound cannot accurately differentiate between pN1 and pN2-pN3. It could be argued that the standard performance of an axillary ultrasound in breast cancer patients is questionable; multidisciplinary discussion could guide decisions on the use of axillary ultrasound for the individual patient. 


\section{References}

1. Krag DN, Anderson SJ, Julian TB, Brown AM, Harlow SP, Costantino JP, et al. Sentinel-lymph-node resection compared with conventional axillary-lymph-node dissection in clinically node-negative patients with breast cancer: overall survival findings from the NSABP B-32 randomised phase 3 trial. Lancet Oncol 2010;11:927-33.

2. Disipio T, Rye $S$, Newman B, Hayes $S$. Incidence of unilateral arm lymphedema after breast cancer: a systematic review and meta-analysis. Lancet Oncol 2013;14:500-15.

3. Dabakuyo TS, Fraisse J, Causeret S, Gouy S, Padeano MM, Loustalot C, et al. A multicenter cohort study to compare quality of life in breast cancer patients according to sentinel lymph node biopsy or axillary lymph node dissection. Ann Oncol 2009;20:1352-61.

4. Siesling S, van Dijck JA, Visser O, Coebergh JW. Trends in incidence of and mortality from cancer in The Netherlands in the period 1989-1998. Eur J Cancer 2003;39:2521-30.

5. NABON. National Breast Cancer Guideline. In. The Netherlands: Oncoline; 2012.

6. Houssami N, Ciatto S, Turner RM, Cody HS, 3rd, Macaskill P. Preoperative ultrasound-guided needle biopsy of axillary nodes in invasive breast cancer: meta-analysis of its accuracy and utility in staging the axilla. Ann Surg 2011;254:243-51.

7. Bilimoria KY, Bentrem DJ, Hansen NM, Bethke KP, Rademaker AW, Ko CY, et al. Comparison of sentinel lymph node biopsy alone and completion axillary lymph node dissection for node-positive breast cancer. J Clin Oncol 2009;27:2946-53.

8. Fant JS, Grant MD, Knox SM, Livingston SA, Ridl K, Jones RC, et al. Preliminary outcome analysis in patients with breast cancer and a positive sentinel lymph node who declined axillary dissection. Ann Surg Oncol 2003;10:126-30.

9. Guenther JM, Hansen NM, DiFronzo LA, Giuliano AE, Collins JC, Grube BL, et al. Axillary dissection is not required for all patients with breast cancer and positive sentinel nodes. Arch Surg 2003;138: 52-6.

10. Hwang RF, Gonzalez-Angulo AM, Yi M, Buchholz TA, Meric-Bernstam F, Kuerer HM, et al. Low locoregional failure rates in selected breast cancer patients with tumor-positive sentinel lymph nodes who do not undergo completion axillary dissection. Cancer 2007;110:723-30.

11. Jeruss JS, Winchester DJ, Sener SF, Brinkmann EM, Bilimoria MM, Barrera E, Jr., et al. Axillary recurrence after sentinel node biopsy. Ann Surg Oncol 2005;12:34-40.

12. Rutgers EJ, Donker M, Straver ME, Meijnen P, Van De Velde CJH, Mansel RE, et al. Radiotherapy or surgery of the axilla after a positive sentinel node in breast cancer patients: Final analysis of the EORTC AMAROS trial (10981/22023). J Clin Oncol 2013;31(suppl; abstr LBA1001).

13. Giuliano AE, Hunt KK, Ballman KV, Beitsch PD, Whitworth PW, Blumencranz PW, et al. Axillary dissection vs no axillary dissection in women with invasive breast cancer and sentinel node metastasis: a randomized clinical trial. JAMA 2011;305:569-75.

14. Stachs A, Göde K, Hartmann S, Stengel B, Nierling U, Dieterich M, et al. Accuracy of axillary ultrasound in preoperative nodal staging of breast cancer - size of metastases as limiting factor. SpringerPlus 2013;2:350.

15. Neal CH, Daly CP, Nees AV, Helvie MA. Can preoperative axillary US help exclude N2 and N3 metastatic breast cancer? Radiology 2010;257:335-41.

16. Choi JS, Kim MJ, Moon HJ, Kim EK, Yoon JH. False negative results of preoperative axillary ultrasound in patients with invasive breast cancer: correlations with clinicopathologic findings. Ultrasound Med Biol 2012;38:1881-6.

17. Cordeiro PG, Pusic AL, Disa JJ, McCormick B, VanZee K. Irradiation after immediate tissue expander/implant breast reconstruction: outcomes, complications, aesthetic results, and satisfaction among 156 patients. Plast Reconstr Surg 2004;113:877-81.

18. Schaverien MV, Macmillan RD, McCulley SJ. Is immediate autologous breast reconstruction with postoperative radiotherapy good practice?: A systematic review of the literature. J Plast Reconstr Aesthetic Surg 2013;66:1637-51.

19. Fisher B, Jeong JH, Anderson S, Bryant J, Fisher ER, Wolmark N. Twenty-five-year follow-up of a randomized trial comparing radical mastectomy, total mastectomy, and total mastectomy followed by irradiation. N Engl Med 2002;347:567-75. 
20. Hasselle MD, Dorn PL. Axillary vs sentinel lymph node dissection for invasive breast cancer. JAMA 2011;305:2289; author reply 2290-1.

21. Turaga KK, Chau A, Eatrides JM, Kiluk JV, Khakpour N, Laronga C, et al. Selective application of routine preoperative axillary ultrasonography reduces costs for invasive breast cancers. Oncologist 2011;16:942-8.

22. Gentilini O, Veronesi U. Abandoning sentinel lymph node biopsy in early breast cancer? A new trial in progress at the European Institute of Oncology of Milan (SOUND: Sentinel node vs Observation after axillary UltraSouND). Breast 2012;21:678-81.

23. van Roozendaal LM, de Wilt JHW, Smidt ML. Clinical node negative breast cancer patients undergoing breast conserving therapy: follow-up versus sentinel lymph node biopsy. In: San Antonio Breast Cancer Symposium: Cancer Research 72:567s; 2012.

24. van der Heiden-van der Loo M, Ho VK, Damhuis RA, Siesling S, Menke MB, Peeters PH, et al. [Percentage of local recurrence following treatment for breast cancer is not a suitable performance indicator]. Ned Tijdschr Geneeskd 2010;154:A1984.

25. Tilanus-Linthorst MM, Bartels CC, Obdeijn Al, Oudkerk M. Earlier detection of breast cancer by surveillance of women at familial risk. Eur J Cancer 2000;36:514-9. 


\title{
Chapter
}

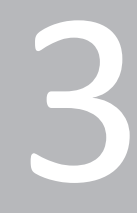

The diagnostic performance of dedicated axillary T2- and diffusion-weighted MR imaging for nodal staging in breast cancer

\author{
RJ. Schipper \\ M. Paiman \\ R.G.H. Beets-Tan \\ P.J. Nelemans \\ B. de Vries \\ E.M. Heuts \\ K.K. van de Vijver \\ K.B.M.I. Keymeulen \\ B.T. Brans \\ M.L. Smidt \\ M.B.I. Lobbes
}

Radiology 2015 May 275:345-55 


\section{Abstract}

Purpose

To evaluate the diagnostic performance of unenhanced axillary T2-weighted and diffusionweighted (DW) magnetic resonance imaging (MR imaging) for axillary nodal staging for patients with newly diagnosed breast cancer, with node-by-node and patient-by-patient validation.

\section{Materials and methods}

Institutional review board approval and informed consent were obtained. Fifty women (mean age, 60 years; range, 22-80 years) underwent high-resolution axillary 3.0-T MR imaging, T2-weighted imaging without fat suppression and DW MR imaging ( $b$ values 0,500 , $800 \mathrm{sec} / \mathrm{mm}^{2}$ ), followed by either sentinel lymph node biopsy (SLNB) or axillary lymph node dissection. Two radiologists independently scored each lymph node on a confidence level scale from 0 (benign) to 4 (malignant), first on T2-weighted MR images, then on DW MR images. Two researchers independently measured the mean apparent diffusion coefficient (ADC) of each lymph node. Diagnostic performance parameters were calculated on the basis of node-by-node and patient-by-patient validation.

Results

With respective node-by-node and patient-by-patient validation, T2-weighted MR imaging had a specificity of $93-97 \%$ and $87-95 \%$, sensitivity $32-55 \%$ and $50-67 \%$, negative predictive value (NPV) of $88-91 \%$ and $86-89 \%$, positive predictive value (PPV) of $60-70 \%$ and $62-75 \%$; area under the receiver operating characteristic curve (AUC) of 0.78 and $0.80-0.88$, with good interobserver agreement ( $\mathrm{K} 0.70)$. The addition of DW MR imaging resulted in lower specificity $(59-88 \%$ and $50-84 \%)$, higher sensitivity (45-64\% and 75-83\%), comparable NPV (89\% and 90-91\%), lower PPV (23-42\% and 34-60\%), and lower AUC (0.68-0.73 and 0.70-0.86). ADC measurement resulted in a specificity of $63-64 \%$ and $61-63 \%$, sensitivity of $41 \%$ and $67 \%$, NPV of $85 \%$ and $85-86 \%$, PPV of $18 \%$ and $35-36 \%$ and AUC of $0.54-0.58$ and $0.69-0.74$, respectively, with excellent interobserver agreement (interclass correlation coefficient 0.83 ).

\section{Conclusion}

Dedicated high-resolution axillary T2-weighted MR imaging showed good specificity on the basis of node-by-node and patient-by-patient validation, with good interobserver agreement. However, its NPV is still insufficient to substitute it for SLNB for exclusion of axillary lymph node metastasis. DW MR imaging and ADC measurement were of no added value. 


\section{Introduction}

The treatment regimen for axillary lymph node metastases in patients with breast cancer has changed during the last three years ${ }^{1,2}$. Until recently, a completion axillary lymph node dissection (ALND) was indicated after the detection of any axillary lymph node metastasis with axillary ultrasound guided biopsy or sentinel lymph node biopsy (SLNB). However, three randomized controlled trials showed that regional control or overall survival was not affected by omission of axillary treatment in the case of sentinel lymph node metastasis ${ }^{3-5}$. Also, the effect of the detection of a single axillary metastasis on adjuvant systemic treatment appears limited ${ }^{6}$. Consequently, the need for the detection of every single small lymph node (micro)metastasis has become questionable. This creates a window of opportunity for dedicated axillary imaging techniques that might have sufficient negative predictive value (NPV) to omit surgical staging of the axilla by using SLNB.

Although the SLNB has replaced routine ALND in most patients, it remains associated with morbidity, such as seroma, hematoma, lymphedema, numbness, tingling and pain $^{7}$. Lymphedema occurs in $8 \%$ of the patient after a follow-up of 3 years ${ }^{8}$. A noninvasive axillary staging method that could be substituted for SLNB and prevent its associated morbidity would therefore be important.

Current imaging modalities, including preoperative breast magnetic resonance (MR) imaging, positron-emission tomography (PET) or computer tomography (CT) are considered to be insufficient in terms of diagnostic performance to serve as an alternative for $\mathrm{SLNB}^{9-12}$. With respect to $\mathrm{MR}$ imaging, previous investigators mainly evaluated lymph nodes visible on (preoperative) breast MR imaging instead of using dedicated, high spatial resolution axillary MR imaging sequences ${ }^{13-17}$. It has been shown that the use of dedicated axillary MR imaging protocols improves the accuracy of MR imaging in nodal staging ${ }^{10,18-20}$. An important limitation of previous studies is the evaluation of the diagnostic accuracy solely on a patient-by-patient level and studies on a node-by-node level are still lacking.

We hypothesized that dedicated high spatial resolution axillary MR imaging, in combination with an imaging protocol consisting of small-voxel and thin-section T2-weighted images, can be used to acquire accurate information on axillary lymph node morphology. With the addition of information on diffusivity, which reflects microstructural changes, the diagnostic performance could be improved even further by adding diffusion-weighted (DW) imaging.

The aim of this prospective study was to evaluate the diagnostic performance of unenhanced axillary T2-weighted and DW MR imaging for axillary nodal staging in patients with newly diagnosed breast cancer with both node-by-node and patient-bypatient validation. 


\section{Materials and methods}

\section{Patients}

The local ethical committee approved the study and written informed consent was obtained. This study was designed as a prospective single-center cohort study. Consecutive patients with newly diagnosed histopathologically confirmed breast cancer, who were about to undergo nodal staging, were considered for inclusion in the period of August 2012 to December 2013. Exclusion criteria were neoadjuvant systemic therapy prior to lymph node surgery, age less than 18 years, pregnancy and general contra-indications for MR imaging.

\section{Imaging}

Axillary MR examinations were performed prior to axillary lymph node surgery on a 3.0-T MR imaging unit (Achieva 3.0TX; Philips Healthcare, Best, The Netherlands) by using a 32-channel cardiac sensitivity encoding coil with the patient in supine position and the ipsilateral arm elevated with close contact between coil and axilla. The imaging protocol consisted of the following: (a) three-dimensional T2-weighted turbo spin echo sequence without fat suppression (repetition time (TR) $2000 \mathrm{msec}$; echo time (TE) $153 \mathrm{msec}$; flip angle (FA) 90 degrees; turbo spin echo factor 50; number of signal acquired (NSA) 1; image coverage $93.75 \mathrm{~mm} ; 75$ coronal sections acquired; acquisition section thickness 2.50-mm; reconstruction section thickness $1.25-\mathrm{mm}$; field of view $220 \times 220 \mathrm{~mm}^{2}$; matrix 176x176; resulting in an in-plane resolution of $1.25 \times 1.25 \mathrm{~mm}^{2}$; total imaging time of 3 minutes 26 seconds; and (b) a coronal DW imaging sequence with fat suppression, $b$ values of 0,500 and $800 \mathrm{sec} / \mathrm{mm}^{2}$ per axis (TR $2108 \mathrm{msec}$; TE $52 \mathrm{msec}$; echo planar imaging factor 71; number of signals acquired 8; image coverage $95.4 \mathrm{~mm}$; 29 coronal sections; acquisition section thickness $3.00-\mathrm{mm}$; reconstruction section thickness $3.00-\mathrm{mm}$; actual section gap $0.30-\mathrm{mm}$; field of view $220 \times 220 \mathrm{~mm}^{2}$; matrix 160x160; in-plane resolution of voxel size $1.38 \times 1.38 \mathrm{~mm}^{2}$; and total imaging time, 5 minutes 42 seconds. Apparent diffusion coefficient (ADC) maps, in which the pixel "gray value" represents the calculated ADC for each voxel, were generated at the software console built into the MR unit by using all three $b$ values.

Prior to the SLNB, a single photon emission computed tomography-X-ray computed tomography (SPECT-CT) scan was performed, if feasible (Precedence 6-slice SPECT-CT scanner; Philips Healthcare, Best, The Netherlands). Intradermal injection of $80 \mathrm{MBq}$ of technetium-99m nanocolloid in the periareolar region of the ipsilateral breast was followed two hours later by lymphoscintigraphic images of the axilla and SPECT-CT with the patient in supine position and both arms positioned above the head. In our hospital the preoperative lymphoscintigraphic images are part of the routine triple technique used by the surgeon for identification of the sentinel lymph nodes (lymphoscintigraphy, 
blue dye (Bleu Patente V; Guerbet, Aulnay-sous-Bois, France) to detect lymphatic vessels and a gamma probe to detect radioactivity).

\section{Image analysis}

Two radiologists (R.BG.H.B.T., with 15 years of experience in pelvic MR imaging and lymph node assessment in rectal cancer [reader 1] and M.B.I.L., with 5 years of experience in breast MR imaging and in axillary lymph node assessment using ultrasound and 2 years of experience in axillary lymph node assessment on preoperative breast MR imaging [reader 2]) independently analyze T2-weighted and DW MR images and were blindedto histopathological findings. Similar to routine clinical practice, they were aware of the clinical tumor size, which was defined on preoperative breast ultrasound or, if available, on preoperative breast MR imaging.

On T2-weighted MR images, each node was scored by using a confidence level scale of 0 (definitely benign) to 4 (definitely malignant), by using the criteria as described by Baltzer et al. ${ }^{18}$. Characteristics included in the qualitative assessment of malignancy were irregular margins, inhomogeneous cortex, perifocal edema and absent fatty hilum Next, DW MR images (b-value $800 \mathrm{sec} / \mathrm{mm}^{2}$ ) and ADC maps (without the quantitative data presented as numbers, only as "gray values") were presented to the radiologists for qualitative analysis, as addition to the T2-weighted MR imaging images. Reader 1 assessed those strictly by using the criteria formulated by Scaranelo et al. ${ }^{13}$; each lymph node was again scored from 0 to 4 , independent of the initial score based on T2-weighted MR images. Reader 2 used the additional information provided by DW MR images and ADC maps to adjust his score on the basis of T2-weighted MR images.

The DW MR images were also assessed quantitatively. Two readers (M.L.P. [reader 3] and R.J.S. [reader 4]), both researchers, with a focus on dedicated axillary lymph node imaging, independently measured mean ADC of all visible lymph nodes, and were blinded to histopathological findings and image interpretation results of readers 1 and 2 (OsiriX 5.8.5, Pixmeo, Geneva, Switzerland). Each lymph node marked on T2-weighted MR image by a radiologist was identified on the corresponding DW image. A region of interest for each lymph node was manually drawn on DW images obtained with b-value $800 \mathrm{sec} / \mathrm{mm}^{2}$. In sub centimeter lymph nodes, the whole lymph node was included. In larger lymph nodes, only the cortex was delineated, since the outline of the fatty hilum is difficult to distinguish. Only the region with evidently high signal intensity compared with surrounding tissue was incorporated, to ensure that only lymph node parenchyma was included. The region of interest was copied to the ADC map, and the mean ADC value was calculated.

Assessment of SPECT-CT images for identification of the sentinel lymph nodes on axillary MR imaging was conducted by two researchers in consensus, who were blinded for pathological and T2-weighted and DW MR imaging results (M.L.P. and R.J.S.). 


\section{Histopathological analysis}

Lymph nodes were surgically removed at SLNB or ALND. ALND was performed by means of en bloc resection of axillary tissue. Immediately after dissection, landmarks were applied for anatomical reference (top/bottom of axilla, medial/lateral side). Two researchers (M.L.P. and R.J.S.) were responsible for fixation of the specimen in anatomical position

Lymph nodes were analyzed at pathologic examination according to guidelines published previously ${ }^{21}$. Sentinel lymph nodes were sliced with a maximum thickness of $3 \mathrm{~mm}$, step sectioned at three levels at 500-micrometer intervals and stained with hematoxylin and eosin. If no metastasis was detected, immunohistochemical staining was performed. Of the ALND specimens, lymph nodes with a diameter larger than $5 \mathrm{~mm}$ were sliced with a maximum thickness of $3 \mathrm{~mm}$. All slides were stained with hematoxylin and eosin.

Each node was recorded as either benign (pNO) or as isolated tumor cells (ITC) (pNO(i+)) $(\leq 0.2 \mathrm{~mm})$, micrometastasis $(\mathrm{pN} 1 \mathrm{mi})(0.2 \leq 2.0 \mathrm{~mm})$ or macrometastasis (pN1-3) $(>2.0 \mathrm{~mm})$. Surgeons and pathologists were blinded to the results of T2-weighted and DW imaging results.

\section{Evaluation of diagnostic performance}

The diagnostic performance of T2-weighted MR imaging and T2-weighted MR imaging with the addition of DW MR imaging (T2-weighted+DW MR imaging) was evaluated by means of (a) node-by-node analysis and (b) patient-by-patient analysis.

For the lymph nodes dissected at SLNB, the matching of histopathological findings with axillary MR imaging was conducted by using a preoperative SPECT-CT scan. The coronal SPECT-CT that showed the lymph node with high uptake of radioactive tracer was viewed and corresponding coronal slice of the T2-weighted MR imaging acquired at the same level in the axilla was retrieved (Figure 3.1).

For the lymph nodes harvested at ALND, both an anatomical map based on the ALND specimen, which was fixed in the anatomical situation immediately after surgery, and an anatomical map based on the T2-weighted MR image were drawn. Details on the node-by-node matching procedure were published earlier by Schipper et al. ${ }^{20}$. Node-bynode matching was achieved by means of side-by-side comparison of those two anatomical maps (Figure 3.2).

The matching of SPECT-CT images (for SLNB) and ALND specimens with axillary MR imaging was performed by two researchers (M.L.P. and R.J.S.) in consensus; both were blinded to the pathology findings and qualitative T2-weighted and DW results. 


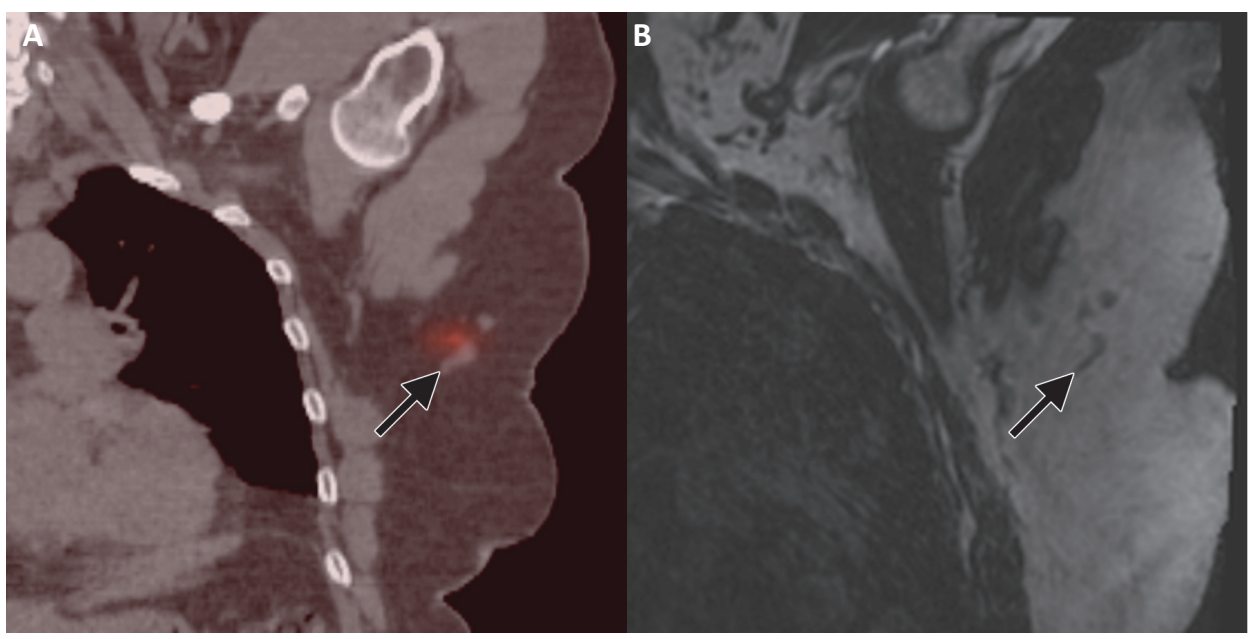

Figure 3.1 Images obtained in a 71-year old women with invasive ductal carcinoma in the left breast, which was treated with lumpectomy and the sentinel lymph node procedure (stage pt1N0). A: Coronal SPECT-CT image of the left axilla with arm elevated and B: corresponding axillary T2-weighted MR image of the left axilla (as shown to the radiologist for lymph node assessment) demonstrate the matching procedure for the sentinel lymph nodes (arrows). On the preoperative SPECT-CT image, the sentinel lymph node is highlighted. This enables identification of the sentinel lymph node on the corresponding MR image.

\section{Statistical analysis}

Statistical analyses were performed by using Statistical Package for the Social Sciences software (version 22.0; IBM SPSS Statistics for Windows. Armonk, NY) and R Project (version 3.0.1; R Foundation for Statistical Computing, Vienna, Austria). Diagnostic performance parameters, including sensitivity, specificity, positive predictive value (PPV), NPV and area under the receiver operating characteristic curve (AUC), were analyzed for T2-weighted MR imaging, T2-weighted with DW MR imaging, and quantitative $A D C$ measurements. At the start of the study, the cut-off at the confidence level scale was defined between 2 (possibly benign) and 3 (probably malignant), with lymph nodes scored 2 or lower categorized as benign and those scored 3 or higher as malignant.

For the node-by-node analysis, MR imaging findings for each node were compared with histopathologic findings. Isolated tumor cells and micrometastasis found at histopathologic analysis were neglected and recorded as negative. Since lymph nodes are clustered per patient when interpreting axillary MR imaging, individual lymph node observations cannot be regarded as independent. To adjust for this within-patient correlation, a bootstrap analysis was added (number of repetitions 1000 for calculation of the $95 \%$ confidence intervals (Cls) for the diagnostic performance parameters) ${ }^{22}$. 
A

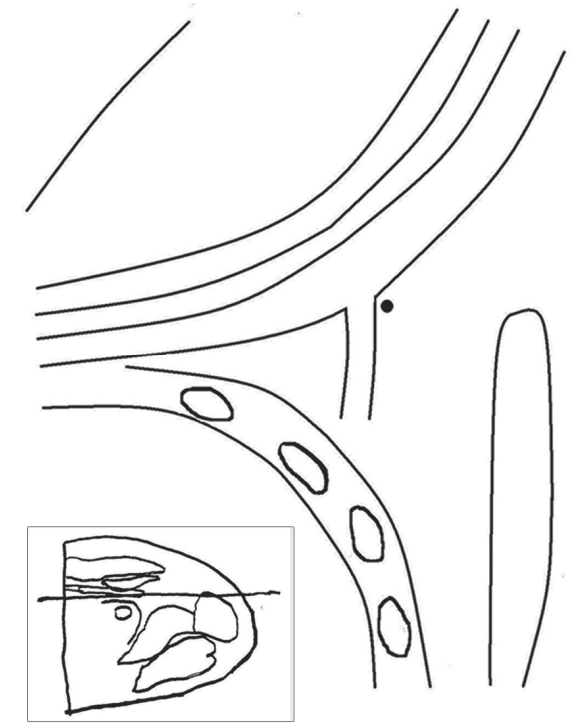

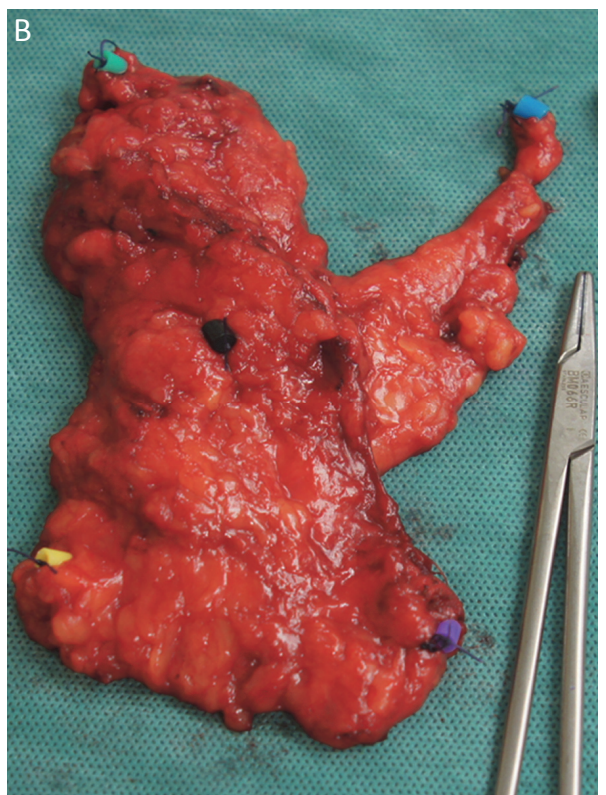

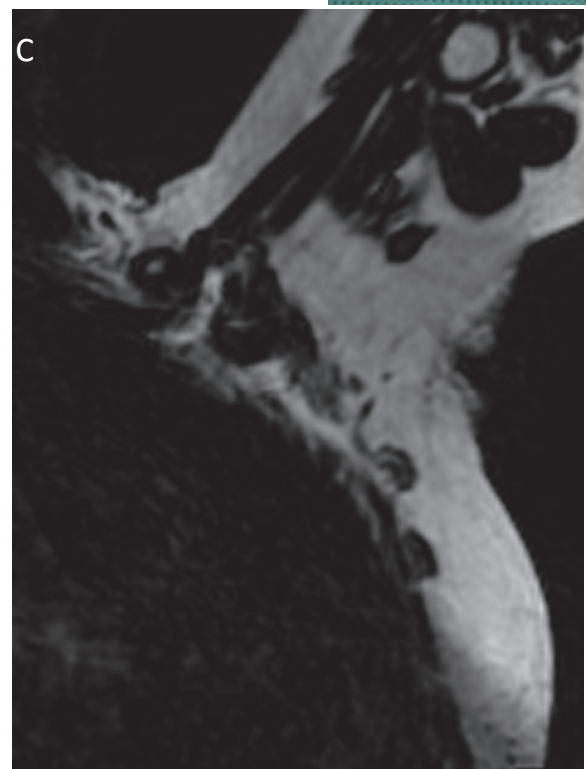

Figure 3.2 Matching procedure for lymph nodes harvested by axillary lymph node dissection, using anatomical maps. Images of a woman aged 50 years with an invasive ductal carcinoma in the left breast, treated with mastectomy and axillary lymph node dissection (pT2N2). A: Schematic anatomical map of the axilla (coronal plane) which was used to mark the anatomical position of the lymph nodes in the dissection specimen and the lymph nodes visible on axillary MR images. Node-by-node matching was done by side-by-side comparison of those two anatomical maps. B: Axillary lymph node dissection specimen (fixed in anatomical position). C: Axillary T2 weighted MR image (coronal plane). 
For the patient-by-patient analysis, in patients who underwent with SLNB alone, only the lymph nodes on axillary MR imaging identified as sentinel lymph nodes were taken into account. Regarding patients who underwent ALND, all lymph nodes depicted on axillary MR imaging were included into the patient-by-patient analysis.

The diagnostic performance of T2-weighted MR imaging and T2-weighted + DW MR imaging was compared by using the bootstrap test for the node-by-node validation and DeLong's test for the patient-by-patient validation ${ }^{23}$. The interobserver agreement of the qualitative image analysis was evaluated by the kappa statistic ( $\mathrm{k}$ ) and the interobserver agreement of the quantitative ADC analysis by using a Bland-Altman plot and the intraclass correlation coefficient (ICC). To compare the ADC values of histopathologically confirmed benign and malignant lymph nodes, a Wilcoxon rank sum test was performed. $P$-values less than 0.05 were considered to indicate a statistically significant difference. We performed a post hoc power calculation for AUC. The estimation of power was based on a two-sided test with 0.05 type I error rate.

\section{Results}

\section{Patients}

Fifty-four patients were included and underwent axillary MR imaging. One patient was excluded since no axillary lymph nodes were surgically removed. In one patient, histopathologic findings of the axillary lymph nodes showed chronic lymphocytic leukemia. Two patients were excluded owing to inadequate image quality of the DW images, based on motion artifacts. Consequently, 50 patients were included for final analysis. Patient and tumor characteristics are summarized in Table 3.1. The median time interval between axillary MR imaging and axillary surgery was 5 days (range 0-30 days).

\section{Histopathologic findings}

A total of 171 lymph nodes were removed during SLNB (87 lymph nodes in 45 patients) or ALND (84 lymph nodes in 5 patients), of which 135 were matched with lymph nodes identified on axillary T2-weighted MR imaging. The 36 harvested lymph nodes that did not match with MR imaging findings were all benign. Histopathologic examination of the 135 lymph nodes resulted in 22 (16\%) macrometastases), and 113 (84\%) negative findings (including three cases of ITC and ten cases micrometastases) (Table 3.2). In 12 of the 50 patients $(24 \%)$ one or more axillary macrometastases were found at histopathologic examination. 
Table 3.1 Patient demographics and tumor characteristics.

\begin{tabular}{|c|c|}
\hline Characteristic & Value \\
\hline Number of Patients & 50 \\
\hline \multicolumn{2}{|l|}{ Age, years } \\
\hline Mean (SD) & $60(12)$ \\
\hline Range & $22-80$ \\
\hline \multicolumn{2}{|l|}{ Clinical tumor size in situ carcinoma, $\mathrm{mm}$} \\
\hline Mean (SD) & $40(30)$ \\
\hline Range & $24-85$ \\
\hline \multicolumn{2}{|l|}{ Clinical tumor size invasive carcinoma, $\mathrm{mm}$} \\
\hline Mean $(S D)$ & $20(11)$ \\
\hline Range & $6-64$ \\
\hline \multicolumn{2}{|l|}{ cT-stage, n (\%) } \\
\hline Tis & $4(8)$ \\
\hline $\mathrm{T} 1$ & $27(54)$ \\
\hline $\mathrm{T} 2$ & $18(36)$ \\
\hline $\mathrm{T} 3$ & $1(2)$ \\
\hline \multicolumn{2}{|l|}{ pN-stage, n (\%) } \\
\hline $\mathrm{pNO}$ & $32(64)$ \\
\hline $\mathrm{pNO}(\mathrm{i}+)$ & $1(2)$ \\
\hline pN1mi & $5(10)$ \\
\hline pN1 & $9(18)$ \\
\hline $\mathrm{pN} 2$ & $3(6)$ \\
\hline \multicolumn{2}{|l|}{ Tumor type, n (\%) } \\
\hline Ductal carcinoma in situ & $4(8)$ \\
\hline Ductal & $35(70)$ \\
\hline Lobular & $8(16)$ \\
\hline Other & $3(6)$ \\
\hline \multicolumn{2}{|l|}{ Uni-/multifocal tumors, n (\%) } \\
\hline Unifocal & $39(78)$ \\
\hline Multifocal and/or multicentric & $11(22)$ \\
\hline \multicolumn{2}{|l|}{ Hormonal receptor and HER2 status, n (\%) } \\
\hline Triple negative & $4(8)$ \\
\hline$E R / P R+H E R 2-$ & $35(70)$ \\
\hline$E R / P R-H E R 2+$ & $2(4)$ \\
\hline $\mathrm{ER} / \mathrm{PR}+\mathrm{HER} 2+$ & $5(10)$ \\
\hline Not determined (in case of in situ carcinoma) & $4(8)$ \\
\hline
\end{tabular}

$\mathrm{N}$ : number of cases; SD: standard deviation; ER: estrogen receptor; PR: progesterone receptor; HER2: human epidermal growth factor receptor 2.

Table 3.2 Lymph node scoring on axillary MR imaging and findings by histopathology.

\begin{tabular}{|c|c|c|c|c|c|c|}
\hline & & & \multicolumn{4}{|c|}{ Histopathology } \\
\hline & & & Benign & Isolated tumor cells & Micrometastasis & Macrometastasis \\
\hline \multirow[t]{4}{*}{ R1 } & $\mathrm{T} 2 \mathrm{~W}$ & - & 93 & 3 & 9 & 10 \\
\hline & & + & 7 & 0 & 1 & 12 \\
\hline & $\mathrm{T} 2 \mathrm{~W}+\mathrm{DW}$ & - & 61 & 2 & 4 & 8 \\
\hline & & + & 39 & 1 & 6 & 14 \\
\hline \multirow[t]{4}{*}{ R2 } & $\mathrm{T} 2 \mathrm{~W}$ & - & 97 & 3 & 10 & 15 \\
\hline & & + & 3 & 0 & 0 & 7 \\
\hline & $\mathrm{T} 2 \mathrm{~W}+\mathrm{DW}$ & - & 87 & 3 & 9 & 12 \\
\hline & & + & 13 & 0 & 1 & 10 \\
\hline
\end{tabular}

$\mathrm{R} 1$ : radiologist 1; R2: radiologist 2; T2W: T2-weighted MR imaging; DW: diffusion-weighted MR imaging. Absolute numbers are presented. 


\section{Diagnostic performance and interobserver agreement}

On the basis of node-by-node validation, T2-weighted MR imaging had the following diagnostic performance of specificity $93-97 \%$; sensitivity $32-55 \%$; NPV of $88-91 \%$; PPV of $60-70 \%$, and AUC of 0.78 for reader 1 and 0.78 for reader 2), with moderate interobserver agreement $(\mathrm{k}=0.56)$. The addition of DW MR imaging resulted in lower specificity (59-88\%), higher sensitivity (45-64\%), comparable NPV (89\%), lower PPV (23-42\%), and lower AUC for reader $1(0.68 P=0.037)$ and comparable AUC for reader 2 (0.73, $P=0.368)$. Post hoc analysis showed that a sample size of $\mathrm{N}=135$ lymph nodes has a power of 0.68 to detect a 0.10 difference in AUC (null hypothesis: AUC for T2-weighted and DW MR imaging both 0.78).

Mean ADC values $(95 \% \mathrm{Cl})$ for benign nodes were $0.754(0.706-0.801)$ and 0.747 $(0.701-0.792) \times 10^{-3} \mathrm{~mm}^{2} / \mathrm{sec}$ and for malignant nodes $0.666(0.524-0.808)$ and 0.712 $(0.570-0.854) \times 10^{-3} \mathrm{~mm}^{2} / \mathrm{sec}$ for reader 3 and 4 , respectively. There was no significant difference between mean ADC values of benign and malignant nodes ( $P=0.219$ and 0.590 for reader 3 and 4 , respectively).

A cut-off of $0.650 \times 10^{-3} \mathrm{~mm}^{2} / \mathrm{sec}$ was chosen, since this provided good specificity with reasonable sensitivity. ADC measurement resulted in a specificity of $63-65 \%$, sensitivity of $41 \%$, NPV of $85 \%$, PPV of $18 \%$ and AUC of $0.54-0.58$, with excellent interobserver agreement (mean difference $-0.0016 \times 10^{-3} \mathrm{~mm}^{2} / \mathrm{sec}$; $95 \%$ limits of agreement $-0.30542 \times 10^{-3}, 0.30222 \times 10^{-3} \mathrm{~mm}^{2} / \mathrm{sec}$ and ICC $=0.83$ ). The corresponding Bland-Altman plot is presented in Figure 3.3.

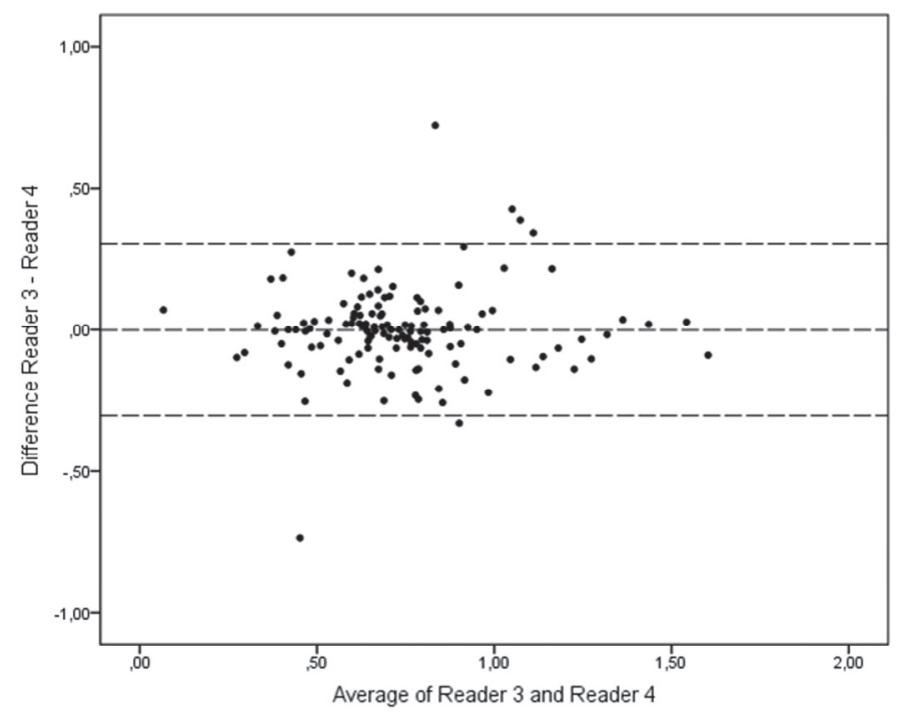

Figure 3.3 Bland-Altman plot for the difference in ADC measurement between reader 3 and 4, related to the average value for $A D C$ of reader 3 and 4 . Mean difference in measurement pairs was $-0.0016 \times 10^{-3} \mathrm{~mm}^{2} / \mathrm{sec} ; 95 \%$ limits of agreement $-0.30542 \times 10^{-3}, 0.30222 \times 10^{-3} \mathrm{~mm}^{2} / \mathrm{sec}$. 
For the patient-by-patient validation, T2-weighted MR imaging had the following diagnostic performance (specificity 87-95\%; sensitivity 50-67\%; NPV 86-89\%; PPV 62-75\%; AUC 0.80-0.88), with good agreement ( $\mathrm{k}=0.70)$. The addition of DW MR imaging resulted in lower specificity (50-84\%), higher sensitivity $(75-83 \%)$, comparable NPV (90-91\%), lower PPV (34-60\%) and lower AUC (0.70, $P=0.010)$ for reader 1 and comparable AUC (0.86, $P=0.524)$ for reader 2.

ADC measurement resulted in a specificity of $61-63 \%$, sensitivity of $67 \%$, NPV of $85-86 \%$, PPV of $35-36 \%$ and AUC of $0.69-0.74)$.

Results for the diagnostic performance of T2-weighted MR imaging, T2-weighted+DW MR imaging and ADC measurement for the prediction of axillary lymph node macrometastasis can be found in Table 3.3. ROC curves are presented in Figure 3.4. Examples of the imaging features of a benign and malignant lymph node is given in Figure 3.5 and 3.6.
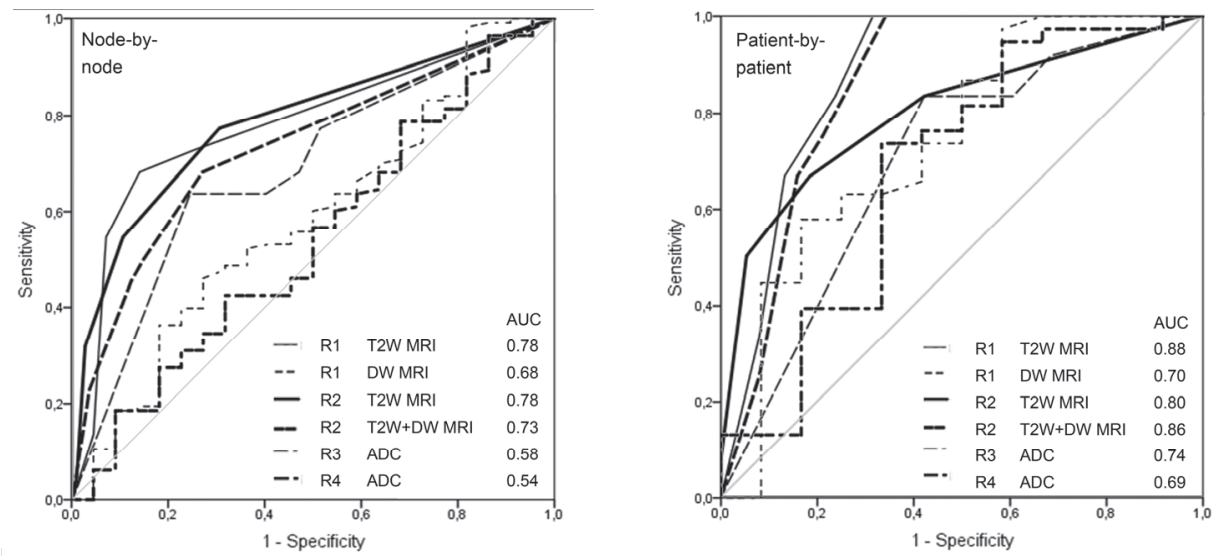

Figure 3.4 ROC curves for qualitative assessment T2-weighted (solid line), DW (dashed line) and T2-weighted+DW (dashed line) MR imaging for reader 1 (thin line) and reader 2 (bold line) and ROC curves for quantitative ADC measurement (dotted line) for reader 3 (thin dots) and 4 (bold dots). Reader 1 scored each lymph node again based on DW MR imaging, independently from the initial score based on T2-weighted MR imaging. Reader 2 only adjusted the initial score. 
Table 3.3 Diagnostic performance for prediction of axillary nodal status.

\begin{tabular}{|c|c|c|c|c|c|c|}
\hline & \multicolumn{2}{|c|}{ R1 } & \multicolumn{2}{|c|}{$\mathrm{R} 2$} & R3 & R4 \\
\hline & $\mathrm{T} 2 \mathrm{~W}$ & $\mathrm{DW}^{*}$ & $\mathrm{~T} 2 \mathrm{~W}$ & $T 2 W+D W^{*}$ & \multicolumn{2}{|c|}{ ADC } \\
\hline & \multicolumn{6}{|c|}{ Node-by-node (n=135) } \\
\hline Sensitivity & $55(12 / 22)$ & $64(14 / 22)$ & $32(7 / 22)$ & $45(10 / 22)$ & $41(9 / 22)$ & $41(9 / 22)$ \\
\hline $95 \% \mathrm{Cl}$ & $36-77$ & $45-82$ & $14-50$ & $27-68$ & 21-63 & 21-63 \\
\hline Specificity & $93(105 / 113)$ & $59(67 / 113)$ & $97(110 / 113)$ & $88(99 / 113)$ & $64(72 / 113)$ & $63(71 / 113)$ \\
\hline $95 \% \mathrm{Cl}$ & $88-97$ & $50-69$ & $94-100$ & 81-93 & $54-72$ & 53-72 \\
\hline PPV & $60(12 / 20)$ & $23(14 / 60)$ & $70(7 / 10)$ & $42(10 / 24)$ & $18(9 / 50)$ & $18(9 / 51)$ \\
\hline $95 \% \mathrm{Cl}$ & $42-79$ & $17-31$ & $42-100$ & 27-59 & $9-32$ & $9-31$ \\
\hline NPV & $91(105 / 115)$ & $89(67 / 75)$ & $88(110 / 125)$ & $89(99 / 111)$ & $85(72 / 85)$ & $85(71 / 84)$ \\
\hline $95 \% \mathrm{Cl}$ & 88-95 & $84-95$ & $85-91$ & $86-93$ & 75-91 & $75-91$ \\
\hline AUC & 0.78 & 0.68 & 0.78 & 0.73 & 0.58 & 0.54 \\
\hline $95 \% \mathrm{Cl}$ & $0.66-0.88$ & $0.57-0.79$ & $0.68-0.88$ & $0.62-0.84$ & $0.45-0.72$ & $0.40-0.67$ \\
\hline \multirow[t]{2}{*}{$P$ Value $^{+}$} & \multirow{2}{*}{\multicolumn{2}{|c|}{0.037}} & \multicolumn{2}{|c|}{0.368} & & \\
\hline & & & \multicolumn{2}{|c|}{ Patient-by-patient $(n=50)$} & & \\
\hline Sensitivity & $67(8 / 12)$ & $83(10 / 12)$ & $50(6 / 12)$ & $75(9 / 12)$ & $67(8 / 12)$ & $67(8 / 12)$ \\
\hline $95 \% \mathrm{Cl}$ & $35-89$ & $51-97$ & $22-78$ & $43-93$ & $35-88$ & $35-89$ \\
\hline Specificity & $87(33 / 38)$ & $50(19 / 38)$ & $95(36 / 38)$ & $84(32 / 38)$ & $63(24 / 38)$ & $61(23 / 38)$ \\
\hline $95 \% \mathrm{Cl}$ & 71-95 & $34-66$ & 81-99 & $68-93$ & 46-78 & 43-76 \\
\hline PPV & $62(8 / 13)$ & $34(10 / 29)$ & $75(6 / 8)$ & $60(9 / 15)$ & $36(8 / 22)$ & $35(8 / 23)$ \\
\hline $95 \% \mathrm{Cl}$ & $32-84$ & $19-54$ & $36-96$ & $33-83$ & $18-59$ & $17-57$ \\
\hline NPV & $89(33 / 37)$ & $90(19 / 21)$ & $86(36 / 42)$ & $91(32 / 35)$ & $86(24 / 28)$ & $85(23 / 27)$ \\
\hline $95 \% \mathrm{Cl}$ & $74-96$ & $68-98$ & 71-94 & 76-98 & $66-95$ & $65-95$ \\
\hline AUC & 0.88 & 0.70 & 0.80 & 0.86 & 0.74 & 0.69 \\
\hline $95 \% \mathrm{Cl}$ & $0.78-0.97$ & $0.56-0.84$ & $0.64-0.96$ & $0.76-0.96$ & $0.56-0.93$ & $0.49-0.89$ \\
\hline$P$ Value ${ }^{\ddagger}$ & \multicolumn{2}{|c|}{0.010} & \multicolumn{2}{|c|}{0.524} & & \\
\hline
\end{tabular}

$\mathrm{R} 1$, radiologist 1; $\mathrm{R} 2$, radiologist 2; $\mathrm{R} 3$, reader 3; $\mathrm{R} 4$, reader 4; $\mathrm{T} 2 \mathrm{~W}$, T2-weighted MR imaging; $\mathrm{DW}$, diffusionweighted MR imaging; ADC, apparent diffusion coefficient; $95 \% \mathrm{Cl}, 95 \%$ confidence interval; PPV, positive predictive value; NPV, negative predictive value; AUC, area under the curve;. number without parentheses indicates a percentage; numerator and denominator between parentheses indicate the absolute numbers for calculation of the parameter

* DW MR imaging was presented to the radiologists for visual assessment after T2-weighted MR imaging evaluation. R1 scored each lymph node again, independent from the initial score based on T2-weighted MR imaging. R2 used the additional information to adjust the initial score based on T2-weighted MR imaging. ${ }^{+} P$ Value for difference in AUC between T2-weighted and T2-weighted + DW MR imaging, based on bootstrap test $(n=1000)$. ${ }^{\ddagger} P$ Value for difference in AUC between T2-weighted and T2-weighted + DW MR imaging, based on DeLong's test.

\section{Discussion}

We found that T2-weighted axillary MR imaging shows good diagnostic performance (AUC 0.78 , based on node-by-node validation), with a NPV of $86-89 \%$ and a specificity of $87-95 \%$ on patient-level, for the prediction of axillary metastasis in patients with breast cancer. DW MR imaging, in addition to T2-weighted MR imaging, did not improve diagnostic performance (AUC 0.68-0.73). On patient level the NPV was comparable (90-91\%) and specificity inferior (50-84\%) to T2-weighted MR imaging only. In our study we performed dedicated axillary MR imaging, by using an imaging protocol 
with close contact between surface coil and the patient's axilla, specifically aimed at imaging of the axillary lymph nodes, instead of using a breast coil, and a T2-weighted sequence without fat suppression.
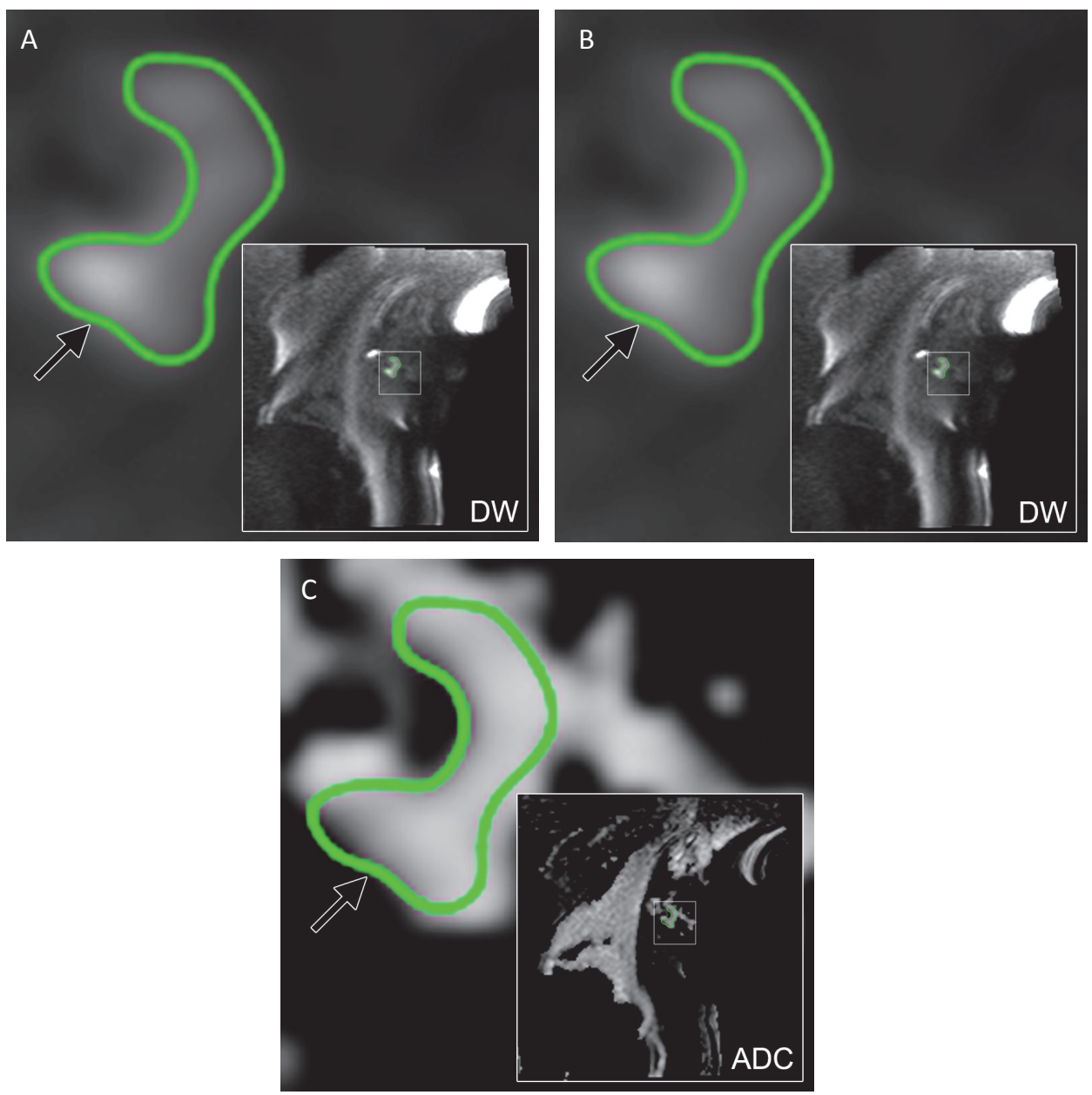

Figure 3.5 Example of a histopathologically proven benign lymph node (photographic enlargement) in left axilla, coronal plane (inset image). Woman aged 55 years with an invasive ductal carcinoma in the left breast, treated with lumpectomy and sentinel lymph node procedure (pT1NO). A: T2-weighted MR imaging with evident presence of the lymph node's fatty hilum. B: DW MR imaging (b value $800 \mathrm{sec} / \mathrm{mm}^{2}$ ) with the same lymph node depicted, with high diffusivity, reflected by relatively low signal intensity. C: Corresponding ADC map with high mean ADC value $\left(1.717 \times 10^{-3} \mathrm{~mm}^{2} / \mathrm{sec}\right)$, reflected by a high gray value.

The diagnostic performance of unenhanced axillary MR imaging was calculated on the basis of histopathologic findings of the surgically removed lymph nodes. Nonetheless, ITCs and micrometastasis were neglected and recorded as nonmalignant findings. This, 
given the fact that the clinical relevance of micrometastasis is questionable and the impact on adjuvant systemic therapy recommendations seems limited ${ }^{6,24-28}$. The calculation of the diagnostic performance based on patient-by-patient matching, in the case of SLNB, only included the radiological assessment of the lymph nodes which were (retrospectively) identified as the sentinel lymph nodes. This was done because the SLNB are shown to be false-negative in $8.61 \%$ (95\% Cl: 8.05-9.2\%) of the patients in the detection of additional metastasis in the axilla ${ }^{29}$.
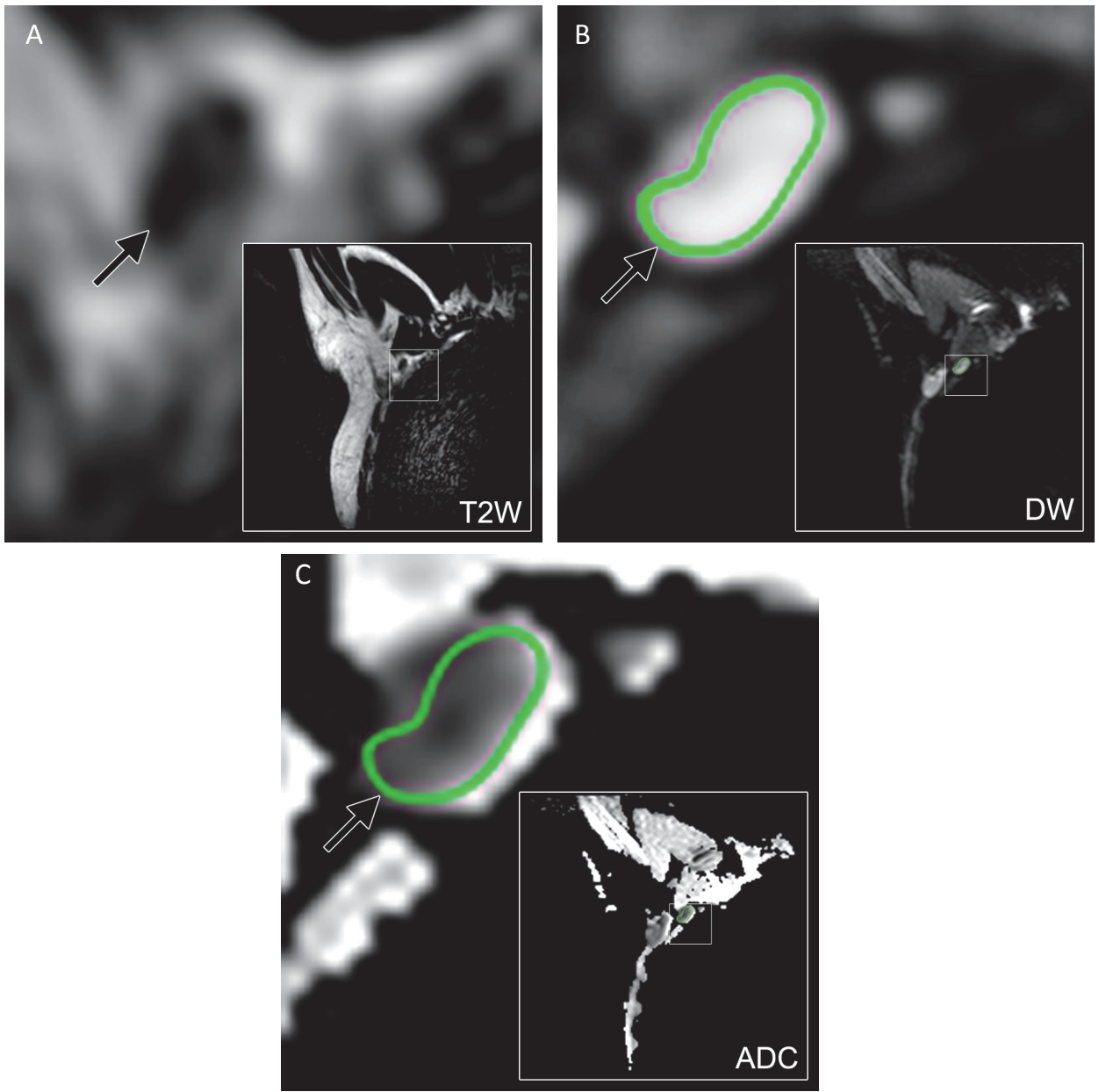

Figure 3.6 Example of a histopathologically proven malignant lymph node (photographic enlargement) in right axilla, coronal plane (inset image). Woman aged 64 years with an invasive ductal carcinoma in the right breast, treated with mastectomy and axillary lymph node dissection (pT2N1). A: T2-weighted MR imaging with absence of the lymph node's fatty hilum. B: DW MR imaging (b value $800 \mathrm{sec} / \mathrm{mm}^{2}$ ) with the same lymph node depicted, with low diffusivity, reflected by relatively high signal intensity. C: Corresponding ADC map with low mean ADC value $\left(0.624 \times 10^{-3} \mathrm{~mm}^{2} / \mathrm{sec}\right)$, reflected by a low gray value. 
The diagnostic performance in this study is comparable to previously published data of Scaranelo et al. ${ }^{13}$. In their study MR imaging showed a specificity of $82.4 \%$ with corresponding NPV of $91 \%$, while DW MR showed a specificity of $77.0 \%$ and NPV of $85 \%$. The study by Scaranelo had several limitations, as discussed by the authors. For example, axillary lymph nodes were assessed on standard breast MR imaging; it was assumed that the lymph node with the highest level of suspicion for cancer on the basis of the imaging criteria correlated with the overall surgical-pathologic diagnosis of the whole axilla; and the DW images had low resolving power, due to 1.5-T MR imaging and the $1000 \mathrm{sec} / \mathrm{mm}^{2} \mathrm{~b}$-value. Those limitations were limited in our study by using a dedicated axillary coil with 3.0-T MR imaging and performing node-by-node matching. Despite these improvements, the diagnostic performance of unenhanced MR imaging for nodal staging in breast cancer patients did not improve.

The addition of DW MR imaging to T2-weighted MR imaging did not result in an improved diagnostic performance, which is in line with previous studies on primary nodal staging in rectal cancer $^{30}$. Quantitative analysis of DW images by ADC measurement showed an AUC of 0.54-0.58. Therefore, visual analysis of DW images, in addition to T2-weighted images, can provide the radiologists of incorrect additional information on nodal status, leading to more incorrect nodal scoring compared to T2-weighted MR imaging alone. By adding DW MR imaging, more cases were scored as being positive for malignancy. We observed a higher increase in false-positive findings compared with the increase in true-positive findings and a decrease in true-negative findings, which resulted in reduced PPV and specificity, respectively. Only for R1 and not for R2, the difference in AUC between T2-weighted and T2-weighted+DW MR imaging was statistically significant, which can be explained by the difference in lymph nodes scoring strategy between the two readers and the higher diagnostic performance of T2-weighted MR imaging compared to ADC measurement.

Two different reading strategies were used by the two readers: one involved the use of the additional information from DW MR imaging to score each lymph node again, independent of the initial score based on T2-weighted MR images; and the other involved the use of DW MR imaging only for adjusting the initial T2-weighted MR imaging score. Since diagnostic performance of added DW MR imaging for both reading strategies was not sufficient for clinical implementation, we did not assess an analysis of intra- or inter-observer variability in this aspect of the analysis.

Possible explanations for insufficient diagnostic accuracy of DW MR imaging may include partial volume effects, since the lymph nodes are relatively small with diameters less than half a centimeter. Furthermore, the mean ADC might not reflect heterogeneity in diffusion within a single lymph node. Not only metastasis, but also reactive hyperplasia or fibrotic proliferation might affect diffusion. Finally, we included the $b$ value $0 \mathrm{sec} / \mathrm{mm}^{2}$ for ADC calculation, by using a mono-exponential fit, and therefore, perfusion contamination might have occurred. Since the upper b-value was $800 \mathrm{sec} / \mathrm{mm}^{2}$, noise contamination can be considered minimal. 
Since our study aim was to establish an alternative for the current invasive nodal staging procedure, the NPV should at least be noninferior to the NPV of SLNB, to safely omit SLNB if axillary MR images are scored as being negative for metastasis. A recent meta-analysis showed a false-negative rate of $8.61 \%$ (95\% CI: 8.05-9.2\%) of the SLNB ${ }^{29}$. Calculated NPV of the SLNB in a patient group with a prevalence of $24 \%$ of axillary metastasis, as in our study, equals $97.3 \%$. Compared to the NPV observed in our study (83-90\%), it can be concluded that both T2-weighted MR imaging and T2-weighted with added DW MR imaging cannot be substituted for SLNB for ruling out axillary metastasis and guide treatment decisions regarding axillary management.

Since axillary staging by ultrasound, followed by ultrasound-guided biopsy if indicated, shows high specificity (98.3\%; 95\% Cl 97.2-99.0\%) and high PPV $(97.1 \% ; 95 \% \mathrm{Cl}$ 95.2-98.3\%) $)^{31}$, unenhanced axillary MR imaging or T2-weighted with added DW MR imaging, showing PPVs up to 75\%, cannot replace ultrasound-guided biopsy staging technique for the detection of axillary metastasis.

SLNB is currently the most accurate method to rule out small micrometastatic nodal disease in breast cancer. We believe that these micrometastases remain beyond the morphological resolution of any clinically available MR technology. A lymph node specific contrast enhanced MR imaging could be of potential benefit, although further research is warranted. Gadofosveset-enhanced pelvic MR imaging for nodal staging in patients with rectal cancer showed high predictive values in two large patient cohorts with reported NPVs of $94-100 \%^{32,33}$. The report of a study in ten patient with breast cancer confirmed the high NPVs with gadofosveset MR contrast agent ${ }^{20}$.

In conclusion, unenhanced axillary MR imaging for nodal staging in patients with breast cancer showed a NPV of 86-91\%. Although this is considered good, it does not approach the NPV of SLNB and as a result it cannot be considered as a substitute for the current invasive staging procedure. Further research should focus on improving the diagnostic performance of axillary MR imaging for nodal staging in patients with breast cancer, for example by using lymph node specific contrast agents. 


\section{References}

1. Oliveira M, Cortes J, Bellet M, Balmana J, De Mattos-Arruda L, Gomez P, Munoz E, Ortega V, Perez J, Saura C, Vidal M, Rubio IT, Di Cosimo S. Management of the axilla in early breast cancer patients in the genomic era. Ann Oncol 2013;24:1163-70.

2. Pesce $\mathrm{C}$, Morrow M. The need for lymph node dissection in nonmetastatic breast cancer. Annu Rev Med 2013;64:119-29.

3. Galimberti V, Cole BF, Zurrida S, Viale G, Luini A, Veronesi P, Baratella P, Chifu C, Sargenti M, Intra M, Gentilini O, Mastropasqua MG, Mazzarol G, Massarut S, Garbay JR, Zgajnar J, Galatius H, Recalcati A, Littlejohn D, Bamert M, Colleoni M, Price KN, Regan MM, Goldhirsch A, Coates AS, Gelber RD, Veronesi $U$. Axillary dissection versus no axillary dissection in patients with sentinel-node micrometastases (IBCSG 23-01): a phase 3 randomised controlled trial. Lancet Oncol 2013;14:297-305.

4. Giuliano AE, Hunt KK, Ballman KV, Beitsch PD, Whitworth PW, Blumencranz PW, Leitch AM, Saha S, McCall LM, Morrow M. Axillary dissection vs no axillary dissection in women with invasive breast cancer and sentinel node metastasis: a randomized clinical trial. JAMA 2011;305:569-75.

5. Rutgers EJ, Donker M, Straver ME, Meijnen P, Van De Velde CJH, Mansel RE, Westenberg H, Orzalesi L, Bouma WH, van der Mijle H, Nieuwenhuijzen GAP, Veltkamp SC, Slaets L, Messina CGM, Duez NJ, Hurkmans C, Bogaerts J, van Tienhoven G. Radiotherapy or surgery of the axilla after a positive sentinel node in breast cancer patients: Final analysis of the EORTC AMAROS trial (10981/22023). J Clin Oncol 2013. 31(suppl; abstr LBA1001).

6. van Roozendaal LM, Schipper RJ, Van de Vijver KK, Haekens CM, Lobbes MB, Tjan-Heijnen VC, de Boer $M$, Smidt ML. The impact of the pathological lymph node status on adjuvant systemic treatment recommendations in clinically node negative breast cancer patients. Breast Cancer Res Treat 2014;143:469-76.

7. Schijven MP, Vingerhoets AJ, Rutten HJ, Nieuwenhuijzen GA, Roumen RM, van Bussel ME, Voogd AC. Comparison of morbidity between axillary lymph node dissection and sentinel node biopsy. Eur J Surg Oncol 2003;29:341-50.

8. DiSipio T, Rye S, Newman B, Hayes S. Incidence of unilateral arm lymphoedema after breast cancer: a systematic review and meta-analysis. Lancet Oncol 2013;14:500-15.

9. Mortellaro VE, Marshall J, Singer L, Hochwald SN, Chang M, Copeland EM, Grobmyer SR. Magnetic resonance imaging for axillary staging in patients with breast cancer. J Magn Reson Imaging 2009; 30:309-12.

10. Kvistad KA, Rydland J, Smethurst HB, Lundgren S, Fjøsne HE, Haraldseth O. Axillary lymph node metastases in breast cancer: preoperative detection with dynamic contrast-enhanced MRI. Eur Radiol 2000;10:1464-71.

11. Cooper KL, Harnan S, Meng Y, Ward SE, Fitzgerald P, Papaioannou D, Wyld L, Ingram C, Wilkinson ID, Lorenz E. Positron emission tomography (PET) for assessment of axillary lymph node status in early breast cancer: A systematic review and meta-analysis. Eur J Surg Oncol 2011;37:187-98.

12. Harnan SE, Cooper KL, Meng Y, Ward SE, Fitzgerald P, Papaioannou D, Ingram C, Lorenz E, Wilkinson ID, Wyld L. Magnetic resonance for assessment of axillary lymph node status in early breast cancer: a systematic review and meta-analysis. Eur J Surg Oncol 2011;37:928-36.

13. Scaranelo AM, Eiada R, Jacks LM, Kulkarni SR, Crystal P. Accuracy of unenhanced MR imaging in the detection of axillary lymph node metastasis: study of reproducibility and reliability. Radiology 2012; 262:425-34.

14. He N, Xie C, Wei W, Pan C, Wang W, Lv N, Wu P. A new, preoperative, MRI-based scoring system for diagnosing malignant axillary lymph nodes in women evaluated for breast cancer. Eur J Radiol 2012;81:2602-12.

15. Fornasa F, Nesoti MV, Bovo C, Bonavina MG. Diffusion-weighted magnetic resonance imaging in the characterization of axillary lymph nodes in patients with breast cancer. J Magn Reson Imaging 2012; 36:858-64.

16. Luo N, Su D, Jin G, Liu L, Zhu X, Xie D, Liu Y. Apparent diffusion coefficient ratio between axillary lymph node with primary tumor to detect nodal metastasis in breast cancer patients. J Magn Reson Imaging 2013;38:824-8. 
17. Kamitani T, Hatakenaka M, Yabuuchi H, Matsuo Y, Fujita N, Jinnouchi M, Nagao M, Shirahane K, Tokunaga $\mathrm{E}$, Honda $\mathrm{H}$. Detection of axillary node metastasis using diffusion-weighted $\mathrm{MRI}$ in breast cancer. Clin Imaging 2013;37:56-61.

18. Baltzer PA, Dietzel M, Burmeister HP, Zoubi R, Gajda M, Camara O, Kaiser WA. Application of MR mammography beyond local staging: is there a potential to accurately assess axillary lymph nodes? evaluation of an extended protocol in an initial prospective study. AJR Am J Roentgenol 2011;196: W641-7.

19. Luciani A, Dao TH, Lapeyre M, Schwarzinger M, Debaecque C, Lantieri L, Revelon G, Bouanane M, Kobeiter H, Rahmouni A. Simultaneous bilateral breast and high-resolution axillary MRI of patients with breast cancer: preliminary results. AJR Am J Roentgenol 2004;182:1059-67.

20. Schipper RJ, Smidt ML, van Roozendaal LM, Castro CJ, de Vries B, Heuts EM, Keymeulen KB, Wildberger JE, Lobbes MB, Beets-Tan RG. Noninvasive nodal staging in patients with breast cancer using gadofosveset-enhanced magnetic resonance imaging: a feasibility study. Invest Radiol 2013;48: 134-9.

21. Lester SC, Bose S, Chen YY, Connolly JL, de Baca ME, Fitzgibbons PL, Hayes DF, Kleer C, O'Malley FP, Page DL, Smith BL, Tan LK, Weaver DL, Winer E; Members of the Cancer Committee, College of American Pathologists. Protocol for the examination of specimens from patients with invasive carcinoma of the breast. Arch Pathol Lab Med 2009;133:1515-38.

22. Efron B. Bootstrap Methods: Another Look at the Jackknife. Ann Statist 1979;7:1-26.

23. DeLong ER, DeLong DM, Clarke-Pearson DL. Comparing the areas under two or more correlated receiver operating characteristic curves: a nonparametric approach. Biometrics 1988;44: 837-45.

24. Giuliano AE, Hawes D, Ballman KV, Whitworth PW, Blumencranz PW, Reintgen DS, Morrow M, Leitch AM, Hunt KK, McCall LM, Abati A, Cote R. Association of occult metastases in sentinel lymph nodes and bone marrow with survival among women with early-stage invasive breast cancer. JAMA 2011;306: 385-93.

25. Gobardhan PD, Elias SG, Madsen EV, van Wely B, van den Wildenberg F, Theunissen EB, Ernst MF, Kokke MC, van der Pol C, Borel Rinkes IH, Wijsman JH, Bongers V, van Gorp J, van Dalen T. Prognostic value of lymph node micrometastases in breast cancer: a multicenter cohort study. Ann Surg Oncol 2011;18:1657-64.

26. Maaskant-Braat AJ, van de Poll-Franse LV, Voogd AC, Coebergh JW, Roumen RM, Nolthenius-Puylaert MC, Nieuwenhuijzen GA. Sentinel node micrometastases in breast cancer do not affect prognosis: a population-based study. Breast Cancer Res Treat 2011;127:195-203.

27. Weaver DL, Ashikaga T, Krag DN, Skelly JM, Anderson SJ, Harlow SP, Julian TB, Mamounas EP, Wolmark N. Effect of occult metastases on survival in node-negative breast cancer. N Engl J Med 2011;364: 412-21.

28. de Boer M, van Deurzen CH, van Dijck JA, Borm GF, van Diest PJ, Adang EM, Nortier JW, Rutgers EJ, Seynaeve C, Menke-Pluymers MB, Bult P, Tjan-Heijnen VC. Micrometastases or isolated tumor cells and the outcome of breast cancer. N Engl J Med 2009;361:653-63.

29. Pesek S, Ashikaga T, Krag LE, Krag D. The false-negative rate of sentinel node biopsy in patients with breast cancer: a meta-analysis. World J Surg 2012;36:2239-51.

30. Heijnen LA, Lambregts DM, Mondal D, Martens MH, Riedl RG, Beets GL, Beets-Tan RG. Diffusionweighted MR imaging in primary rectal cancer staging demonstrates but does not characterise lymph nodes. Eur Radiol 2013;23:3354-60.

31. Houssami N, Ciatto S, Turner RM, Cody HS 3rd, Macaskill P. Preoperative ultrasound-guided needle biopsy of axillary nodes in invasive breast cancer: meta-analysis of its accuracy and utility in staging the axilla. Ann Surg 2011;254:243-51.

32. Lambregts DM, Beets GL, Maas M, Kessels AG, Bakers FC, Cappendijk VC, Engelen SM, Lahaye MJ, de Bruïne AP, Lammering G, Leiner T, Verwoerd JL, Wildberger JE, Beets-Tan RG. Accuracy of gadofosveset-enhanced MRI for nodal staging and restaging in rectal cancer. Ann Surg 2011;253: 53945.

33. Heijnen LA, Lambregts DM, Martens MH, Maas M, Bakers FC, Cappendijk VC, Oliveira P, Lammering G, Riedl RG, Beets GL, Beets-Tan RG. Performance of gadofosveset-enhanced MRI for staging rectal cancer nodes: can the initial promising results be reproduced? Eur Radiol 2014;24:371-9. 


\section{Chapter 4}

Noninvasive nodal staging in breast cancer patients using gadofosveset-enhanced magnetic resonance imaging: a feasibility study

RJ. Schipper

M.L. Smidt

L.M. van Roozendaal

C.J. Castro

B. de Vries

E.M. Heuts

K.B.M.I. Keymeulen

J.E. Wildberger

M.B.I. Lobbes

R.G.H. Beets-Tans

Invest Radiol 2013;48:134-9 


\section{Abstract}

\section{Objectives}

The objectives of this study was to evaluate whether the axillary lymph nodes show enhancement on magnetic resonance imaging (MRI) after gadofosveset administration, to assess the time to peak enhancement, and to determine the diagnostic performance of gadofosvesetenhanced MRI for axillary nodal staging.

\section{Material and methods}

Ten women whose conditions had been diagnosed with invasive breast cancer $(>2 \mathrm{~cm})$ underwent both nonenhanced and gadofosveset-enhanced 3-dimentional T1-weighted axillary MRI. Signal intensity of the axillary lymph nodes and different adjacent tissues was measured and relative signal intensity ( $\mathrm{rSI}$ ) was calculated. A Wilcoxon signed rank test was used to compare results of $\mathrm{rSI}$ between different time intervals. A radiologist evaluated all lymph nodes with regard to size, morphologic features and gadofosveset uptake. All MRI depicted lymph nodes were matched with lymph nodes that were removed during surgery. Nodal status was investigated by a pathologist. Sensitivity, specificity, positive predictive value (PPV), and negative predictive value (NPV) of gadofosveset-enhanced MRI for axillary lymph node staging were calculated.

\section{Results}

After contrast administration, a significant signal increase was observed in the lymph nodes $(p<0.05)$. When compared with muscle or fat, $\mathrm{rSI}$ of the lymph nodes demonstrated a significant postcontrast peak enhancement between 11 minutes and 30 seconds and 20 minutes and 50 seconds $(P<0.05)$. A total of 152 lymph nodes were harvested during sentinel lymph node biopsy or axillary lymph node dissection, of which 116 were matched with the lymph nodes that were depicted on MRI. Histopathological examination resulted in 21 macro-metastases and 8 micro-metastases. Using contrast-enhanced MRI, 20 lymph nodes were rated as true positive, 83 as true-negative, 4 as false positive, and 9 as false negative. This resulted in an overall nodeby-node sensitivity, specificity, PPV and NPV of $69 \%, 95 \%, 83 \%$ and $90 \%$, respectively. If micrometastases were excluded from analysis, MRI showed a sensitivity of $86 \%$ and a specificity of $94 \%$. Calculated PPV and NPV were $75 \%$ and $97 \%$, respectively.

\section{Conclusion}

The axillary lymph nodes show enhancement on MRI after gadofosveset administration, with peak enhancement between 11 minutes and 30 seconds and 20 minutes and 50 seconds. Diagnostic performance of gadofosveset-enhanced axillary lymph node imaging in patients with breast cancer is promising, but further studies need to confirm these results. 


\section{Background}

With an estimated number of 1,383,500 patients whose conditions have been newly diagnosed worldwide, breast cancer is the number 1 cancer in women ${ }^{1}$. The basic treatment of stages T1 to T2 breast cancer with a clinically negative axillary lymph node status consists of tumor excision and nodal staging with sentinel lymph node biopsy (SLNB), followed by radiotherapy and systemic therapy, if indicated. The treatment of the axilla is performed to achieve staging, local control, and possible survival benefit. Current nodal workup in patients with breast cancer consists of axillary ultrasound (US) (with or without tissue sampling) and, in case of no metastasis, followed by SLNB. In case of nodal metastasis, a complementary axillary lymph node dissection (cALND) is performed. Complementary axillary lymph node dissection is associated with significant morbidity; the most reported are arm and shoulder pain, lymphedema, nerve injury and shoulder stiffness. Similar, clinically relevant complications are also reported after SLNB, although less frequently ${ }^{2,3}$. In conclusion, the current workup is extensive and not without any harm.

Until recently, the consensus consisted about the need to detect and treat every single axillary metastasis. The benefit of this approach was already questioned by Fisher et al, who compared omission versus performance of axillary lymph node dissection (ALND) in patients with breast cancer ${ }^{4}$. In this study (National Surgical Adjuvant Breast and Bowel project-4), ALND did not result in a survival benefit, not even after a follow up of 25 years. In addition, another randomized clinical trial (American College of Surgeons Oncology Group-Z0011) compared cALND to a "wait-and-see" policy in women with T1 to T2 breast cancer and sentinel node metastases who were treated by breast conserving therapy. At a median follow-up of 6.3 years, 5 -year survival was $91.8 \%$ with CALND and $92.5 \%$ with SNLB alone. It was concluded that omission of the CALND in this patient population did not result in inferior survival results ${ }^{5}$.

These insights create opportunities for research on axillary lymph node staging in patients with breast cancer through noninvasive (contrast-enhanced) magnetic resonance imaging (MRI).

A meta-analysis concerning noninvasive nodal staging in breast cancer concluded that ultrasmall superparamagnetic iron oxide(USPIO)-enhanced MRI provided the highest sensitivity and specificity for axillary lymph node metastases ${ }^{6}$. However, USPIO is not approved by the Food and Drug Administration. Recent publication on contrastenhanced MRI for lymph node staging in patients with rectal cancer using gadofosveset as contrast agent showed promising results ${ }^{7,8}$. Gadofosveset-enhanced MRI showed a high negative predictive value (NPV) for nodal metastases detection similar to that of USPIO-enhanced $\mathrm{MRI}^{8}$. It was hypothesized that gadofosveset-enhanced MRI of the 
axilla might provide useful information about nodal status in patients with breast cancer.

The primary aim of this study were to evaluate whether the axillary lymph nodes in patients with breast cancer showed enhancement on MRI after gadofosveset administration and to assess the optimal post-contrast imaging period (i.e. peak enhancement). The second aim was to determine the diagnostic performance of gadofosveset-enhanced MRI for axillary staging in a restricted patient group.

\section{Materials and methods}

\section{Setting and patients}

This prospective single-center study was approved by the local ethical committee and all patients gave written informed consent (Medical Ethical Committee decision number 11-2-016). Ten patients with histopathological confirmed invasive breast cancer who were about to undergo nodal staging were included. The inclusion criteria were as follows: patients with clinically palpable lymph nodes or with nodes suspicious for metastasis with axillary US. The exclusion criteria were as follows: history of neoadjuvant chemotherapy for breast cancer or radiotherapy of the axilla or surrounding areas of the axilla, prior axillary surgery, and contra-indications for either MRI or the gadofosveset contrast agent.

\section{Patients routing}

In our hospital, patients with breast cancer undergo breast MRI with a gadoliniumbased contrast agent for pre-operative local staging. Axillary gadofosveset-enhanced MRI was performed at least 72 hours later. Ultrasound-guided core biopsy of the lymph node using a 16-gauge needle was performed immediately after axillary MRI. Patient routing is summarized in Figure 4.1.

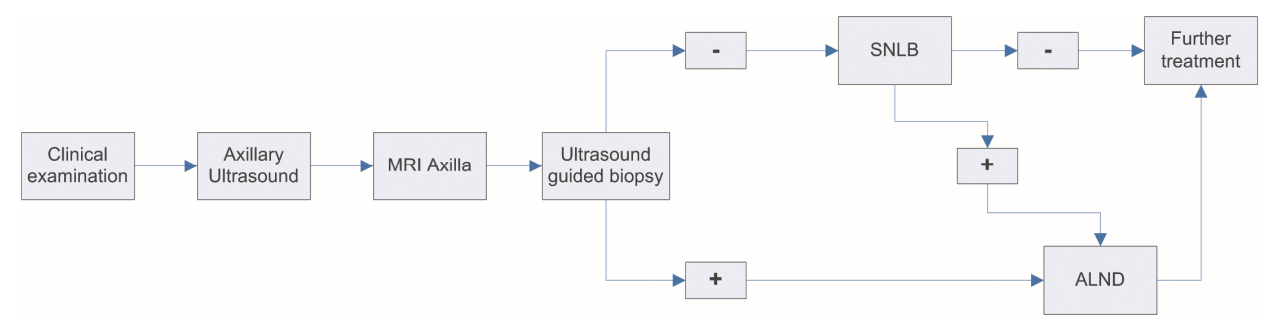

Figure 4.1 Patient routing. The ultrasound guided axillary lymph node biopsy was performed after the axillary MRI. 


\section{Imaging}

Axillary imaging was performed on a 3-T MRI scanner (Achieva 3.0 TX, Philips Healthcare, Best, The Netherlands) using a 32-channel cardiac sensitivity encoding coil with the patient in a supine position and with the arm elevated. Our sequence protocol consisted of the following parameters: 3-dimensional T1-weighted gradient-echo sequence (repetition time/echo time 6.3/4.6 milliseconds; flip angle, 15 degrees; number of signal averages 2; slice thickness, $1.25 \mathrm{~mm}$; field-of-view, 220x220; matrix size, $176 \times 176$, resulting in an in-plane resolution of $1.25 \times 1.25 \mathrm{~mm}^{2}$; and temporal resolution, 2 minutes and 50 seconds). The protocol contained a nonenhanced 3-dimensional T1 weighted sequence, which was repeated 8 times after contrast administration.

\section{Contrast agent}

Gadofosveset is an intravascular blood-pool magnetic resonance (MR) contrast agent that binds to albumin, which was originally designed for vascular MR imaging. Because lymph fluid is rich in albumin, it proved to be useful for lymph node visualization ${ }^{9}$. Gadofosveset (Ablavar ${ }^{\odot}$, Lantheus Medical Imaging, North Billerica, United States of America) was administered via automatic bolus injection in the antecubital vein through an intravenous line. The dose was $0.12 \mathrm{ml} / \mathrm{kg}$ of body weight, which was injected at $2.0 \mathrm{ml} / \mathrm{s}$, followed by a $25 \mathrm{ml}$ saline flush.

\section{Image evaluation}

Signal intensity (SI) was measured on different locations (lymph node $\left(\mathrm{SI}_{\mid \mathrm{n}}\right)$, latissimus dorsi muscle $\left(\mathrm{SI}_{\text {muscle }}\right)$, axillary fat $\left(\mathrm{SI}_{\mathrm{fat}}\right)$ and axillary artery $\left.\left(\mathrm{SI}_{\text {artery }}\right)\right)$ on all 9 time points. Signal intensity was used to determine the optimal waiting time between contrast administration and imaging. Signal intensity ratios were calculated using the following the ratios: $\mathrm{SI}_{\mid n} / \mathrm{SI}_{\text {muscle, }} \mathrm{SI}_{I_{n}} / \mathrm{SI}_{\text {fat }}$ and $S I_{\mid n} / S I_{\text {artery }}$. The highest ratio between the $\mathrm{SI}_{\mid \mathrm{n}}$ and $\mathrm{SI}_{\text {muscle }}$ and/or $\mathrm{SI}_{\text {fat }}$ was defined as peak enhancement.

After the determination of the optimal interval, this time point was used for further evaluation of diagnostic performance of this approach. All images, both nonenhanced and enhanced, were evaluated by a radiologist, who was blinded for all clinical data and histological results. This radiologist has 6 years experience in reading gadofosvesetenhanced MRI in patients with rectal cancer for lymph node staging. First all observed lymph nodes on MR were drawn on an anatomical map consisting of 3 schematic coronal slices of the axilla. Thereafter, lymph nodes were classified using a confidence level score ( 0 =certainly not malignant, 1 =probably not malignant, $2=$ =possibly malignant, $3=$ probably malignant, or $4=$ certainly malignant). The score was based on the criteria as described by Lambregts et al. ${ }^{10}$; a node was considered as benign when the nodes show significant contrast hyperintensity after intravenous injection of gadofosveset contrast. The best criteria to detect malignant nodes are the absence of contrast hyperintensity 
and the absence of an intact nodal border on gadofosveset-enhanced MRI. A malignant lymph node on gadofosveset-enhanced MRI is illustrated in Figure 4.2.
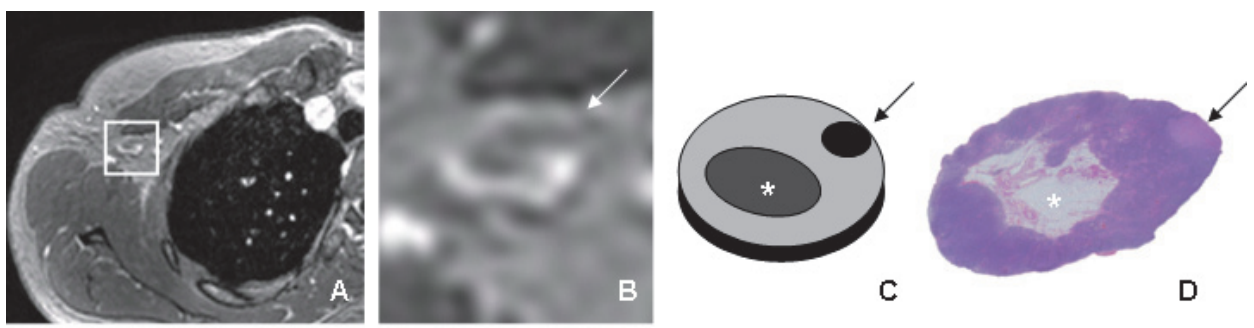

Figure 4.2 Malignant lymph node on gadofosveset-enhanced MRI. A: An enlarged lymph node with a bright adjacent blood vessel (open rectangle). B: A close view on the lymph node in the open rectangle. C: Schematic view of the lymph node, the lymph node hilus $\left(^{*}\right)$ showed less enhancement (benign), however in the cortex a clear interruption (arrow) in the enhancement of the cortex is visible. D: Histopathological confirmation of a malignant lymph node (arrow).

\section{Surgery}

The sentinel lymph node (SLN) was identified by using technetium-99m nanocolloid as a radioactive tracer before surgery and 1 to $2 \mathrm{ml}$ of blue dye (Bleu Patente V; Guerbet, Aulnay-sous-Bois, France) for lymphatic mapping. During surgery, the SLN was harvested guided by the triple technique consisting of preoperative lymphoscintigraphy, blue lymphatic vessels, and detection of radioactivity by using gamma probe.

During the levels 1 and 2 ALND, the following anatomical landmarks were marked: first the caudal-medial edge of specimen, followed by the caudal-lateral and the craniallateral edges. In addition a marker was placed at the branching of the lateral thoracic vein from the axillary vein. Finally, the medial apex of axilla was marked.

\section{Histopathological evaluation}

Histological evaluation of harvested lymph nodes took place according to the following protocols. 1 protocol for the nodes removed during the SLNB procedure and 1 protocol for the nodes harvested from an ALND.

The SLNs were sliced with a maximum slice thickness of $3 \mathrm{~mm}$ and embedded in paraffin. Each part was step sectioned at 500-micrometer intervals at 3 levels and stained with haematoxylin and eosin (H\&E). Cytokeratin immunohistochemical staining (IHC), using a mouse anti-human MNF116 antibody (Dako), was performed if indicated.

After the surgery, ALND specimens were received immediately from the operating room. Before formalin fixation, the specimen was fixed in a box representing the normal anatomical situation in the patient (see Picture 4.1). After 24 hours of fixation 
all palpable lymph nodes were embedded in paraffin. Lymph nodes bigger than $5 \mathrm{~mm}$ were sliced with a maximum slice thickness of $3 \mathrm{~mm}$, and stained with $\mathrm{H} \& \mathrm{E}$, and IHC (if indicated). After microscopic examination, each node was recorded as benign, or isolated tumor cell $(I T C)(\mathrm{pNO}(\mathrm{i}+))(\leq 0.2 \mathrm{~mm})$, or micro metastasis $(\mathrm{pN} 1 \mathrm{mi})(0.2 \leq 2.0 \mathrm{~mm})$ or macro metastasis ( $\mathrm{pN} 1)(>2.0 \mathrm{~mm})$.

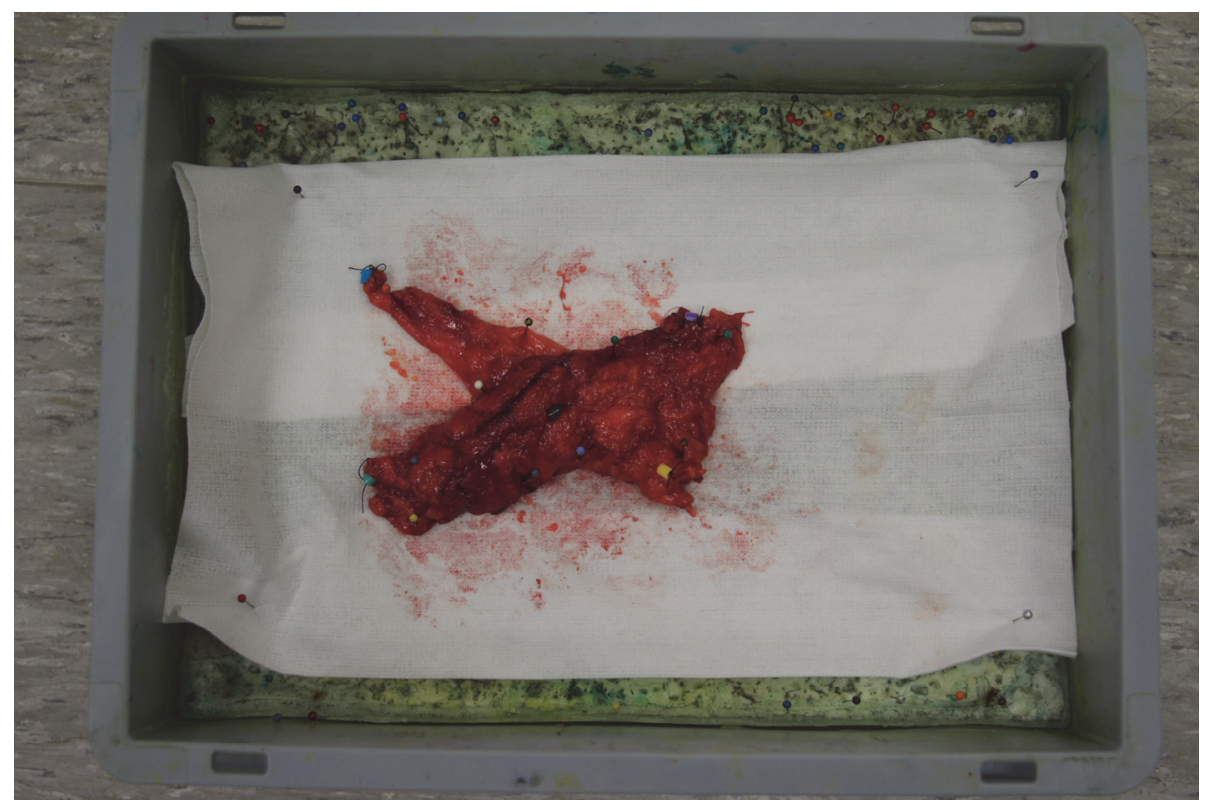

Picture 4.1 A left side axillary lymph nodes dissection specimen was placed into a box before fixation with formalin. The specimen was fixed in the box to the anatomical situation during axillary MRI.

\section{Node-by-node evaluation}

According to current guidelines only patients whose conditions have been diagnosed as node positive after axillary US with biopsy or fine needle aspiration biopsy (FNAB) were treated with ALND. All other patients underwent an SLNB procedure. To achieve a reliable node-by-node match in patients undergoing a SLNB, a single photon emission computed tomography-X-ray computed tomography (SPECT-CT) scan was used for matching of the SLNs removed during surgery and imaged by MRI. To achieve a reliable match between the ALND specimen and MRI, the anatomical map was used. Every node harvested by the pathologist during sampling was drawn on a separate anatomical map. Subsequently, the anatomical map based on MRI and the anatomical map based on the ALND specimen were compared and node-by-node matching was performed by the radiologist and pathologist side by side. 


\section{Statistical analyses}

Statistical analyses were performed using Statistical Package for the Social Sciences (version 18.0; SPSS Chicago, Illinois). The Wilcoxon signed rank test was used to compare the results of the SI-ratio's calculations before and after gadofosveset administration. Sensitivities, specificities, PPVs, and NPVs with $95 \%$ confidence intervals for axillary lymph node staging were calculated on a node-by-node base. In addition, the sensitivity and specificity on a patient-to-patient base for nodal staging was calculated. $\mathrm{P}<0.05$ was considered statistically significant.

\section{Results}

\section{Patients}

Ten patients (all women) were included in this study, with a median age of 48 years (range 31-81 years). All conditions of the patients were diagnosed as unilateral invasive breast cancer. In five patients, axillary metastasis was detected after US guided biopsy and ALND was performed instantly. The remaining patients underwent SLNB, revealing another two patients with axillary metastasis. In these patients, a cALND was performed. Invasive ductal carcinoma was the most common type of breast cancer $(n=8)$. One patient had invasive lobular carcinoma, whereas the remaining patient had invasive micro papillary carcinoma. Further patient and tumor characteristics are summarized in Table 4.1. Median time between axillary MRI and surgery was 20 days (range 6-29). During gadofosveset administration no adverse reactions were observed in all ten patients.

\section{Peak enhancement}

After contrast administration, a significant signal increase was observed in the axillary lymph nodes. In Table 4.2, SI ratios at different time points are presented. Using the Wilcoxon signed rank test, a significant peak enhancement within the nodes was observed at time interval $t=5, t=6$ and $t=7$ (i.e. 11 minutes and 30 seconds and 20minutes and 50seconds after gadofosveset administration). 
Table 4.1 Patient and tumor characteristics.

\begin{tabular}{|c|c|c|}
\hline Characteristics & & Numbers \\
\hline Age (mean, range) & & 50 years $(31-81)$ \\
\hline \multirow[t]{3}{*}{ Type cancer } & Invasive ductal carcinoma & 8 \\
\hline & Invasive lobular carcinoma & 1 \\
\hline & Invasive micro papillary carcinoma & 1 \\
\hline Size (mean, range) & & $3.5 \mathrm{~cm}(1.2-9.0)$ \\
\hline \multirow[t]{3}{*}{ Grade $^{1}$} & 1 & 0 \\
\hline & 2 & 4 \\
\hline & 3 & 6 \\
\hline \multirow[t]{2}{*}{ Multifocal } & Yes & 1 \\
\hline & No & 9 \\
\hline ER/PR + Her2/neu - (n) & & 6 \\
\hline Triple negative $(n)$ & & 2 \\
\hline$E R / P R+$ Her2/neu + (n) & & 2 \\
\hline
\end{tabular}

ER: estrogen receptor; PR: progesterone receptor. Her2-/neu: human epidermal growth factor receptor. ${ }^{1}$ Tumor grade following Bloom and Richardson.

Table 4.2 Lymph nodes enhancement.

\begin{tabular}{|c|c|c|c|c|c|c|}
\hline & ${ }_{r} S I_{\ln / \text { muscle }}$ & P-value & ${ }_{r} S I_{l n} / S I_{\text {fat }}$ & P-value & ${ }_{r} \mathrm{SI}_{\ln } / \mathrm{SI}_{\text {art. }}$ & P-value \\
\hline $\mathrm{T}=0$ & $1.4 \pm 0.5$ & & $0.8 \pm 0.2$ & & $1.4 \pm 0.3$ & \\
\hline \multicolumn{7}{|c|}{ Gadofosveset administration } \\
\hline T1 & $1.6 \pm 0.5$ & ns & $1.1 \pm 0.2$ & $\leq 0.01$ & $0.5 \pm 0.1$ & $\leq 0.01$ \\
\hline $\mathrm{T} 2$ & $1.6 \pm 0.5$ & 0.03 & $1.1 \pm 0.3$ & $\leq 0.01$ & $0.5 \pm 0.1$ & $\leq 0.01$ \\
\hline T3 & $1.6 \pm 0.4$ & ns & $1.1 \pm 0.1$ & $\leq 0.01$ & $0.5 \pm 0.1$ & $\leq 0.01$ \\
\hline T4 & $1.55 \pm 0.4$ & ns & $1.1 \pm 0.2$ & $\leq 0.01$ & $0.6 \pm 0.1$ & $\leq 0.01$ \\
\hline T5 & $1.51 \pm 0.4$ & 0.04 & $1.1 \pm 0.2$ & $\leq 0.01$ & $0.5 \pm 0.1$ & $\leq 0.01$ \\
\hline T6 & $1.63 \pm 0.6$ & 0.02 & $1.1 \pm 0.2$ & $\leq 0.01$ & $0.6 \pm 0.1$ & $\leq 0.01$ \\
\hline $\mathrm{T7}$ & $1.62 \pm 0.6$ & 0.02 & $1.1 \pm 0.2$ & $\leq 0.01$ & $0.6 \pm 0.1$ & $\leq 0.01$ \\
\hline T8 & $1.52 \pm 0.5$ & ns & $1.1 \pm 0.2$ & $\leq 0.01$ & $0.6 \pm 0.1$ & $\leq 0.01$ \\
\hline
\end{tabular}

Data are means \pm standard deviations. P-values were calculated for before and after gadofosveset enhancement. SI: signal intensity, In: lymph node, art.: artery.

\section{Lymph nodes}

During 5 SLNB procedures a total of 7 SLNs and 4 non-SLNs were removed. In 2 patients a macro metastasis was detected and CALND was performed. Histopathological evaluation of 7 (c)ALND specimens revealed a total of 141 lymph nodes (mean 20, range $9-28)$.

In total, 152 lymph nodes were harvested at the histologic examination of the specimens, of which 116 were matched with lymph nodes that were depicted on MRI, and a node-by-node analysis was performed. Consequently, 36 lymph nodes were not depicted on MRI or could not be matched. In patients treated with SLNB only, MRI depicted 22 additional lymph nodes, in which pathological matching was impossible. All these 22 nodes were considered as negative on MRI in the patient-to-patient analysis. The histopathological examination of the matched lymph nodes resulted in 21 macro 
metastases (mean size $8.4 \mathrm{~mm}$, range 2.2-22.0 mm) and 8 micro metastases (mean size $0.8 \mathrm{~mm}$, range $0.4-2.0 \mathrm{~mm}$ ). There were no nodes with ITC metastases.

On the basis of the results acquired by contrast-enhanced MRI, 20 lymph nodes were rated as true positive, 83 as true negative, 4 as false positive, and 9 as false negative. This resulted in an overall node-to-node sensitivity, specificity, PPV and NPV of $69 \%$ (95\% Cl 49-84), 95\% (95\% Cl 88-99), 83\% (95\% Cl 62-95) and 90\% (95\% Cl 81-95), respectively. If micro metastases are excluded, $18 \mathrm{lymph}$ nodes were rated as true positive, 89 as true negative, 6 as false positive, and 3 as false negative, resulting in a sensitivity of $86 \%(95 \% \mathrm{Cl} 63-96)$ and a specificity of $94 \%$ (95\% Cl 86-97). Calculated PPV and NPV was 75\% (95\% Cl 53-89) and $97 \%$ (95\% Cl 90-99).

After translating the node-by-node results to a more clinical relevant patient-by-patient comparison, contrast enhanced MRI resulted in seven true positive patients, two false positive and one true negative. This resulted in a sensitivity and specificity of $100 \%$ and $33 \%$ on a per patient basis, respectively.

Furthermore, we compared the diagnostic performance of axillary US alone, axillary US with tissue sampling and gadofosveset enhanced axillary MRI in predicting the pathological nodal status following the TNM classification. The axillary US predicted the correct pathological nodal status in three patients, overstaged it in four and understaged it in three patients. When using axillary US with tissue sampling (in all ten patients core biopsy was performed), pathological nodal status was predicted correct in five patients, one patient was overstaged and four understaged. Finally, gadofosveset enhanced axillary MRI predicted the correct pathological nodal status in eight patients and overstaged it in the remaining two patients.

\section{Discussion}

This study assessed the feasibility of noninvasive axillary staging in patients with breast cancer using gadofosveset-enhanced axillary MRI. It demonstrated that intravenous administration of gadofosveset showed enhancement of axillary lymph nodes with a peak enhancement between 11 minutes and 30 seconds and 20 minutes and 50 seconds. With the use of gadofosveset-enhanced MRI, a sensitivity of $86 \%(95 \% \mathrm{Cl}$ $63-96)$ and a specificity of $94 \%(95 \% \mathrm{Cl} 86-97)$ for axillary lymph node staging were achieved.

Nodal staging in patients with breast cancer has evolved from standard ALND to physical examination and US of the axilla and if negative, SLNB. About 30 to $40 \%$ of patients whose conditions are newly diagnosed as breast cancer have nodal metastases $^{11}$. The accuracy of physical examination of the axilla for pre-operative staging is low, with a sensitivity of 25 to $32.3 \%$ for detecting axillary metastases ${ }^{12-14}$. If, on axillary US, a node is suspicious for malignancy (often based on cortical thickening, effacement or replacement of the fatty hilum and size), a FNAB or core biopsy is 
performed. The sensitivity of axillary US combined with FNAB or core biopsy varies from 30.6 to $63.2 \%$, with a specificity of $100 \%{ }^{15}$. If a biopsy contains metastasis, a SLNB is omitted and an ALND is performed directly. Approximately 15 to $20 \%$ of all patients can be spared from SLNB with this approach ${ }^{15}$. To summarize, axillary US is only able to select half of the patients with node positive.

Several studies suggested recently that the detection and surgical treatment of every single lymph node metastasis is unnecessary. First of all, it is known that the SLNB has a false- negative rate of $9.8 \%{ }^{16}$, which does not compromise loco-regional control or survival $^{19,20}$. Because of the ACOSOG-Z0011 study, the indication to perform a CALND after one or two positive SLN seemed even debatable ${ }^{17,18}$. In this study, the SLN had to contain 1 or 2 metastases, identified with haematoxylin-eosin (H\&E) staining. No immunohistochemical staining (IHC) was used to detect micro metastases or isolated tumor cells (ITC) as they were considered not to be of additional value. In patients treated with CALND, $27.4 \%$ had additional positive nodes removed besides the SLN metastases. In the "wait and see" group no increase of regional recurrence was observed after at least 5 years of follow-up. Between both groups no significant differences in disease free and overall survival was reported ${ }^{5}$.

Furthermore, the threshold to administer adjuvant systemic therapy based on primary tumor characteristics alone has decreased. Besides, the indication is influenced by patient- and tumor related factors, especially after the introduction of different multigene assays.

In conclusion, the detection and treatment of every single lymph node metastasis in patients with breast cancer is not as crucial as previously assumed. Omitting the ALND and even SLNB in a large group of patients with breast cancer could be considered if a non-invasive imaging tool can accurately identify node-negative disease, resulting in a significant reduction of morbidity.

A meta-analysis of positron emission tomography-computer tomography (PET-CT) for the assessment of axillary lymph node metastases in patients with breast cancer reported a sensitivity of only $63 \%$, yet a specificity of $94 \%{ }^{6}$, so PET-CT is not sufficiently accurate in predicting the patients with NO status. The pooled data analysis of 5 studies using USPIO-enhanced MRI showed a mean sensitivity and specificity of $98 \%$ and $96 \%$ respectively, however the USPIO contrast is not approved by the Food and Drug Administration and therefore not available ${ }^{6}$.

The diagnostic performance of gadofosveset enhanced MRI seems superior to other low-weighted gadolinium enhanced MRI lymph node studies. The earlier observed pooled sensitivity and specificity were $88 \%$ and $73 \%{ }^{6}$ for low weigthed gadolinium versus $87 \%$ and $96 \%$ for gadofosveset MRI respectively. In addition the NPV of gadofosveset enhanced MRI (97\%) exceeds the NPV of $91 \%$ in other gadolinium 
enhanced MRI studies. This suggests that gadofosveset enhanced MRI is a better predictor of patient with NO status, compared to low-weighted gadolinium.

False-negative errors with gadofosveset-enhanced MRI occurred mostly in nodes that have micrometastases; however, the clinical relevance of detecting these lesions is questionable. The higher diagnostic performance in detecting patients with NO status using gadofosveset could probably be declared by the special parameters used during the assessment of the lymph nodes, that is, the visual assessment of nodal contrast enhancement and the presence of a 'nodal relief'.

In this study we excluded patients treated with neoadjuvant chemotherapy or hormonal therapy. In current daily practice, there is no consensus about the timing of nodal staging in patients with breast cancer in case of neo-adjuvant therapy. Some institutes perform the SNLB before to neo-adjuvant therapy, others afterwards. A meta-analysis showed that the pathologic status of the SLN after neoadjuvant therapy in patients with clinically node negative nodes accurately predicts the nodal status of the axillary lymph nodes, with a false-negative rate of $7.4 \%{ }^{19}$. This false negative rate is comparable to the false-negative rate in patients undergoing a standard SLNB in patients with early stage breast cancer ${ }^{16}$. Staging and re-staging after neo-adjuvant therapy with gadofosveset enhanced MRI in patients with breast cancer might be an accurate non-invasive diagnostic tool, with the possibility to omit of the SLNB and its morbidity.

Axillary MRI could be performed complementary to breast MRI, which is, nowadays, frequently used for pre-therapeutic staging of breast cancer, (according to Europan Society of Breast Imaging guidelines) because it might affects patients management ${ }^{20,21}$. Although many different MR sequences can be applied in breast imaging ${ }^{22-24}$, current breast MRI protocols in general consist of pre and post contrast imaging, with a post contrast acquisition time of 7 to 10 minutes. Considering the time-management after contrast administration, a combined breast and axilla MRI protocol is feasible. An earlier study in patients with rectal cancer showed a peak enhancement at 17 minutes after gadofosveset administration ${ }^{7}$. In contrast, the peak enhancement in our study was depicted from 11 until 21 minutes after gadofosveset administration. Therefore, reducing the time after contrast administration will shorten the total MRI acquisition time. No evidence regarding the assessment of primary tumors in patients with breast cancer with gadofosveset is available. Furthermore, axillary MRI is challenging since respiratory motion artifacts from the adjacent thoracic wall exist, and discrimination between lymph nodes and adjacent vessels is difficult. However, Baltzer at al. showed that technically axillary staging can be combined with conventional breast MRI examination using a dedicated whole body scanner ${ }^{25}$. Further studies should investigate the accuracy of nodal staging with gadofosveset in a combined breast and axilla MRI scan protocol. 
This feasibility study has its limitations: the low number of included patients, and a total of only 21 macrometastatic lymph nodes. This is reflected in the wide confidence intervals of the calculated sensitivity and PPV. Therefore, the results need to be confirmed in a larger study. The reporting radiologist was blinded for all clinical data of the included patients, although there was a high a-priori chance for detecting lymph node metastasis due to the eligibility for inclusion. This bias might be reflected in the high false positive rate ( 2 patients out of 10 ) in the patient-to-patient analysis in this study.

In conclusion, this study concerning gadofosveset-enhanced MRI of axillary lymph nodes in patients with breast cancer shows promising results. Nevertheless, these results need to be confirmed in a further study. If axillary MRI is as accurate as current diagnostic interventions, this will reduce the number of radiologic and surgical investigations. The need for fewer investigations will make the nodal staging workup more patient friendly (because the morbidity of the total treatment will decrease), more efficient, and cost-effective. 


\section{References}

1. Jemal A, Bray F, Center MM, Ferlay J, Ward E, Forman D. Global cancer statistics. CA Cancer J Clin 2011;61:69-90.

2. Liu CQ, Guo Y, Shi JY, Sheng Y. Late morbidity associated with a tumour-negative sentinel lymph node biopsy in primary breast cancer patients: a systematic review. Eur J Cancer 2009;45:1560-8.

3. Schulze T, Mucke J, Markwardt J, Schlag PM, Bembenek A. Long-term morbidity of patients with early breast cancer after sentinel lymph node biopsy compared to axillary lymph node dissection. J Surg Oncol 2006;93:109-19.

4. Fisher B, Jeong JH, Anderson S, Bryant J, Fisher ER, Wolmark N. Twenty-five-year follow-up of a randomized trial comparing radical mastectomy, total mastectomy, and total mastectomy followed by irradiation. N Engl J Med 2002;347:567-75.

5. Giuliano AE, Hunt KK, Ballman KV, Beitsch PD, Whitworth PW, Blumencranz PW, Leitch AM, Saha S, McCall LM, Morrow M. Axillary dissection vs no axillary dissection in women with invasive breast cancer and sentinel node metastasis: a randomized clinical trial. JAMA 2011;305:569-75.

6. Cooper KL, Meng Y, Harnan S, Ward SE, Fitzgerald P, Papaioannou D, Wyld L, Ingram C, Wilkinson ID, Lorenz E. Positron emission tomography (PET) and magnetic resonance imaging (MRI) for the assessment of axillary lymph node metastases in early breast cancer: systematic review and economic evaluation. Health Technol Assess 2011;15:iii-iv, 1-134.

7. Lahaye GLB, Engelen SME, Voth M, Leiner T, Lambregts DMJ, Beets-Tan RGH. Gadovosfeset Trisodium (Vasovist) Enhanced MR Lymphnode detection: Initial Observations. The Open Magnetic Resonance Journal 2009;2:28-32.

8. Lambregts DM, Beets GL, Maas M, Kessels AG, Bakers FC, Cappendijk VC, Engelen SM, Lahaye MJ, de Bruïne AP, Lammering G, Leiner $T$, Verwoerd JL, Wildberger JE, Beets-Tan RG. Accuracy of gadofosveset-enhanced MRI for nodal staging and restaging in rectal cancer. Ann Surg 2011;253: 5395.

9. Herborn CU, Lauenstein TC, Vogt FM, Lauffer RB, Debatin JF, Ruehm SG. Interstitial MR lymphography with MS-325: characterization of normal and tumor-invaded lymph nodes in a rabbit model. AJR Am J Roentgenol 2002;179:1567-72.

10. Lambregts DM, Heijnen LA, Maas M, Rutten IJ, Martens MH, Backes WH, Riedl RG, Bakers FC, Cappendijk VC, Beets GL, Beets-Tan RG. Gadofosveset-enhanced MRI for the assessment of rectal cancer lymph nodes: predictive criteria. Abdom Imaging 2013;38:720-7.

11. Siesling S, van Dijck JA, Visser O, Coebergh JW; Working Group of The Netherlands Cancer Registry. Trends in incidence of and mortality from cancer in The Netherlands in the period 1989-1998. Eur J Cancer 2003;39:2521-30.

12. Nori J, Vanzi E, Bazzocchi M, Bufalini FN, Distante V, Branconi F, Susini T. Role of axillary ultrasound examination in the selection of breast cancer patients for sentinel node biopsy. Am J Surg 2007;193:1620.

13. Pamilo M, Soiva M, Lavast EM. Real-time ultrasound, axillary mammography, and clinical examination in the detection of axillary lymph node metastases in breast cancer patients. J Ultrasound Med 1989;8:115-20.

14. Valente SA, Levine GM, Silverstein MJ, Rayhanabad JA, Weng-Grumley JG, Ji L, Holmes DR, Sposto R, Sener SF. Accuracy of Predicting Axillary Lymph Node Positivity by Physical Examination, Mammography, Ultrasonography, and Magnetic Resonance Imaging. Ann Surg Oncol 2012;19:1825-30.

15. Alvarez S, Añorbe E, Alcorta P, López F, Alonso I, Cortés J. Role of sonography in the diagnosis of axillary lymph node metastases in breast cancer: a systematic review. AJR Am J Roentgenol 2006;186:1342-8.

16. Krag DN, Anderson SJ, Julian TB, Brown AM, Harlow SP, Ashikaga T, Weaver DL, Miller BJ, Jalovec LM, Frazier TG, Noyes RD, Robidoux A, Scarth HM, Mammolito DM, McCready DR, Mamounas EP, Costantino JP, Wolmark N; National Surgical Adjuvant Breast and Bowel Project. Technical outcomes of sentinel-lymph-node resection and conventional axillary-lymph-node dissection in patients with clinically node-negative breast cancer: results from the NSABP B-32 randomised phase III trial. Lancet Oncol 2007;8:881-8. 
17. Caudle AS, Hunt KK, Kuerer HM, Meric-Bernstam F, Lucci A, Bedrosian I, Babiera GV, Hwang RF, Ross MI, Feig BW, Hoffman K, Litton JK, Sahin AA, Yang W, Hortobagyi GN, Buchholz TA, Mittendorf EA. Multidisciplinary considerations in the implementation of the findings from the American College of Surgeons Oncology Group (ACOSOG) Z0011 study: a practice-changing trial. Ann Surg Oncol. 2011;18:2407-12.

18. Galimberti V, Botteri E, Chifu C, Gentilini O, Luini A, Intra M, Baratella P, Sargenti M, Zurrida S, Veronesi P, Rotmensz N, Viale G, Sonzogni A, Colleoni M, Veronesi U. Can we avoid axillary dissection in the micrometastatic sentinel node in breast cancer? Breast Cancer Res Treat 2012;131:819-25.

19. Tan VK, Goh BK, Fook-Chong S, Khin LW, Wong WK, Yong WS. The feasibility and accuracy of sentinel lymph node biopsy in clinically node-negative patients after neoadjuvant chemotherapy for breast cancer--a systematic review and meta-analysis. J Surg Oncol 2011;104:97-103.

20. Mann RM, Kuhl CK, Kinkel K, Boetes C. Breast MRI: guidelines from the European Society of Breast Imaging. Eur Radiol 2008;18:1307-18.

21. Pediconi F, Miglio E, Telesca M, Luciani ML, Kirchin MA, Passariello R, Catalano C. Effect of preoperative breast magnetic resonance imaging on surgical decision making and cancer recurrence rates. Invest Radiol 2012;47:128-35.

22. Ei Khouli RH, Jacobs MA, Mezban SD, Huang P, Kamel IR, Macura KJ, Bluemke DA. Diffusion-weighted imaging improves the diagnostic accuracy of conventional 3.0-T breast MR imaging. Radiology 2010;256:64-73.

23. Eyal E, Shapiro-Feinberg M, Furman-Haran E, Grobgeld D, Golan T, Itzchak Y, Catane R, Papa M, Degani H. Parametric diffusion tensor imaging of the breast. Invest Radiol 2012;47:284-91.

24. Mizukoshi W, Kozawa E, Inoue K, Saito N, Nishi N, Saeki T, Kimura F. (1)H MR spectroscopy with external reference solution at $1.5 \mathrm{~T}$ for differentiating malignant and benign breast lesions: comparison using qualitative and quantitative approaches. Eur Radiol 2013;23:75-83.

25. Baltzer PA, Dietzel M, Burmeister HP, Zoubi R, Gajda M, Camara O, Kaiser WA. Application of MR mammography beyond local staging: is there a potential to accurately assess axillary lymph nodes? evaluation of an extended protocol in an initial prospective study. AJR Am J Roentgenol 2011;196:W641-7. 


\section{Chapter}

Noninvasive nodal restaging in clinically node positive breast cancer patients after neoadjuvant systemic therapy: a systematic review

RJ. Schipper

M. Moossdorff

R.G.H. Beets-Tan

M.L. Smidt

M.B.I. Lobbes

Eur J Radiol 2015;84:41-7 


\section{Abstract}

\section{Objective}

To provide a systematic review of studies comparing the diagnostic performance of noninvasive techniques and axillary lymph node dissection in the identification of initially node positive patients with pathological complete response of axillary lymph nodes to neoadjuvant systemic therapy.

\section{Methods}

PubMed and Embase databases were searched until May $21^{\text {st }}, 2014$. First, duplicate studies were eliminated. Next, study abstracts were read by two readers to assess eligibility. Studies were selected based on predefined inclusion criteria. Of these, data extraction was performed by two readers independently.

\section{Results}

Of the 987 abstracts that were considered for inclusion, four were eligible for final analysis, which included a total of 572 patients. The diagnostic performance of clinical examination, axillary ultrasound, breast MRI, whole body ${ }^{18}$ F-FDG PET-CT, and a prediction model to identify patients with pathological complete response were investigated. Studies were often limited by small sample size. Furthermore, systemic therapy regimens and definitions of clinical and pathological complete response were variable, refraining further pooling of data. The reported positive predictive value of different techniques to identify patients with axillary pathological complete response after neoadjuvant systemic therapy varied between $40-100 \%$.

\section{Conclusion}

At present, there is no accurate noninvasive restaging technique able to identify patients with complete axillary response after neoadjuvant systemic therapy. 


\section{Introduction}

Breast cancer patients are increasingly treated with neoadjuvant rather than adjuvant systemic therapy ${ }^{1}$. One of the objectives of this approach is downsizing of the primary tumour, enabling breast conservation therapy where mastectomy might be indicated initially ${ }^{2,3}$. Thus, treatment of the breast is tailored to tumour response to neoadjuvant systemic therapy. In contrast, treatment of axillary lymph nodes is mostly based on nodal status at initial diagnosis, regardless of the lymph node response to neoadjuvant systemic therapy.

Assessment of nodal status at initial diagnosis consists of clinical examination, often followed by axillary ultrasound and tissue sampling of suspicious lymph nodes. If an axillary lymph node metastasis is confirmed by histopathology, an axillary lymph node dissection (ALND) is usually indicated, regardless of the response to neoadjuvant systemic therapy. Examination of these ALND specimens showed that after neoadjuvant systemic therapy pathological complete response (pCR) of axillary lymph node metastases is achieved in $20-42 \%{ }^{4-7}$. Since axillary PCR is a strong predictor of disease-free survival ${ }^{8}$, an ALND might be safely omitted in these patients. At present, no studies have confirmed this theory.

Omitting ALND could prevent important short and long-term side effects, such as lymph oedema, limited range of motion of the shoulder, and numbness of the upper $\operatorname{arm}^{9,10}$. These complications are reported up to $45 \%$ of breast cancer survivors ${ }^{11}$. To prevent these complications, the post neoadjuvant systemic therapy sentinel lymph node biopsy was studied. Although this approach seems appropriate in clinically node negative patients undergoing neoadjuvant systemic therapy ${ }^{12,13}$, its accuracy is considered insufficient in clinically node positive patients ${ }^{14}$. Two prospective trials reported a false negative rate of $12.6-14.2 \%$ of this approach ${ }^{13,14}$. A noninvasive technique would be preferable, as it would strongly reduce morbidity by omitting the need for any invasive procedure ${ }^{15}$.

The aim of this study was to provide a systematic review of studies comparing the accuracy of noninvasive techniques to ALND for identifying axillary pCR in initially node positive patients receiving neoadjuvant systemic therapy.

\section{Materials and methods}

For this systematic review, Embase and PubMed (including Medline) databases were searched until May $21^{\text {st }}, 2014$. We used free text and MeSH (Medical Subject Headings) terms for breast cancer, diagnostic performance, lymph nodes, neoadjuvant systemic 
therapy, and a variety of noninvasive diagnostic techniques. Full search strategies are provided in Appendix 5.1.

Prior to the database search, exclusion criteria were determined to avoid selection bias. Exclusion criteria were 1) studies not about breast cancer, 2) or not assessing nodal status, 3) no use of neoadjuvant systemic therapy, 4) or not using a noninvasive modality, 5) case reports, technical notes, comments on earlier publications, editorials, conference proceedings, reviews and meta-analysis, 6) or not using ALND as gold standard, and 7) or in which clinically node positive status was not confirmed by pathology.

First, duplicates were identified and excluded. Next, abstracts were read by two independent readers to assess eligibility. Of all potentially eligible articles, the paper's full text was read. Thereafter, the same two readers in consensus solved discrepancies. Finally, the two readers in consensus searched references of selected articles manually for additional publications. Quality assessment of included articles was performed by using the QUADAS2 tool ${ }^{16}$.

From selected studies, two independent readers using a predefined data extraction form extracted data. The following parameters were extracted: author, year of publication, sample size, age, breast cancer tumour and receptor subtypes, tumour stage, neoadjuvant systemic therapy regimens, diagnostic restaging technique, timing of restaging procedure, and definition and incidence of pathologic complete axillary response. The numbers of true positive, true negative, false positive and false negative cases were extracted and new $2 \times 2$ contingency tables were constructed for each restaging technique separately. Based on these tables, sensitivity, specificity, PPV and negative predictive values (NPV) were re-calculated with corresponding 95\% confidence intervals $(95 \% \mathrm{Cl})$. If complete axillary response was observed, this event was considered as positive, while incomplete response was registered as negative. Thus, the ability of a diagnostic tool to detect patients with axillary PCR is reflected by sensitivity and positive predictive value (PPV) of the diagnostic tool.

\section{Results}

\section{Search strategy and study selection}

In the primary literature search, 1,172 publications were identified. After removing 185 duplicates, 987 potentially eligible articles remained. After reading the abstracts, another 954 studies were excluded, leaving 33 studies of which the full text was read. In this stage, another 29 studies were excluded (see flowchart in Figure 5.1). Four studies were eligible for final analysis in this systematic review ${ }^{5,17-19}$. 
Records identified through Embase database search $(n=878)$
Additional records identified through PubMed database search $(n=294)$

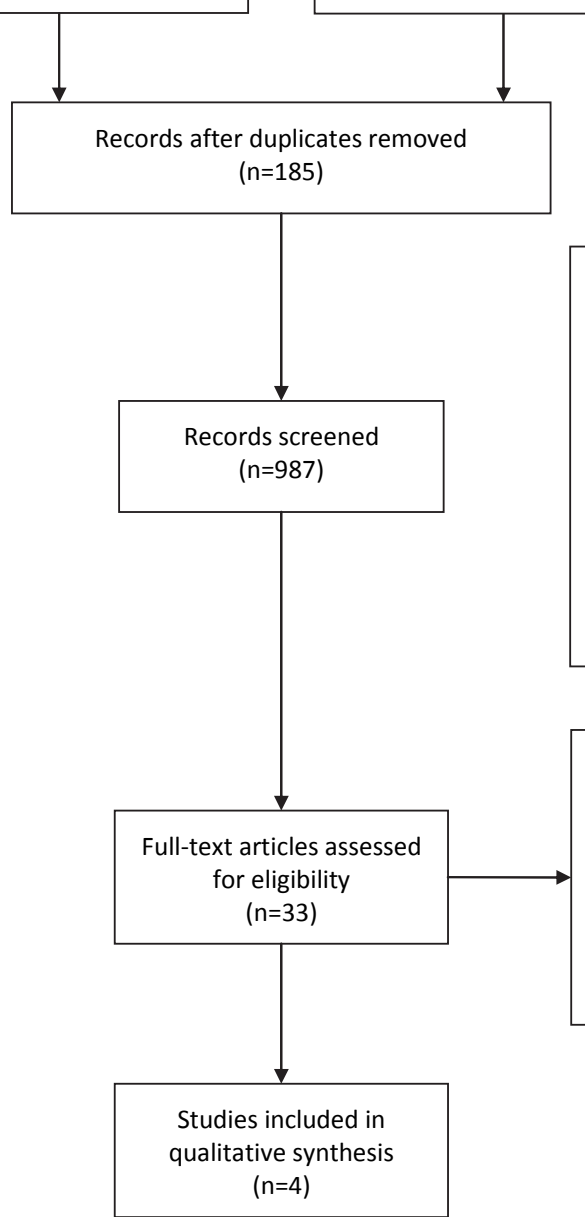

Records excluded ( $n=954)$

Not about breast cancer $(n=318)$

Not assessing axillary nodal status $(n=300)$

No neoadjuvant systemic therapy $(n=174)$

No noninvasive diagnostic tool $(n=56)$

No original diagnostic study $(n=85)$

Reference test other than ALND $(n=5)$

No subset of clinically node positive

patients based on ultrasound guided

tissue sampling $(n=6)$

No separate data on axillary pathologic

nodal status $(n=1)$

Duplicate dataset $(n=9)$

Full-text articles excluded, with reasons $(n=29)$

Not assessing axillary nodal status $(n=4)$

No subset of clinically node positive

patients based on ultrasound guided

tissue sampling $(n=20)$

No separate data on axillary pathologic

nodal status $(n=5)$

Figure 5.1 Flowchart of study selection process according to the Preferred Reporting Items for Systematic Reviews and Meta-Analyses (PRISMA).

\section{General study characteristics}

A total of 572 patients participated in these four studies $(32,80,169$, and 251 per study respectively). All patients had cytological or histological proven axillary metastasis at initial diagnosis. The diagnostic performance of clinical examination to identify patients with axillary complete response to neoadjuvant systemic therapy was reported in one study $^{17}$. Two studies investigated axillary ultrasound ${ }^{17,18}$, one breast magnetic resonance imaging $(\mathrm{MRI})^{18}$ and two studies whole body $\left[{ }^{18} \mathrm{~F}\right]$-fluorodeoxyglucose 
positron emission tomography and computed tomography $\left({ }^{18} \mathrm{~F}-\mathrm{FDG} \text { PET-CT }\right)^{5,18}$. One study describes a model for predicting PCR based on baseline patient and tumour characteristics $^{19}$. Neoadjuvant systemic therapy regimens varied not only between studies, but also even within studies. The percentage of initially node positive patients who achieved axillary pCR varied from $21.9 \%$ to $38.5 \%$. A detailed overview of all study characteristics is provided in Table 5.1. The included studies showed low risk of bias, although some risk of bias was present due to patient selection and unclear use of the reference test. Additionally, there were concerns regarding applicability of the results, as shown in Table 5.2 .

Pooling of the data was not performed for several reasons: First, the number of included studies per diagnostic tool was limited. Second, between the studies that did investigate the same diagnostic tool, heterogeneity was caused by varying cut-off points of the used tests (e.g. PET-positive lymph node, suspicious lymph node on ultrasound). Third, a wide variety of systemic therapy regimens were used. Forth, inclusion criteria between studies were heterogeneous regarding tumour type and T-stage. The low number of studies precluded us from performing funnel plot analysis to rule out publication bias.

\section{Diagnostic performance of each nodal restaging technique}

\section{Clinical examination}

Arimappamagan et al. ${ }^{17}$ investigated the use of clinical examination to evaluate axillary response to neoadjuvant systemic therapy in 32 patients. Their definition of complete clinical axillary response was described as "the absence of any palpable lymph node in the axilla". Pathological complete response was defined as "the disappearance of all known disease, and additional sections were taken in lymph nodes showing complete response". Based on the new $2 \times 2$ contingency table (see Table 5.3), the reported sensitivity, specificity, PPV and NPV for detecting patients with axillary pathological complete response were $86 \%, 64 \%, 40 \%$, and $94 \%$, respectively (Table 5.4 ).

\section{Axillary ultrasound}

Arimappamagan et al. ${ }^{17}$ also investigated the accuracy of axillary ultrasound in the same group of patients as mentioned above. It is unclear whether the radiologist was blinded for the results of physical examination of the axillary lymph nodes after neoadjuvant systemic therapy. Axillary ultrasound for nodal restating was performed after finishing all cycles of neoadjuvant systemic therapy. Sonographically complete axillary response was defined as "the absence of any detectable lymph node in the axilla" after neoadjuvant systemic therapy. The diagnostic performance of ultrasound outperformed clinical examination with sensitivity, specificity, PPV and NPV of $86 \%$, $100 \%, 100 \%$, and $96 \%$, respectively (Table 5.4 ). 


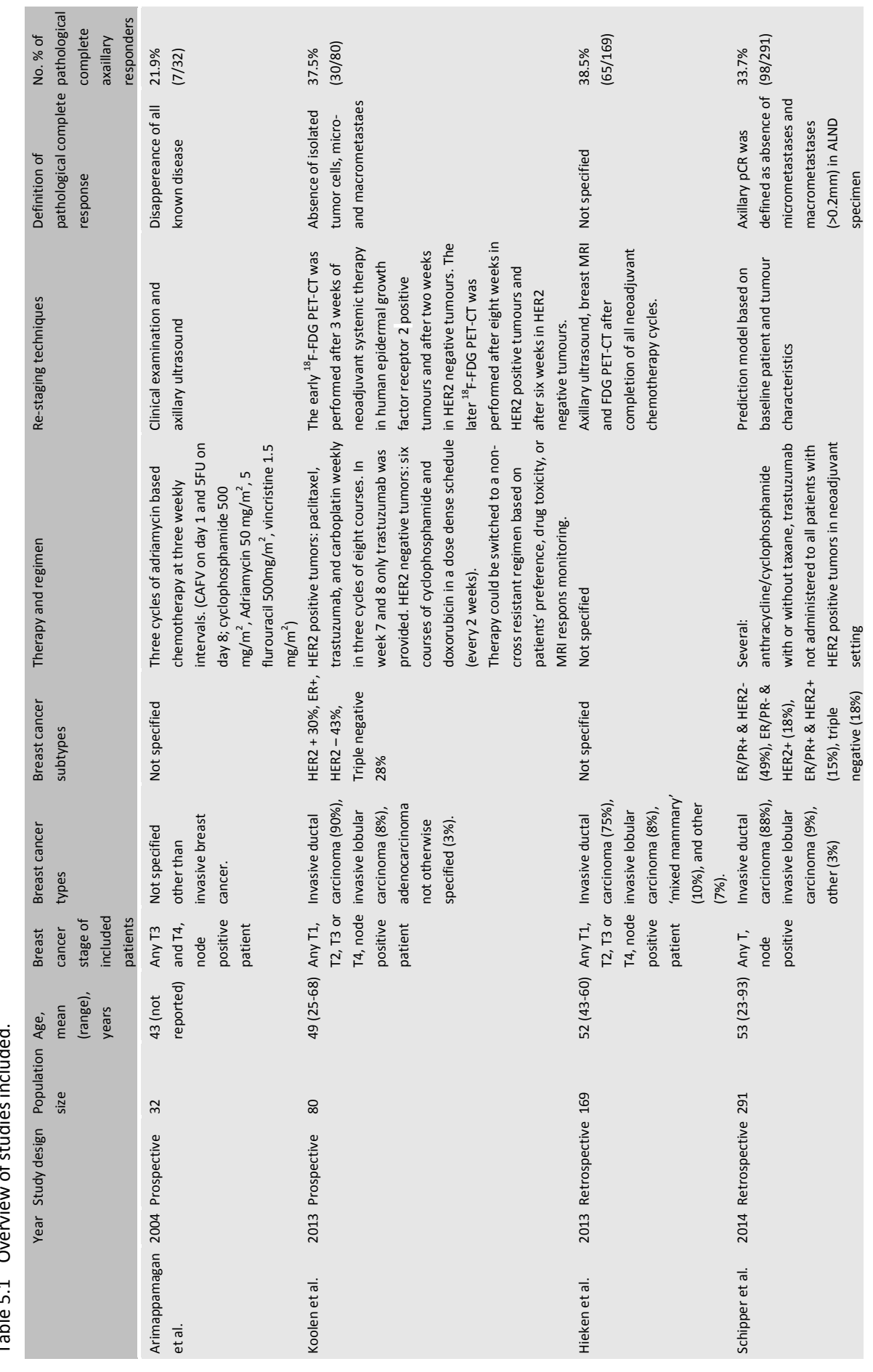


Table 5.2 Quality assessment of the included studies based on QUADAS2.

\begin{tabular}{|c|c|c|c|c|c|c|c|}
\hline & \multicolumn{4}{|c|}{ Risk of bias } & \multicolumn{3}{|c|}{ Applicability concerns } \\
\hline & $\begin{array}{l}\text { Patient } \\
\text { selection }\end{array}$ & Index test & $\begin{array}{c}\text { Reference } \\
\text { test }\end{array}$ & $\begin{array}{l}\text { Flow of } \\
\text { patients }\end{array}$ & $\begin{array}{l}\text { Patient } \\
\text { selection }\end{array}$ & Index test & $\begin{array}{c}\text { Reference } \\
\text { test }\end{array}$ \\
\hline Arimappamagan et al. & ;: & (:) & $?$ & ;) & ;: & (:) & ;) \\
\hline Koolen et al. & ;) & ;) & ;) & (:) & ;) & ;) & ;) \\
\hline Hieken et al. & 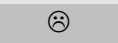 & ;) & ;) & (:) & $?$ & (:) & (:) \\
\hline Schipper et al. & : : & ;) & (:) & (:) & (:) & (:) & ;) \\
\hline
\end{tabular}

;) low risk, : high risk, ? unclear risk

Table 5.3 Absolute numbers from $2 \times 2$ contingency tables for included studies and each restaging tool separate.

\begin{tabular}{llccccc} 
Study & Restaging tool & $\begin{array}{c}\text { True } \\
\text { positive }\end{array}$ & $\begin{array}{c}\text { False } \\
\text { positive }\end{array}$ & $\begin{array}{c}\text { False } \\
\text { negative }\end{array}$ & $\begin{array}{c}\text { True } \\
\text { negative }\end{array}$ & $\begin{array}{c}\text { Total } \\
\text { no. pts. }\end{array}$ \\
\hline Arimappamagan et al. & Clinical examination & 6 & 9 & 1 & 16 & 32 \\
& Axillary Ultrasound & 6 & 0 & 1 & 25 & 32 \\
Koolen et al. & FDG PET-CT & 12 & 2 & 13 & 35 & 62 \\
Hieken et al. & Axillary ultrasound & 25 & 19 & 18 & 44 & 106 \\
& MRI & 17 & 23 & 12 & 36 & 88 \\
Schipper et al. & FDG PET-CT & 11 & 7 & 2 & 12 & 32 \\
Prediction model & 42 & 23 & 56 & 170 & 291
\end{tabular}

Hieken et al. ${ }^{18}$ investigated the diagnostic performance of axillary ultrasound in a subset of their included patients. They included 169 fine needle aspiration confirmed node positive breast cancer patients, of whom 106 underwent axillary ultrasound after completion of all cycles of neoadjuvant systemic therapy. The definition of axillary pathological complete response and histopathological evaluation of the ALND specimen were not specified. Sonographically complete axillary response was considered present if "the appearance of the lymph nodes had normalized". This resulted in sensitivity, specificity, PPV and NPV of $58 \%, 70 \%, 57 \%$, and $71 \%$, respectively (Table 5.4 ).

Table 5.4 On overview on the diagnostic performance of each restaging technique per restaging tool for predicting axillary pathological complete response to neoadjuvant systemic treatment.

$\begin{array}{llcccc}\text { Restaging tool } & \text { Study } & \text { Sensitivity } & \text { Specificity } & \text { PPV } & \text { NPV } \\ \text { Clinical examination } & \text { Arimappamagan et al. } & 86(42-99) & 64(43-81) & 40(17-67) & 94(69-100) \\ \text { Axillary Ultrasound } & \text { Arimappamagan et al. } & 86(42-99) & 100(83-100) & 100(52-100) & 96(79-99) \\ & \text { Hieken et al. } & 58(42-73) & 70(57-80) & 57(41-71) & 71(58-82) \\ \text { MRI } & \text { Hieken et al. } & 59(39-76) & 61(47-73) & 43(27-59) & 75(60-86) \\ \text { FDG PET-CT } & \text { Koolen et al. } & 48(28-68) & 95(80-99) & 86(56-97) & 73(58-84) \\ & \text { Hieken et al. } & 85(54-97) & 63(39-83) & 61(36-82) & 86(56-97 \\ \text { Nomogram } & \text { Schipper et al. } & 43(33-53) & 88(82-92) & 65(52-76) & 75(69-81)\end{array}$

PPV: positive predictive value; NPV: negative predictive value. $95 \%$ confidence intervals between parentheses. PPV and NPV at prevalence of axillary pathological complete response as reported in original publication. 
MRI

In the study of Hieken et al. ${ }^{18} 88$ out of 169 patients underwent a post neoadjuvant systemic therapy breast MRI on which the axillary region was adequate visualized. Breast MRI was performed with a 1.5T magnet, using a dedicated breast coil. Again the definition of complete clinical response was defined as "normalized appearance of the lymph node(s)". With the use of a breast MRI, sensitivity, specificity, PPV and NPV was reported to be $59 \%, 61 \%, 43 \%$, and $75 \%$ (Table 5.4 ).

\section{${ }^{18}$ F-FDG PET-CT}

Hieken et al. ${ }^{18}$ reported also on the use of ${ }^{18}$ F-FDG PET-CT for the purpose of axillary restaging after neoadjuvant systemic therapy. In 32 patients, a post neoadjuvant systemic therapy ${ }^{18}$ F-FDG PET-CT was available. Definition of complete axillary response was "normalization of the lymph node, and there have to be at least resolution of FDG activity of the lymph node(s)". The use of "F-FDG PET-CT showed a sensitivity, specificity, PPV, and NPV of $85 \%, 63 \%, 61 \%$ and $86 \%$, respectively (Table 5.4 ).

Koolen et al. ${ }^{5}$ evaluated the value of sequential ${ }^{18}$ F-FDG PET-CT early in the courses of neoadjuvant systemic therapy for axillary response evaluation. ${ }^{18} \mathrm{~F}$-FDG PET-CTs were performed at baseline $(n=80)$, in 62 patients an early ${ }^{18}$ F-FDG PET-CT was performed and in 77 patients later during neoadjuvant systemic therapy. The early ${ }^{18} \mathrm{~F}$-FDG PET-CT was performed after 3 weeks of neoadjuvant systemic therapy in human epidermal growth factor receptor 2 (HER2) positive tumours and after two weeks in HER2 negative tumours. The later ${ }^{18}$ F-FDG PET-CT was performed after eight weeks in HER2 positive tumours and after six weeks in HER2 negative tumours. On every scan, the SUV $_{\max }$ of the node with highest SUV $\mathrm{Vax}_{\max }$ was calculated. This was not necessarily the same node on each scan. Thereafter, the relative decrease in $S_{U} V_{\max }$ was calculated with regard to the baseline scan and a ROC-curve was constructed with histopathology as gold standard. With an AUC of 0.52 , the baseline SUV $_{\max }$ was not associated with axillary pCR. In contrast, a decrease of $S V_{\text {max }}$ with $\geq 60 \%$ on the first scan during neoadjuvant systemic therapy had a sensitivity, specificity, PPV and NPV of $48 \%, 95 \%$, $86 \%$ and $73 \%$ for complete axillary response (Table 5.4). According to the authors, an ${ }^{18}$ F-FDG PET-CT later during neoadjuvant systemic therapy did not improve the diagnostic performance.

\section{Prediction model}

One study described development of a model to predict axillary PCR based on patient and tumour characteristics at initial diagnosis ${ }^{19}$. The model was based on retrospectively collected data of 291 patients and includes the following parameters: age, T-stage, histologic subtype, receptor status (ER/PR/HER2), whether the treatment regimen was taxane-based, and whether trastuzumab was administered neoadjuvant (for HER2 positive tumours). Completing the nomogram will result in a nomogram- 
score, which corresponds with specific sensitivity and specificity for detection of axillary pCR. Although accuracy was reasonable (area under the ROC curve of $0.77,95 \% \mathrm{Cl}$ $0.71-0.81)$, the model lacked specificity $(88 \%)$ resulting in a positive predictive value of $65 \%$ (Table 5.4).

\section{Discussion}

This systematic review provides an overview of studies comparing the diagnostic performance of noninvasive techniques to ALND, to identify axillary PCR to neoadjuvant systemic therapy, in biopsy proven node positive breast cancer patients. Four studies were identified, evaluating the diagnostic performance of clinical examination, axillary ultrasound, breast MRI, whole body ${ }^{18}$ F-FDG PET-CT, and a prediction model. These studies varied widely regarding type of neoadjuvant systemic therapy regimens and definitions of clinical and pathological complete response. The reported PPV of the different techniques to identify patients with axillary pCR varied between $40-100 \%$.

An accurate noninvasive restaging technique for identifying patients with axillary complete response would be extremely beneficial for breast cancer care. The five-year survival of node positive breast cancer patients has improved over the last decades to $84.4 \%{ }^{20}$, while morbidity that is associated with an ALND and radiotherapy, which are performed routinely in clinically node positive breast cancer patients, can compromise their quality of life ${ }^{21-23}$. During the last decade, the administration of systemic therapy in node positive patients has shifted from the adjuvant to the neoadjuvant setting. As a result, $20 \%$ to $42 \%$ of initially node positive patients achieve pCR of the axillary lymph nodes $^{4-8}$. Consequently, ALND might be omitted in these patients. Furthermore, patients with axillary pCR are unlikely to develop a regional recurrence ${ }^{24}$; suggesting that even axillary and periclavicular radiotherapy might be avoided in these patients. Accurate (noninvasive) selection of patients with complete axillary response could have important consequences for breast cancer survivors.

Clinical examination of the axillary lymph nodes is not feasible for identifying patients with axillary PCR, due to its low sensitivity and PPV of $86 \%$ and $40 \%$, respectively. It is not expected that the diagnostic performance of clinical examination can be improved, since clinical examination for nodal staging in the primary surgery setting also suffers from many false negative findings. This also applies to the prediction model. At the most optimal specificity, the estimated PPV is only $65 \%{ }^{19}$. Furthermore, the model detects only half of patients with $\mathrm{pCR}$, thereby missing many patients who might be candidates for less extensive surgery.

In contrast, axillary ultrasound after neoadjuvant systemic therapy seems very accurate with a PPV of $100 \%$, when using the definition "absence of any lymph node on ultrasound" to predict axillary pathological complete response ${ }^{17}$. In contrast, Hieken et 
al. used the definition that the lymph nodes must be 'normalized', resulting in a PPV of $58 \%$. The definition of Arimappamagan raises questions; it is our experience that when performing axillary ultrasound, lymph nodes are detectable in every patient. This suggests that the definition as proposed by Arimappamagan et al. may not be easily applicable and reproducible, thereby introducing inter-observer variation. These are problems of which axillary ultrasound also suffers in the primary staging setting ${ }^{25}$. Nonetheless, the reported sensitivity of this approach of $86 \%$ was high. Therefore, results of this technique should be studied in larger populations with multiple radiologists.

With respect to breast MRI and ${ }^{18}$ F-FDG PET-CT, current evidence shows that both techniques lack sufficient sensitivity (48\%-85\%) and specificity (61\%-95\%) to act as a substitute for invasive staging techniques. Restaging axillary nodes with MRI or ${ }^{18} \mathrm{~F}$-FDG PET-CT is difficult, which is not surprising. Even in the primary staging setting both techniques have shortcomings in ruling out the presence of metastases. It seems that sequential MRI or ${ }^{18}$ F-FDG PET-CT are not helpful for identifying patients with axillary pCR. One of the reasons of this phenomenon might be the absence of clear evaluation criteria. This is for example reflected in the definition of axillary complete clinical response on MRI as used by Hieken; "normalized appearance of the lymph node(s)". However, it is unclear what the maximum size of a normalized lymph node is and which morphologic criteria are applied. Furthermore, MRI of the axillary region is technically challenging, since respiratory motion causes artefacts from the adjacent thoracic wall. Also, discrimination between lymph nodes and adjacent vascular structures is difficult, which can lead to misinterpretation of the true lymph node status ${ }^{26}$.

With regard to ${ }^{18}$ F-FDG PET-CT, a pitfall is its limited capacity of detecting small residual tumour deposits, probably because the limited metabolic activity of microscopic residual. This problem has been described earlier in studies using ${ }^{18}$ F-FDG PET-CT for primary lymph node staging ${ }^{27}$. From these studies, it is known that ${ }^{18}$ F-FDG PET-CT suffers from many false negative results. The study of Koolen et al. ${ }^{5}$ showed that this problem can partly be overcome by monitoring the relative decrease in SUV $V_{\max }$, rather than evaluating the absolute absence of FDG uptake after all chemotherapy cycles, as performed in the study of Hieken et al. ${ }^{18}$. However, only half of the patients with complete axillary response could be identified using the approach of Koolen et al..

With all this in mind, confirmation studies using MRI or ${ }^{18}$ F-FDG PET-CT are not justified at this moment. First, diagnostic performance must be improved. Opportunities for improvement can be identified in several steps of the process.

\section{Suggested improvements for future studies regarding MRI and ${ }^{18}$ F-FDG PET-CT}

With respect to breast MRI, as investigated by Hieken et al., one of the reasons of its moderate diagnostic performance for nodal restaging is due it suffers from low axillary signal to noise ratio. This leads to inadequate visualization of the entire axilla. The use 
of additional coils to the standard breast MRI coil or using a dedicated whole body MRI, as showed by Baltzer et al., can overcome this ${ }^{28}$. An alternative is to perform a separate dedicated axillary MRI without simultaneous breast $\mathrm{MRI}^{29}$. Although this requires an additional MRI examination, it does have the advantage of facilitating the use of a dedicated lymph node contrast agent, for example gadofosveset or ultrasmall superparamagnetic iron oxide (USPIO). Both contrast agents showed their added value for primary lymph node staging in breast cancer patients ${ }^{27,29}$.

Regarding ${ }^{18}$ F-FDG PET-CT, the studies discussed in this review have already assessed several moments for response evaluation during the course of neoadjuvant therapy without satisfactory results. It questionable if investigating alternative evaluation moments could further improve the diagnostic performance of ${ }^{18} \mathrm{~F}$-FDG PET-CT. In that light, potentially, technical improvements of the PET detector can facilitate depiction of small foci of metabolic activity ${ }^{30}$. One could design dedicated axillary PET detectors, in line with the development of dedicated breast PET devices. Results of dedicated breast PET devices are promising compared to conventional whole body PET-CT devices ${ }^{31}$.

Furthermore, PET tracers other than ${ }^{18} \mathrm{~F}$-FDG may provide better results ${ }^{32}$. Promising results are reported with the use of $3^{\prime}$-deoxy-3'-fluorothymide (FLT). FLT was found to correlate well with the degree of cell proliferation ${ }^{33}$ and could therefore may be used as parameter for treatment effectiveness during neoadjuvant systemic therapy ${ }^{34,35}$.

Finally, breast cancer is a heterogeneous disease, which is only partly expressed with difference in hormonal and HER2 receptor status. It has been reported that the diagnostic performance of MRI and ${ }^{18}$ F-FDG PET-CT depends on breast cancer subtypes, as well as on the type of systemic therapy. Therefore, large multicentre studies with substantial numbers of patients are recommended to assess the diagnostic performance of each diagnostic tool per subtype for nodal restaging after various subtypes of systemic therapy.

This systemic review has several important limitations. First, as in every systemic review, there is the problem of publication bias. Studies with unfavourable results are published less frequently. With this in mind, one should realize that the diagnostic performance of different restaging techniques might be overestimated. Second, the number of studies discussed is limited. Many studies were excluded for various reasons, the vast majority because patients were staged as node positive by clinical examination or imaging only. In our opinion, histopathological confirmation of the lymph node status is an essential part in these kinds of studies, since it is known that clinical examination and imaging suffers from false positive cases ${ }^{25,36,37}$. Including of these studies would result in overestimation of the true diagnostic performance.

In conclusion, no accurate noninvasive technique currently exists for restaging of the axillary lymph nodes in initially clinically node positive breast cancer patients after neoadjuvant systemic therapy. Further studies should confirm the promising diagnostic 
performance of axillary ultrasound for nodal restaging. With respect to MRI and ${ }^{18}$ F-FDG PET-CT, improvements of the techniques itself and optimization of outcome parameters must be considered and studied in larger study populations. 


\section{Appendix 5.1}

\section{Embase search strategy}

1. breast.mp. [mp=title, abstract, subject headings, heading word, drug trade name, original title, device manufacturer, drug manufacturer, device trade name, keyword]

2. mamma.mp. [mp=title, abstract, subject headings, heading word, drug trade name, original title, device manufacturer, drug manufacturer, device trade name, keyword]

3. 1 or 2

4. cancer.mp. [mp=title, abstract, subject headings, heading word, drug trade name, original title, device manufacturer, drug manufacturer, device trade name, keyword]

5. carcinoma.mp. [mp=title, abstract, subject headings, heading word, drug trade name, original title, device manufacturer, drug manufacturer, device trade name, keyword]

6. neoplasm.mp. [mp=title, abstract, subject headings, heading word, drug trade name, original title, device manufacturer, drug manufacturer, device trade name, keyword]

7. malignancy.mp. [mp=title, abstract, subject headings, heading word, drug trade name, original title, device manufacturer, drug manufacturer, device trade name, keyword]

8. adenocarcinoma.mp. [mp=title, abstract, subject headings, heading word, drug trade name, original title, device manufacturer, drug manufacturer, device trade name, keyword]

9. 4 or 5 or 6 or 7 or 8

10. 3 and 9

11. PET.mp. [mp=title, abstract, subject headings, heading word, drug trade name, original title, device manufacturer, drug manufacturer, device trade name, keyword]

12. Positron-Emission Tomography.mp. [ $\mathrm{mp}=$ title, abstract, subject headings, heading word, drug trade name, original title, device manufacturer, drug manufacturer, device trade name, keyword]

13. Positron emission tomography.mp. [ $\mathrm{mp}=$ title, abstract, subject headings, heading word, drug trade name, original title, device manufacturer, drug manufacturer, device trade name, keyword]

14. Magnetic resonance imaging.mp. [ $\mathrm{mp}=$ title, abstract, subject headings, heading word, drug trade name, original title, device manufacturer, drug manufacturer, device trade name, keyword] 
15. MRI.mp. [mp=title, abstract, subject headings, heading word, drug trade name, original title, device manufacturer, drug manufacturer, device trade name, keyword]

16. magnetic resonance. $\mathrm{mp}$. [ $\mathrm{mp}=$ title, abstract, subject headings, heading word, drug trade name, original title, device manufacturer, drug manufacturer, device trade name, keyword]

17. MR.mp. [mp=title, abstract, subject headings, heading word, drug trade name, original title, device manufacturer, drug manufacturer, device trade name, keyword]

18. ultrasonography.mp. [mp=title, abstract, subject headings, heading word, drug trade name, original title, device manufacturer, drug manufacturer, device trade name, keyword]

19. ultrasound.mp. [mp=title, abstract, subject headings, heading word, drug trade name, original title, device manufacturer, drug manufacturer, device trade name, keyword]

20. physical examination.mp. [mp=title, abstract, subject headings, heading word, drug trade name, original title, device manufacturer, drug manufacturer, device trade name, keyword]

21. clinical examination.mp. [ $\mathrm{mp}=$ title, abstract, subject headings, heading word, drug trade name, original title, device manufacturer, drug manufacturer, device trade name, keyword]

22. palpation.mp. [mp=title, abstract, subject headings, heading word, drug trade name, original title, device manufacturer, drug manufacturer, device trade name, keyword]

23. nomogram.mp. [mp=title, abstract, subject headings, heading word, drug trade name, original title, device manufacturer, drug manufacturer, device trade name, keyword]

24. decision support technique.mp. [mp=title, abstract, subject headings, heading word, drug trade name, original title, device manufacturer, drug manufacturer, device trade name, keyword]

25. prediction model.mp. [ $\mathrm{mp}=$ title, abstract, subject headings, heading word, drug trade name, original title, device manufacturer, drug manufacturer, device trade name, keyword]

26. CT.mp. [mp=title, abstract, subject headings, heading word, drug trade name, original title, device manufacturer, drug manufacturer, device trade name, keyword]

27. computed tomography.mp. [mp=title, abstract, subject headings, heading word, drug trade name, original title, device manufacturer, drug manufacturer, device trade name, keyword] 
28. 18F-FDG PET-CT.mp. [mp=title, abstract, subject headings, heading word, drug trade name, original title, device manufacturer, drug manufacturer, device trade name, keyword]

29. 11 or 12 or 13 or 14 or 15 or 16 or 17 or 18 or 19 or 20 or 21 or 22 or 23 or 24 or 25 or 26 or 27 or 28

30. sensitivity.mp. [ $\mathrm{mp}=$ title, abstract, subject headings, heading word, drug trade name, original title, device manufacturer, drug manufacturer, device trade name, keyword]

31. specificity.mp. [mp=title, abstract, subject headings, heading word, drug trade name, original title, device manufacturer, drug manufacturer, device trade name, keyword]

32. predictive value.mp. [ $\mathrm{mp}=$ title, abstract, subject headings, heading word, drug trade name, original title, device manufacturer, drug manufacturer, device trade name, keyword]

33. positive predictive value.mp. [ $\mathrm{mp}=$ title, abstract, subject headings, heading word, drug trade name, original title, device manufacturer, drug manufacturer, device trade name, keyword]

34. negative predictive value.mp. [ $\mathrm{mp}=$ title, abstract, subject headings, heading word, drug trade name, original title, device manufacturer, drug manufacturer, device trade name, keyword]

35. likelihood ratio.mp. [ $\mathrm{mp}=$ title, abstract, subject headings, heading word, drug trade name, original title, device manufacturer, drug manufacturer, device trade name, keyword]

36. PPV.mp. [mp=title, abstract, subject headings, heading word, drug trade name, original title, device manufacturer, drug manufacturer, device trade name, keyword]

37. NPV.mp. [mp=title, abstract, subject headings, heading word, drug trade name, original title, device manufacturer, drug manufacturer, device trade name, keyword]

38. diagnosis.mp. [mp=title, abstract, subject headings, heading word, drug trade name, original title, device manufacturer, drug manufacturer, device trade name, keyword]

39. 30 or 31 or 32 or 33 or 34 or 35 or 36 or 37 or 38

40. axilla*.mp. [mp=title, abstract, subject headings, heading word, drug trade name, original title, device manufacturer, drug manufacturer, device trade name, keyword]

41. lymph*.mp. [mp=title, abstract, subject headings, heading word, drug trade name, original title, device manufacturer, drug manufacturer, device trade name, keyword]

42. 40 or 41 
43. node.mp. [mp=title, abstract, subject headings, heading word, drug trade name, original title, device manufacturer, drug manufacturer, device trade name, keyword]

44. metastasis.mp. [mp=title, abstract, subject headings, heading word, drug trade name, original title, device manufacturer, drug manufacturer, device trade name, keyword]

45. nodes.mp. [mp=title, abstract, subject headings, heading word, drug trade name, original title, device manufacturer, drug manufacturer, device trade name, keyword]

46. 43 or 44 or 45

47. 42 and 46

48. neoadjuvant.mp. [ $\mathrm{mp}=$ title, abstract, subject headings, heading word, drug trade name, original title, device manufacturer, drug manufacturer, device trade name, keyword]

49. primary.mp. [mp=title, abstract, subject headings, heading word, drug trade name, original title, device manufacturer, drug manufacturer, device trade name, keyword]

50. preoperative.mp. [ $\mathrm{mp}=$ title, abstract, subject headings, heading word, drug trade name, original title, device manufacturer, drug manufacturer, device trade name, keyword]

51. 48 or 49 or 50

52. therapy.mp. [mp=title, abstract, subject headings, heading word, drug trade name, original title, device manufacturer, drug manufacturer, device trade name, keyword]

53. immunotherapy.mp. [mp=title, abstract, subject headings, heading word, drug trade name, original title, device manufacturer, drug manufacturer, device trade name, keyword]

54. chemotherapy.mp. [mp=title, abstract, subject headings, heading word, drug trade name, original title, device manufacturer, drug manufacturer, device trade name, keyword]

55. systemic.mp. [mp=title, abstract, subject headings, heading word, drug trade name, original title, device manufacturer, drug manufacturer, device trade name, keyword]

56. 52 or 53 or 54 or 55

57. 51 and 56

58. 10 and 29 and 39 and 47 and 57

\section{Pubmed search strategy}

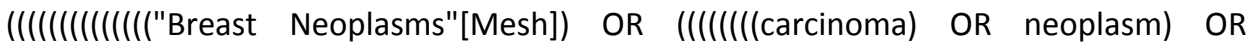
neoplasms) OR malignancy) OR adenocarcinoma) OR cancer)) AND breast)) OR ((mamma) AND ((((((carcinoma) OR neoplasm) OR neoplasms) OR malignancy) OR 
adenocarcinoma) OR cancer $))))))$ AND ((((((((("Positron-Emission Tomography"[Mesh] OR "positron emission tomography" OR PET)) OR ("Magnetic Resonance Imaging"[Mesh] OR "magnetic resonance" OR MRI)) OR ("Ultrasonography"[Mesh] OR ultrasonography OR ultrasound)) OR ("Physical Examination"[Mesh] OR "physical examination" OR "clinical examination" OR "palpation")) OR (("Nomograms"[Mesh]) OR "Decision Support Techniques"[Mesh] OR "prediction model" OR nomogram)) OR (("Tomography, X-Ray Computed"[Mesh]) OR "Positron-Emission Tomography and Computed Tomography"[Mesh] OR CT OR 18F-FDG PET-CT))))) AND (((((((((("Sensitivity and Specificity"[Mesh]) OR "Predictive Value of Tests"[Mesh])) OR ((sensitivity) OR specificity)) $O R$ "negative predictive value") OR "positive predictive value") $O R$ "likelihood ratio") OR "diagnosis"))))) AND (((((((("Lymph Nodes"[Mesh]) OR "Lymphatic Vessels"[Mesh]) OR "Lymphatic Metastasis"[Mesh]) OR "Lymphatic System"[Mesh])) OR (("Axilla"[Mesh]) OR axilla*)))))) $\quad$ AND (("Neoadjuvant Therapy"[Mesh]) OR (((((neoadjuvant) OR preoperative) OR primary)) AND ("Antineoplastic Agents"[Mesh] OR chemotherapy OR immunotherapy OR systemic therapy))) 


\section{References}

1. Kaufmann M, von Minckwitz G, Mamounas EP, Cameron D, Carey LA, Cristofanilli M, Denkert C, Eiermann W, Gnant M, Harris JR, Karn T, Liedtke C, Mauri D, Rouzier R, Ruckhaeberle E, Semiglazov V, Symmans WF, Tutt A, Pusztai L. Recommendations from an international consensus conference on the current status and future of neoadjuvant systemic therapy in primary breast cancer. Ann Surg Oncol 2012;19:1508-16.

2. Fisher B, Bryant J, Wolmark N, Mamounas E, Brown A, Fisher ER, Wickerham DL, Begovic M, DeCillis A, Robidoux A, Margolese RG, Cruz AB Jr, Hoehn JL, Lees AW, Dimitrov NV, Bear HD. Effect of preoperative chemotherapy on the outcome of women with operable breast cancer. J Clin Oncol 1998;16:2672-85.

3. van der Hage JA, van de Velde CJ, Julien JP, Tubiana-Hulin M, Vandervelden C, Duchateau L. Preoperative chemotherapy in primary operable breast cancer: results from the European Organization for Research and Treatment of Cancer trial 10902. J Clin Oncol 2001;19:4224-37.

4. Alvarado R, Yi M, Le-Petross H, Gilcrease M, Mittendorf EA, Bedrosian I, Hwang RF, Caudle AS, Babiera GV, Akins JS, Kuerer HM, Hunt KK. The role for sentinel lymph node dissection after neoadjuvant chemotherapy in patients who present with node-positive breast cancer. Ann Surg Oncol 2012;19: 3177-84.

5. Koolen BB, Valdés Olmos RA, Wesseling J, Vogel WV, Vincent AD, Gilhuijs KG, Rodenhuis S, Rutgers EJ, Vrancken Peeters MJ. Early assessment of axillary response with (1)(8)F-FDG PET/CT during neoadjuvant chemotherapy in stage II-III breast cancer: implications for surgical management of the axilla. Ann Surg Oncol 2013;20:2227-35.

6. Rouzier R, Extra JM, Klijanienko J, Falcou MC, Asselain B, Vincent-Salomon A, Vielh P, Bourstyn E. Incidence and prognostic significance of complete axillary downstaging after primary chemotherapy in breast cancer patients with T1 to T3 tumors and cytologically proven axillary metastatic lymph nodes. J Clin Oncol 2002;20:1304-10.

7. Straver ME, Rutgers EJ, Russell NS, Oldenburg HS, Rodenhuis S, Wesseling J, Vincent A, Peeters MT. Towards rational axillary treatment in relation to neoadjuvant therapy in breast cancer. Eur J Cancer 2009;45:2284-92.

8. von Minckwitz G, Untch M, Blohmer JU, Costa SD, Eidtmann H, Fasching PA, Gerber B, Eiermann W, Hilfrich J, Huober J, Jackisch C, Kaufmann M, Konecny GE, Denkert C, Nekljudova V, Mehta K, Loibl S. Definition and impact of pathologic complete response on prognosis after neoadjuvant chemotherapy in various intrinsic breast cancer subtypes. J Clin Oncol 2012;30:1796-804.

9. Ashikaga T, Krag DN, Land SR, Julian TB, Anderson SJ, Brown AM, Skelly JM, Harlow SP, Weaver DL, Mamounas EP, Costantino JP, Wolmark N; National Surgical Adjuvant Breast, Bowel Project. Morbidity results from the NSABP B-32 trial comparing sentinel lymph node dissection versus axillary dissection. J Surg Oncol 2010;102:111-8.

10. Liu CQ, Guo Y, Shi JY, Sheng Y. Late morbidity associated with a tumour-negative sentinel lymph node biopsy in primary breast cancer patients: a systematic review. Eur J Cancer 2009;45:1560-8.

11. Ahmed RL, Prizment A, Lazovich D, Schmitz KH, Folsom AR. Lymphedema and quality of life in breast cancer survivors: the lowa Women's Health Study. J Clin Oncol 2008;26:5689-96.

12. Fontein DB, van de Water W, Mieog JS, Liefers GJ, van de Velde CJ., Timing of the sentinel lymph node biopsy in breast cancer patients receiving neoadjuvant therapy - recommendations for clinical guidance. Eur J Surg Oncol 2013;39:417-24.

13. Kuehn T, Bauerfeind I, Fehm T, Fleige B, Hausschild M, Helms G, Lebeau A, Liedtke C, von Minckwitz G, Nekljudova V, Schmatloch S, Schrenk P, Staebler A, Untch M. Sentinel-lymph-node biopsy in patients with breast cancer before and after neoadjuvant chemotherapy (SENTINA): a prospective, multicentre cohort study. Lancet Oncol 2013;14:609-18.

14. Boughey JC, Suman VJ, Mittendorf EA, Ahrendt GM, Wilke LG, Taback B, Leitch AM, Kuerer HM, Bowling M, Flippo-Morton TS, Byrd DR, Ollila DW, Julian TB, McLaughlin SA, McCall L, Symmans WF, Le-Petross HT, Haffty BG, Buchholz TA, Nelson H, Hunt KK; Alliance for Clinical Trials in Oncology. Sentinel lymph node surgery after neoadjuvant chemotherapy in patients with node-positive breast cancer: the ACOSOG Z1071 (Alliance) clinical trial. JAMA 2013;310:1455-61. 
15. DiSipio T, Rye S, Newman B, Hayes S. Incidence of unilateral arm lymphoedema after breast cancer: a systematic review and meta-analysis. Lancet Oncol 2013;14:500-15.

16. Whiting PF, Rutjes AW, Westwood ME, Mallett S, Deeks JJ, Reitsma JB, Leeflang MM, Sterne JA, Bossuyt PM; QUADAS-2 Group. QUADAS-2: a revised tool for the quality assessment of diagnostic accuracy studies. Ann Intern Med 2011;155:529-36.

17. Arimappamagan A, Kadambari D, Srinivasan K, Krishnan R, Elangovan S, Reddy KS. Complete axillary conversion after neoadjuvant chemotherapy in locally advanced breast cancer: a step towards conserving axilla? Indian J Cancer 2004;41:13-7.

18. Hieken TJ, Boughey JC, Jones KN, Shah SS, Glazebrook KN. Imaging response and residual metastatic axillary lymph node disease after neoadjuvant chemotherapy for primary breast cancer. Ann Surg Oncol 2013;20:3199-204.

19. Schipper RJ, Moossdorff M, Nelemans PJ, Nieuwenhuijzen GA, de Vries B, Strobbe LJ, Roumen RM, van den Berkmortel F, Tjan-Heijnen VC, Beets-Tan RG, Lobbes MB, Smidt ML. A model to predict pathologic complete response of axillary lymph nodes to neoadjuvant chemo(immuno)therapy in patients with clinically node-positive breast cancer. Clin Breast Cancer 2014;14:315-22.

20. N, H., N. AM, K. M, G. J, N. N, A. SF, K. CL, Y. M, R. J, T. Z, C. H, M. A, L. DR, C. HS, F. EJ, and C.K. (eds). SEER Cancer Statistics Review, 1975-2010. 2013 April 2013 [cited 2014 02-05]; Available from: http://seer.cancer.gov/csr/1975_2010/.

21. Heiney SP, McWayne J, Cunningham JE, Hazlett L, Parrish RS, Bryant LH, Vitoc C, Jansen K. Quality of life and lymphedema following breast cancer. Lymphology 2007;40:177-84.

22. Pyszel A, Malyszczak K, Pyszel K, Andrzejak R, Szuba A. Disability, psychological distress and quality of life in breast cancer survivors with arm lymphedema. Lymphology 2006;39:185-92.

23. Shih YC, Xu Y, Cormier JN, Giordano S, Ridner SH, Buchholz TA, Perkins GH, Elting LS. Incidence, treatment costs, and complications of lymphedema after breast cancer among women of working age: a 2-year follow-up study. J Clin Oncol 2009;27:2007-14.

24. Mamounas EP, Anderson SJ, Dignam JJ, Bear HD, Julian TB, Geyer CE Jr, Taghian A, Wickerham DL, Wolmark N. Predictors of locoregional recurrence after neoadjuvant chemotherapy: results from combined analysis of National Surgical Adjuvant Breast and Bowel Project B-18 and B-27. J Clin Oncol 2012;30:3960-6.

25. Diepstraten SC, Sever AR, Buckens CF, Veldhuis WB, van Dalen T, van den Bosch MA, Mali WP, Verkooijen HM. Value of preoperative ultrasound-guided axillary lymph node biopsy for preventing completion axillary lymph node dissection in breast cancer: a systematic review and meta-analysis. Ann Surg Oncol 2014;21:51-9.

26. Heijnen LA, Lambregts DM, Martens MH, Maas M, Bakers FC, Cappendijk VC, Oliveira P, Lammering G, Riedl RG, Beets GL, Beets-Tan RG. Performance of gadofosveset-enhanced MRI for staging rectal cancer nodes: can the initial promising results be reproduced? Eur Radiol 2014;24:371-9.

27. Cooper KL, Meng Y, Harnan S, Ward SE, Fitzgerald P, Papaioannou D, Wyld L, Ingram C, Wilkinson ID, Lorenz E. Positron emission tomography (PET) and magnetic resonance imaging (MRI) for the assessment of axillary lymph node metastases in early breast cancer: systematic review and economic evaluation. Health Technol Assess 2011;15:1-134.

28. Baltzer PA, Dietzel M, Burmeister HP, Zoubi R, Gajda M, Camara O, Kaiser WA. Application of MR mammography beyond local staging: is there a potential to accurately assess axillary lymph nodes? evaluation of an extended protocol in an initial prospective study. AJR Am J Roentgenol 2011;196: W641-7.

29. Schipper RJ, Smidt ML, van Roozendaal LM, Castro CJ, de Vries B, Heuts EM, Keymeulen KB, Wildberger $\mathrm{JE}$, Lobbes MB, Beets-Tan RG. Noninvasive nodal staging in patients with breast cancer using gadofosveset-enhanced magnetic resonance imaging: a feasibility study. Invest Radiol 2013;48: 134-9.

30. El Fakhri G, Surti S, Trott CM, Scheuermann J, Karp JS. Improvement in lesion detection with wholebody oncologic time-of-flight PET. J Nucl Med 2011;52:347-53.

31. Koolen BB, Vogel WV, Vrancken Peeters MJ, Loo CE, Rutgers EJ, Valdés Olmos RA. Molecular Imaging in Breast Cancer: From Whole-Body PET/CT to Dedicated Breast PET. J Oncol 2012;2012:438647.

32. Linden HM, Dehdashti F. Novel methods and tracers for breast cancer imaging. Semin Nucl Med 2013;43:324-9. 
33. Bading JR, Shields AF. Imaging of cell proliferation: status and prospects. J Nucl Med 2008;49 Suppl 2:64S-80S.

34. Kenny LM, Al-Nahhas A, Aboagye EO. Novel PET biomarkers for breast cancer imaging. Nucl Med Commun 2011;32:333-5.

35. Pio BS, Park CK, Pietras R, Hsueh WA, Satyamurthy N, Pegram MD, Czernin J, Phelps ME, Silverman DH. Usefulness of 3'-[F-18]fluoro-3'-deoxythymidine with positron emission tomography in predicting breast cancer response to therapy. Mol Imaging Biol 2006;8:36-42.

36. Alvarez S, Añorbe E, Alcorta P, López F, Alonso I, Cortés J. Role of sonography in the diagnosis of axillary lymph node metastases in breast cancer: a systematic review. AJR Am J Roentgenol 2006; 186:1342-8.

37. Valente SA, Levine GM, Silverstein MJ, Rayhanabad JA, Weng-Grumley JG, Ji L, Holmes DR, Sposto R, Sener SF. Accuracy of predicting axillary lymph node positivity by physical examination, mammography, ultrasonography, and magnetic resonance imaging. Ann Surg Oncol 2012;19:1825-30. 


\section{Chapter}

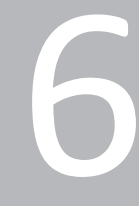

A model to predict pathologic complete response of axillary lymph nodes to neoadjuvant chemo(-immuno)therapy in patients with clinically node positive breast cancer

RJ. Schipper

M. Moossdorff

P.J. Nelemans

G.A.P. Nieuwenhuijzen

B. de Vries

L.J.A. Strobbe

R.M.H. Roumen

F. van den Berkmortel

V.C.G. Tjan-Heijnen

R.G.H. Beets-Tan

M.B.I. Lobbes

M.L. Smidt

Clin Breast Cancer 2014;14:315-22 


\section{Abstract}

\section{Introduction}

Between $20-42 \%$ of patients with clinically node positive breast cancer achieve pathologic complete response of axillary lymph nodes after neoadjuvant chemo(-immuno)therapy. Hypothetically, axillary lymph node dissection may be safely omitted in these patients. This study aims to develop a model for predicting axillary pathologic complete response in these patients.

\section{Methods}

We retrospectively identified clinically node positive breast cancer patients who were treated with neoadjuvant chemo(-immuno)therapy and axillary lymph node dissection between 2005 and 2012 in five hospitals. Patient and tumor characteristics, neoadjuvant chemo(-immuno)therapy regimen and pathology reports were extracted. Binary logistic regression analysis was used to predict axillary pathologic complete response, using the following variables: age, tumor stage and type, hormone receptor and HER2 status, and administration of taxane and trastuzumab. The model was internally validated by bootstrap resampling. The overall performance of the model was assessed by the Brier score and the discriminative performance by Receiver Operator Characteristic (ROC) analysis.

\section{Results}

A model was developed based on 291 patients and internally validated with a scaled Brier score of 0.14 . The area under the ROC curve of this model was 0.77 [ $95 \% \mathrm{Cl} 0.71-0.82$ ]. At a cut-off value of predicted probability $\geq 0.50$, the model demonstrated a specificity of $88 \%$, sensitivity of $43 \%$, positive predictive value of $65 \%$, and negative predictive value of $75 \%$.

\section{Conclusion}

This prediction model shows reasonable accuracy to predict axillary PCR. However, omitting axillary treatment based on the nomogram score solely is not justified. Further research is warranted to non-invasively identify patients with axillary pathological complete response. 


\section{Introduction}

Breast cancer patients who are clinically node positive at diagnosis increasingly undergo chemo(-immuno)therapy in the neoadjuvant rather than adjuvant setting.

Pathological complete response (pCR) of lymph node metastases is achieved in $20-42 \%$ of clinically node positive patients after neoadjuvant chemo(-immuno)therapy and is a strong predictor of disease-free survival ${ }^{1-5}$. Patients who achieve axillary PCR may be safely treated without axillary lymph node dissection (ALND). To identify patients in whom ALND may be omitted, a non-invasive method approximating the accuracy of ALND is needed to evaluate axillary lymph node response to neoadjuvant chemo(-immuno)therapy.

In the Netherlands, the standard method for assessing nodal status in breast cancer patients is clinical examination, followed by axillary ultrasound and cytology or histology of suspicious lymph nodes. If axillary lymph node metastasis is confirmed at diagnosis, the standard treatment includes ALND in addition to surgical treatment of the primary tumor, regardless of the axillary nodal response to neoadjuvant chemo(-immuno)therapy. Although the idea has never never confirmed in a randomized trial, ALND is unlikely to improve locoregional control and survival in patients with axillary PCR. Identification of axillary PCR and subsequent omission of ALND could prevent short and long-term side effects of this procedure, such as lymph edema, limited range of motion of the shoulder, and numbness of the upper arm ${ }^{6,7}$.

Until now, ALND is the gold standard to ascertain that $\mathrm{PCR}$ is achieved. In order to identify initially node positive patients with axillary $\mathrm{PCR}$ after neoadjuvant chemo(-immuno)therapy, several non-invasive and invasive techniques were investigated previously.

The diagnostic accuracy of non-invasive techniques such as physical examination, axillary ultrasound and PET-CT determining axillary $\mathrm{pCR}$ after neoadjuvant chemo(-immuno)therapy was evaluated ${ }^{1-2}$. However, none proved sufficiently accurate (Table 6.1).

Table 6.1 Diagnostic value of different modalities for predicting axillary pCR after neoadjuvant treatment.

$\begin{array}{lccc} & \text { Sensitivity } & \text { Specificity } & \text { PPV }^{1} \\ \text { Physical examinationa [1] } & 90 \% & 14 \% & 42 \%{ }^{\#} \\ \text { Axillary ultrasound [1] } & 60 \% & 58 \% & 51 \%{ }^{\#} \\ \text { PET-CT [12] } & 48 \% & 95 \% & 86 \%^{\wedge}\end{array}$

1: positive predictive value at prevalence of $\mathrm{pCR}$ as reported in original publication. \# prevalence of pCR $42 \%$; $\wedge$ prevalence of pCR $40 \%$

Post-chemo(-immuno)therapy sentinel lymph node biopsy (SLNB) in clinically node positive patients has its limitations as well, namely suboptimal identification rate $(80-94 \%)$ and relatively high false negative rate $(10-20 \%)^{8-11}$. Consequently, some patients could be at risk of developing nodal recurrence if ALND were omitted. 
Furthermore, SLNB is an invasive procedure with possible short and long-term morbidities comparable to ALND, although these occur less frequently ${ }^{12}$. After SLNB, 8\% of patients suffer from lymph edema after a follow-up of three years ${ }^{6}$.

To summarize, no non-invasive techniques that approximates the accuracy of ALND for identifying axillary pathologic complete response in clinically node positive patients after neoadjuvant chemo(-immuno)therapy are currently available. The aim of this study is to develop a prediction model that approximates the accuracy of ALND for identifying clinically node positive patients who achieve axillary $\mathrm{PCR}$ after neoadjuvant chemo(-immuno)therapy.

\section{Materials and methods}

\section{Patients}

This study retrospectively identified clinically node positive breast cancer patients treated with neoadjuvant chemo(-immuno)therapy between 2005 and 2012 in one university hospital and four regional teaching hospitals. Patients were eligible for inclusion if they met the following criteria: 1 ) had clinically node positive breast cancer based on an axillary ultrasound with pathologically confirmed metastasis before neoadjuvant chemo(-immuno)therapy; 2) completed all cycles of planned-dose neoadjuvant chemo(-immuno)therapy; 3) underwent both radical excision of the primary tumor and ALND. Patients were excluded if they had a history of surgery of the ipsilateral axilla. Node positive patients based on SLNB prior to the first dose of neoadjuvant chemo(-immuno)therapy were not included, because it was uncertain whether additional metastatic lymph nodes were present in the axilla during neoadjuvant chemo(-immuno)therapy.

\section{Data extraction}

Data were extracted from patient records regarding date of birth, tumor characteristics (clinical T-stage, based on combination of imaging and physical examination; and histological subtype, estrogen- and progesterone receptor status, and HER2 status, based on core biopsy results), neoadjuvant chemo(-immuno)therapy regimen (type of chemotherapy, administration of trastuzumab), type of surgical treatment of the breast, and postoperative pathological characteristics of the tumor and axillary lymph nodes.

\section{Neoadjuvant treatment regimens}

Neoadjuvant chemo(-immuno)therapy was provided in accordance with the Dutch breast cancer guidelines ${ }^{13-15}$. During the study period, two major changes were implemented. First, a taxane containing regimen was introduced in 2006 (added to 
anthracycline/cyclophosphamide-based chemotherapy). Second, in 2008 trastuzumab was added to neoadjuvant chemotherapy in patients with HER2 positive tumors, whereas in 2005-2008, trastuzumab was given as adjuvant treatment only. As these changes are considered to improve overall survival, it is plausible they also influenced pCR rates ${ }^{16}$. Therefore, patients' chemo(-immuno)therapy regimens were classified into two groups, i.e. with or without a taxane. In addition, we documented if trastuzumab was administered during neoadjuvant treatment.

\section{Surgical treatment}

Surgical treatment was performed in accordance with the Dutch breast cancer guidelines $\left[13^{-15}\right.$. Surgery consisted of resection of the primary tumor (either mastectomy or wide local excision followed by radiation therapy) and performance of a level 1 and 2 ALND.

\section{Pathology}

Core biopsies and surgical specimens were evaluated according to the Dutch breast cancer guidelines $\left[13^{15}\right.$. The initial core biopsy of the primary tumor was evaluated using standard haematoxylin and eosin staining, immunohistochemistry and/or fluorescence or chromogenic in situ hybridization for histological subtype, estrogen and progesterone receptor status (positive if $\geq 10 \%$ expression), and HER2 status. The tumor grade on biopsy was not recorded separately as this was not relevant for the selection of the neoadjuvant treatment regimen ${ }^{17,18}$.

Response to neoadjuvant chemo(-immuno)therapy of the primary tumor and axillary lymph nodes was recorded. In this study, PCR of the primary breast tumor was defined as the absence of invasive tumor cells in the resection specimen (non-invasive breast residuals allowed, as suggested by other authors $\left.{ }^{19,20}\right)$. Axillary $\mathrm{PCR}$ was defined as absence of micro- and macrometastases $(>0.2 \mathrm{~mm})$ in the axillary lymph nodes. Isolated tumor cells were not considered as residual disease, as these do not seem to influence disease-free survival in clinically node positive patients who have undergone neoadjuvant chemo(-immuno)therapy ${ }^{21}$.

\section{Statistical analysis}

Descriptive data are presented as proportions and absolute numbers for categorical data and as means with standard deviations for continuous variables. The development and evaluation of a prediction model was performed in several steps.

1. For development of the prediction model, predefined variables were included in a multivariate binary logistic regression analysis to determine corresponding regression coefficients with $95 \%$ confidence intervals. The predefined variables were: age, tumor stage (cT3 versus CT1-2; cT4 versus cT1-2), tumor type (lobular versus ductal carcinoma; other types versus ductal carcinoma), estrogen and 
progesterone receptor status and HER2 status, type of chemo(-immuno)therapy regimen (taxane versus non-taxane), and administration of trastuzumab as neoadjuvant treatment.

2. The model was internally validated by bootstrap resampling. This is a method in which sampling, model building and evaluation are repeated a large number of times ${ }^{22}$. Bootstrapping adjusts for overoptimism of the original model by providing a shrinkage factor, which is the preferred technique for internal validation according to Steyerberg et al. ${ }^{23}$. Shrinkage of the regression coefficients aims to improve calibration of predictions in future patients ${ }^{24}$.

3. A nomogram based on the internally validated model was constructed to facilitate calculation of predicted probabilities for individual patients and these predicted probabilities are referred to as nomogram scores. A nomogram allows for calculation of a sum score for each patient based on the presence or level of clinical characteristics in a patient and shows the predicted probability of axillary pCR that is associated with a specific sum score. In this way, a nomogram score can be assigned to individual patients.

4. Test performance of nomogram scores was assessed by using several parameters. The Brier score is a measure of agreement between predicted and observed probabilities and ranges from 0 (perfect model) to 1 (worthless model) ${ }^{25}$. The calibration plot visualizes the agreement between observed outcomes and predictions, and the area under the Receiver Operating Characteristic (ROC) curve is a measure for ability of prediction model scores to discriminate between patient with and without axillary PCR. The ROC curve shows the trade-off between sensitivity and specificity at several cut-off values of the nomogram scores.

5. Based on the prevalence of pCR of $34 \%$, post-test probabilities of axillary pCR were calculated at different cut-off values. The positive predictive value (PPV) represents the probability of presence of axillary $\mathrm{PCR}$ if the nomogram score is equal to or exceeds the cut-off value and the negative predictive value (NPV) represents the probability of absence of axillary PCR if the nomogram score is below the cut-off value. The decision to omit ALND requires a high PPV.

R-software was used to perform the statistical analyses; including bootstrapping and drawing of a nomogram to visually represent the model (www.R-project.org, version 2.0; R Foundation for Statistical Computing, Vienna, Austria).

\section{Results}

\section{Patient characteristics}

This study included 291 clinically node positive breast cancer patients who were treated with neoadjuvant chemo(-immuno)therapy. The distribution of patient, tumor 
and treatment characteristics for the total study population and the subgroups with and without $\mathrm{pCR}$ of the axillary lymph nodes are summarized in Table 6.2. Mean age was 53 years (SD 11.4) and mean tumor size was $41 \mathrm{~mm}$ (SD 26.6). Most patients (88\%) were diagnosed with invasive ductal carcinoma. Estrogen receptor was positive in 62.9\%. A HER2 positive tumor was present in 96 (33.0\%) patients, of whom 52 (54.2\%) received trastuzumab in the neoadjuvant setting. All remaining patients with HER2 positive tumors received trastuzumab as adjuvant treatment. In $78.4 \%$ of the patients, the neoadjuvant chemotherapy regimen contained a taxane.

Table 6.2 Patient and tumor characteristics at baseline.

\begin{tabular}{|c|c|c|c|}
\hline & No-axillary pCR & Axillary pCR & All patients \\
\hline Total no. of patients & 193 & 98 & 291 \\
\hline \multicolumn{4}{|l|}{ Age, years } \\
\hline Mean (SD) & $53.6(11.4)$ & $50.9(11.1)$ & 53 \\
\hline Range & $27-93$ & $23-87$ & 23-93 \\
\hline \multicolumn{4}{|l|}{ Clinical tumor category (\%) } \\
\hline сT1 & $19(73.1)$ & $7(26.9)$ & 26 \\
\hline cT2 & $79(68.1)$ & 37 (31.9) & 116 \\
\hline сT3 & $53(62.4)$ & $32(37.6)$ & 85 \\
\hline cT4 & $42(65.6)$ & $22(34.4)$ & 64 \\
\hline \multicolumn{4}{|l|}{ Tumor type (on tumor biopsy) (\%) } \\
\hline Invasive ductal & $167(65.2)$ & $89(34.8)$ & 256 \\
\hline Invasive lobular & $22(81.5)$ & $5(18.5)$ & 27 \\
\hline Other invasive & $4(50.0)$ & $4(50.0)$ & 8 \\
\hline \multicolumn{4}{|l|}{ Neoadjuvant Systemic therapy regimen } \\
\hline Taxane based & $155(68.0)$ & $73(32.0)$ & 228 \\
\hline Non-taxane based & $38(60.3)$ & $25(39.7)$ & 63 \\
\hline \multicolumn{4}{|l|}{ HER2 status } \\
\hline Negative & $147(75.4)$ & $48(24.6)$ & 195 \\
\hline Positive & $46(47.9)$ & $50(52.1)$ & 96 \\
\hline Neoadjuvant trastuzumab & $19(36.5)$ & $33(63.5)$ & 52 \\
\hline Adjuvant trastuzumab & $27(61.4)$ & $17(38.6)$ & 44 \\
\hline \multicolumn{4}{|l|}{ Receptor status (on tumor biopsy) (\%) } \\
\hline Triple negative & $26(50.0)$ & $26(50.0)$ & 52 \\
\hline$E R / P R+H E R 2-$ & $121(84.6)$ & $22(15.4)$ & 143 \\
\hline $\mathrm{ER} / \mathrm{PR}$ - HER2 + & $23(43.4)$ & $30(56.6)$ & 53 \\
\hline $\mathrm{ER} / \mathrm{PR}+\mathrm{HER} 2+$ & $23(53.5)$ & $20(47.5)$ & 43 \\
\hline
\end{tabular}

pCR: pathological complete response; ER: Estrogen Receptor; PR: Progesterone Receptor; HER2: Human Epidermal growth factor Receptor 2.

\section{Incidence of pCR of primary tumor and axillary lymph nodes}

After neoadjuvant chemo(-immuno)therapy, PCR of the axillary lymph nodes was observed in $33.7 \%(98 / 291)$ of the patients and PCR of the breast tumor in $25.8 \%$ (75/291). More specifically, $17.5 \%$ of the patients (51/291) had pCR of both the primary tumor and the axillary lymph nodes. In addition, $8.2 \%(24 / 291)$ had pCR of the primary 
tumor but not of the axillary lymph nodes, and $16.2 \%$ (47/291) had pCR of the axillary lymph nodes alone. Further information on tumor and nodal stage after neoadjuvant chemo(-immuno)therapy is summarized in Table 6.3.

Table 6.3 Pathologic tumor characteristics after neo-adjuvant chemo(-immuno)therapy.

\begin{tabular}{|c|c|c|}
\hline & $\mathrm{N}$ & $\%$ \\
\hline \multicolumn{3}{|l|}{ Pathologic tumor category } \\
\hline урто & 63 & 21.6 \\
\hline ypTis & 12 & 4.1 \\
\hline урT1 & 127 & 43.6 \\
\hline урт2 & 46 & 15.8 \\
\hline урТ3 & 30 & 10.3 \\
\hline урт4 & 7 & 2.4 \\
\hline \multicolumn{3}{|l|}{ Pathologic nodal category } \\
\hline ypNi+ & 6 & 2.1 \\
\hline ypN1mi & 8 & 2.7 \\
\hline ypNO & 92 & 31.6 \\
\hline ypN1 & 111 & 38.1 \\
\hline ypN2 & 56 & 19.2 \\
\hline ypN3 & 18 & 6.2 \\
\hline \multicolumn{3}{|l|}{ Combination tumour and nodal category } \\
\hline Axillary $p C R+/$ Tumor pCR - & 47 & 16.2 \\
\hline Axillary pCR - / Tumor pCR + & 24 & 8.2 \\
\hline Axillary $\mathrm{pCR}+$ / Tumor pCR + & 51 & 17.5 \\
\hline Axillary pCR - / Tumor pCR - & 169 & 58.1 \\
\hline
\end{tabular}

pCR: pathological complete response

\section{Development of the model}

Binary logistic regression analysis was performed including the ten predefined variables available at diagnosis. Patients with HER2 positive tumors who had been treated with both chemotherapy and immunotherapy were more likely to achieve pCR of the axillary lymph nodes (OR $3.93(95 \% \mathrm{Cl} 1.62-12.11)$ ). In contrast, patients with estrogen receptor positive tumors were least likely to achieve axillary nodal pCR $(O R \quad 0.39(95 \% \mathrm{Cl}$ 0.16-0.90). All results of the binary logistic regression analysis are shown in Table 6.4. 
Table 6.4 Results of the binary logistic regression analysis; regression coefficients and odds ratios before and after internal validation by bootstrapping.

\begin{tabular}{|c|c|c|c|}
\hline & $\begin{array}{l}\text { Regression } \\
\text { coefficient }\end{array}$ & $\begin{array}{c}\text { OR } \\
(95 \% \mathrm{Cl})\end{array}$ & $\begin{array}{l}\text { Regression coefficient after } \\
\text { bootstrapping }(n=1000)\end{array}$ \\
\hline Intercept & 1.264 & & 0.934 \\
\hline Age (per year) & -0.028 & $0.97(0.94-0.99)$ & -0.023 \\
\hline \multicolumn{4}{|l|}{ Tumour size category } \\
\hline cT1-2 & & 1.00 & \\
\hline сT3 & 0.544 & $1.72(0.89-3.56)$ & 0.441 \\
\hline cT4 & 0.244 & $1.28(0.55-3.00)$ & 0.198 \\
\hline \multicolumn{4}{|l|}{ Tumor type category } \\
\hline Ductal & & 1.00 & \\
\hline Lobular & -0.052 & $0.95(0.21-2.56)$ & -0.042 \\
\hline Other type & 0.850 & $2.34(0.29-15.93)$ & 0.689 \\
\hline \multicolumn{4}{|l|}{ Estrogen receptor status } \\
\hline ER negative & & 1.00 & \\
\hline ER positive & -0.941 & $0.39(0.16-0.90)$ & -0.762 \\
\hline \multicolumn{4}{|l|}{ Progesterone receptor status } \\
\hline PR negative & & 1.00 & \\
\hline PR positive & -0.324 & $0.72(0.30-1.79)$ & -0.262 \\
\hline \multicolumn{4}{|l|}{ HER2 receptor status } \\
\hline HER2 negative & & 1.00 & \\
\hline HER2 positive & 0.253 & $1.29(0.54-2.73)$ & 0.205 \\
\hline \multicolumn{4}{|c|}{ Trastuzumab containing neoadjuvant therapy regimen* } \\
\hline No & & 1.00 & \\
\hline Yes & 1.368 & $3.93(1.62-12.11)$ & 1.108 \\
\hline \multicolumn{4}{|c|}{ Taxane containing neoadjuvant therapy regimen } \\
\hline No & & 1.00 & \\
\hline Yes & -0.526 & $0.59(0.26-1.37)$ & -0.426 \\
\hline
\end{tabular}

*Only applicable for patients with HER2 positive tumor. The regression coefficient estimates the amount of increase (or decrease, if the sign of the coefficient is negative) in the predicted log odds of axillary pathological complete response holding all other predictors constant. Tumor type was categorized into 'ductal', 'lobular' and 'other type' and was entered as dummy variables with ductal type as reference category. OR: odds ratio; 95\%-CI: 95\% confidence interval; HER2: Human Epidermal growth factor Receptor 2.

\section{Internal validation}

Internal validation by bootstrapping $(n=1000)$ resulted in a shrinkage factor of 0.81 . The regression coefficients from the original model were multiplied by this factor and the adjusted regression coefficients are presented in Table 6.4. A nomogram based on these results is shown in Figure 6.1. 
Points

Age

cT3

cT4

Invasive lobular carcinoma

Histologic subtype

ER status

PR status

HER2 status

Received trastuzumab

Received taxane

Total points

Nomogram score

(predicted probability)

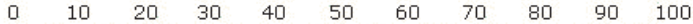

$\begin{array}{llllllllllllllll}95 & 90 & 85 & 80 & 75 & 70 & 65 & 60 & 55 & 50 & 45 & 40 & 35 & 30 & 25 & 20\end{array}$

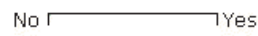

No TYes

Yes $\sqcap$ No

ILC or IDC $\longrightarrow$ Other

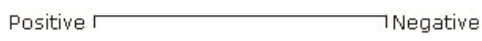

Positive Negative

Negative $P$ Positive

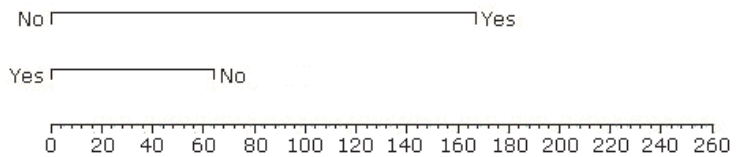

$\begin{array}{lllll}0.05 & 0.1 & 0.125 & 0.5 & 0.75\end{array}$

Figure 6.1 A model to predict the likelihood of achieving axillary pathological complete response to neoadjuvant chemo(-immuno)therapy.

Find the score for each variable for an individual patient on the uppermost rule ("points"). Secondly, add all scores together and find the sum of the scores on the "total points" rule. The corresponding nomogram score (predicted probability) can be found on the lowest rule.

A nomogram score of 0.5 or higher identifies pathological complete response with a specificity of $88 \%$, sensitivity of $43 \%$, positive predictive value (PPV) of $65 \%$, and negative predictive value (NPV) of $75 \%$. Diagnostic performance at different cut-off values of the predicted probability are listed in Table 6.5.

Table 6.5 Values of sensitivity, specificity and predictive values of the nomogram scores at different cutoff values.

\begin{tabular}{|c|c|c|c|c|}
\hline Nomogram score/ Predicted probability & Sensitivity, \% & Specificity, \% & PPV, \% & NPV, \% \\
\hline$\geq 0.20$ & 88 & 48 & 47 & 89 \\
\hline$\geq 0.30$ & 73 & 67 & 53 & 83 \\
\hline$\geq 0.40$ & 57 & 80 & 59 & 78 \\
\hline$\geq 0.50$ & 43 & 88 & 65 & 75 \\
\hline$\geq 0.60$ & 27 & 93 & 67 & 71 \\
\hline$\geq 0.70$ & 17 & 96 & 69 & 69 \\
\hline$\geq 0.80$ & 2 & 98 & 34 & 66 \\
\hline
\end{tabular}

PPV: positive predictive value, NPV: negative predictive value. The predicted probability/nomogram score is a numeric value representing the prediction model score of the individual patient. The nomogram score can be used as a test parameter and a positive test result can be defined as a score that is equal to or exceeds a specific cut-off value. A negative test result is defined as a score below the cut-off value. The PPV and NPV are calculated at the observed prevalence of axillary PCR of 34\%. PPV: positive predictive value; NPV: negative predictive value. 


\section{Performance of the model}

The Brier score was 0.19 , with a scaled (to maximum) Brier score of 0.14 . Figure 6.2 presents the calibration plot of the internally validated model. A ROC curve (Figure 6.3) was constructed based on the nomogram scores. The AUC was 0.77 ( $95 \% \mathrm{Cl} 0.71-0.82$ ).

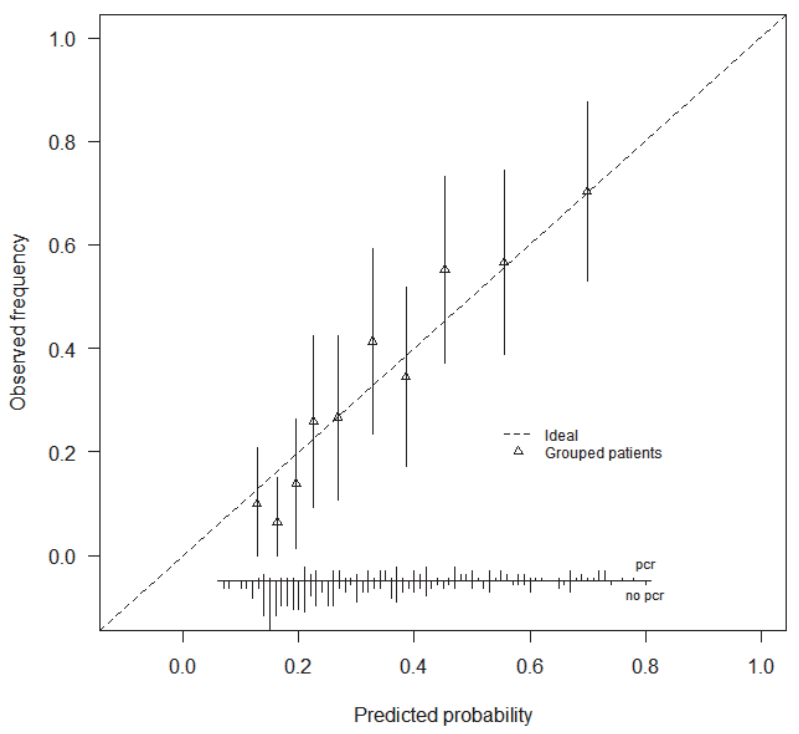

Figure 6.2 Calibration plot after internal validation by bootstrapping $(n=1000)$.

In this figure the calibration plot of the prediction model is presented. The diagonal would represent a perfect model where the predicted probability by the prediction model ( $\mathrm{x}$-axis) perfectly matches the actual probability as observed by pathologic examination ( $y$-axis). Patients were divided into deciles according to their predicted probability/nomogram score. The dots represent the calculated probability (nomogram score/predicted probability) compared to the observed probability.

\section{Optimal cut-off value for nomogram score/predicted probability}

Sensitivity and specificity for predicting axillary $\mathrm{PCR}$ at different cutoff values are summarized in Table 6.5. At a cutoff value of $\geq 0.50$, specificity is $88 \%$ and sensitivity is $43 \%$. Although higher cutoff values result in higher specificity, sensitivity rapidly falls to a point where the model only identifies a quarter of patients in which ALND may be omitted. 


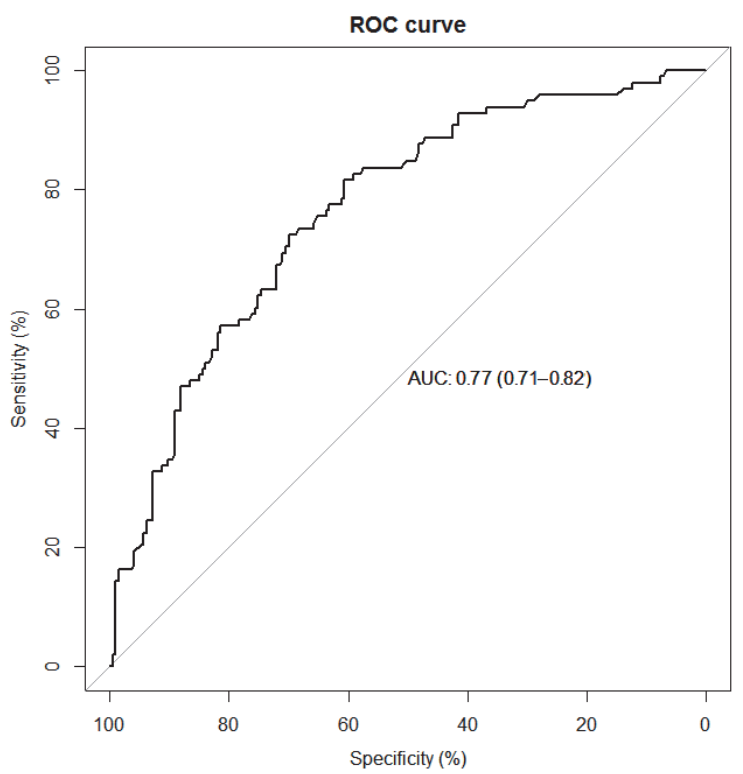

Figure 6.3 Receiver Operating Characteristic (ROC) plot after internal validation by bootstrapping $(n=1000)$.

This figure represents the Receiver Operating Characteristic curve of the prediction model, representing the discriminative value of the regression model to predict axillary nodal pathological complete response. The ROC-curve corresponds with an area under the curve of 0.77 (95\% Cl 0.71-0.82).

\section{Performance in individual patients}

Positive predictive values at different cutoff values (associated with different sensitivities and specificities) at the observed axillary pCR prevalence of $34 \%$ are listed in Table 6.5. At a cutoff value of $\geq 0.50$, PPV is $65 \%$. Higher cut-off values only increase PPV marginally (to a maximum of $69 \%$ ), in addition to rapidly decreasing sensitivity.

\section{Discussion}

This study aimed to develop a prediction model to identify clinically node positive breast cancer patients who achieve axillary PCR after neoadjuvant chemo(-immuno) therapy, in order to select patients in whom ALND may be omitted without need for an invasive procedure. Patient and tumor characteristics of 291 clinically node positive breast cancer patients who underwent neoadjuvant chemo(-immuno)therapy and subsequent ALND were analyzed retrospectively. In summary, the model is reasonably accurate in terms of discriminative ability as reflected in the AUC of $0.77(95 \% \mathrm{Cl}$ 
0.71-0.82). However, omitting axillary treatment based on the nomogram score solely is not justified. At a cut-off value of a nomogram score of $\geq 0.50$, this model has a specificity of $88 \%$ and a PPV of $65 \%$ (at axillary pCR prevalence of $34 \%$ ) for the occurrence of axillary PCR. The sensitivity at this cut-off value is $43 \%$, and thus it only identifies almost half of the patients with axillary PCR in whom ALND may be omitted. The PPV of $65 \%$ leaves a significant degree of uncertainty about the occurrence of axillary $\mathrm{pCR}$, raising the question whether it is safe to omit ALND in patients identified by this model. The $35 \%$ chance of leaving residual tumor in lymph nodes behind is probably too high to decide against ALND in every patient. However, in specific cases, such as patients in whom complications of axillary surgery are a direct threat to their profession or leisure activities, the probability of $\mathrm{pCR}$ as determined by the prediction model might aid the decision whether or not to perform an ALND.

The model offers several advantages compared to alternative methods for assessing axillary $\mathrm{pCR}$ after neoadjuvant chemo(-immuno)therapy. First, in contrast to SLNB, prediction of probability of axillary $\mathrm{PCR}$ based on a model is non-invasive and therefore lacks the associated morbidity. Second, because all parameters are included in standard initial breast cancer workup, the model can be readily completed for each breast cancer patient before surgery, avoiding the need for additional diagnostic procedures. The model's third advantage is possible cost reduction related to eliminating the need for additional diagnostic tests or surgical procedures and pathologic examination of the lymph nodes.

Development of prediction models has limitations. Most importantly, external validation in a new group of patients is needed to confirm the diagnostic performance of a model ${ }^{26}$. Prediction models are often less accurate in external validation studies. External validation of this model is not warranted, as the suboptimal diagnostic performance limits clinical applicability. External validation is not expected to improve this sufficiently for clinical use.

The data reflect actual breast cancer care provided in five different Dutch hospitals over the past years indications for neoadjuvant chemo(-immuno)therapy, but indications for neoadjuvant systemic therapy have broadened, potentially changing the population characteristics. If this involves, for instance, a lower proportion of HER2 positive tumors, this could decrease the prevalence of axillary PCR and therefore alter the diagnostic performance of a model.

Furthermore, we did not perform a central review of the pathology specimens. Although a central review is considered appropriate for research purposes, our approach is more consistent with the targeted setting for the model. Finally, the model could be improved by adding more parameters. Proliferation scores such as the modified Bloom \& Richardson grade ${ }^{27}$ or Ki-67 might be relevant in this respect. However, the Bloom \& Richardson grade as determined by a core biopsy is not consistently representative for the grade determined by the tumor specimen after primary surgery ${ }^{18}$.Scoring Ki-67 has not yet become part of the standard breast cancer 
workup and suffers from high inter- and intra-observer variability ${ }^{28}$. Response of the primary tumor to neoadjuvant therapy (ypT status) was considered as an additional factor in the prediction model. It was not incorporated in the model because this parameter has the disadvantage that it is not available before surgery of the breast. If axillary surgery is warranted because axillary PCR is not achieved, a second operation is required. Furthermore, although a ypTO can be considered as a predictor for axillary $\mathrm{pCR}$, it is known that a discordance exists between tumor response and nodal response to neoadjuvant chemo(-immuno)therapy ${ }^{3,4}$. An explanation may be found in differences in tumor biology of breast cancer cells in metastatic lymph nodes compared to breast cancer cells in the primary tumor ${ }^{29,30}$.

In accordance with other studies on predictors of axillary PCR in clinically node positive breast cancer patients we found a higher rate of axillary $P C R$ in triple negative or HER2 positive tumors, and a lower axillary $\mathrm{PCR}$ rate in lobular carcinoma ${ }^{4}$. Surprisingly, a taxane based neoadjuvant systemic therapy regimen was associated with slightly lower axillary PCR rates in our population. This is in contrast with studies on $\mathrm{PCR}$ rates of the breast tumor, which showed a beneficial effect of taxane based neoadjuvant systemic therapy regimens. This may be explained by either the small number of patients in our population receiving a non-taxane based neoadjuvant systemic therapy regimen $(n=63)$, or again by differences in tumor biology of the primary breast tumor and its nodal metastases.

In contrast to what one might expect, invasive or radiologic restaging methods do not consistently perform better than this prediction model. Several techniques have been suggested for restaging of initially positive axillary lymph nodes after neoadjuvant chemo(-immuno)therapy. SLNB correctly determines nodal status in $61-84 \%$ of cases, based on $80-94 \%$ identification and $10-20 \%$ false negative SLNBs ${ }^{1,8-11}$. Besides SLNB, the MARI-procedure (Marking the Axilla with Radioactive lodine seeds) is under investigation. This procedure involves placing an iodine seed prior to neoadjuvant treatment, marking the ultrasound positive axillary node, which will be removed after neoadjuvant treatment, as indicator of axillary response. The MARI thereby procedure overcomes the problem of identifying a (representative) lymph node. However, only limited data are available on the diagnostic accuracy of the MARI-procedure ${ }^{31}$. Furthermore, both the SLNB and MARI-procedure are invasive procedures with possible short and long-term morbidities. Physical examination and ultrasound have limited PPVs (corrected for the prevalence of axillary PCR in this study of $34 \%$ ) of $35 \%$ and $42 \%$, respectively. Further diagnostic values are summarized in Table $6.1^{1,2}$. As a non-invasive tool, PET-CT seems more promising. For everyday clinical use in the individual patient, only a decrease in maximum standardized uptake value $\left(S U V_{\max }\right)$ on PET-CT early in the course of neoadjuvant chemo(-immuno)therapy, compared to SUV $\mathrm{Sax}_{\max }$ on baseline PET-CT seems reliable. A relative decrease of $\geq 60 \%$ of $S U V_{\max }$ on PET-CT after 2-3 weeks of neoadjuvant chemo(-immuno)therapy shows a sensitivity of $48 \%$ and positive predictive value (PPV) of $86 \%$ for identifying axillary pCR in node positive patients after 
neoadjuvant chemo(-immuno)therapy (at prevalence of $40 \%)^{2}$. No studies have been performed yet on dedicated contrast enhanced axillary MRI for this purpose, though it might be useful; Lambregts et al. have shown the feasibility of nodal restaging with non-invasive gadofosveset enhanced MRI in rectal cancer patients after neoadjuvant chemoradiation therapy. Gadofosveset enhanced MRI evaluated by a senior radiologist showed a sensitivity of $81 \%$ and a specificity of $96 \%$ for detecting pCR of lymph nodes after neoadjuvant therapy in rectal cancer ${ }^{32}$. The diagnostic accuracy of gadofosveset enhanced MRI for restaging nodal status after neoadjuvant treatment in breast cancer is not known. However, gadofosveset enhanced MRI of the axillary lymph nodes seems promising for primary nodal staging purposes, with a sensitivity and specificity of $86 \%$ and $94 \%$, respectively ${ }^{33}$.

\section{Conclusion}

In conclusion, our prediction model outperforms clinical examination and axillary ultrasound for evaluating axillary lymph node status after neoadjuvant chemo(immuno)therapy. It shows reasonable accuracy with an area under the ROC curve of 0.77 . However, omitting axillary treatment based on the nomogram score solely is not justified. Further research is needed to identify patients with axillary pCR using noninvasive techniques, in order to select patients in whom ALND may be omitted.

\section{Clinical practice points}

- Between $20-42 \%$ of clinically node positive breast cancer patients who undergo neoadjuvant chemotherapy or immunotherapy achieve pathologic complete response of the axillary lymph nodes. Hypothetically, axillary lymph node dissection may be safely omitted in these patients.

- There is no reliable method is available to confirm axillary pCR preoperatively. Noninvasive techniques that have been studied for this purpose include physical examination and ultrasound, which lack both sensitivity and specificity. PET-CT shows sufficient specificity but lacks sensitivity.

- A prediction model was developed for identifying patients achieving axillary pCR noninvasively. This prediction model is based on patient and tumor characteristics of 291 clinical node positive breast cancer patients treated with neoadjuvant chemo(immuno)therapy. The model is reasonably accurate as reflected in the AUC of 0.77 (95\% $\mathrm{Cl} 0.71-0.82$ ). At a cut-off value of a nomogram score of $\geq 0.50$, this model has a specificity of $88 \%$ and a PPV of $65 \%$ (prevalence of axillary pCR of $34 \%$ ) for identifying axillary pCR. The sensitivity at this cut-off value is $43 \%$, and thus it only identifies almost half of the patients in whom ALND may be omitted. 
- The PPV of $65 \%$ limits the use of this model in clinical practice; the $35 \%$ chance of leaving metastatic lymph nodes behind is probably too high to omit ALND in most patients. Further research is needed to identify patients with axillary PCR using noninvasive techniques, in order to select patients in whom ALND may be omitted. 


\section{References}

1. Alvarado R, Yi M, Le-Petross H, Gilcrease M, Mittendorf EA, Bedrosian I, Hwang RF, Caudle AS, Babiera GV, Akins JS, Kuerer HM, Hunt KK. The role for sentinel lymph node dissection after neoadjuvant chemotherapy in patients who present with node-positive breast cancer. Ann Surg Oncol. 2012;19:3177-84.

2. Koolen BB, Valdés Olmos RA, Wesseling J, Vogel WV, Vincent AD, Gilhuijs KG, Rodenhuis S, Rutgers EJ, Vrancken Peeters MJ. Early assessment of axillary response with ${ }^{18} \mathrm{~F}-\mathrm{FDG}$ PET/CT during neoadjuvant chemotherapy in stage II-III breast cancer: implications for surgical management of the axilla. Ann Surg Oncol. 2013;20:2227-35.

3. Rouzier R, Extra JM, Klijanienko J, Falcou MC, Asselain B, Vincent-Salomon A, Vielh P, Bourstyn E. Incidence and prognostic significance of complete axillary downstaging after primary chemotherapy in breast cancer patients with T1 to T3 tumors and cytologically proven axillary metastatic lymph nodes. J Clin Oncol. 2002;20:1304-10.

4. Straver ME, Rutgers EJ, Russell NS, Oldenburg HS, Rodenhuis S, Wesseling J, Vincent A, Peeters MT. Towards rational axillary treatment in relation to neoadjuvant therapy in breast cancer. Eur J Cancer. 2009;45:2284-92.

5. von Minckwitz G, Untch M, Blohmer JU, Costa SD, Eidtmann H, Fasching PA, Gerber B, Eiermann W, Hilfrich J, Huober J, Jackisch C, Kaufmann M, Konecny GE, Denkert C, Nekljudova V, Mehta K, Loibl S. Definition and impact of pathologic complete response on prognosis after neoadjuvant chemotherapy in various intrinsic breast cancer subtypes. J Clin Oncol. 2012;30:1796-804.

6. Ashikaga T, Krag DN, Land SR, Julian TB, Anderson SJ, Brown AM, Skelly JM, Harlow SP, Weaver DL, Mamounas EP, Costantino JP, Wolmark N; National Surgical Adjuvant Breast, Bowel Project. Morbidity results from the NSABP B-32 trial comparing sentinel lymph node dissection versus axillary dissection. J Surg Oncol. 2010;102:111-8.

7. Liu CQ, Guo Y, Shi JY, Sheng Y. Late morbidity associated with a tumour-negative sentinel lymph node biopsy in primary breast cancer patients: a systematic review. Eur J Cancer. 2009;45:1560-8.

8. Gimbergues P, Abrial C, Durando X, Le Bouedec G, Cachin F, Penault-Llorca F, Mouret-Reynier MA, Kwiatkowski F, Maublant J, Tchirkov A, Dauplat J. Sentinel lymph node biopsy after neoadjuvant chemotherapy is accurate in breast cancer patients with a clinically negative axillary nodal status at presentation. Ann Surg Oncol. 2008;15:1316-21.

9. Pecha V, Kolarik D, Kozevnikova R, Hovorkova K, Hrabetova P, Halaska M, Sottner O, Trnkova M, Petruzelka L, Kolarova H. Sentinel lymph node biopsy in breast cancer patients treated with neoadjuvant chemotherapy. Cancer. 2011;117:4606-16.

10. Boughey JC. The role of sentinel lymph node surgery in patients presenting with node positive breast cancer (T0-T4, N1-2) who receive neoadjuvant chemotherapy - results from the ACOSOG Z1071 trial. San Antonio Breast Cancer Symposium; 2012, San Antonio.

11. Kuehn T, Bauerfeind I, Fehm T, Fleige B, Hausschild M, Helms G, Lebeau A, Liedtke C, von Minckwitz G, Nekljudova V, Schmatloch S, Schrenk P, Staebler A, Untch M. Sentinel-lymph-node biopsy in patients with breast cancer before and after neoadjuvant chemotherapy (SENTINA): a prospective, multicentre cohort study. Lancet Oncol. 2013;14:609-18.

12. Schijven MP, Vingerhoets AJ, Rutten HJ, Nieuwenhuijzen GA, Roumen RM, van Bussel ME, Voogd AC. Comparison of morbidity between axillary lymph node dissection and sentinel node biopsy. Eur J Surg Oncol. 2003;29:341-50.

13. NABON. Guideline Breast Carcinoma 2004 The Netherlands. 2004.

14. NABON. Guideline Breast Carcinoma 2008 The Netherlands. 2008.

15. NABON. Guideline Breast Carcinoma 2012 The Netherlands. 2012.

16. Palmieri C, Jones A. The 2011 EBCTCG polychemotherapy overview. Lancet. 2012;379:390-2.

17. Harris GC, Denley HE, Pinder SE, et al. Correlation of histologic prognostic factors in core biopsies and therapeutic excisions of invasive breast carcinoma. Am J Surg Pathol. Jan 2003;27(1):11-15.

18. O'Shea AM, Rakha EA, Hodi Z, Ellis IO, Lee AH. Histological grade of invasive carcinoma of the breast assessed on needle core biopsy - modifications to mitotic count assessment to improve agreement with surgical specimens. Histopathology. 2011;59:543-8. 
19. Mazouni C, Peintinger F, Wan-Kau S, Andre F, Gonzalez-Angulo AM, Symmans WF, Meric-Bernstam F, Valero V, Hortobagyi GN, Pusztai L. Residual ductal carcinoma in situ in patients with complete eradication of invasive breast cancer after neoadjuvant chemotherapy does not adversely affect patient outcome. J Clin Oncol. 2007;25:2650-5.

20. Fumagalli D, Bedard PL, Nahleh Z, Michiels S, Sotiriou C, Loi S, Sparano JA, Ellis M, Hylton N, Zujewski JA, Hudis C, Esserman L, Piccart M; BIG-NABCG collaboration. A common language in neoadjuvant breast cancer clinical trials: proposals for standard definitions and endpoints. Lancet Oncol. 2012;13:e240-8.

21. Sakakibara M, Nagashima T, Kadowaki M, Onai Y, Fujimori T, Yokomizo J, Suzuki H, Fushimi K, Nakatani $Y$, Miyazaki M. Clinical significance of axillary microresiduals after neoadjuvant chemotherapy in breast cancer patients with cytologically proven metastases. Ann Surg Oncol. 2009;16:2470-8.

22. Efron B. Bootstrap Methods: Another Look at the Jackknife. Ann. Statist. 1979 1979;7:1-26.

23. Steyerberg EW, Harrell FE, Jr., Borsboom GJ, Eijkemans MJ, Vergouwe Y, Habbema JD. Internal validation of predictive models: efficiency of some procedures for logistic regression analysis. J Clin Epidemiol. 2001;54:774-81.

24. Harrell FE. Regression modeling strategies : with applications to linear models, logistic regression, and survival analysis. New York: Springer; 2001.

25. Steyerberg EW, Vickers AJ, Cook NR, Gerds T, Gonen M, Obuchowski N, Pencina MJ, Kattan MW. Assessing the performance of prediction models: a framework for traditional and novel measures. Epidemiology. 2010;21:128-38.

26. Babyak MA. What you see may not be what you get: a brief, nontechnical introduction to overfitting in regression-type models. Psychosom Med. 2004;66:411-21.

27. Elston CW, Ellis IO. Pathological prognostic factors in breast cancer. I. The value of histological grade in breast cancer: experience from a large study with long-term follow-up. Histopathology. 2002;41: 154-61.

28. Varga Z, Diebold J, Dommann-Scherrer C, Frick H, Kaup D, Noske A, Obermann E, Ohlschlegel C, Padberg B, Rakozy C, Sancho Oliver S, Schobinger-Clement S, Schreiber-Facklam H, Singer G, Tapia C, Wagner U, Mastropasqua MG, Viale G, Lehr HA. How reliable is Ki-67 immunohistochemistry in grade 2 breast carcinomas? A QA study of the Swiss Working Group of Breast- and Gynecopathologists. PLoS One. 2012;7:e37379.

29. Dikicioglu E, Barutca S, Meydan N, Meteoglu I. Biological characteristics of breast cancer at the primary tumour and the involved lymph nodes. Int J Clin Pract. 2005;59:1039-44.

30. Sjostrom-Mattson J, Von Boguslawski K, Bengtsson NO, Mjaaland I, Salmenkivi K, Blomqvist C. The expression of $\mathrm{p} 53, \mathrm{bcl}-2$, bax, fas and fas $\mathrm{L}$ in the primary tumour and lymph node metastases of breast cancer. Acta oncologica. 2009;48:1137-43.

31. Straver ME, Loo CE, Alderliesten T, Rutgers EJ, Vrancken Peeters MT. Marking the axilla with radioactive iodine seeds (MARI procedure) may reduce the need for axillary dissection after neoadjuvant chemotherapy for breast cancer. Br J Surg. 2010;97:1226-31.

32. Lambregts DM, Beets GL, Maas M, Kessels AG, Bakers FC, Cappendijk VC, Engelen SM, Lahaye MJ, de Bruïne AP, Lammering G, Leiner T, Verwoerd JL, Wildberger JE, Beets-Tan RG.. Accuracy of gadofosveset-enhanced MRI for nodal staging and restaging in rectal cancer. Ann Surg. 2011;253: 539-45.

33. Schipper RJ, Smidt ML, van Roozendaal LM, Castro CJ, de Vries B, Heuts EM, Keymeulen KB, Wildberger $\mathrm{JE}$, Lobbes MB, Beets-Tan RG. Noninvasive nodal staging in patients with breast cancer using gadofosveset-enhanced magnetic resonance imaging: a feasibility study. Invest Radiol. 2013;48:134-9. 


\section{Chapter}

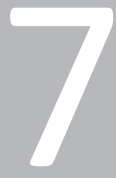

The diagnostic performance of gadofosvesetenhanced axillary MRI for nodal (re-)staging in breast cancer patients : can the initial promising results be reproduced?

RJ. Schipper

M.L. Smidt

L.M. van Roozendaal

P.J. Nelemans

M. Paiman

B.T. Brans

M. Moossdorff

B. de Vries

K.B.M.I. Keymeulen

J.E. Wildberger

M.B.I. Lobbes

R.G.H. Beets-Tan

Submitted 


\section{Abstract}

\section{Objective}

To evaluate the diagnostic performance of gadofosveset-enhanced MRI for nodal (re-)staging in newly diagnosed patients with breast cancer.

\section{Materials and methods}

Seventy-three patients underwent axillary MRI, consisting of standard and gadofosvesetenhanced MRI (GDF-MRI). Two radiologists independently scored each lymph node on a confidence level scale, first on standard MRI, subsequently adjusting their score based on the GDF-MRI. Diagnostic performance parameters were calculated based on a node-by-node and patient-by-patient validation with histopathology as gold standard. To investigate the presence of a learning curve for reading GDF-MRI, the diagnostic performance on the first 50 included patients was compared to the last 23.

\section{Results}

Overall, node-by-node validation, for reader 1 , standard and GDF-MRI showed similar diagnostic performance with an AUC of 0.78 and $0.80(P=0.442)$, respectively, while for reader 2 it was 0.79 and $0.71(P=0.007)$. For patient-by-patient validation, the AUC of standard and GDF-MRI were 0.89 and $0.82(P=0.295)$ and 0.81 and $0.77(P=0.506)$ for reader 1 and 2 , respectively.

The AUC for reader 1 of the first 50 cases was 0.75 , improving to $0.94(P=0.09)$ for the remaining 23 cases. Sensitivity, specificity, PPV and NPV improved from $67 \%, 82 \%, 53 \%$, and $89 \%$ to $83 \%$, $94 \%, 83 \%$ and $94 \%$, respectively. The AUC of reader 2 improved from 0.75 to 0.80 ( $P=0.783$ ).

\section{Conclusion}

The current study confirmed previous study that GDF-MRI has potential as a noninvasive method for nodal (re-)staging in breast cancer. However a learning curve exists for especially breast radiologists who are not familiar with GDF-MRI. 


\section{Introduction}

Breast cancer is one of the most frequently diagnosed cancers among women, with a worldwide incidence rate of 1.38 million $^{1}$. Survival rates have increased in the last decades due to improved diagnostic techniques, more adequate surgery and more extensive systemic and radiotherapy treatment regimens. Five year survival rates are up to $98 \%$ for early stage, lymph node negative breast cancer ${ }^{2}$. Consequently, there is more attention for maintaining quality of life by limiting overtreatment and its associated lifetime morbidity.

For a long time, axillary lymph node dissection (ALND) was routinely performed to assess nodal status in patients with breast cancer. This procedure is associated with significant short- and long-term morbidity. Seroma, lymphedema, nerve injury and reduced shoulder function are reported in up to $49 \%$ of the patients after 3 years of follow-up ${ }^{3-5}$. About 15 years ago, the sentinel lymph node biopsy (SLNB) became the standard procedure, which is a less invasive technique to evaluate nodal status in patients with clinically node negative breast cancer, followed by a completion axillary lymph node dissection in case of a positive sentinel lymph node $(s)^{6}$. The SLNB, however, is still an invasive procedure with short-term surgical side effects in $25 \%$ of patients and long-term morbidity (for example lymphedema) in up to $8 \%$ of the patients after only three years ${ }^{4,7,8}$.

Since $74 \%$ of the sentinel lymph nodes show no metastasis, a noninvasive imaging technique able to identify these node negative patients would be a step forward in personalized treatment by reducing overtreatment ${ }^{9}$. It would result in a significant reduction of morbidity, increasing the patient's quality of life.

An accurate noninvasive nodal staging tool could also guide treatment in patients with clinically node positive breast cancer. In case of neoadjuvant systemic therapy, pathological complete response of axillary lymph node metastases is achieved in $20-42 \%{ }^{10-13}$. Identifying these patients by a noninvasive imaging technique, and thereby avoiding an ALND, would further reduce breast cancer treatment related morbidity.

A feasibility study on the use of magnetic resonance imaging (MRI) with a bloodpool contrast agent, gadofosveset, for axillary nodal staging in ten patients with breast cancer showed a sensitivity, specificity, positive predictive value (PPV) and negative predictive value (NPV) $86 \%, 94 \%, 75 \%$ and $97 \%$, respectively, based on a node-by-node analysis of MRI findings to histopathology. It was concluded that gadofosvesetenhanced $\mathrm{MRI}$ is a promising tool to accurately detect patients with node negative breast cancer ${ }^{14}$.

The aim of this study was to prospectively assess the diagnostic performance of gadofosveset-enhanced MRI for nodal (re-)staging in a larger population of patients with newly diagnosed breast cancer by a per node and per patient analysis using histopathology as gold standard. 


\section{Materials and methods}

\section{Setting and patients}

The local medical ethics committee approved this prospective single center study. Consecutive patients with breast cancer between August 2012 and May 2014 were included after written informed consent was obtained. Eligible for inclusion were patients with biopsy proven in situ or invasive breast cancer to be operated by SLNB or ALND. Exclusion criteria were pregnancy, prior ipsilateral axillary surgery, a glomerular filtration rate $<45 \mathrm{ml} / \mathrm{min} / 1.73 \mathrm{~m}^{2}$, and contra-indications for either MRI or the gadofosveset contrast agent. The gadofosveset-enhanced axillary MRI was performed shortly before SLNB or ALND in standard setting. In case of neoadjuvant systemic therapy, axillary MRI was performed between the last course of systemic therapy and SLNB and/or ALND.

\section{Imaging}

Axillary imaging was performed on a 3.0 Tesla MRI scanner (Achieva 3.0TX, Philips Healthcare, Best, The Netherlands) using a 32-channel cardiac sensitivity encoding coil with the patient in a supine position and ipsilateral arm elevated. The imaging protocol consisted of the following parameters: First, a three-dimensional (3D) standard T2 weighted (T2W) turbo spin echo sequence without fat suppression was performed in 3 minutes 26 seconds (TR/TE 2000/153 msec; 90 degree flip angle; turbo spin echo factor 50; NSA 1; slice thickness 2.50-mm; reconstruction section thickness $1.25-\mathrm{mm}$; field of view $220 \times 220 \mathrm{~mm}^{2}$; matrix $176 \times 176$; resulting in an in-plane resolution of $1.25 \times 1.25 \mathrm{~mm}^{2}$ ).

Second, gadofosveset (Ablavar ${ }^{\complement}$, Lantheus Medical Imaging, North Billerica, United States of America) contrast with a dose of $0.12 \mathrm{ml} / \mathrm{kg}$ bodyweight was intravenously administered, followed after 12 minutes by a 3D T1 weighted (T1W) gradient echo sequence in 5 minutes 7 seconds (TR/TE 6.3/4.6 milliseconds; 15 degree flip angle; NSA 2; slice thickness $1.25 \mathrm{~mm}$; field-of-view $220 \times 220 \mathrm{~mm}^{2}$; matrix size $176 \times 176$; resulting in an in-plane resolution of $1.25 \times 1.25 \mathrm{~mm}^{2}$ ). The time interval of 12 minutes after contrast administration was chosen based on previous publication ${ }^{14}$.

\section{Image evaluation}

Two radiologists independently analyzed standard T2W MRI and standard T2W MRI + gadofosveset-enhanced MRI (GDF-MRI), blinded for histopathological findings and each other's reading results. Similar to clinical practice, the radiologists were aware of the clinical tumor size, assessed on ultrasound/mammography and/or breast MRI. However, they were blinded for the results of physical examination of the axilla and results of axillary ultrasound. 
The first radiologist (RBT) has 15 years of experience in pelvic MRI including six years in gadofosveset-enhanced MRI for lymph node assessment in rectal cancer specifically [reader 1]. The second radiologist (ML) has five years of experience in breast MRI and in axillary lymph node assessment using ultrasound and two years in axillary lymph node assessment on breast MRI [reader 2].

On standard axillary MRI, each node was scored using a confidence level scale of 0 (definitely benign) to 4 (definitely malignant), using the criteria as described by Baltzer et al. $^{15}$. Characteristics indicating malignancy were irregular margins, inhomogeneous cortex, perifocal edema and absent fatty hilum. Next, GDF-MRI images were presented to the radiologist for analysis, and the radiologists were allowed to adjust their initial score. The GDF-MRI images were scored based on the criteria as described by Lambregts et al. ${ }^{16}$; in short, a node was considered benign when it showed contrast hyperintensity after intravenous injection of gadofosveset contrast. Malignant characteristics were absence of contrast hyperintensity and the absence of an intact nodal border.

\section{Surgery}

The sentinel lymph node was depicted on lymphoscintigraphy by using technetium$99 \mathrm{~m}$ nanocolloid as a radioactive tracer. During surgery, the sentinel lymph node was identified with triple technique consisting of preoperative lymphoscintigraphy, 1-2 ml of blue dye (Bleu Patente V; Guerbet, Aulnay-sous-Bois, France) and detection of the radioactivity by gamma probe.

If an (completion) ALND of level I and II was performed, the following anatomical landmarks were marked: caudal-medial edge, caudal-lateral edge and cranial-lateral edges of the specimen. A marker was placed at the branching of the lateral thoracic vein from the axillary vein. Finally, the medial apex of axilla was marked.

\section{Histopathological evaluation}

For histological evaluation of sentinel lymph nodes, the nodes were sliced with a maximum slice thickness of $3 \mathrm{~mm}$ and embedded in paraffin. Each part was step sectioned at 500-micrometer intervals at three levels and stained with haematoxylin and eosin (H\&E). Cytokeratin immunohistochemical staining, using a mouse anti-human MNF116 antibody (Dako, Glostrup, Denmark), was performed if conventional H\&E slices were negative.

For histological evaluation in case of an (completion) ALND, the ALND specimen was received immediately from the operating room. Before formalin fixation, the specimen was fixed in a box representing the normal anatomical situation in the patient. All palpable lymph nodes were embedded in paraffin after 24 hours of fixation. Lymph nodes bigger than $5 \mathrm{~mm}$ were sliced with a maximum slice thickness of $3 \mathrm{~mm}$, and stained with $H \& E^{17}$. 
Each node was recorded as either benign (pNO) or as isolated tumor cells (ITC) (pNO(i+)) $(\leq 0.2 \mathrm{~mm})$, micrometastasis $(\mathrm{pN} 1 \mathrm{mi})(0.2 \leq 2.0 \mathrm{~mm})$ or macrometastasis (pN1-3) $(>2.0 \mathrm{~mm})$. Isolated tumor cells (ITC) and micrometastases detected by histopathological analysis were considered negative.

\section{Node-by-node evaluation}

A SPECT-CT scan was used for matching of the sentinel lymph nodes removed during surgery and imaged by axillary MRI. To achieve a reliable match between the ALND specimen and axillary MRI, every node harvested by the pathologist was drawn on an anatomical map. In this way, the location of a particular lymph node in the ALND specimen could be matched with the location on axillary MRI, thereby allowing nodeby-node analysis. This matching procedure was described in detail in two earlier publications $^{14}$.

\section{Statistical analyses}

Statistical analyses were performed using Statistical Package for the Social Sciences (version 22.0; IBM, Armonk, New York, United States of America) and R Project (version 3.0.1; R Foundation for Statistical Computing, Vienna, Austria).

In patients treated with SLNB, only the lymph nodes on axillary MRI identified as sentinel lymph nodes were taken into account for the patient-by-patient analysis. In patients treated with ALND, all lymph nodes depicted on axillary MRI were included into the patient-by-patient analysis.

Diagnostic performance parameters, including sensitivity, specificity, positive predictive value (PPV), negative predictive value (NPV) and area under the receiver operating characteristic (ROC) curve (AUC), were analyzed for standard MRI and standard MRI + GDF-MRI. At the start of the study, the cut-off on the confidence level scale was defined between 2 (possibly benign) and 3 (probably malignant), with lymph nodes scored 2 or lower categorized as benign and those scored 3 or higher as malignant.

Since lymph nodes are clustered per patient when reading axillary MRI, lymph node observations within one patient cannot be regarded as independent observations. To adjust for this within-patient correlation, bootstrapping was performed ( $n=1000$ repetitions) for calculation of the $95 \%$ confidence intervals for the diagnostic performance parameters) $)^{18}$.

After the first 50 patients, both readers were informed about their diagnostic performance and feedback was provided on false positive and false negative cases. To investigate the presence of a learning curve for reading the GDF-MRI images, all the diagnostic performance parameters of the first two-third (i.e. before providing feedback on performance) of included patients were compared to the last one-third of included patients. 
Differences in diagnostic performance parameters between standard MRI and standard MRI + GDF-MRI and between the first two-third and last one-third of included patients were tested for statistical significance. For the patient-by-patient validation a nonparametric test for comparing correlated receiver operating characteristic curves was used ${ }^{19}$.

$P$-values $<0.05$ were considered to indicate statistical significance.

\section{Results}

\section{Patients}

During the study period, 76 patients were included and underwent gadofosvesetenhanced axillary MRI. No serious adverse events were observed regarding contrast administration. Three patients were excluded; one since no axillary lymph nodes were surgically removed, one because the histopathology of the axillary lymph nodes showed chronic lymphocytic leukemia, and one for the presence of silicon adenitis in the axillary lymph nodes. Consequently, the results of 73 patients were included in the final analysis. Patient and tumor characteristics are summarized in Table 7.1. Sixty-five patients underwent SLNB or ALND first; the remaining eight were treated with neoadjuvant systemic therapy. Median time interval between axillary MRI and surgery was five days (range 0-30 days).

\section{Histopathology}

A total of 328 lymph nodes were removed during SLNB (117 nodes) and ALND (211 nodes), of which 237 could be matched to lymph nodes identified on the standard MRI. Histopathological examination of the 237 lymph nodes resulted in 73 macrometastases (31\%), and 164 negative lymph nodes (including 5 ITC and 16 micrometastases). On patient level, at least one axillary macrometastasis was detected in 18 of the 73 patients (25\%). Findings on axillary MRI compared to histopathology are presented in Table 7.2.

\section{Diagnostic performance for the detection of nodal metastases: node-by- node analysis of the entire study population}

For reader 1, standard MRI showed an AUC of 0.78 and standard MRI + GDF-MRI an AUC of $0.80(P=0.442)$, For reader 2 the AUC of standard MRI was 0.79 , whereas the AUC of standard MRI + GDF-MRI was $0.71(P=0.007)$. The diagnostics parameters with 95\%-confidence intervals (95\% Cls) are presented in Table 7.3. 
Table 7.1 Patient demographics and tumor characteristics.

\begin{tabular}{|c|c|}
\hline Characteristic & Value \\
\hline Number of Patients & 73 \\
\hline \multicolumn{2}{|l|}{ Age, years } \\
\hline Mean (SD) & $59(12)$ \\
\hline Range & $22-80$ \\
\hline \multicolumn{2}{|l|}{ Clinical tumor size in situ carcinoma, $\mathrm{mm}(\mathrm{n}=4)$} \\
\hline Mean $(S D)$ & $40(30)$ \\
\hline Range & $24-85$ \\
\hline \multicolumn{2}{|l|}{ Clinical tumor size invasive carcinoma, $\mathrm{mm}(\mathrm{n}=69)$} \\
\hline Mean (SD) & $21(11)$ \\
\hline Range & $6-64$ \\
\hline \multicolumn{2}{|l|}{ cT-stadium, n (\%) } \\
\hline Tis & $4(5)$ \\
\hline $\mathrm{T} 1$ & $38(52)$ \\
\hline $\mathrm{T} 2$ & $29(40)$ \\
\hline T3 & $2(3)$ \\
\hline \multicolumn{2}{|l|}{ pN-stadium, n (\%) } \\
\hline pNO & $43(59)$ \\
\hline $\mathrm{pNO}(\mathrm{i}+)$ & $3(4)$ \\
\hline pN1mi & $5(7)$ \\
\hline pN1 & $9(13)$ \\
\hline pN2 & $4(6)$ \\
\hline pN3 & $2(3)$ \\
\hline ypNO & $3(4)$ \\
\hline ypN1mi & $1(1)$ \\
\hline ypN1 & $1(1)$ \\
\hline ypN2 & $1(1)$ \\
\hline ypN3 & $1(1)$ \\
\hline \multicolumn{2}{|l|}{ Tumor type, n (\%) } \\
\hline Ductal carcinoma in situ & $4(6)$ \\
\hline Ductal & $54(74)$ \\
\hline Lobular & $11(15)$ \\
\hline Other & $4(6)$ \\
\hline \multicolumn{2}{|l|}{ Uni-/multifocal tumors, n (\%) } \\
\hline Unifocal & $59(81)$ \\
\hline Multifocal and/or multicentric & $14(19)$ \\
\hline \multicolumn{2}{|l|}{ Hormone receptor and HER2 status, n (\%) } \\
\hline Triple negative & $9(12)$ \\
\hline ER/PR + HER2 - & $53(73)$ \\
\hline ER/PR - HER2 + & $3(4)$ \\
\hline $\mathrm{ER} / \mathrm{PR}+\mathrm{HER} 2+$ & $4(6)$ \\
\hline Not determined (in case of in situ carcinoma) & $4(6)$ \\
\hline
\end{tabular}

$\mathrm{N}$ : number of cases; SD: standard deviation; ER: estrogen receptor; PR: progesterone receptor; HER2: human epidermal growth factor receptor 2.

\section{Diagnostic performance: patient-by-patient analysis of the entire study population}

For reader 1 the AUC of standard MRI and standard MRI + GDF-MRI were 0.89 and 0.82 $(P=0.295)$ resp. and 0.81 and $0.77(P=0.506)$ for reader 2 . The corresponding diagnostic parameters and $95 \% \mathrm{Cls}$ are presented in Table 7.3. 
Table 7.2 Lymph node scoring on axillary MRI and findings by histopathology.

\begin{tabular}{|c|c|c|c|c|c|c|}
\hline & & & \multicolumn{4}{|c|}{ Histopathology } \\
\hline & & & Benign & Isolated tumor cells & Micrometastasis & Macrometastasis \\
\hline \multirow[t]{4}{*}{ R1 } & Standard MRI & - & 125 & 5 & 12 & 29 \\
\hline & & + & 18 & 0 & 4 & 44 \\
\hline & Standard + GDF-MRI & - & 124 & 5 & 11 & 23 \\
\hline & & + & 19 & 0 & 5 & 50 \\
\hline \multirow[t]{4}{*}{$\mathrm{R} 2$} & Standard MRI & - & 135 & 4 & 16 & 34 \\
\hline & & + & 8 & 1 & 0 & 39 \\
\hline & Standard + GDF-MRI & - & 130 & 5 & 13 & 38 \\
\hline & & + & 13 & 0 & 3 & 35 \\
\hline
\end{tabular}

R1: radiologist 1; R2: radiologist 2; Standard MRI = T2-weighted MRI; GDF-MRI: gadofosveset-enhanced T1-weighted MRI. Absolute numbers are presented.

Table 7.3 Diagnostic performance for prediction of axillary nodal status.

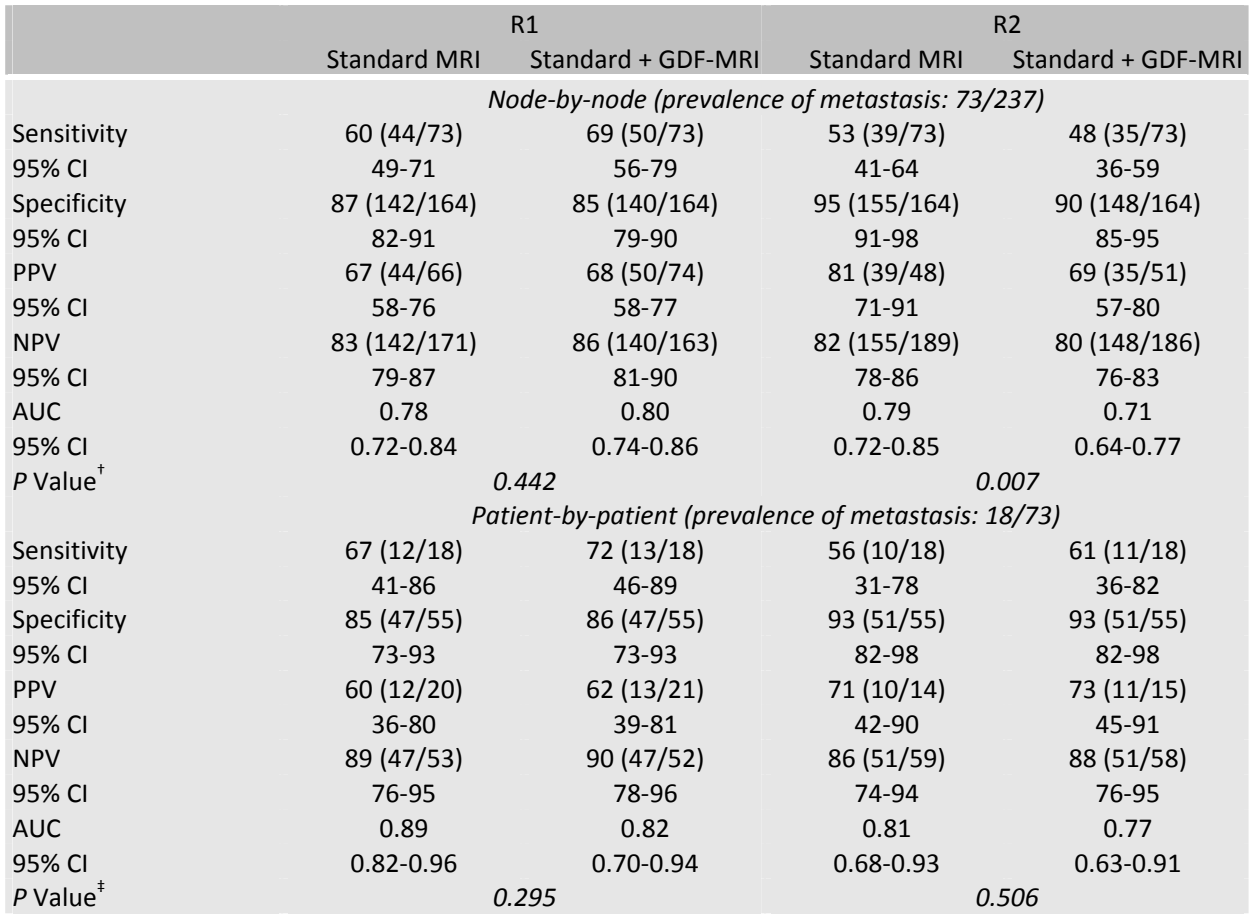

$\mathrm{R} 1$, radiologist 1; R2, radiologist 2; Standard MRI = T2-weighted MRI; GDF-MRI, gadofosveset-enhanced T1weighted $\mathrm{MRI}$; 95\% Cl, 95\% confidence interval; PPV, positive predictive value; NPV, negative predictive value; AUC, area under the curve; numbers are percentages, absolute numbers are given in parentheses.

${ }^{+} P$ Value for difference in AUC between standard and GDF-MRI, based on bootstrap test $(n=1000) ;{ }^{\ddagger} P$ Value for difference in AUC between standard and GDF-MRI, based on DeLong's test.

\section{After breaking down the study group in two cohorts $n=1-50$ and $n=50-73$}

For reader 1, the AUC of standard MRI + GDF-MRI of the first 50 cases was 0.75 , while the AUC of the remaining 23 cases was $0.94(P=0.09)$. The AUC of reader 2 improved 
from 0.75 to $0.80(P=0.783)$. Corresponding ROC curves and $95 \% \mathrm{Cls}$ of the AUC are presented in Figure 7.1, and all corresponding diagnostic parameters with $95 \% \mathrm{Cls}$ are presented in Table 7.4 .

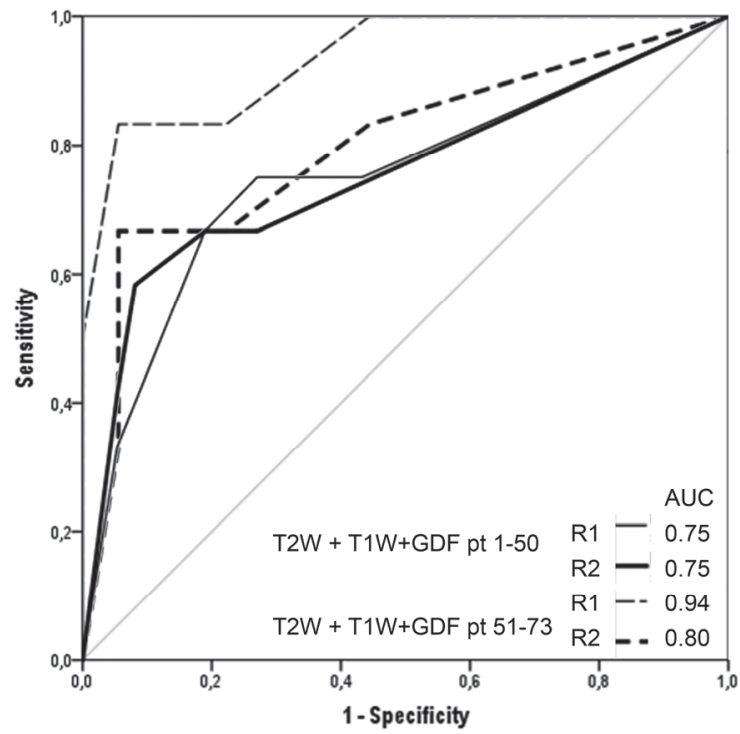

Figure 7.1 Receiver operating characteristics curves and areas under the curve (AUC) based on patient-bypatient analysis for detection of metastatic lymph nodes in the first 50 cases compared to the last 23 cases, using the combination of standard T2-weighted and gadofosveset-enhanced T1weighted (Standard + GDF-MRI) MRI for reader 1 and 2.

Table 7.4 Diagnostic performance for prediction of axillary nodal status patient-by-patient.

\begin{tabular}{|c|c|c|c|c|}
\hline & \multicolumn{2}{|c|}{ R1 Standard + GDF-MRI } & \multicolumn{2}{|c|}{ R2 Standard + GDF-MRI } \\
\hline & Patients 1-50 & Patients $51-73$ & Patients 1-50 & Patients $51-73$ \\
\hline Sensitivity & $67(8 / 12)$ & $83(5 / 6)$ & $58(7 / 12)$ & $67(4 / 6)$ \\
\hline $95 \% \mathrm{Cl}$ & $35-89$ & $36-99$ & 29-84 & $24-94$ \\
\hline Specificity & $82(31 / 38)$ & $94(16 / 17)$ & $92(35 / 38)$ & $94(16 / 17)$ \\
\hline $95 \% \mathrm{Cl}$ & 65-92 & $69-100$ & $78-98$ & $69-100$ \\
\hline PPV & $53(8 / 15)$ & $83(5 / 6)$ & $70(7 / 10)$ & $80(4 / 5)$ \\
\hline $95 \% \mathrm{Cl}$ & 27-78 & $36-99$ & $35-92$ & $30-99$ \\
\hline NPV & $89(31 / 35)$ & $94(16 / 17)$ & $88(35 / 40)$ & $89(16 / 18)$ \\
\hline $95 \% \mathrm{Cl}$ & $72-96$ & $69-100$ & $72-95$ & $64-98$ \\
\hline AUC & 0.75 & 0.94 & 0.75 & 0.80 \\
\hline $95 \% \mathrm{Cl}$ & $0.58-0.93$ & $0.82-1.00$ & $0.58-0.93$ & $0.56-1.00$ \\
\hline$P$ Value $^{\ddagger}$ & \multicolumn{2}{|c|}{0.090} & \multicolumn{2}{|c|}{0.783} \\
\hline
\end{tabular}

R1: radiologist 1; Standard MR = T2-weighted MRI; GDF-MRI: gadofosveset-enhanced T1-weighted MRI; R2: radiologist 2; 95\% $\mathrm{Cl}$ : $95 \%$ confidence interval; PPV: positive predictive value; NPV: negative predictive value; AUC: area under the curve; numbers are percentages, absolute numbers are given in parentheses. $\ddagger P$ Value for difference in AUC between patient group 1 (patients 1-50) and group 2 (patients 51-73), based on DeLong's test. 


\section{Discussion}

The aim of this prospective study was to evaluate the diagnostic performance of gadofosveset-enhanced MRI for nodal (re-)staging in patients with breast cancer and to assess whether the previously reported promising initial results can be reproduced in a second cohort of patients with newly diagnosed breast cancer. Both readers, regardless of their levels and background of expertise, showed comparable diagnostic performance of standard MRI (per patient AUC of 0.89 and 0.81 , respectively). The addition of GDF-MRI images did not improve their performances.

To understand whether a learning curve exists both readers had feedback on their errors after the first 50 set of patients. The reader who had the longest experience in nodal staging with GDF MRI (reader 1) significantly improved in performance after addition of GDF-MRI to standard MRI, from an AUC of 0.75 for the first 50 patients to 0.94 for the final 23 patients. The breast imaging specialized radiologist who had no experience with GDF-MRI also improved in his performance for MR nodal staging using GDF-MRI and after feedback of his errors although his improvement was not statistically significant. This suggests that a learning curve exists.

In this study, two radiologists with different levels of experience in breast cancer imaging and nodal staging participated. The diagnostic performances of standard MRI for both expert (reader 2) and non-expert (reader 1 ) in breast imaging are in line with previous reports on nodal staging in breast cancer with standard T2W MR sequences [20]. This suggests that a radiologists - whether or not specialized in breast cancer imaging, seems to do well with standard T2W MRI.

Of interest is that the performance in nodal staging using the combined standard MRI + GDF-MRI of the two radiologists diverged after feedback of the results of the first 50 patients of the study cohort. In the final 23 patients of our patient cohort, reader 1 showed a significant improvement after addition of GDF-MRI to standard MRI, from an AUC of 0.75 in the first 50 cases to 0.94 in the final 23 cases, equal to the reported figures of rectal cancer $M R$ nodal staging using gadofosveset-enhanced $M R I^{21,22}$. Reader 2 however - despite the fact that he is an expert in breast imaging and knowledgeable of the pitfalls in nodal imaging in breast cancer - only slightly improved in performance after addition of GDF-MRI from 0.75 AUC in the first 50 cases to 0.80 in the final 23 cases. How can it be explained? Reader 1 was inexperienced in breast cancer management and breast imaging interpretation, but had extensive experience in rectal cancer nodal staging with MRI, including 6 years of interpreting gadofosvesetenhanced MRIs. Reader 1 was hence more aware of interpretation difficulties with GDF-MRI and of own pitfalls, because of his previous experience and continuous feedback in rectal cancer ${ }^{21}$. Although reader 2 is an expert breast radiologist, fully knowledgeable of interpretation difficulties in breast cancer axillary ultrasound and MR 
nodal staging and familiar with all consequences of his imaging findings, at the onset of the study he was not familiar at all with GDF-MRI, in contrast to reader 1.

How then can we explain the fact that the GDF MR expert did not do that well in the first 50 patients? This reader started with an advantage of 6 years experience in interpreting lymph nodes on GDF-MRI, nevertheless it took him 50 breast cancer patients' scans, before he reached the accuracy level reliable for clinical decisionmaking. At feedback it became obvious that his main errors were erroneous interpretation of metastatic nodes that resembled reactive nodes. The presence of a fatty hilum was a pitfall and the cause of many false negatives with his readings (see Figure 7.2).

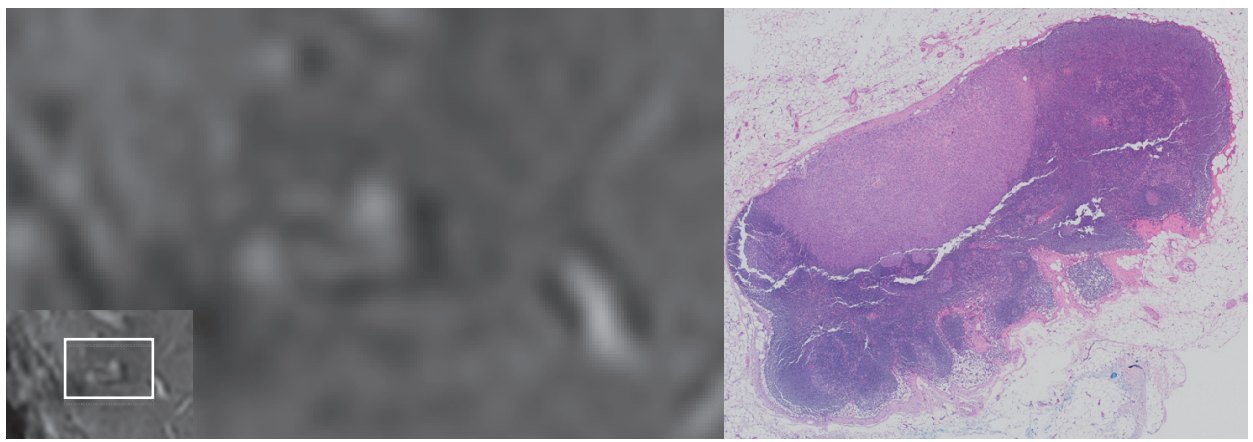

Figure 7.2 An example of a lymph node on a gadofosveset-enhanced axillary MRI. This node was scored as benign for both reader, based on the presence of a chemical shift artifact and a fatty hilum. However, as seen on the H\&E coupe on the right, the dark area of the lymph node was not a fatty hilum, but was mimicked by a metastatic area.

These errors were hardly observed with reader 2 who was more familiar with the spectrum of images of involved breast cancer nodes. However reader 2 was less aware of pitfalls in interpreting MR gadofosveset nodal features, explaining why his level of performance in the final 23 patients remained inferior to reader 1.

The previous promising results of gadofosveset-enhanced MRI for noninvasive axillary nodal staging in breast cancer is again shown but only with a reader with longstanding experience in GDF-MRI nodal staging. The peak of the learning curve for a breast radiologist inexperienced in GDF-MRI lies probably beyond 70 cases. The learning curve could may be reduced in length in in case of continuous feedback, as suggest by other learning curve studies. This is in contrast with the single feedback moment in our study after 50 cases.

The diagnostic performance of standard MRI and standard MRI + GDF-MRI was calculated based on histopathology of the surgically removed lymph nodes. Lymph 
nodes containing ITCS and micrometastases were recorded as nonmalignant findings. Although the number of micrometastases was relatively small in the current study (16/237 lymph nodes), ignoring these small nodal metastases influences both NPV and PPV of axillary MRI. Correctly identified micrometastases would render the nodes false positive, thereby decreasing PPV. At the same time, the NPV increases when ignoring micrometastases. The clinical relevance of these small metastases is under debate and the impact on adjuvant systemic therapy recommendations limited ${ }^{23-28}$. Therefore, ignoring micrometastases and ITCs is justified.

An axillary ultrasound is performed in many countries in addition to physical examination of the axilla for preoperative nodal staging in breast cancer. Axillary ultrasound combined with ultrasound-guided biopsy if indicated, shows high specificity (98.3\%) and high PPV $(97.1 \%)^{29}$. The PPV of GDF-MRI cannot compete with that of ultrasound-guided biopsy, as it is not higher than $83 \%$. Therefore our study suggests that GDF-MRI cannot replace ultrasound-guided nodal biopsy to confirm positive lymph nodes.

Of special interest is the sensitivity of $83 \%$ and NPV of $94 \%$ of GDF-MRI, suggesting that the GDF-MRI is able to identify the (y)pNO patients. This NPV of GDF-MRI outperforms the NPV of axillary ultrasound ${ }^{29-31}$. Furthermore, an NPV of $94 \%$ approximates the NPV of $97 \%$ of the generally accepted but more invasive SLNB ${ }^{32}$. It also outperforms the diagnostic performance of SLNB post-neoadjuvant systemic therapy or the recently proposed MARI-procedure in clinically node positive breast cancer patients ${ }^{33-35}$.

Further outcome studies are needed to understand whether an NPV of $94 \%$ is oncologically safe to replace the current SLNB and ALND. The slightly lower NPV could theoretically increase the risk of regional recurrences, yet this disadvantage could balance the advantage of avoiding treatment related morbidity associated with SLNB and ALND. Avoiding SLNB in cNO patients, and ALND in $\mathrm{cN}+$ patients who converted to ycNO to neoadjuvant systemic therapy, would improve quality of life to a great extent $^{36-38}$.

A limitation of this study is the relatively small number of patients included. This is expressed by the wide $95 \% \mathrm{Cls}$ regarding diagnostic parameters presented on the last one-third of our cohort. Nonetheless, it was our intention to confirm the diagnostic performance of this relative novel imaging tool on a node-by-node basis, as a node-bynode analysis is considered the first essential step to take when introducing a new imaging tool. The diagnostic performance on patient level needs to be further confirmed in a larger group of breast cancer patients, preferably in multicenter studies.

The current study confirmed previous study that GDF-MRI has potential as a noninvasive method for nodal (re-)staging in breast cancer, however a learning curve exists for breast radiologists who are not familiar with GDF-MRI. Before solid 
conclusions can be drawn its diagnostic performance on patient level needs to be confirmed in a larger group of breast cancer patients preferably in a multi-center setting and the value in clinical practice understood by incorporating this method in clinical outcome trials. If these trials will confirm the value of GDF- MRI before broad implementation in clinical practice, we need to invest in training of breast imaging specialized and non-specialized radiologists. 


\section{References}

1. Jemal A, Bray F, Center MM, Ferlay J, Ward E, Forman D. Global cancer statistics. CA Cancer J Clin 2011;61:69-90.

2. Howlader N, Noone AM, Krapcho M, Garshell J, Neyman N, Altekruse SF, Kosary CL, Yu M, Ruhl J, Tatalovich Z, Cho H, Mariotto A, Lewis DR, Chen HS, Feuer EJ, Cronin KA (eds). SEER Cancer Statistics Review, 1975-2010. 2013 April 2013 [cited 2014 02-05]; Available from: http://seer.cancer.gov/ csr/1975_2010/.

3. Sackey H, Magnuson A, Sandelin K, Liljegren G, Bergkvist L, Fulep Z, Celebioglu F, Frisell J. Arm lymphoedema after axillary surgery in women with invasive breast cancer. Br J Surg 2014;101:390-7.

4. Schijven MP, Vingerhoets AJ, Rutten HJ, Nieuwenhuijzen GA, Roumen RM, van Bussel ME, Voogd AC. Comparison of morbidity between axillary lymph node dissection and sentinel node biopsy. Eur J Surg Oncol 2003;29:341-50.

5. Ashikaga T, Krag DN, Land SR, Julian TB, Anderson SJ, Brown AM, Skelly JM, Harlow SP, Weaver DL, Mamounas EP, Costantino JP, Wolmark N. Morbidity results from the NSABP B-32 trial comparing sentinel lymph node dissection versus axillary dissection. J Surg Oncol 2010;102:111-8.

6. Krag DN, Anderson SJ, Julian TB, Brown AM, Harlow SP, Costantino JP, Ashikaga T, Weaver DL, Mamounas EP, Jalovec LM, Frazier TG, Noyes RD, Robidoux A, Scarth HM, Wolmark N. Sentinel-lymphnode resection compared with conventional axillary-lymph-node dissection in clinically node-negative patients with breast cancer: overall survival findings from the NSABP B-32 randomised phase 3 trial. Lancet Oncol 2010;11:927-33.

7. DiSipio T, Rye S, Newman B, Hayes S. Incidence of unilateral arm lymphoedema after breast cancer: a systematic review and meta-analysis. Lancet Oncol 2013;14:500-15.

8. Lucci A, McCall LM, Beitsch PD, Whitworth PW, Reintgen DS, Blumencranz PW, Leitch AM, Saha S, Hunt KK, Giuliano AE; American College of Surgeons Oncology Group. American College of Surgeons Oncology, Surgical complications associated with sentinel lymph node dissection (SLND) plus axillary lymph node dissection compared with SLND alone in the American College of Surgeons Oncology Group Trial Z0011. J Clin Oncol 2007;25:3657-63.

9. Voogd AC, Coebergh JW, Repelaer van Driel OJ, Roumen RM, van Beek MW, Vreugdenhil A, Crommelin MA. The risk of nodal metastases in breast cancer patients with clinically negative lymph nodes: a population-based analysis. Breast Cancer Res Treat 2000;62:63-9.

10. Alvarado R, Yi M, Le-Petross H, Gilcrease M, Mittendorf EA, Bedrosian I, Hwang RF, Caudle AS, Babiera GV, Akins JS, Kuerer HM, Hunt KK. The role for sentinel lymph node dissection after neoadjuvant chemotherapy in patients who present with node-positive breast cancer. Ann Surg Oncol 2012;19:3177-84.

11. Koolen BB, Valdes Olmos RA, Wesseling J, Vogel WV, Vincent AD, Gilhuijs KG, Rodenhuis S, Rutgers EJ, Vrancken Peeters MJ. Early assessment of axillary response with (1)(8)F-FDG PET/CT during neoadjuvant chemotherapy in stage II-III breast cancer: implications for surgical management of the axilla. Ann Surg Oncol 2013;20:2227-35.

12. Rouzier R, Extra JM, Klijanienko J, Falcou MC, Asselain B, Vincent-Salomon A, Vielh P, Bourstyn E. Incidence and prognostic significance of complete axillary downstaging after primary chemotherapy in breast cancer patients with T1 to T3 tumors and cytologically proven axillary metastatic lymph nodes. J Clin Oncol 2002;20:1304-10.

13. Straver ME, Rutgers EJ, Russell NS, Oldenburg HS, Rodenhuis S, Wesseling J, Vincent A, Peeters MT. Towards rational axillary treatment in relation to neoadjuvant therapy in breast cancer. Eur J Cancer 2009;45:2284-92.

14. Schipper RJ, Smidt ML, van Roozendaal LM, Castro CJ, de Vries B, Heuts EM, Keymeulen KB, Wildberger $J E$, Lobbes MB, Beets-Tan RG. Noninvasive nodal staging in patients with breast cancer using gadofosveset-enhanced magnetic resonance imaging: a feasibility study. Invest Radiol 2013;48:134-9.

15. Baltzer PA, Dietzel M, Burmeister HP, Zoubi R, Gajda M, Camara O, Kaiser WA. Application of MR mammography beyond local staging: is there a potential to accurately assess axillary lymph nodes? evaluation of an extended protocol in an initial prospective study. AJR Am J Roentgenol 2011;196:W641-7. 
16. Lambregts DM, Heijnen LA, Maas M, Rutten IJ, Martens MH, Backes WH, Riedl RG, Bakers FC, Cappendijk VC, Beets GL, Beets-Tan RG. Gadofosveset-enhanced MRI for the assessment of rectal cancer lymph nodes: predictive criteria. Abdom Imaging 2013;38:720-7.

17. Lester SC, Bose S, Chen YY, Connolly JL, de Baca ME, Fitzgibbons PL, Hayes DF, Kleer C, O'Malley FP, Page DL, Smith BL, Tan LK, Weaver DL, Winer E; Members of the Cancer Committee, College of American Pathologists. Protocol for the examination of specimens from patients with invasive carcinoma of the breast. Arch Pathol Lab Med 2009;133:1515-38.

18. Efron B. Bootstrap Methods: Another Look at the Jackknife. Ann Statist 1979;7:1-26.

19. DeLong ER, DeLong DM, Clarke-Pearson DL. Comparing the areas under two or more correlated receiver operating characteristic curves: a nonparametric approach. Biometrics 1988;44:837-45.

20. Scaranelo AM, Eiada R, Jacks LM, Kulkarni SR, Crystal P. Accuracy of unenhanced MR imaging in the detection of axillary lymph node metastasis: study of reproducibility and reliability. Radiology 2012; 262:425-34.

21. Lambregts DM, Beets GL, Maas M, Kessels AG, Bakers FC, Cappendijk VC, Engelen SM, Lahaye MJ, de Bruine AP, Lammering G, Leiner T, Verwoerd JL, Wildberger JE, Beets-Tan RG. Accuracy of gadofosveset-enhanced MRI for nodal staging and restaging in rectal cancer. Ann Surg 2011;253: 539-45.

22. Heijnen LA, Lambregts DM, Martens MH, Maas M, Bakers FC, Cappendijk VC, Oliveira P, Lammering G, Riedl RG, Beets GL, Beets-Tan RG. Performance of gadofosveset-enhanced MRI for staging rectal cancer nodes: can the initial promising results be reproduced? Eur Radiol 2014;24:371-9.

23. Giuliano AE, Hawes D, Ballman KV, Whitworth PW, Blumencranz PW, Reintgen DS, Morrow M, Leitch AM, Hunt KK, McCall LM, Abati A, Cote R. Association of occult metastases in sentinel lymph nodes and bone marrow with survival among women with early-stage invasive breast cancer. JAMA 2011;306: 385-93.

24. Gobardhan PD, Elias SG, Madsen EV, van Wely B, van den Wildenberg F, Theunissen EB, Ernst MF, Kokke MC, van der Pol C, Borel Rinkes IH, Wijsman JH, Bongers V, van Gorp J, van Dalen T. Prognostic value of lymph node micrometastases in breast cancer: a multicenter cohort study. Ann Surg Oncol 2011;18:1657-64.

25. Maaskant-Braat AJ, van de Poll-Franse LV, Voogd AC, Coebergh JW, Roumen RM, Nolthenius-Puylaert MC, Nieuwenhuijzen GA. Sentinel node micrometastases in breast cancer do not affect prognosis: a population-based study. Breast Cancer Res Treat 2011;127:195-203.

26. van Roozendaal LM, Schipper RJ, Van de Vijver KK, Haekens CM, Lobbes MB, Tjan-Heijnen VC, de Boer M, Smidt ML. The impact of the pathological lymph node status on adjuvant systemic treatment recommendations in clinically node negative breast cancer patients. Breast Cancer Res Treat 2014; 143:469-76.

27. Weaver DL, Ashikaga T, Krag DN, Skelly JM, Anderson SJ, Harlow SP, Julian TB, Mamounas EP, Wolmark N. Effect of occult metastases on survival in node-negative breast cancer. N Engl J Med 2011;364: 412-21.

28. de Boer M, van Deurzen CH, van Dijck JA, Borm GF, van Diest PJ, Adang EM, Nortier JW, Rutgers EJ, Seynaeve C, Menke-Pluymers MB, Bult P, Tjan-Heijnen VC. Micrometastases or isolated tumor cells and the outcome of breast cancer. N Engl J Med 2009;361:653-63.

29. Houssami N, Ciatto S, Turner RM, Cody HS 3rd, Macaskill P. Preoperative ultrasound-guided needle biopsy of axillary nodes in invasive breast cancer: meta-analysis of its accuracy and utility in staging the axilla. Ann Surg 2011;254:243-51.

30. Schipper RJ, van Roozendaal LM, de Vries B, Pijnappel RM, Beets-Tan RG, Lobbes MB, Smidt ML. Axillary ultrasound for preoperative nodal staging in breast cancer patients: is it of added value? Breast 2013; 22:1108-13.

31. Alvarez S, Anorbe E, Alcorta P, Lopez F, Alonso I, Cortes J. Role of sonography in the diagnosis of axillary lymph node metastases in breast cancer: a systematic review. AJR Am J Roentgenol 2006;186:1342-8.

32. Pesek S, Ashikaga T, Krag LE, Krag D, The false-negative rate of sentinel node biopsy in patients with breast cancer: a meta-analysis. World J Surg 2012;36:2239-51. 
33. Boughey JC, Suman VJ, Mittendorf EA, Ahrendt GM, Wilke LG, Taback B, Leitch AM, Kuerer HM, Bowling M, Flippo-Morton TS, Byrd DR, Ollila DW, Julian TB, McLaughlin SA, McCall L, Symmans WF, Le-Petross HT, Haffty BG, Buchholz TA, Nelson H, Hunt KK; Alliance for Clinical Trials in Oncology. Sentinel lymph node surgery after neoadjuvant chemotherapy in patients with node-positive breast cancer: the ACOSOG Z1071 (Alliance) clinical trial. JAMA, 2013;310:1455-61.

34. Kuehn T, Bauerfeind I, Fehm T, Fleige B, Hausschild M, Helms G, Lebeau A, Liedtke C, von Minckwitz G, Nekljudova V, Schmatloch S, Schrenk P, Staebler A, Untch M. Sentinel-lymph-node biopsy in patients with breast cancer before and after neoadjuvant chemotherapy (SENTINA): a prospective, multicentre cohort study. Lancet Oncol, 2013;14:609-18.

35. Donker M, Straver ME, Wesseling J, Loo CE, Schot M, Drukker CA, van Tinteren H, Sonke GS, Rutgers EJ, Vrancken Peeters MJ. Marking Axillary Lymph Nodes With Radioactive lodine Seeds for Axillary Staging After Neoadjuvant Systemic Treatment in Breast Cancer Patients: The MARI Procedure. Ann Surg, 2014.

36. Heiney SP, McWayne J, Cunningham JE, Hazlett L, Parrish RS, Bryant LH, Vitoc C, Jansen K. Quality of life and lymphedema following breast cancer. Lymphology, 2007;40:177-84.

37. Pyszel A, Malyszczak K, Pyszel K, Andrzejak R, Szuba A. Disability, psychological distress and quality of life in breast cancer survivors with arm lymphedema. Lymphology, 2006;39:185-92.

38. Shih YC, Xu Y, Cormier JN, Giordano S, Ridner SH, Buchholz TA, Perkins GH, Elting LS. Incidence, treatment costs, and complications of lymphedema after breast cancer among women of working age: a 2-year follow-up study. J Clin Oncol, 2009;27:2007-14. 


\section{Chapter}

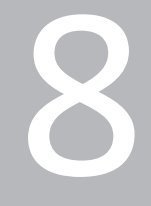

The impact of the pathological lymph node status on adjuvant systemic treatment recommendations in clinically node negative breast cancer patients

L.M. van Roozendaal*

RJ. Schipper*

K.K.B.T. Van de Vijver

C.M. Haekens

M.B.I. Lobbes

V.C.G. Tjan-Heijnen

M. de Boer

M.L. Smidt

* Both authors contributed equally to this manuscript

Breast Cancer Res Treat 2014;143:469-76 


\section{Abstract}

\section{Background}

Several independent randomized controlled trials are initiated to investigate whether sentinel lymph node biopsy can be safely omitted in clinically node negative breast cancer patients with negative axillary ultrasound findings, who are treated with breast conserving therapy. A consequence of omitting sentinel lymph node biopsy is absence of pathological lymph node status information. We aimed to investigate the impact of omitting sentinel lymph node biopsy on adjuvant systemic treatment recommendations.

\section{Materials and methods}

Data from all consecutive patients with invasive breast cancer and negative axillary ultrasound findings treated with breast conserving therapy and sentinel lymph node biopsy between 2008 and 2012 were collected from a prospective database. Two methods, Adjuvant! Online and the Dutch breast cancer guideline 2012, were used to determine the adjuvant systemic treatment recommendations of every patient. At first, each patient was considered to be lymph node negative, and secondly the patients' true pathological lymph node status was used.

\section{Results}

A total of 303 patients were consecutively included. Pathological lymph node status was pNO in $72.3 \%, \mathrm{pNO}(\mathrm{i}+$ ) in $12.9 \%, \mathrm{pN} 1 \mathrm{mi}+$ in $5.6 \%, \mathrm{pN} 1$ in $7.3 \%$, and $\mathrm{pN} 2$ in $2.0 \%$ of the patients. The decision to recommend adjuvant systemic treatment changed due to the pathological lymph node status in $1.0 \%$ of the patients (3/303) when using Adjuvant! Online and in $3.6 \%(11 / 303)$ when using the 2012 Dutch breast cancer guideline.

\section{Conclusion}

The impact of the pathological lymph node status on adjuvant systemic treatment recommendations in clinically node negative breast cancer patients with negative axillary ultrasound findings treated with breast conserving therapy is limited. The safety of omitting the sentinel lymph node biopsy should be confirmed by the initiated randomized controlled trials. 


\section{Introduction}

The presence of lymph node metastases is considered a strong prognostic factor in breast cancer ${ }^{1,2}$. The pathological lymph node status is commonly assessed to select breast cancer patients who might benefit from adjuvant systemic treatment. For a long period, the axillary lymph node dissection was performed in each patient to identify patients with lymph node metastases. Fifteen years ago, the axillary lymph node dissection was replaced by the sentinel lymph node biopsy (SLNB) in clinically node negative breast cancer patients, followed by a completion axillary lymph node dissection only in patients with a positive sentinel lymph node. As a result, significantly lower arm morbidity rates were observed, and the pathological lymph node status was still assessed adequately ${ }^{3-5}$.

Currently, indications for the performance of a completion axillary lymph node dissection are fading. Several randomized controlled trials demonstrated that either performing axillary radiotherapy ${ }^{6}$, or omitting further axillary treatment ${ }^{7,8}$ following sentinel lymph node metastases did not compromise overall survival, nor regional disease control, while $11-27 \%$ of patients were likely to have residual nodal disease that was not surgically removed. These findings raise doubt about the value of the SLNB itself. Especially, because the risk of (extensive) lymph node positive disease is low in patients treated with breast conserving therapy and in patients with negative physical examination and negative ultrasound findings of the axilla ${ }^{9-12}$. These observations led to the development of several independent randomized controlled trials (e.g. BOOG 2013-08, SOUND, INSEMA, and NCT01821768), that aim to investigate whether the SLNB can be safely omitted in clinically node negative breast cancer patients with negative axillary ultrasound findings, who are treated with breast conserving therapy $^{13,14}$.

A consequence of omitting the SLNB is the absence of pathological lymph node status information, which could influence the decision to recommend adjuvant systemic treatment. Thus, patients could be at risk for systemic undertreatment and a decreased disease-free and overall survival. To simulate the impact of omitting the SLNB on adjuvant systemic treatment recommendations, we performed a study in a cohort of clinically node negative breast cancer patients with negative axillary ultrasound findings, who were treated with breast conserving therapy in our breast clinic.

\section{Materials and methods}

\section{Study population and data collection}

The acquisition of informed consent was waived by the medical ethics committee of Maastricht University Medical Center+. All consecutive patients diagnosed from 2008 to 
2012 with: 1) operable primary invasive breast cancer; 2) primary tumor size $\leq 5 \mathrm{~cm}$; and 3) a clinically node negative status (i.e. negative physical examination and negative axillary ultrasound findings, or negative cyto- or histology) were considered for inclusion. Only patients who were primarily treated with breast conserving therapy (i.e. lumpectomy followed by whole breast radiotherapy) and SLNB in our breast clinic were finally included from a prospective database. Data concerning age; sex; diagnostic work-up; surgical procedures; and pathology reporting of tumor type, grade, size, receptor status and lymph nodes were extracted from the database.

\section{Clinical nodal status}

The axillary ultrasound was performed by dedicated breast radiologists (regardless of the results of physical examination), using an ATL-HDI5000 system, which in 2011 was replaced by an iU22-xMATRIX ultrasound system in combination with a high-frequency linear array transducer (Philips Healthcare, Best, the Netherlands). In our institution, the entire axilla is examined in a standardized fashion, starting at the low axilla (level I), and continuing upwards toward mid-axilla (level II), and apical axilla (level III). Tissue sampling was performed if lymph nodes were suspicious for a metastasis on ultrasound (based on cortical thickening, effacement or replacement of the fatty hilum, nonhilar blood flow and size) using 16-18 gauge core needle biopsy. When core needle biopsy was technically challenging, for example due to the proximity of a large blood vessel, fine needle aspiration cytology was used.

\section{Surgical techniques}

The sentinel lymph node was identified by using a triple technique consisting of lymphoscintigraphy (using $80 \mathrm{MBq}$ Technetium-99m nanocolloid injected periareolar), blue dye to detect lymphatic vessels (Bleu Patente V; Guerbet, Aulnay-sous-Bois, France), and a gamma probe to detect radioactivity. After the SLNB, palpation of the axilla was performed to identify and consequently remove suspicious non-sentinel lymph nodes. In all cases with one or more (micro-)metastasis in the sentinel lymph node, a completion axillary lymph node dissection was performed.

\section{Pathological techniques}

Sentinel lymph nodes were sliced with a maximum thickness of $3 \mathrm{~mm}$ and embedded in paraffin after formalin fixation. Each paraffin block was step sectioned at 500-micrometer intervals at three levels and stained with hematoxylin and eosin. If no metastasis was detected with hematoxylin and eosin, immunohistochemical staining was performed with a mouse anti-human MNF116 antibody (Dako).

All lymph nodes of the completion axillary lymph node dissection specimen were embedded in paraffin after formalin fixation. Lymph nodes larger than $5 \mathrm{~mm}$ were 
sliced with a maximum thickness of $3 \mathrm{~mm}$. All slides were stained with hematoxylin and eosin. Each node was recorded as either benign (pNO) or as an isolated tumor cell $(\mathrm{pNO}(\mathrm{i}+))(\leq 0.2 \mathrm{~mm})$, micrometastasis $(\mathrm{pN} 1 \mathrm{mi})(0.2 \leq 2.0 \mathrm{~mm})$ or macrometastasis $(\mathrm{pN} 1-3)$ $(>2.0 \mathrm{~mm})$.

Tumors were graded according to the modified Bloom-Richardson grading system. The estrogen receptor status and the progesterone receptor status was determined by immunohistochemistry and scored using $<10 \%$ of tumor staining as the negative cut off. Fluorescence in situ hybridization analyses was used to detect amplification of HER2/neu with a cut off ratio of $\geq 2$.0. All in accordance to the 2012 Dutch breast cancer guideline $^{15}$.

\section{Adjuvant systemic treatment recommendations}

The decision to recommend adjuvant systemic treatment (i.e.: adjuvant hormonaland/or chemotherapy and/or trastuzumab) for breast cancer patients is based on local breast cancer guidelines and normally discussed by a multidisciplinary tumor board. However, to obtain objective adjuvant systemic treatment recommendations, we applied Adjuvant! Online as well as the 2012 Dutch breast cancer guideline in our study cohort.

Adjuvant! Online, version 8.0, is an online decision making tool that clinicians can use to calculate the ten year survival probability of a breast cancer patient. This probability is based on patient's age, co-morbidities, pathological tumor size, tumor grade, ER-status and number of lymph node metastases. Patients are considered as having low risk when the ten year survival probability is more than $92 \%$, based on consensus among the TRANSBIG Consortium members (www.breastinternationalgroup.org) ${ }^{6}$.

The 2012 Dutch breast cancer guideline describes that adjuvant systemic treatment is recommended for patients with an absolute ten year mortality risk $\geq 15 \%$. This concept is translated into the following guideline: adjuvant systemic treatment is recommended for patients with a primary tumor size $\mathbf{} \mathbf{2 . 0} \mathrm{cm}$; for patients with a primary tumor size between $1.0 \mathrm{~cm}$ and $2.0 \mathrm{~cm}$, in case of a tumor grade 2 or 3 (modified BloomRichardson grading system); for patients with a primary tumor size $\geq 0.5 \mathrm{~cm}$, in case of HER2/neu amplification; for all patients aged $<35$ years, excluding patients with a primary tumor size $<1.0 \mathrm{~cm}$ and a tumor grade 1 (modified Bloom-Richardson); and for all patients with a pathological lymph node positive status (including patients with a micrometastasis). Furthermore, adjuvant chemotherapy (with or without trastuzumab) can be considered for fit patients aged 70 years or older with a pathological lymph node positive status and hormone receptor negative breast cancer.

Adjuvant! Online and the 2012 Dutch breast cancer guideline use different cut off points to classify a patient as having low or high risk breast cancer, and consequently the recommendation to administer adjuvant systemic treatment. In other words, when comparing both methods, Adjuvant! Online is more "aggressive" and the 2012 Dutch 
breast cancer guideline is more "restricted" in recommending adjuvant systemic treatment.

For each patient in our study cohort we calculated the ten year survival probability in Adjuvant! Online. At first, each patient was considered to have a negative lymph node status, and secondly the patients' true pathological lymph node status was used. The lymph node status in Adjuvant! Online could be scored as 'node negative', as '1-3 positive nodes', as '4-9 positive nodes', or as '10 or more positive nodes'. Patients with only isolated tumor cells were categorized as node negative and patients with micrometastases were categorized as having 1-3 positive nodes. The co-morbidity rate was classified as low for all patients. Adjuvant systemic treatment was recommended in patients classified as high risk (10 year survival probability lower than and including $92 \%$ ), and not recommended in patients classified as low risk (10 year survival probability more than $92 \%$ ).

In addition, adjuvant systemic treatment recommendations were determined for each patient according to the 2012 Dutch breast cancer guideline. Again, first each patient was considered to have a negative lymph node status, and secondly the patients' true pathological lymph node status was used.

Cases in which the decision changed from adjuvant systemic treatment not recommended (when using a lymph node negative status) to adjuvant systemic treatment recommended (when using patients' true pathological lymph node status) were considered to be conflicting outcomes.

\section{Statistical analyses}

Descriptive categorical data are presented as proportions and absolute numbers. Continuous variables are presented as means with standard deviations. Statistical analyses were performed using Statistical Package for the Social Sciences (SPSS, version 19.0, Chicago, Illinois, USA).

\section{Results}

\section{Patient demographics and primary tumor characteristics}

In the study period, 660 patients primarily underwent surgery for invasive breast cancer in our breast clinic. After applying the inclusion criteria, 303 patients were eligible for final analysis in this study (Figure 8.1). Of these, patient and tumor characteristics are summarized in Table 8.1. Mean age was 60 years (SD 10, range 30-87) and mean tumor size was $15 \mathrm{~mm}$ (SD 7, range 2-48). Invasive ductal carcinoma, not otherwise specified, was the most common type of breast cancer in $82.8 \%$, followed by invasive lobular carcinoma in $9.9 \%$, and other types of breast cancer in $7.3 \%$ of the patients. Estrogen 
receptor positive breast cancer was detected in $83.8 \%$, and HER2/neu was amplified in $11.2 \%$.

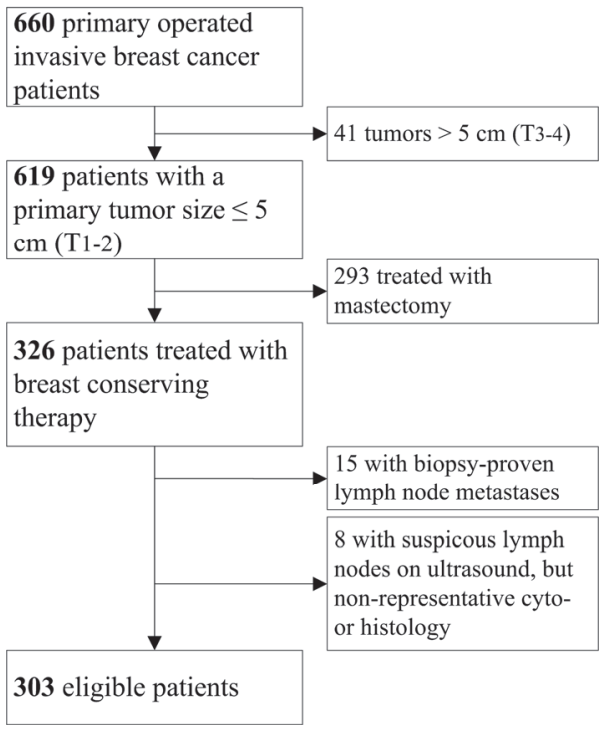

Figure 8.1 Flowdiagram of inclusion and exclusion of patients.

\section{Pathological lymph node status}

The mean number of (non-)sentinel lymph nodes removed during the SLNB was 2 (SD 1, range 1-7) (Table 8.1). The sentinel lymph nodes contained no metastatic disease in 219 patients (72.3\%), isolated tumor cells in 39 (12.9\%), micrometastases in 17 (5.6\%), and one or more macrometastases in 28 patients (9.2\%). Subsequently, a completion axillary lymph node dissection was performed in the 45 sentinel lymph node positive patients, resulting in a mean number of 17 removed lymph nodes including the sentinel lymph nodes (SD 7, range 6-38). In 34 of the 45 patients (75.6\%), no additional lymph node metastases were detected. Additional isolated tumor cells were detected in one (2.2\%), micrometastases in two (4.4\%), and macrometastases in eight patients (17.8\%). Final pathological lymph nodes status was pNO in 219 (72.3\%), pNO(i+) in 39 (12.9\%), pN1mi in 17 (5.6\%), pN1 in $22(7.3 \%)$, and pN2 in 6 patients (2.0\%). No patients were staged as pN3. 
Table 8.1 Patient demographics and tumor characteristics.

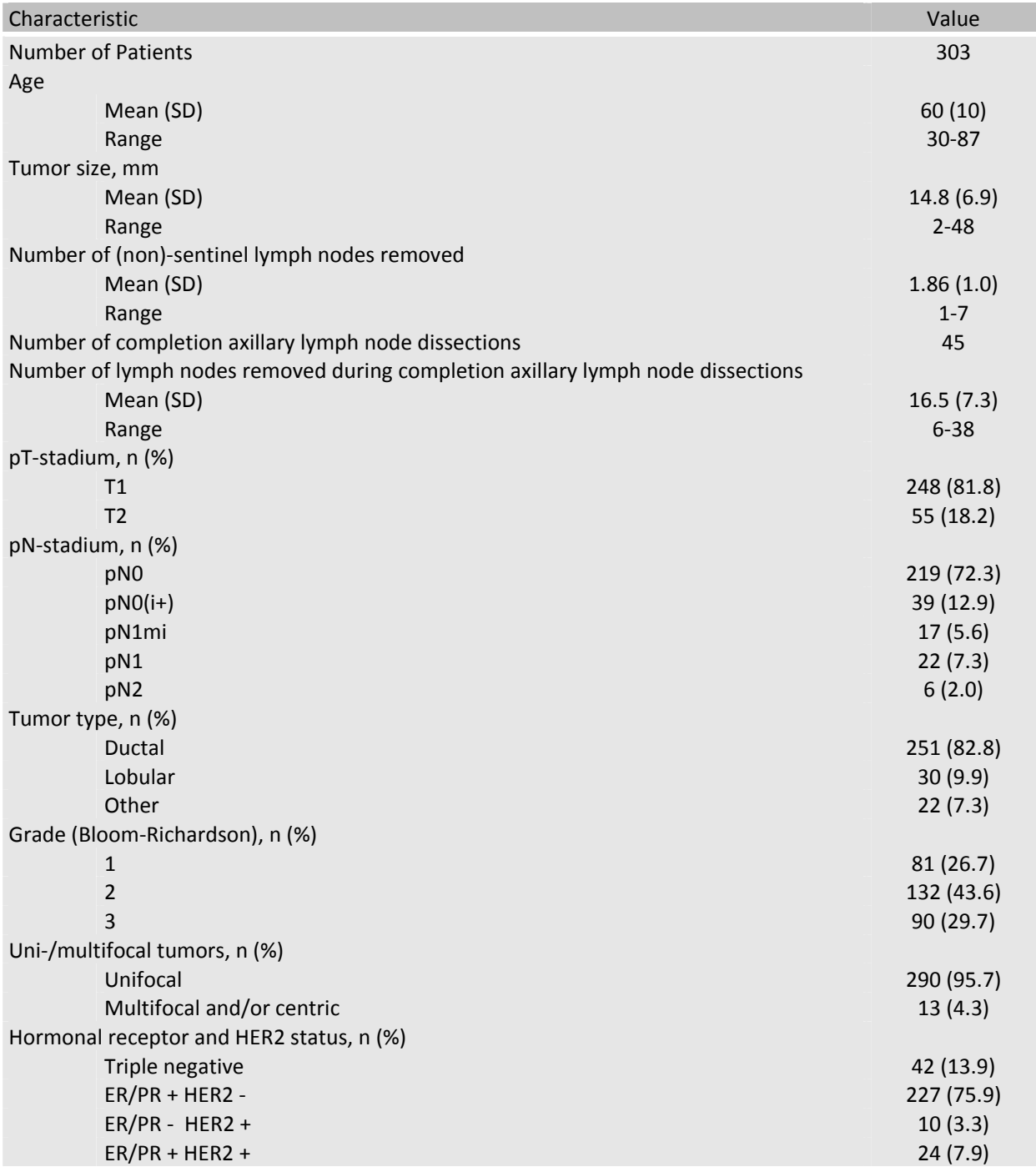

$\mathrm{N}$ : number of cases; SD: standard deviation; ER: estrogen receptor; PR: progesterone receptor; HER2: human epidermal growth factor receptor 2 .

\section{Adjuvant systemic treatment recommendations - Adjuvant! Online}

When patients' lymph node status was considered as negative, a total of 268 patients (88.4\%) were classified as high risk and 35 patients (11.6\%) as low risk. When patients' true pathological lymph node status was applied, an additional three patients were considered as high risk. Thus, based on the risk estimation by Adjuvant! Online, the 
decision to recommend adjuvant systemic treatment changed for $3 / 303$ patients (1.0\%).

\section{Adjuvant systemic treatment recommendations -The 2012 Dutch breast cancer guideline}

When patients' lymph node status was considered as negative, a total of 189 (61.5\%) patients were classified as high risk and 114 (38.5\%) patients as low risk. When patients' true pathological lymph node status was applied, an additional 11 patients were considered as high risk. Thus, based on the 2012 Dutch breast cancer guideline, the decision to recommend adjuvant systemic treatment changed in $11 / 303$ patients (3.6\%).

\section{Conflicting outcomes}

The three patients with conflicting outcomes based on Adjuvant! Online also had conflicting outcomes when the 2012 Dutch breast cancer guideline was applied. So, in a total of 11 patients the decision changed from adjuvant systemic treatment not recommended (when using a lymph node negative status) to adjuvant systemic treatment recommended (when using patients' true pathological nodal status), based on Adjuvant! Online and/or the 2012 Dutch breast cancer guideline. Demographics and tumor characteristics of these patients are reported in Table 8.2.

Table 8.2 Demographics and tumor characteristics of patients with conflicting outcomes regarding adjuvant systemic treatment recommendation.

\begin{tabular}{lccccccccccc} 
Age & \multicolumn{1}{c}{$\begin{array}{c}\text { Size } \\
\text { tumor } \\
(\mathrm{mm})\end{array}$} & Grade & $\begin{array}{c}\text { Nodal } \\
\text { status }\end{array}$ & ER & PR & HER2 & LVI & $\begin{array}{c}\text { Adjuvant! Online } \\
\text { - conflicting } \\
\text { outcome }\end{array}$ & $\begin{array}{c}\text { Dutch Guideline } \\
\text { - conflicting } \\
\text { outcome }\end{array}$ \\
\hline 1 & 74 & IDC & 15 & 1 & pN1mi & Yes & Yes & No & No & No & Yes \\
2 & 60 & IDC & 9 & 1 & pN1mi & Yes & No & No & No & No & Yes \\
3 & 58 & IDC & 4 & 1 & pN1 & Yes & Yes & No & No & No & Yes \\
4 & 66 & IDC & 2 & 2 & pN2 & Yes & No & No & Yes & No & Yes \\
5 & 60 & IDC & 8 & 2 & pN1mi & Yes & Yes & No & Yes & No & Yes \\
6 & 48 & IDC & 9 & 2 & pN1 & Yes & Yes & No & Yes & Yes & Yes \\
7 & 44 & IDC & 18 & 1 & pN1mi & Yes & Yes & No & Yes & No & Yes \\
8 & 66 & IDC & 11 & 1 & pN1mi & Yes & Yes & No & No & No & Yes \\
9 & 62 & IDC & 19 & 1 & pN2 & Yes & Yes & No & No & No & Yes \\
10 & 49 & IDC & 12 & 1 & pN2 & Yes & Yes & No & Yes & Yes & Yes \\
11 & 54 & IDC & 9 & 1 & pN1mi & Yes & Yes & No & No & Yes & Yes
\end{tabular}

IDC: invasive ducal carcinoma; Grade: according to modified Bloom-Richardson; ER: estrogen receptor; PR: progesterone receptor; HER2: human epidermal growth factor receptor 2; LVI: lymphovascular invasion. 


\section{Discussion}

This study simulated the impact of omitting the SLNB on adjuvant treatment recommendations in clinically node negative breast cancer patients with negative axillary ultrasound findings, who were treated with breast conserving therapy. The impact was simulated using both Adjuvant! Online and the 2012 Dutch breast cancer guideline. The decision to recommend adjuvant systemic treatment changed due to the pathological lymph node status in $1.0 \%$ of the patients when using Adjuvant! Online, and in 3.6\% when using the 2012 Dutch breast cancer guideline.

Adjuvant systemic treatment is nowadays not only recommended in patients with lymph node metastases, but also in lymph node negative patients with high risk tumor characteristics. The pathological lymph node status can still be important though, for instance in the presence of solely micrometastases in patients with favorable primary tumor characteristics. In our cohort, one (based on Adjuvant! Online) to six (based on 2012 Dutch breast cancer guideline) patients with conflicting outcomes regarding recommendation of adjuvant systemic treatment were diagnosed with micrometastases, while they were classified as having low risk breast cancer based on their primary tumor characteristics (Table 8.2). Although a micrometastasis in the sentinel lymph node is considered to have an impact on the prognosis of patients with low risk primary breast cancer, the presence of micrometastasis will not elevate the risk in such a way, that the benefit of adjuvant systemic treatment is large enough to justify the standard administration of this treatment ${ }^{9,16-22}$. Ignoring the presence of micrometastatic sentinel lymph nodes would therefore result in fewer conflicting adjuvant treatment recommendations in our study cohort.

Several gene-expression profiles have been developed with the aim to select patients who most likely benefit from adjuvant chemotherapy (with or without trastuzumab), compared to the use of patient and tumor characteristics only ${ }^{23}$. Gene expression profiles are performed on primary tumor material. Therefore, lymph node sampling is not needed. Theoretically, only MammaPrint has the potential to be used in our study cohort, since MammaPrint is developed for patients with lymph node negative and positive (1-3 lymph node metastases) (and estrogen receptor negative and positive) breast cancer. MammaPrint is now being prospectively validated in the randomized controlled MINDACT trial to determine its accuracy ${ }^{24}$. Results of this trial have to be awaited before MammaPrint ${ }^{\circledR}$ can be generally implemented in the decision making regarding adjuvant systemic treatment in breast cancer patients, also in cases where lymph node status is considered negative based on axillary ultrasound.

Previously, the impact of the total number of lymph node metastases on the adjuvant systemic treatment recommendations was questioned after publication of results of 
the randomized controlled AMAROS, ACOSOG Z0011 and IBCSG 23-01 trials ${ }^{6-8}$. These trials have all shown that either performing axillary radiotherapy, or omitting further axillary treatment instead of a completion axillary lymph node dissection following positive sentinel lymph nodes does not compromise overall survival, nor disease control. The administration of adjuvant systemic treatment in these trials did not differ significantly between the experimental and the control arm. It was therefore concluded that incomplete information on the pathological lymph node status did not significantly influence adjuvant systemic treatment recommendations ${ }^{7,25}$.

The minor change in adjuvant systemic treatment recommendations in our cohort might be the result of the inclusion of only patients with low risk breast cancer who were treated with breast conserving therapy, combined with negative axillary ultrasound findings in all patients. The risk of extensive lymph node disease is limited in patients treated with breast conserving therapy ${ }^{9}$. Further, Zgajnar et al. showed that the total number of lymph node metastases per patient was lower after a negative axillary ultrasound compared to when the clinically node negative status was based solely on physical examination ${ }^{10}$. In addition, other studies showed that after a negative axillary ultrasound only $18 \%$ of patients were diagnosed with 1-3 lymph node metastases, and only 4-7\% with four or more lymph node metastases in an unselected breast cancer population ${ }^{11,12}$. In our study cohort, $1.9 \%$ of the patients were finally diagnosed with four or more lymph node metastases, compared to $12.8 \%$ of the patients in a study cohort of Aigner et al. with similar inclusion criteria ${ }^{26}$. This reflects that the accuracy of the axillary ultrasound can be institution and operator dependent. A nomogram was developed to select patients with four or more lymph node metastases ${ }^{27}$. However, results showed that a negative axillary ultrasound exceeds the diagnostic performance of this nomogram, to select patients with extensive nodal disease and thus prevent systemic undertreatment when omitting the SLNB.

We showed that the decision to recommend adjuvant systemic treatment changed by knowledge of the lymph node status in up to $3.6 \%$ in our study cohort. Occult lymph node metastases are not surgically, nor adjuvant systemically treated when the SLNB is omitted in these patients. These patients are therefore at risk of the development of regional recurrence, and/or distant metastases. If adjuvant systemic treatment would truly reduce the risk of developing distant metastases in $20-57 \%$ of these patients ${ }^{28}$, $0.7-2.1 \%$ of patients in our cohort will be systemically undertreated. Nonetheless, all these patients will be treated with whole breast radiotherapy that might eradicate metastatic lymph nodes left in situ, thereby potentially preventing the development of a regional recurrence and subsequently distant metastases ${ }^{29}$. Although speculative, with these results in mind it could be questioned whether such a change in adjuvant systemic treatment recommendations could result in such a significant difference in 
regional control and ultimately overall survival, that justifies the performance of an SLNB and its associated morbidity.

Another risk of omitting the SLNB might be that internal mammary sentinel lymph node metastases are not detected, while its presence could guide radiotherapy planning. In a prospective study on SLNB of Heuts et al., the SLNB was performed by peritumoral injection of $370 \mathrm{MBq}$ Technetium-99m nanocolloid (compared to periareolar injection of $80 \mathrm{MBq}$ in our study). Despite of this extensive SLNB protocol, the study showed that radiotherapy planning changed in only $0.9 \%$ of clinically node negative breast cancer patients ${ }^{30}$. The value of detecting internal mammary (or periclavicular) sentinel lymph nodes might further decrease following results of the EORTC trial 22911/10925, that were recently presented ${ }^{31}$. In this trial, lymph node positive breast cancer patients, or lymph node negative patients with a centrally or medially located primary tumor (treated with mastectomy or breast conserving therapy) were randomized for whether or not to undergo radiotherapy of the internal mammary and medial supraclavicular (IM-MS) lymph nodes. Ten year follow-up results showed a small improvement of overall survival for patients in the IM-MS radiation group versus patients in the control group. However, this benefit is too small to justify IM-MS radiotherapy and subsequently potential complications in every eligible patient. Results of subgroup analyses need to be awaited to be able to select patients who are likely to have great benefit from this treatment, while taking into consideration that an evaluation of HER2/neu amplification was not performed at the time of inclusion.

A limitation of our study is that it is a reflection of a single center experience based on the 2012 Dutch breast cancer guideline and Adjuvant! Online. As national guidelines and physician's preferences can differ with regard to the recommendation of adjuvant systemic treatment, we chose to apply both our national guideline and an international tool, Adjuvant! Online. Physicians can decide whether their local guideline relates more to Adjuvant! Online or to the 2012 Dutch breast cancer guideline. Furthermore, applicability of our study results to other patient populations also depends on whether or not an axillary ultrasound is performed in the work-up of patients with breast cancer.

In conclusion, the impact of the pathological lymph node status on adjuvant systemic treatment recommendations in clinically node negative breast cancer patients with negative axillary ultrasound findings treated with breast conserving therapy is limited. The safety of omitting the SLNB should be confirmed by the initiated randomized controlled trials. 


\section{References}

1. Truong PT, Vinh-Hung V, Cserni G, Woodward WA, Tai P, Vlastos G, Member of the International Nodal Ratio Working $G$. The number of positive nodes and the ratio of positive to excised nodes are significant predictors of survival in women with micrometastatic node-positive breast cancer. Eur J Cancer 2008;44:1670-7.

2. Vinh-Hung V, Verkooijen HM, Fioretta G, Neyroud-Caspar I, Rapiti E, Vlastos G, Deglise C, Usel M, Lutz $\mathrm{JM}$, Bouchardy C. Lymph node ratio as an alternative to $\mathrm{pN}$ staging in node-positive breast cancer. J Clin Oncol 2009;27:1062-8.

3. Ashikaga T, Krag DN, Land SR, Julian TB, Anderson SJ, Brown AM, Skelly JM, Harlow SP, Weaver DL, Mamounas EP, Costantino JP, Wolmark N. Morbidity results from the NSABP B-32 trial comparing sentinel lymph node dissection versus axillary dissection. J Surg Oncol 2010;102:111-8.

4. Krag DN, Anderson SJ, Julian TB, Brown AM, Harlow SP, Costantino JP, Ashikaga T, Weaver DL, Mamounas EP, Jalovec LM, Frazier TG, Noyes RD, Robidoux A, Scarth HM, Wolmark N. Sentinel-lymphnode resection compared with conventional axillary-lymph-node dissection in clinically node-negative patients with breast cancer: overall survival findings from the NSABP B-32 randomised phase 3 trial. Lancet Oncol 2010;11:927-33.

5. Veronesi U, Paganelli G, Galimberti V, Viale G, Zurrida S, Bedoni M, Costa A, de Cicco C, Geraghty JG, Luini A, Sacchini V, Veronesi P. Sentinel-node biopsy to avoid axillary dissection in breast cancer with clinically negative lymph-nodes. Lancet 1997;349:1864-7.

6. Rutgers EJ, Donker M, Straver ME, Meijnen P, Van De Velde CJH, Mansel RE, Westenberg H, Orzalesi L, Bouma WH, van der Mijle H, Nieuwenhuijzen GAP, Veltkamp SC, Slaets L, Messina CGM, Duez NJ, Hurkmans C, Bogaerts J, van Tienhoven G. Radiotherapy or surgery of the axilla after a positive sentinel node in breast cancer patients: Final analysis of the EORTC AMAROS trial (10981/22023). J Clin Oncol 2013;31 (suppl; abstr LBA1001)

7. Giuliano AE, Hunt KK, Ballman KV, Beitsch PD, Whitworth PW, Blumencranz PW, Leitch AM, Saha S, McCall LM, Morrow M. Axillary dissection vs no axillary dissection in women with invasive breast cancer and sentinel node metastasis: a randomized clinical trial. JAMA 2011;305:569-75.

8. Galimberti V, Cole BF, Zurrida S, Viale G, Luini A, Veronesi P, Baratella P, Chifu C, Sargenti M, Intra M, Gentilini O, Mastropasqua MG, Mazzarol G, Massarut S, Garbay JR, Zgajnar J, Galatius H, Recalcati A, Littlejohn D, Bamert M, Colleoni M, Price KN, Regan MM, Goldhirsch A, Coates AS, Gelber RD, Veronesi $U$, International Breast Cancer Study Group Trial i. Axillary dissection versus no axillary dissection in patients with sentinel-node micrometastases (IBCSG 23-01): a phase 3 randomised controlled trial. Lancet Oncol 2013;14:297-305.

9. Maaskant-Braat AJ, van de Poll-Franse LV, Voogd AC, Coebergh JW, Roumen RM, Nolthenius-Puylaert MC, Nieuwenhuijzen GA. Sentinel node micrometastases in breast cancer do not affect prognosis: a population-based study. Breast Cancer Res Treat 2011;127:195-203.

10. Zgajnar J, Hocevar M, Podkrajsek M, Hertl K, Frkovic-Grazio S, Vidmar G, Besic N. Patients with preoperatively ultrasonically uninvolved axillary lymph nodes: a distinct subgroup of early breast cancer patients. Breast Cancer Res Treat 2006;97:293-9.

11. Neal CH, Daly CP, Nees AV, Helvie MA. Can preoperative axillary US help exclude N2 and N3 metastatic breast cancer? Radiology 2010;257:335-41.

12. Schipper RJ, van Roozendaal LM, de Vries B, Pijnappel RM, Beets-Tan RG, Lobbes MB, Smidt ML. Axillary ultrasound for preoperative nodal staging in breast cancer patients: Is it of added value? Breast 2013;22:1108-13.

13. Gentilini O, Veronesi U. Abandoning sentinel lymph node biopsy in early breast cancer? A new trial in progress at the European Institute of Oncology of Milan (SOUND: Sentinel node vs Observation after axillary UltraSouND). Breast 2012;21:678-81.

14. van Roozendaal LM, de Wilt JHW, Smidt ML. Clinical node negative breast cancer patients undergoing breast conserving therapy: follow-up versus sentinel lymph node biopsy. Paper presented at the San Antonio Breast Cancer Symposium. 2012.

15. NABON 2012 Dutch Breast Cancer Guideline, Oncoline. http://www.oncoline.nl/breastcancer. 
16. Giuliano AE, Hawes D, Ballman KV, Whitworth PW, Blumencranz PW, Reintgen DS, Morrow M, Leitch AM, Hunt KK, McCall LM, Abati A, Cote R. Association of occult metastases in sentinel lymph nodes and bone marrow with survival among women with early-stage invasive breast cancer. JAMA 2011;306: 385-93.

17. Gobardhan PD, Elias SG, Madsen EV, Bongers V, Ruitenberg HJ, Perre $\mathrm{Cl}$, van Dalen T. Prognostic value of micrometastases in sentinel lymph nodes of patients with breast carcinoma: a cohort study. Ann Oncol 2009;20:41-8.

18. Hansen NM, Grube B, Ye X, Turner RR, Brenner RJ, Sim MS, Giuliano AE. Impact of micrometastases in the sentinel node of patients with invasive breast cancer. J Clin Oncol 2009;27:4679-4684.

19. Weaver DL, Ashikaga T, Krag DN, Skelly JM, Anderson SJ, Harlow SP, Julian TB, Mamounas EP, Wolmark N. Effect of occult metastases on survival in node-negative breast cancer. N Engl J Med 2011;364: 412-21.

20. de Boer M, van Deurzen CH, van Dijck JA, Borm GF, van Diest PJ, Adang EM, Nortier JW, Rutgers EJ, Seynaeve C, Menke-Pluymers MB, Bult P, Tjan-Heijnen VC. Micrometastases or isolated tumor cells and the outcome of breast cancer. N Engl J Med 2009;361:653-63.

21. Colleoni M, Rotmensz N, Peruzzotti G, Maisonneuve P, Mazzarol G, Pruneri G, Luini A, Intra M, Veronesi P, Galimberti V, Torrisi R, Cardillo A, Goldhirsch A, Viale G. Size of breast cancer metastases in axillary lymph nodes: clinical relevance of minimal lymph node involvement. J Clin Oncol 2005;23:1379-89.

22. Tan LK, Giri D, Hummer AJ, Panageas KS, Brogi E, Norton L, Hudis C, Borgen PI, Cody HS, $3^{\text {rd }}$. Occult axillary node metastases in breast cancer are prognostically significant: results in 368 node-negative patients with 20-year follow-up. J Clin Oncol 2008;26:1803-9.

23. Arpino G, Generali D, Sapino A, Lucia del M, Frassoldati A, de Laurentis $M$, Paolo P, Mustacchi G, Cazzaniga M, De Placido S, Conte P, Cappelletti M, Zanoni V, Antonelli A, Martinotti M, Puglisi F, Berruti A, Bottini A, Dogliotti L. Gene expression profiling in breast cancer: a clinical perspective. Breast 2013;22:109-20.

24. Cardoso F, Piccart-Gebhart M, Van't Veer L, Rutgers E, Consortium T. The MINDACT trial: the first prospective clinical validation of a genomic tool. Mol Oncol 2007;1:246-51.

25. Straver ME, Meijnen P, van Tienhoven G, van de Velde CJ, Mansel RE, Bogaerts J, Demonty G, Duez N, Cataliotti L, Klinkenbijl J, Westenberg HA, van der Mijle H, Hurkmans C, Rutgers EJ. Role of axillary clearance after a tumor-positive sentinel node in the administration of adjuvant therapy in early breast cancer. J Clin Oncol 2010;28:731-7.

26. Aigner J, Smetanay K, Hof H, Sinn HP, Sohn C, Schneeweiss A, Marme F. Omission of axillary dissection according to ACOSOG Z0011: impact on adjuvant treatment recommendations. Ann Surg Oncol 2013; 20:1538-44.

27. Meretoja TJ, Audisio RA, Heikkila PS, Bori R, Sejben I, Regitnig P, Luschin-Ebengreuth G, Zgajnar J, Perhavec A, Gazic B, Lazar G, Takacs T, Kovari B, Saidan ZA, Nadeem RM, Castellano I, Sapino A, Bianchi S, Vezzosi V, Barranger E, Lousquy R, Arisio R, Foschini MP, Imoto S, Kamma H, Tvedskov TF, Jensen MB, Cserni G, Leidenius $\mathrm{MH}$. International multicenter tool to predict the risk of four or more tumor-positive axillary lymph nodes in breast cancer patients with sentinel node macrometastases. Breast Cancer Res Treat 2013;138:817-27.

28. Early Breast Cancer Trialists' Collaborative G. Effects of chemotherapy and hormonal therapy for early breast cancer on recurrence and 15-year survival: an overview of the randomised trials. Lancet 2005;365:1687-717.

29. Early Breast Cancer Trialists' Collaborative G, Darby S, McGale P, Correa C, Taylor C, Arriagada R, Clarke M, Cutter D, Davies C, Ewertz M, Godwin J, Gray R, Pierce L, Whelan T, Wang Y, Peto R. Effect of radiotherapy after breast-conserving surgery on 10-year recurrence and 15-year breast cancer death: meta-analysis of individual patient data for 10,801 women in 17 randomised trials. Lancet 2011; 378:1707-16.

30. Heuts EM, van der Ent FW, von Meyenfeldt MF, Voogd AC. Internal mammary lymph drainage and sentinel node biopsy in breast cancer - A study on 1008 patients. Eur J Surg Oncol 2009;35:252-7.

31. Poortmans P. Irradiation of the internal mammary and medial spraclavicular lymph nodes in stage I to III breast cancer: 10 years results of the EORTC Radiation Oncology and Breast Cancer Groups phase III trial 22922/10925. Paper presented at the 2013 European Cancer Congress (ECC2013), Amsterdam. 2013. 


\section{Chapter}

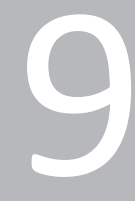

Three-dimensional breast radiotherapy and the elective radiation dose at the sentinel lymph node site in breast cancer

L.M. van Roozendaal

RJ. Schipper

L.H.M. Smit

B.T. Brans

R.G.H. Beets-Tan

M.B.I. Lobbes

L.J. Boersma

M.L. Smidt

Ann Surg Oncol. 2015 Feb 24. (Epub ahead of print) 


\section{Abstract}

\section{Background}

Several trials are presently randomizing clinically node negative breast cancer patients treated with breast conserving therapy to sentinel lymph node biopsy (SLNB) or watchful waiting. We aimed to investigate the elective radiation dose at the sentinel lymph node (SLN) site while evaluating two techniques for SLN localization, in breast cancer patients treated with lumpectomy and three-dimensional whole breast radiotherapy.

\section{Methods}

The SLN site of consecutive Tis-2NO breast cancer patients undergoing lumpectomy and forward intensity modulated whole breast radiotherapy was determined by the location of the hotspot on preoperative SPECT/CT and by a surgical clip placed at the removed SLN(s) during SLNB. The radiation dose at the SLN site was subsequently determined on the postoperative radiotherapy planning CT. An elective radiation dose to the SLN site was defined as at least $95 \%$ of the breast dose.

\section{Results}

Of the 42 included patients, the mean percentage of the breast dose on the SLN site was $90 \%$ (SD 26 , range 7-132, median 99), with a non-significant difference between the two techniques (surgical clip or SPECT/CT) $(P=0.608)$. In $32 / 42$ patients (76\%) the SLN site received an elective radiation dose.

\section{Conclusion}

A surgical clip placed at the removed SLN(s) during SLNB proved to be an adequate method of determining the radiation dose at the SLN site when compared with using SPECT/CT. With the use of three-dimensional radiotherapy, the site of the SLN is treated with an elective radiation dose in the majority of patients who are treated with breast conserving therapy. 


\section{Introduction}

The treatment of breast cancer with breast conserving therapy (BCT) as a less invasive alternative to mastectomy was introduced in the early 1980s. The addition of whole breast radiotherapy to lumpectomy resulted in not only lower local recurrence rates but also lower regional recurrence rates ${ }^{1,2}$. A meta-analysis showed that in sentinel lymph node (SLN) negative patients, whole breast radiotherapy after lumpectomy is associated with a lower risk of axillary recurrence (odds ratio $0.29 ; p<0.001)^{3}$. In the ACOSOG Z0011 trial, SLN positive patients treated with BCT were randomized to completion axillary lymph node dissection or watchful waiting ${ }^{4}$. The regional recurrence rate was not increased in the watchful waiting-arm, while it is estimated that $27 \%$ had residual nodal disease that was not surgically removed ${ }^{5}$. It is suggested that these findings might partly be explained by incidental irradiation of the lower axilla by whole breast radiotherapy ${ }^{6,7}$. Studies have shown that up to $73 \%$ of axillary level I and $26 \%$ of level II receive an elective radiation dose with conventional two-dimensional (2D) whole breast radiotherapy ${ }^{8-10}$.

Presently, several independent trials are randomizing clinically node negative breast cancer patients treated with BCT, to sentinel lymph node biopsy (SLNB) or watchful waiting: the Dutch BOOG 2013-08, the Italian SOUND, and the British SNIPE trials ${ }^{11-13}$. Studies have shown that the SLN site is radiated in $78 \%$ to $94 \%$ of the patients using conventional 2D-whole breast radiotherapy ${ }^{14-16}$. Meanwhile, three-dimensional (3D) radiotherapy techniques mostly replaced 2D-radiotherapy. Both 3D-conformal radiotherapy (3D-CRT) and (forward) intensity modulated radiotherapy (IMRT) are considered 3D-radiotherapy techniques. In 3D-CRT, the shape of the radiation field is adapted to the shape of the target volume, but can still lead to high doses in non target volumes. In IMRT, the shape of the high dose volume is as conformal as possible to the 3D shape of the target volume, and furthermore delivers a more homogeneous dose to the target volume than $3 \mathrm{D}-\mathrm{CRT}^{10}$. IMRT can thus be considered as a more advanced and more accurate type of 3D-radiotherapy. It is questionable whether, with a contemporary radiation technique, the SLN still receives an elective radiation dose as more advanced radiotherapy results in more accurate irradiation of the targeted volume, and less dose to non target volumes.

Previous studies on the radiation dose at the SLN site used surgical clips as a marker, representing the postoperative situation of a minimally dissected axilla. As trials are investigating the safety of omitting the SLNB, we were interested in the SLN site in the undissected axilla. For this purpose, preoperative single-photon emission computed tomography (SPECT)/ computer tomography (CT) images seem more appropriate and were therefore performed in a proportion of patients.

We aimed to investigate whether the radiation dose at the surgical clip that is placed at the location of the removed SLN(s) during SLNB, corresponds to the dose at the region of increased tracer uptake (the hotspot) on SPECT/CT, and, furthermore, whether the 
SLN site receives an elective radiation dose, in breast cancer patients treated with lumpectomy, SLNB, and whole breast radiotherapy using forward IMRT.

\section{Methods}

\section{Study population}

Consecutive patients diagnosed with unifocal cTis-2NO breast cancer of carcinoma in situ of the breast between March 2013 and January 2014, and were about to undergo BCT and SLNB in our breast center, were included. Patients receiving completion axillary treatment or with a history of surgery or radiotherapy of the ipsilateral breast or axilla were excluded. The acquisition of informed consent was waived by the medical ethics committee (MEC UM 13-4-019). One group of patients $(n=10)$ had both preoperative SPECT/CT images of the axillary region and a surgical clip placed at the location of the removed SLNs to evaluate if these methods were equivalent, while a second group of patients $(n=32)$ had only the surgical clip placed at the SLN site.

\section{Sentinel lymph node biopsy}

For the SLNB, a total of $80 \mathrm{MBq}$ Technetium-99 nanocolloid (Nanocoll', GE Healthcare, Eindhoven, the Netherlands) was injected intradermally in the periareolar region, followed by lymphoscintigraphic images. Additionally, integrated SPECT/CT images were available in patients that participated in an ongoing prospective study concerning non-invasive nodal staging (MEC UM 12-0-36). All SPECT/CT images were performed using the same positioning and fixation system as used during the radiotherapy planning $\mathrm{CT}$ and treatment, with the patient in supine position, the head in a contoured headrest and both arms above the head in the cranial arm holder combined with two upper arm supports (Posirest ${ }^{\mathrm{TM}}-2$ system, Cablon Medical B.V., Leusden, The Netherlands). Two hours after injection, the SPECT/CT images were obtained (Precedence SPECT/6-slice CT scanner, Philips, Best, The Netherlands).

The $\operatorname{SLN}(\mathrm{s})$ were identified during surgery by using the lymphoscintigraphic images, blue dye (Bleu Patente V; Guerbet, Aulnay-sous-Bois, France) and gamma probe. The surgeon placed surgical clip(s) at the location of the removed $\operatorname{SLN}(\mathrm{s})$.

\section{Radiotherapy planning scans}

All patients were routinely scheduled for a radiotherapy planning CT within five weeks after surgery (SOMATOM Sensation 10, Siemens, Forchheim, Germany). Patients were positioned as described in the previous section. 


\section{Radiation therapy details}

The prescribed breast dose was $16 \times 2.67 \mathrm{~Gy}$ in 23 patients; 16 patients received a modified scheme with a low boost $(21 \times 2.17 \mathrm{~Gy}$ and simultaneous integrated boost (SIB) of $21 \times 2.66 \mathrm{~Gy})$; and three patients with a high boost (23x2.03Gy and SIB of 23x2,66Gy). Delineation of the breast and tumor bed was performed as described earlier ${ }^{17,18}$. In short, for delineation of the breast a radiopaque wire was used to mark the palpable breast tissue. The clinical target volume (CTV) of the breast was defined $5 \mathrm{~mm}$ under the skin surface, in front of the major pectoral muscle and chest wall, borders largely determined by the radiopaque wire. Cranially, the CTV breast did not extend above the superior border of the sternoclavicular joint, and the medial border of the CTV did not extend beyond the lateral edge of the sternum. The tumor bed was delineated using all preoperative available information, like mammography, MRI and physical examination. For the CTV of the tumor bed, a margin was applied around the tumor bed of $1.5 \mathrm{~cm}$ minus the minimally free resection margin. The CTV of the tumor bed did not extend into the chest wall, and remained $5 \mathrm{~mm}$ beneath the skin. CTV-planning target volume (PTV) expansion was $5 \mathrm{~mm}$ in all directions both for the breast and the tumor bed, except towards the skin surface where no CTV-PTV margin was taken.

Forward IMRT technique was used for treatment planning as described earlier ${ }^{18}$. With this technique we always start with two open tangential beams, and we optimize the dose distribution with additional segments and/or beams. In our patient population we found that we need additional segments (<3 segments) in one-third of the patients, and additional beams ( $<2$ beams) in two-thirds of the patients.

The dose-homogeneity constraints for the planning target volumes was kept to vary between $95 \%$ and $107 \%$ of the prescribed dose. The lung constraints consisted of a central lung distance below $3 \mathrm{~cm}$, the mean lung dose had to be $<7.5 \mathrm{~Gy}$, the heart constraints consisted of a Maximum Heart Distance below $1 \mathrm{~cm}$, the heart volume receiving $>10 \mathrm{~Gy}$ had to be $<5 \%$, and the heart volume receiving $>5 \mathrm{~Gy}$ had to be $<10 \%{ }^{19}$. Moderately voluntary breath holding techniques to reduce heart dose in left-sided breast cancer were applied ${ }^{18}$.

The radiation oncologists and radiation technicians were blinded for the study aim and procedure at time of treatment of all patients.

\section{Data collection and statistical analyses}

Data concerning diagnosis and treatment were collected. The $x, y$ and $z$ axis coordinates of the center of the SPECT hotspot were determined on SPECT/CT, after which they were converted by using the $x, y$ and $z$ axis coordinates $(0,0,0)$ of the planning CT scan, followed by visualisation of these coordinates on the planning CT scan. The surgical clip was also visualized on the planning CT scan. The corresponding radiation dose, expressed as a percentage of the prescribed breast dose, was determined at the location of the SPECT hotspot and the surgical clip, at the center of these points in both 
techniques. In case of multiple clips due to the removal of several SLNs, the lowest radiation dose was obtained. An elective radiation dose to the SLN site was defined as at least $95 \%$ of the prescribed breast dose.

Descriptive categorical data are presented as proportions and absolute numbers. Continuous variables are presented as means with standard deviations (SD). The BlandAltman and one sample T-test was used to analyze the agreement between the radiation dose delivered to the surgical clip and the hotspot, with $95 \%$ limits of agreement. Fisher's exact test was used to analyze if the location of the tumor in the breast, the size of the breast, or a left versus right-sided lesion were predictors for not receiving an elective radiation dose at the SLN site, and binary logistic regression for body mass index, radiation therapy boost, and a tumor located in the laterocranial quadrant. Statistical analyses were performed using Statistical Package for the Social Sciences (IBM SPSS, version 20.0, Chicago, Illinois, USA). A P-value of $<0.05$ was considered statistically significant.

\section{Results}

Mean age of the ten patients with both SPECT/CT images and a surgical clip was 63 years (SD 11, range 50-79, median 63) and mean tumor size was $12 \mathrm{~mm}$ (SD 8, range 4-26, median 11). Radiation dose on the SPECT hotspot and surgical clip of the ten patients are presented in Table 9.1. Mean radiation dose on SPECT hotspot was 38.92 Gy (SD 8, range 20-45) and on surgical clip 38.62 Gy (SD 9, range 16-45). The mean percentage of the breast dose at the SPECT hotspot and the surgical clip was $89 \%$ (SD 17, range 48-105) and $88 \%$ (SD 20, range 37-104) respectively. The mean difference between the two methods was -0.0076 (95\% limits of agreement \pm 0.0899$)(P=0.608)$.

Table 9.1 Radiation dose and percentage of prescribed whole breast dose on SPECT hotspot and surgical clip in ten patients.

\begin{tabular}{|c|c|c|c|c|c|c|}
\hline & \multirow[b]{2}{*}{$\begin{array}{l}\text { Radiation } \\
\text { scheme to the } \\
\text { whole breast }\end{array}$} & \multirow[b]{2}{*}{$\begin{array}{c}\text { Total prescribed } \\
\text { dose to breast (Gy) }\end{array}$} & \multicolumn{2}{|r|}{ SPECT hotspot } & \multicolumn{2}{|r|}{ Surgical clip } \\
\hline & & & $\begin{array}{l}\text { Dose } \\
\text { (Gy) }\end{array}$ & $\begin{array}{l}\text { Percentage of } \\
\text { total breast dose (\%) }\end{array}$ & $\begin{array}{l}\text { Dose } \\
\text { (Gy) }\end{array}$ & $\begin{array}{c}\text { Percentage of } \\
\text { total breast dose (\%) }\end{array}$ \\
\hline 1 & $16 \times 2.67$ & 42.72 & 35.50 & 83.10 & 37.20 & 87.08 \\
\hline 2 & $16 \times 2.67$ & 42.72 & 44.60 & 104.40 & 44.58 & 104.35 \\
\hline 3 & $16 \times 2.67$ & 42.72 & 32.40 & 75.84 & 30.88 & 72.28 \\
\hline 4 & $16 \times 2.67$ & 42.72 & 41.50 & 97.14 & 41.10 & 96.21 \\
\hline 5 & $16 \times 2.67$ & 42.72 & 20.40 & 47.75 & 15.90 & 37.22 \\
\hline 6 & $16 \times 2.67$ & 42.72 & 41.50 & 97.14 & 41.65 & 97.50 \\
\hline 7 & $21 \times 2.17$ & 45.57 & 42.00 & 92.17 & 43.40 & 95.24 \\
\hline 8 & $21 \times 2.17$ & 45.57 & 42.80 & 93.92 & 43.46 & 95.37 \\
\hline 9 & $21 \times 2.17$ & 45.57 & 43.50 & 95.46 & 45.10 & 98.97 \\
\hline 10 & $16 \times 2.67$ & 42.72 & 44.97 & 105.27 & 42.90 & 100.42 \\
\hline
\end{tabular}

Radiation scheme: the number of $f x$ times dose per $f x$ in Gy to the whole breast. 
Because the percentages of the elective radiation dose at the surgical clip and the SPECT hotspot were in line, further analyses were performed on all 42 patients. Patient demographics and tumor characteristics of the 42 patients are shown in Table 9.2. The percentages of the breast dose on the surgical clip of all patients are shown in Figure 9.1. The mean percentage of the breast dose on the surgical clip was $90 \%$ (SD 26, range 7-132, median 99\%). The SLN site received at least an elective radiation dose in 32 patients (76\%). An example of a patient with an elective radiation dose on the surgical clip is shown in Figure 9.2. In 35 of 42 patients (83\%) at least $90 \%$ of the breast dose was delivered, in $36(86 \%)$ at least $80 \%$, and in 37 (88\%) at least 50\% (Table 9.3). The location of the tumor in the breast, the size of the breast, a left versus right-sided lesion or body mass index, and the combination of radiation therapy boost with a tumor located in the laterocranial quadrant, did not influence the elective radiation dose at the SLN site.

Table 9.2 Patient demographics and tumor characteristics of all patients.

\begin{tabular}{|c|c|}
\hline Characteristic & Value \\
\hline Number of patients & 42 \\
\hline \multicolumn{2}{|l|}{ Age, years } \\
\hline Mean (SD) & 66 (9) \\
\hline Range & $48-81$ \\
\hline \multicolumn{2}{|l|}{ Tumor size, mm } \\
\hline Mean (SD) & $17(10)$ \\
\hline Range & $4-50$ \\
\hline \multicolumn{2}{|l|}{ Number of sentinel lymph nodes removed } \\
\hline Mean (SD) & $1.45(0.8)$ \\
\hline Range & $1-4$ \\
\hline \multicolumn{2}{|c|}{ Number of non-sentinel lymph nodes removed } \\
\hline Mean $(S D)$ & $0.5(0.8)$ \\
\hline Range & $0-3$ \\
\hline \multicolumn{2}{|l|}{ pT-stadium, N (\%) } \\
\hline Tis & $3(7)$ \\
\hline $\mathrm{T} 1$ & $29(69)$ \\
\hline $\mathrm{T} 2$ & $10(24)$ \\
\hline \multicolumn{2}{|l|}{ pN-stadium, N (\%) } \\
\hline pNO & $36(86)$ \\
\hline $\mathrm{pNO}(\mathrm{i}+)$ & $1(2)$ \\
\hline pN1mi & $4(10)$ \\
\hline $\mathrm{pN} 1$ & $1(2)$ \\
\hline \multicolumn{2}{|l|}{ Tumor type, N (\%) } \\
\hline Ductal carcinoma in situ & $3(7)$ \\
\hline Invasive ductal carcinoma, NOS & $32(76)$ \\
\hline Invasive lobular carcinoma, NOS & $3(7)$ \\
\hline Other & $4(10)$ \\
\hline \multicolumn{2}{|l|}{ Affected side, N (\%) } \\
\hline Left & $21(50)$ \\
\hline Right & $21(50)$ \\
\hline
\end{tabular}


Table 9.2 (continued).

$\begin{array}{lc}\text { Characteristic } & \text { Value } \\ \text { Breast cup size, N (\%) } & 2(5) \\ \text { A } & 12(29) \\ \text { B } & 13(31) \\ \text { C } & 5(12) \\ \text { D } & 3(7) \\ \text { E } & 2(5) \\ \text { F } & 2(5) \\ \text { G } & 3(7) \\ \text { Unknown } & \\ \text { Location of tumor in breast, N (\%) } & 23(55) \\ \text { Laterocranial quadrant } & 7(17) \\ \text { Laterocaudal quadrant } & 5(12) \\ \text { Mediocranial quadrant } & 7(17) \\ \text { Mediocaudal quadrant } & \\ \text { BMI score, } n \text { (\%) } & 17(41) \\ \text { BMI 18.5-25 } & 17(41) \\ \text { BMI 25-30 } & 6(14) \\ \text { BMI 30-35 } & 2(5) \\ \text { BMI >35 } & \end{array}$

$\mathrm{N}$ : number of cases. SD: standard deviation. NOS: not otherwise specified. BMI: Body mass index.

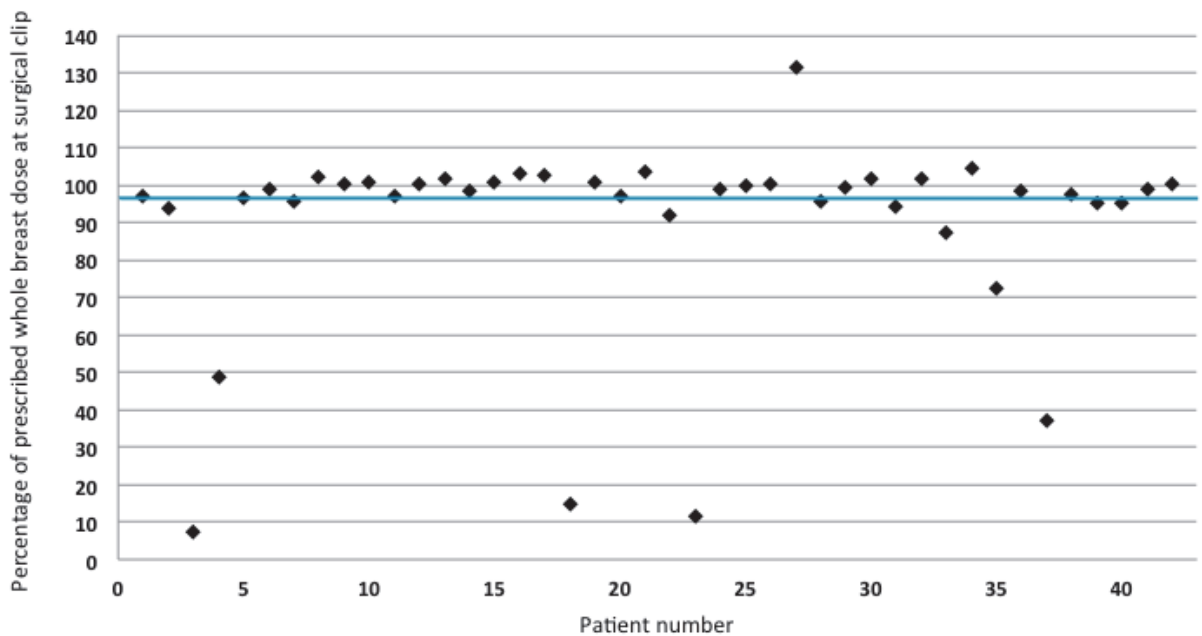

Figure 9.1 Percentage of prescribed whole breast dose on the surgical clip at the sentinel lymph node site in 42 patients. The blue line represents $95 \%$ of the prescribed whole breast dose at the surgical clip. 

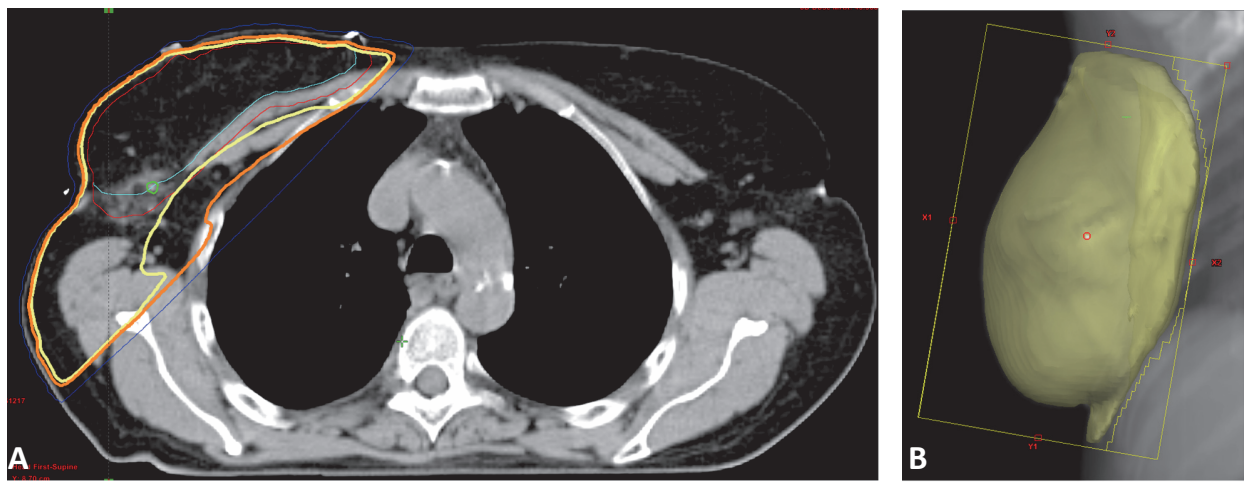

Figure 9.2 Figure 9.2A represents the radiotherapy planning CT of a study patient after lumpectomy and sentinel lymph node biopsy, who was found to have an elective radiation dose at the surgical clip. The breast tumor of this patient was located in the mediocaudal quadrant of the breast; the tumorbed is not visible on this CT image. The yellow line contouring the breast represents the $95 \%$ isodose, i.e. all spots on this line received at least $95 \%$ of the prescribed dose. The orange line represents the $90 \%$ isodose, and the dark blue line the $50 \%$ isodose. The light blue line represents the clinical target volume of the breast and the red line the planning target volume of the breast. The surgical clip at the SLN site is contoured by a green line. In Figure 9.2B the Beam's Eye View of the same patient is shown with the transparent yellow area representing the $95 \%$ isodose coverage and the green spot representing the surgical clip at the SLN site.

Table 9.3 Number of patients receiving at least $95,90,85,80$ or $50 \%$ of the prescribed whole breast dose on surgical clip.

$\begin{array}{lcc}\text { Percentage of prescribed whole breast dose } & \text { Number of patients } & \text { Percentage of patients } \\ \geq 95 \% & 32 & 76 \\ \geq 90 \% & 35 & 83 \\ \geq 85 \% & 36 & 86 \\ \geq 80 \% & 36 & 86 \\ \geq 50 \% & 37 & 88\end{array}$

\section{Discussion}

This study aimed to investigate the incidental radiation dose to the SLN site in breast cancer patients treated with lumpectomy followed by whole breast radiotherapy using forward IMRT, to illustrate incidental treatment of the SLN in trials investigating the safety of omitting the SLNB. A surgical clip placed at the location of the removed SLN(s) during SLNB proved to be an adequate method to determine the radiation dose at the SLN site, when compared to using SPECT/CT images. An elective radiation dose, i.e. $>95 \%$ of the total breast dose, was delivered to the SLN site in $76 \%$ of the patients. 
The hotspot on SPECT/CT represents the preoperative localization of the SLN site in an undissected axilla. This method therefore seems appropriate to simulate the situation where the SLNB is omitted. The SLN site can be visualized without postoperative effects, such as hematoma or seroma, which can influence the configuration of the lymph nodes within the axilla. However, there are some limitations in the use of SPECT/CT for accurate localization of the SLN site. At first, the attenuation of the SPECT and CT images can be technically challenging, for example due to respiratory motion artifacts $^{20}$. Second, the composition of the axilla could change by (oncoplastic) surgery of the breast. In that case, the surgical clip method might be more appropriate for adequate SLN localization. Results of our study showed however, that the percentage of the prescribed breast dose at the hotspot was in line with the surgical clip in ten patients.

3D-CRT and forward IMRT has now mostly replaced conventional 2D-radiotherapy for breast irradiation. The benefit of 3D-radiotherapy includes more accurate irradiation of the targeted volume, and a reduced toxicity to surrounding tissue. In whole breast radiotherapy this might result in a decreased incidental radiation dose to, among others, the axilla, with the hypothetical advantage of a decreased risk of lymphedema and fibrosis ${ }^{21}$. On the contrary, a reduced dose to the axilla could negatively affect the incidental benefit of a decreased regional recurrence rate in patients treated with $\mathrm{BCT}^{3}$. As conventional 2D-radiotherapy instead of 3D-radiotherapy techniques were standardly used in the ACOSOG Z0011 trial, it is suggested that radiotherapy should be extended to include axillary level I-II to maintain excellent regional control when applying Z0011-results in today's practice ${ }^{22}$. Indeed, Kataria et al. showed a relatively small, although significant, difference in the axillary radiation dose when comparing conventional 2D-radiotherapy to 3D-CRT or IMRT. The mean percentage of axillary level I that received at least $90 \%$ of the total breast dose was $73 \%$ for conventional 2Dradiotherapy, and $57 \%(p=0.029)$ for 3D-CRT, and $49 \%(p=0.029)$ for IMRT, and of level II the mean percentage was $25 \%, 41 \%(p=0.028)$, and $35 \%(p=0.068)$, respectively ${ }^{10}$. The current study was the first to investigate the radiation dose to the SLN site with 3Dbreast radiotherapy, more specifically, with IMRT. The elective radiation dose to the SLN in $76 \%$ of the patients in our study is in line with the reported percentage of $78 \%$ of Rabinovitch et al. and lower than the $85 \%$ of Schlembach et al. and the $94 \%$ of Chung et al., which were all studies using conventional 2D-breast radiotherapy ${ }^{14-16}$. As described earlier, IMRT can be considered as a more advanced and more accurate type of 3Dradiotherapy, when compared to 3D-CRT. Together with results of the study of Kataria et al. $^{10}$, it is likely that a higher percentage of patients will have an elective radiation dose to the SLN when evaluating 3D-CRT. Therefore, we find that even with contemporarary 3D-techniques, the SLN still receives an adequate dose in the majority of patients. 
The location of the tumor in the breast, the size of the breast, a left versus right-sided lesion, a radiation therapy boost, and body mass index are sometimes referred to as factors influencing the amount of radiation to the axilla. Our study failed to demonstrate a relation between these factors and not receiving an elective radiation dose at the SLN site. The location of the SLN posterior or high in the axilla is more likely to explain why these patients have a lower radiation dose at the SLN, but we did not analyze this in our cohort. A study of Uren et al. showed that the axillary SLN is located anterior or central in axillary level $I$ in $83 \%$, and the remaining proportion more posterior in level $\mathrm{I}$, or in level $\mathrm{II}_{-1 \mathrm{I}^{23}}$. The site of the tracer injection for SLNB furthermore influences the drainage pattern and thus the location where the SLN appears. In periareolar injections, as performed in our cohort, a higher proportion of patients have drainage to the anterior region of axillary level I $(57 \%)$, compared to peritumoral injections (46\%) $(P=0.042)^{23}$.

In the current study, an elective radiation dose was delivered to the SLN site in $76 \%$ of the patients, and in $86 \%$ the SLN site received at least $85 \%$ of the elective radiation dose (Table 9.3). The optimal dose for curative radiation of metastatic disease in lymph nodes is questionable and might be less than $95 \%$ of the prescribed breast dose. Lower doses could result in a decreased effect, but might still be effective in subclinical disease. Evidence exists for sufficient control of several cancer types with low radiation doses ranging from 10 to $30 \mathrm{~Gy}^{24,25}$. Withers et al. proposed that the dose response curve for microscopic disease is different from the curve for macroscopic disease, and that a lower radiation dose in these patients is probably equally effective ${ }^{26}$. Because the amount of residual subclinical disease differs among patients, it is however not possible to predefine the elective radiation dose for the individual patient.

For the comparison of SPECT/CT and the surgical clip as methods for determining the elective radiation dose at the SLN site, data of ten patients was used in our study. It is not expected that results would be affected when more patients would have been analyzed, because the percentage of patients receiving an elective radiation dose at the SLN site is comparable to what is shown in available literature ${ }^{14-16}$. Final analyses in this study were performed on existing radiation therapy plans of 42 patients, and we did not assess delineation and inter-observer variation, but the radiation oncologists and radiation technicians were blinded for the study aim and procedure at time of treatment of all patients. Despite the growing popularity of partial breast radiotherapy, we did not include patients receiving this treatment to our analysis, because in the Netherlands it is almost only applied within clinical trials and because the mentioned trials investigating the safety of omitting the SLNB only include clinically node negative breast cancer patients when they are treated with lumpectomy and whole breast radiotherapy ${ }^{11-13}$. It can be expected that for partial breast radiotherapy, the amount of 
radiation to the SLN is highly influenced by the location of the tumor in the breast, while this was not the case in our cohort.

In conclusion, a surgical clip placed at the location of the removed SLN during SLNB proved to be an adequate method of determining the radiation dose at the SLN site when compared with using SPECT/CT images. With the use of 3D-radiotherapy, the site of the SLN is treated with an elective radiation dose in the majority of patients who are treated with BCT. Whole breast radiotherapy after lumpectomy in patients in whom the SLNB is omitted might therefore help to prevent an increase of the regional recurrence rate. The safety of omitting the SLNB should be confirmed by the initiated randomized controlled trials. 


\section{References}

1. Fisher B, Anderson S, Bryant J, Margolese RG, Deutsch M, Fisher ER, Jeong JH, Wolmark N. Twenty-year follow-up of a randomized trial comparing total mastectomy, lumpectomy, and lumpectomy plus irradiation for the treatment of invasive breast cancer. N Engl J Med 2002;347:1233-41.

2. Early Breast Cancer Trialists Collaborative Group (EBCTCG), Darby S, McGale P, Correa C, Taylor C, Arriagada R, Clarke M, Cutter D, Davies C, Ewertz M, Godwin J, Gray R, Pierce L, Whelan T, Wang Y, Peto $\mathrm{R}$. Effect of radiotherapy after breast-conserving surgery on 10-year recurrence and 15-year breast cancer death: meta-analysis of individual patient data for 10,801 women in 17 randomised trials. Lancet 2011;378:1707-16.

3. van Wely BJ, Teerenstra S, Schinagl DA, Aufenacker TJ, de Wilt JH, Strobbe L. Systematic review of the effect of external beam radiation therapy to the breast on axillary recurrence after negative sentinel lymph node biopsy. Br J Surg 2011;98:326-33.

4. Giuliano AE, Hunt KK, Ballman KV, Beitsch PD, Whitworth PW, Blumencranz PW, Leitch AM, Saha S, McCall LM, Morrow M. Axillary dissection vs no axillary dissection in women with invasive breast cancer and sentinel node metastasis: a randomized clinical trial. JAMA 2011;305:569-75.

5. Giuliano AE, McCall L, Beitsch P, Whitworth PW, Blumencranz P, Leitch AM, Saha S, Hunt KK, Morrow M, Ballman K. Locoregional recurrence after sentinel lymph node dissection with or without axillary dissection in patients with sentinel lymph node metastases: the American College of Surgeons Oncology Group Z0011 randomized trial. Ann Surg 2010;252:426-32; discussion 432-3.

6. Alco G, Dincer M. Are the standard tangential breast irradiation fields used in the ACOSOG Z0011 trial really covering the entire axilla? Ann Surg 2013;257:e1.

7. Belkacemi Y, Allab-Pan Q, Bigorie V, Khodari W, Beaussart P, Totobenazara JL, Mege JP, Caillet P, Pigneur F, Dao TH, Salmon R, Calitchi E, Bosc R. The standard tangential fields used for breast irradiation do not allow optimal coverage and dose distribution in axillary levels I-II and the sentinel node area. Ann Oncol 2013;24:2023-8.

8. Reznik J, Cicchetti MG, Degaspe B, Fitzgerald TJ. Analysis of axillary coverage during tangential radiation therapy to the breast. Int J Radiat Oncol Biol Phys 2005;61:163-8.

9. Reed DR, Lindsley SK, Mann GN, Austin-Seymour M, Korssjoen T, Anderson BO, Moe R. Axillary lymph node dose with tangential breast irradiation. Int J Radiat Oncol Biol Phys 2005;61:358-64.

10. Kataria T, Bisht SS, Gupta D, Goyal S, Jassal K, Abhishek A, Sharma K, Pareek P, Kumar V, Jain S, Tayal M, Karthikeyan $\mathrm{N}$. Incidental radiation to axilla in early breast cancer treated with intensity modulated tangents and comparison with conventional and 3D conformal tangents. Breast 2013;22:1125-9.

11. van Roozendaal LM, de Wilt JHW, Smidt ML. Clinically node negative breast cancer patients undergoing breast conserving therapy: follow-up versus sentinel lymph node biopsy, in San Antonio Breast Cancer Symposium. 2012, Cancer Research 72(24):567s.

12. Gentilini O, Veronesi U. Abandoning sentinel lymph node biopsy in early breast cancer? A new trial in progress at the European Institute of Oncology of Milan (SOUND: Sentinel node vs Observation after axillary UltraSouND). Breast 2012;21:678-81.

13. Nadeem RM. The feasibility of SNIPE trial; sentinel lymph node biopsy vs. no-SLNB In patients with early breast cancer. Cancer Res 2013;73(24 Suppl).

14. Rabinovitch R, Ballonoff A, Newman F, Finlayson C. Evaluation of breast sentinel lymph node coverage by standard radiation therapy fields. Int J Radiat Oncol Biol Phys 2008;70:1468-71.

15. Schlembach PJ, Buchholz TA, Ross MI, Kirsner SM, Salas GJ, Strom EA, McNeese MD, Perkins GH, Hunt KK. Relationship of sentinel and axillary level I-II lymph nodes to tangential fields used in breast irradiation. Int J Radiat Oncol Biol Phys 2001;51:671-8.

16. Chung MA, DiPetrillo T, Hernandez S, Masko G, Wazer D, Cady B. Treatment of the axilla by tangential breast radiotherapy in women with invasive breast cancer. Am J Surg 2002;184:401-2.

17. Boersma LJ, Janssen T, Elkhuizen PH, Poortmans P, van der Sangen M, Scholten AN, Hanbeukers B, Duppen JC, Hurkmans C, van Vliet C. Reducing interobserver variation of boost-CTV delineation in breast conserving radiation therapy using a pre-operative CT and delineation guidelines. Radiother Oncol 2012; 103:178-82. 
18. Peulen H, Hanbeukers B, Boersma L, van Baardwijk A, van den Ende P, Houben R, Jager J, Murrer L, Borger J. Forward intensity-modulated radiotherapy planning in breast cancer to improve dose homogeneity: feasibility of class solutions. Int J Radiat Oncol Biol Phys 2012;82:394-400.

19. Hurkmans CW, Borger JH, Bos LJ, van der Horst A, Pieters BR, Lebesque JV, Mijnheer BJ. Cardiac and lung complication probabilities after breast cancer irradiation. Radiother Oncol 2000;55:145-51.

20. Seo Y, Mari C, Hasegawa BH. Technological development and advances in single-photon emission computed tomography/computed tomography. Semin Nucl Med 2008;38:177-98.

21. Bentzen SM, Dische S. Morbidity related to axillary irradiation in the treatment of breast cancer. Acta Oncol 2000;39:337-47.

22. Haffty BG, Hunt KK, Harris JR, Buchholz TA. Positive sentinel nodes without axillary dissection: implications for the radiation oncologist. J Clin Oncol 2011;29:4479-81.

23. Uren RF, Howman-GilesR, Chung DK, Spillane AJ, Noushi F, Gillett D, Gluch L, Mak C, West R, Briody J, Carmalt H. SPECT/CT scans allow precise anatomical location of sentinel lymph nodes in breast cancer and redefine lymphatic drainage from the breast to the axilla. Breast 2012;21:480-6.

24. Hatfield P, Cooper R, Sebag-Montefiore D. Involved-field, low-dose chemoradiotherapy for early-stage anal carcinoma. Int J Radiat Oncol Biol Phys 2008;70:419-24.

25. Marks LB. A standard dose of radiation for "microscopic disease" is not appropriate. Cancer 1990; 66: 2498-502.

26. Withers HR, Suwinski R. Radiation dose response for subclinical metastases. Semin Radiat Oncol 1998; 8:224-8. 


\section{Chapter 10}

Discussion and recommendations 
Chapter 10 


\section{Discussion and recommendations}

The goal of this thesis was to explore new methods for a tailored made axillary treatment plan for patients with breast, in order to minimize morbidity without compromising overall survival of the breast cancer survivors. This thesis presents the results of the validation of current available nodal (re-)staging techniques (axillary ultrasound and standard T2-weigthed MRI) and of modern MRI techniques (diffusion weighted MRI and lymph node specific contrast-enhanced axillary MRI). In addition, the simulated potential impact on patient treatment when implementing such a new nodal staging strategy is shown.

\section{Primary nodal staging}

In case of a sentinel lymph node metastasis, a complementary axillary lymph node dissection (ALND) was always indicated in the past. Since this paradigm is overthrown in many patients, the additional benefit of the standard performance of an axillary ultrasound in each patient with breast cancer is questionable ${ }^{1}$.

In chapter 2 we have shown that a negative axillary ultrasound can exclude advanced (pN2-3) nodal disease in virtually all patients of a general breast cancer population with a negative predictive value (NPV) of $95.5 \%$. Nevertheless an axillary ultrasound cannot accurately differentiate between pN1 and pN2-3 disease (NPV of 58.8\%). More important, if axillary ultrasound suggest pN0, still $16 \%$ of the patients will have macrometastatic axillary lymph nodes, and is thus not reliable in selecting the true pNO patients. Therefore, it cannot select the patients in whom a sentinel lymph node biopsy procedure can be omitted ${ }^{2}$.

The standard use of axillary ultrasound in every breast cancer patient could induce overtreatment. An analysis on the performance of axillary ultrasound in the patient group eligible for treatment according to the ACOSOG-Z0011 trial design was performed. In this study, axillary ultrasound was not performed routinely ${ }^{3}$. The use of axillary ultrasound would result in a decrease of the number of clinically node negative patients and subsequently an increase in the number of clinically node positive patients, thereby directing patients to an ALND instead of sentinel lymph node biopsy (SLNB). Our study showed that at least six of the 12 patients with a cN1 status would not have been considered for a more axilla-conserving treatment, while their ALND specimen contained only 1-2 macrometastases ${ }^{2}$.

Nonetheless, if clinicians are reluctant to omit further axillary treatment in selected patients with limited sentinel lymph node metastasis, axillary ultrasound may help in more confidently select the eligible patients. Instead of routinely performing axillary ultrasound in all patients with breast cancer, multidisciplinary tumor boards should consider the use of axillary ultrasound on individual base. 


\section{Nodal staging after neoadjuvant systemic therapy}

Patients with breast cancer are increasingly treated with neoadjuvant systemic therapy ${ }^{4}$. One of the objectives of this approach is downsizing of the primary tumor, enabling breast conservation therapy where mastectomy might be indicated initially, ${ }^{5,6}$. Thus, treatment of the breast is tailored to tumor response to neoadjuvant systemic therapy. In contrast, treatment of axillary lymph nodes is mostly based on lymph node status at initial diagnosis. If an axillary lymph node metastasis is confirmed by histopathology before starting neoadjuvant systemic therapy, an ALND is usually indicated, regardless of the response to neoadjuvant systemic therapy. Examination of these ALND specimens showed that after neoadjuvant systemic therapy pathological complete response $(p C R)$ of axillary lymph node metastases is achieved in $20-42 \%{ }^{7-10}$.

The challenge lies in how to identify these ypNO patients. As a less invasive technique (compared to ALND), performance of SLNB after neoadjuvant systemic therapy was investigated to identify patients with axillary pCR. Although this approach seems appropriate in clinically node negative patients, it is not accurate enough in clinically node positive patients ${ }^{11-14}$. First, the identification rate of the sentinel lymph node decreases to $81-94 \%$. Further, the SLNB is false negative in a significant number of patients $(10-20 \%)^{14}$. The false negative rate depends on the number of lymph nodes removed during the SLNB and identification technique. For example, in the SENTINA trial the false negative rate dropped below $10 \%$ only if more than three nodes were removed $^{13}$. Sentinel lymph node biopsy alone cannot be considered sufficiently reliable to omit ALND in patients with a negative SLN after neoadjuvant systemic treatment.

As alternative to post neoadjuvant systemic therapy SLNB, the MARI-procedure (Marking the Axilla with Radioactive lodine seeds) was proposed. This procedure involves the placing of an iodine seed prior to neoadjuvant treatment in the axillary lymph node metastasis. After completion of neoadjuvant systemic therapy, the axillary lymph node containing the seed will be removed. Pathologic examination will be performed to detect whether or not the primarily metastatic node responded to the neoadjuvant systemic therapy ${ }^{15}$. The MARI-procedure thereby overcomes the problem of the decreased identification rate of the sentinel lymph node and was considered promising $^{16}$. If the goal is to identify patients with axillary PCR in order to be able to defer from an ALND, the test demands a high NPV, which is in the MARI-procedure not higher than $83.3 \%{ }^{17}$.

Concluding, both post neoadjuvant systemic therapy SLNB and the MARI procedures cannot be relied upon for safe omission of ALND or axillary radiotherapy yet.

A noninvasive technique for nodal restaging would be preferable, as it could potentially reduce morbidity by avoiding the need for any invasive procedure in case of pathological complete response ${ }^{18}$. In chapter 5 we systematically reviewed the literature on noninvasive nodal restaging techniques in initially node positive breast cancer patients after neoadjuvant systemic treatment. Studies varied widely regarding 
type of neoadjuvant systemic therapy regimens and definitions of clinical and pathological complete response ${ }^{8,19-21}$. Authors proposed clinical examination, axillary ultrasound, MRI and ${ }^{18}$ F-FDG PET-CT as possible noninvasive diagnostic tools to identify patients with axillary PCR. The reported positive predictive value (PPV) varied between $40-100 \%$, with axillary ultrasound as most promising ${ }^{22}$. In general all studies were single center with relatively small numbers of patients included, and therefore not sufficiently reliable for clinical implementation yet. In chapter 6 , we reported on the performance of a nomogram, based on standard available patient and tumor characteristics, to identify the clinically node positive breast cancer patient who are likely to achieve a complete axillary response to neoadjuvant systemic therapy. The model is reasonably accurate in terms of discriminative ability as reflected in an AUC of $0.77(95 \% \mathrm{Cl}$ 0.71-0.82). However, omitting axillary treatment based solely on the nomogram score is not justified with a PPV of $65 \%$ (at axillary PCR prevalence of $34 \%$ ) for the detection of axillary $\mathrm{pCR}^{21}$.

Thus, in line with the primary nodal staging setting, no noninvasive restaging strategy so far exists that can reliably identify the true node negative patients after neoadjuvant systemic therapy.

\section{Nodal staging using non-enhanced axillary MRI}

We hypothesized that MRI (with its superior contrast resolution) is more accurate in identifying nodal metastases compared to axillary ultrasound, and thereby can identify the true pNO patient. In chapter 3 we proved that T2-weighted axillary MRI shows good diagnostic performance (AUC 0.78 , based on node-by-node validation), with a NPV of $86-89 \%$ and a specificity of $87-95 \%$ on patient-level, for the assessment of axillary metastasis in breast cancer patients ${ }^{23}$. Despite the higher signal-to-noise ratio and potentially higher resolution images of 3.0-Tesla MRI, the diagnostic performance did not improve compared to the current available literature ${ }^{24}$. In line with a previous study in rectal cancer, we proved that the visual perception as well as the quantitative analysis of restricted diffusion on diffusion weighted MRI was not at all helpful in differentiating benign from malignant nodes ${ }^{25}$.

In conclusion, the NPV of unenhanced T2-weighted axillary MRI with or without diffusion weighted imaging for nodal staging in patients with breast cancer does not compete with the NPV of SLNB. As a result, T2-weighted MRI and diffusion weighted MRI cannot yet be considered as a good alternative to the current invasive staging procedures for selecting the true pNO breast cancer patient..

\section{Nodal staging using contrast-enhanced axillary MRI}

A potentially promising method that may improve nodal staging in MRI is the use of a lymph node specific MR contrast agents. Ultrasmall super paramagnetic iron oxide (USPIO)-showed the highest sensitivity and specificity for axillary lymph node 
metastases ${ }^{26}$. However, USPIO is not approved by the Food and Drug Administration. Alternatively, gadofosveset MR blood pool agent was introduced in 2002 to be valuable as a lymph node specific contrast agent ${ }^{27}$. In patients with rectal cancer, gadofosveset enhanced MRI showed a high NPV for nodal metastases detection, as high as with USPIO-enhanced MRI, suggesting that the true node negative patients can be well identified using this contrast ${ }^{28}$.

In chapter 4 we described a feasibility study in 10 patients on gadofosveset-enhanced axillary MRI for nodal staging in breast cancer patients. Benign axillary lymph nodes could be discriminated from malignant lymph nodes with a sensitivity of $86 \%$ and a specificity of $94 \%$. Positive predictive value and NPV were $75 \%$ and $97 \%$, respectively ${ }^{29}$. The diagnostic performance of gadofosveset-enhanced MRI seems to be superior to other low-weighted gadolinium enhanced MRI lymph node studies ${ }^{26}$. In addition the NPV of gadofosveset-enhanced MRI (97\%) exceeds the NPV of 91\% in other gadolinium enhanced MRI studies, suggesting it can identify the true pNO patient with breast cancer

In chapter 7 we investigated the diagnostic performance of a gadofosveset-enhanced axillary MRI for nodal (re-)staging in a second cohort of 73 patients with breast cancer. Two radiologists independently assessed each lymph node, first on standard MRI, subsequently adjusting their assessment on the gadofosveset-enhanced MRI. Overall, node-by-node validation, for reader 1 , standard and gadofosveset-enhanced MRI showed similar diagnostic performance with an AUC of 0.78 and 0.80 respectively; while for reader 2 it was 0.79 and 0.71 . For patient-by-patient validation, the AUC of standard and gadofosveset-enhanced MRI were 0.89 and 0.82 for reader 1 and 0.81 and 0.77 for reader 2 , respectively.

The addition of gadofosveset-enhanced MRI images initially did not show improvement of the radiologist's performances on patient level. While reader 2 was a breast imaging specialized radiologist and not familiar with interpreting gadofosveset-enhanced MRI, reader 1 had the highest experience in rectal cancer nodal staging with gadofosveset MRI but was not a subspecialized breast radiologist and hence less familiar with the breast tumor lymph nodal pathways of spread in the axilla. Both readers had feedback on their errors after the first 50 patients. Reader 1 significantly improved in performance after feedback at 50 patients (from an AUC of 0.75 to 0.94 ), probably because he learnt from own mistakes and got to understand after feedback the pathways of breast tumor spread in the axillary nodes. The breast imaging specialized radiologist without any experience in gadofosveset-enhanced MRI nodal staging showed only a slight and non-significant improvement after feedback at 50 patients. This suggests a longer learning curve for interpreting gadofosveset-enhanced MRI of nodal metastases.

Of special interest is the sensitivity of $83 \%$ and NPV of $94 \%$ of gadofosveset-enhanced MRI, suggesting that the gadofosveset-enhanced MRI is able to identify the true (y)pNO patients-Furthermore, an NPV of $94 \%$ approximates the NPV of $97 \%$ of the generally 
accepted but more invasive SLNB ${ }^{30}$. It also outperforms the diagnostic performance of SLNB post-neoadjuvant systemic therapy or the MARI-procedure in patients with clinically node positive breast cancer ${ }^{11-13,16}$

Concluding, the current studies showed that gadofosveset-enhanced MRI has potential as a noninvasive method for nodal (re-)staging in patients with breast cancer. The use of gadofosveset-enhanced axillary MRI can eliminate overtreatment and minimize the morbidity of any invasive nodal staging procedure in breast cancer.

\section{Simulating impact of omitting SLNB}

When a gadofosveset-enhanced axillary MRI would be used to decide to omit surgical nodal staging, some patients with lymph node metastases will be overlooked, since its NPV is not $100 \%$. This could influence the decision to recommend adjuvant systemic therapy. We showed in chapter 8 that the decision to recommend adjuvant systemic treatment changed due to knowledge of the true pathological lymph node status, in addition to patient and tumor characteristics, is only $1.0 \%-3.6 \%$ in a cohort of patient with favorable tumor characteristics (clinically node negative breast cancer patients with negative axillary ultrasound findings, who were treated with breast conserving therapy) ${ }^{31}$. Most conflicting outcomes regarding recommendation of adjuvant systemic treatment were assessed in patients with micrometastases. Although a micrometastasis in the sentinel lymph node is considered to have an impact on the prognosis of patients with low risk primary breast cancer, the presence of micrometastasis will not elevate the risk in such a way, that the benefit of adjuvant systemic treatment is large enough to justify the standard administration of this treatment ${ }^{32-39}$.

Furthermore, several gene-expression profiles have been developed with the aim to select patients who are at risk for recurrence, compared to the use of patient and tumor characteristics only ${ }^{40}$. Gene expression profiles are performed on primary tumor material. Therefore, lymph node sampling is not needed.

Concluding, the impact of the pathological lymph node status in addition to patient and tumor characteristics on adjuvant systemic treatment recommendations in patients with breast cancer is limited. This finding could support the clinical implementation of a gadofosveset-enhanced axillary MRI for nodal staging in breast cancer.

Besides systemic undertreatment there is a risk for regional undertreatment when lymph node metastases are left behind in gadofosveset-enhanced axillary MRI negative patients. If these patients will not be treated regionally and systemically, there is a theoretically higher risk to develop a recurrence in the axillary region. It is reported that whole breast radiotherapy after lumpectomy can eradicate metastasis in the axillary lymph nodes, because of the incidental dose radiotherapy to the axilla using standard tangent radiotherapy techniques ${ }^{41}$. However, there is only limited data on the dose delivered to the sentinel lymph node location, especially when using modern 3-dimensional instead of 2-dimensional radiotherapy techniques, such as 3-dimensional 
conformal radiotherapy (3D-CRT) and (forward) intensity-modulated radiotherapy (IMRT). In chapter 9 we reported that in $32 / 42$ patients (76\%) the sentinel lymph node site received an elective radiation dose using modern radiotherapy techniques ${ }^{42}$. Studies using conventional 2D-breast radiotherapy, reported an elective radiation dose to the SLN site in $78-94 \%$ of patients ${ }^{43-45}$. Therefore, in case of modern radiotherapy techniques as 3D-CRT or IMRT, whole breast radiotherapy after lumpectomy, in patients in whom axillary surgery is omitted depending on imaging, helps to prevent an increase of the regional recurrence rate.

\section{Future perspectives}

Future research should be conducted to confirm the diagnostic performance of gadofosveset-enhanced MRI on patient level in a larger group of patients with breast cancer, preferably in a multi-center setting. Ultimately, clinical outcome studies are needed to understand whether an NPV of $94 \%$ is oncologically safe to replace the current SLNB and ALND.

If these trials will confirm the value of gadofosveset-enhanced MRI, we need to invest in training of breast imaging radiologists to facilitate broad implementation in clinical practice.

At this moment, it is unclear whether the diagnostic performance of gadofosvesetenhanced axillary MRI is influenced by tumor type and receptor status. We know that the diagnostic performance of breast MRI and ${ }^{18}$ F-FDG PET-CT depends on tumor subtype. Therefore this needs to be a subject of future research.

Regarding ${ }^{18}$ F-FDG PET-CT, technical improvements of the PET detector can facilitate depiction of small foci of metabolic activity ${ }^{46}$. One could design dedicated axillary PET detectors, in line with the development of dedicated breast PET devices, of which the results are promising compared to conventional whole body PET-CT devices ${ }^{47}$.

Furthermore, PET tracers other than ${ }^{18} \mathrm{~F}$-FDG may provide better results ${ }^{48}$. Promising results are reported with the use of 3 '-deoxy-3'-fluorothymide (FLT). FLT was found to correlate well with the degree of cell proliferation ${ }^{49}$ and could therefore may be used as parameter for treatment effectiveness during neoadjuvant systemic therapy ${ }^{50,51}$.

\section{Conclusion and recommendations}

The treatment of breast cancer is rapidly evolving, especially with respect to staging and treatment of the axillary lymph nodes. The different trials investigating the omission of ALND and/or radiotherapy after the detection of limited sentinel lymph node metastases have learnt that the extent of the nodal disease in the sentinel lymph node (i.e. isolated tumor cells, micrometastases or macrometastases) is not an adequate indicator of the extent of axillary treatment. Therefore, imaging studies that can determine the extent of metastatic lymph nodes in the entire axilla at diagnosis are warranted. 
We showed that the routine performance of an axillary ultrasound in every patient with breast cancer seems unjustified, since it can introduce overtreatment. Instead of routinely performing axillary ultrasound in patients with breast cancer, multidisciplinary tumor boards should consider the use of axillary ultrasound on an individual bases. Nonetheless, the performance of axillary ultrasound with tissue sampling is still justified in patients with palpable axillary lymph nodes.

With the enormous progress of systemic treatment options in breast cancer, we need noninvasive imaging studies that can assess the response of metastatic lymph nodes to these treatments. Standard T2-weighted MRI or diffusion weighted MRI is not accurate to identify patients with (y)pNO lymph node status. The use of the lymph node specific MR contrast gadofosveset seems promising for this purpose but multicenter studies are needed to understand whether this technique can be generalized. The slightly lower NPV gadofosveset-enhanced axillary MRI could theoretically increase the risk of regional recurrences, yet this disadvantage could well balance against the treatment related morbidity associated with SLNB and ALND. 


\section{References}

1. Houssami N, Turner RM. Staging the axilla in women with breast cancer: the utility of preoperative ultrasound-guided needle biopsy. Cancer Biol Med 2014;11:69-77.

2. Schipper RJ, van Roozendaal LM, de Vries B, Pijnappel RM, Beets-Tan RG, Lobbes MB, Smidt ML. Axillary ultrasound for preoperative nodal staging in breast cancer patients: is it of added value? Breast 2013; 22:1108-13.

3. Giuliano AE, Hunt KK, Ballman KV, Beitsch PD, Whitworth PW, Blumencranz PW, Leitch AM, Saha S, McCall LM, Morrow M. Axillary dissection vs no axillary dissection in women with invasive breast cancer and sentinel node metastasis: a randomized clinical trial. JAMA 2011;305:569-75.

4. Kaufmann M, von Minckwitz G, Mamounas EP, Cameron D, Carey LA, Cristofanilli M, Denkert C, Eiermann W, Gnant M, Harris JR, Karn T, Liedtke C, Mauri D, Rouzier R, Ruckhaeberle E, Semiglazov V, Symmans WF, Tutt A, Pusztai L. Recommendations from an international consensus conference on the current status and future of neoadjuvant systemic therapy in primary breast cancer. Ann Surg Oncol 2012;19:1508-16.

5. Fisher B, Bryant J, Wolmark N, Mamounas E, Brown A, Fisher ER, Wickerham DL, Begovic M, DeCillis A, Robidoux A, Margolese RG, Cruz AB Jr, Hoehn JL, Lees AW, Dimitrov NV, Bear HD. Effect of preoperative chemotherapy on the outcome of women with operable breast cancer. J Clin Oncol 1998;16:2672-85.

6. van der Hage JA, van de Velde CJ, Julien JP, Tubiana-Hulin M, Vandervelden C, Duchateau L. Preoperative chemotherapy in primary operable breast cancer: results from the European Organization for Research and Treatment of Cancer trial 10902. J Clin Oncol 2001;19:4224-37.

7. Alvarado R, Yi M, Le-Petross H, Gilcrease M, Mittendorf EA, Bedrosian I, Hwang RF, Caudle AS, Babiera GV, Akins JS, Kuerer HM, Hunt KK. The role for sentinel lymph node dissection after neoadjuvant chemotherapy in patients who present with node-positive breast cancer. Ann Surg Oncol 2012;19: 3177-84.

8. Koolen BB, Valdes Olmos RA, Wesseling J, Vogel WV, Vincent AD, Gilhuijs KG, Rodenhuis S, Rutgers EJ, Vrancken Peeters MJ. Early assessment of axillary response with (1)(8)F-FDG PET/CT during neoadjuvant chemotherapy in stage II-III breast cancer: implications for surgical management of the axilla. Ann Surg Oncol 2013;20:2227-35.

9. Rouzier R, Extra JM, Klijanienko J, Falcou MC, Asselain B, Vincent-Salomon A, Vielh P, Bourstyn E. Incidence and prognostic significance of complete axillary downstaging after primary chemotherapy in breast cancer patients with T1 to T3 tumors and cytologically proven axillary metastatic lymph nodes. J Clin Oncol 2002;20:1304-10.

10. Straver ME, Rutgers EJ, Russell NS, Oldenburg HS, Rodenhuis S, Wesseling J, Vincent A, Peeters MT. Towards rational axillary treatment in relation to neoadjuvant therapy in breast cancer. Eur J Cancer 2009;45:2284-92.

11. Boughey JC, Suman VJ, Mittendorf EA, Ahrendt GM, Wilke LG, Taback B, Leitch AM, Kuerer HM, Bowling M, Flippo-Morton TS, Byrd DR, Ollila DW, Julian TB, McLaughlin SA, McCall L, Symmans WF, Le-Petross HT, Haffty BG, Buchholz TA, Nelson H, Hunt KK. Alliance for Clinical Trials in, Sentinel lymph node surgery after neoadjuvant chemotherapy in patients with node-positive breast cancer: the ACOSOG Z1071 (Alliance) clinical trial. JAMA 2013;310:1455-61.

12. Boileau JF, Poirier B, Basik M, Holloway CM, Gaboury L, Sideris L, Meterissian S, Arnaout A, Brackstone M, McCready DR, Karp SE, Trop I, Lisbona A, Wright FC, Younan RJ, ProvencherL, Patocskai E, Omeroglu A, Robidoux A. Sentinel Node Biopsy After Neoadjuvant Chemotherapy in Biopsy-Proven Node-Positive Breast Cancer: The SN FNAC Study. J Clin Oncol 2015;33:258-64. .

13. Kuehn T, Bauerfeind I, Fehm T, Fleige B, Hausschild M, Helms G, Lebeau A, Liedtke C, von Minckwitz G, Nekljudova V, Schmatloch S, Schrenk P, Staebler A, Untch M. Sentinel-lymph-node biopsy in patients with breast cancer before and after neoadjuvant chemotherapy (SENTINA): a prospective, multicentre cohort study. Lancet Oncol 2013;14:609-18.

14. Fontein DB, van de Water W, Mieog JS, Liefers GJ, van de Velde CJ. Timing of the sentinel lymph node biopsy in breast cancer patients receiving neoadjuvant therapy - recommendations for clinical guidance. Eur J Surg Oncol 2013;39:417-24. 
15. Straver ME, Loo CE, Alderliesten T, Rutgers EJ, Vrancken Peeters MT. Marking the axilla with radioactive iodine seeds (MARI procedure) may reduce the need for axillary dissection after neoadjuvant chemotherapy for breast cancer. Br J Surg 2010;97:1226-31.

16. Donker M, Straver ME, Wesseling J, Loo CE, Schot M, Drukker CA, van Tinteren H, Sonke GS, Rutgers EJ, Vrancken Peeters MJ. Marking Axillary Lymph Nodes With Radioactive lodine Seeds for Axillary Staging After Neoadjuvant Systemic Treatment in Breast Cancer Patients: The MARI Procedure. Ann Surg 2014.

17. Vugts G, Schipper RJ, Maaskant-Braat AJ, Smidt ML, Nieuwenhuijzen GA. Axillary Response Monitoring After Neoadjuvant Chemotherapy in Breast Cancer: Can We Avoid the Morbidity of Axillary Treatment? Ann Surg 2014.

18. DiSipio T, Rye S, Newman B, Hayes S. Incidence of unilateral arm lymphoedema after breast cancer: a systematic review and meta-analysis. Lancet Oncol 2013;14:500-15.

19. Arimappamagan A, Kadambari D, Srinivasan K, Krishnan R, Elangovan S, Reddy KS. Complete axillary conversion after neoadjuvant chemotherapy in locally advanced breast cancer: a step towards conserving axilla? Indian J Cancer 2004;41:13-7.

20. Hieken TJ, Boughey JC, Jones KN, Shah SS, Glazebrook KN. Imaging response and residual metastatic axillary lymph node disease after neoadjuvant chemotherapy for primary breast cancer. Ann Surg Oncol 2013;20:3199-204.

21. Schipper RJ, Moossdorff M, Nelemans PJ, Nieuwenhuijzen GA, de Vries B, Strobbe LJ, Roumen RM, van den Berkmortel F, Tjan-Heijnen VC, Beets-Tan RG, Lobbes MB, Smidt ML. A model to predict pathologic complete response of axillary lymph nodes to neoadjuvant chemo(immuno)therapy in patients with clinically node-positive breast cancer. Clin Breast Cancer 2014;14:315-22.

22. Schipper RJ, Moossdorff M, Beets-Tan RG, Smidt ML, Lobbes MB. Noninvasive nodal restaging in clinically node positive breast cancer patients after neoadjuvant systemic therapy: A systematic review. Eur J Radiol 2015;84:41-7..

23. Schipper, R.J., M.L. Paiman, R.G. Beets-Tan, P.J. Nelemans, B. de Vries, E.M. Heuts, K.K. van de Vijver, K.B. Keymeulen, B. Brans, M.L. Smidt, and M.B. Lobbes, Diagnostic Performance of Dedicated Axillary T2- and Diffusion-weighted MR Imaging for Nodal Staging in Breast Cancer. Radiology 2014:141167.

24. Scaranelo AM, Eiada R, Jacks LM, Kulkarni SR, Crystal P. Crystal, Accuracy of unenhanced MR imaging in the detection of axillary lymph node metastasis: study of reproducibility and reliability. Radiology 2012;262:425-34.

25. Heijnen LA, Lambregts DM, Mondal D, Martens MH, Riedl RG, Beets GL, Beets-Tan RG. Diffusionweighted MR imaging in primary rectal cancer staging demonstrates but does not characterise lymph nodes. Eur Radiol 2013;23:3354-60.

26. Cooper KL, Meng Y, Harnan S, Ward SE, Fitzgerald P, Papaioannou D, Wyld L, Ingram C, Wilkinson ID, Lorenz E. Positron emission tomography (PET) and magnetic resonance imaging (MRI) for the assessment of axillary lymph node metastases in early breast cancer: systematic review and economic evaluation. Health Technol Assess 2011;15:1-134.

27. Herborn CU, Lauenstein TC, Vogt FM, Lauffer RB, Debatin JF, Ruehm SG. Interstitial MR lymphography with MS-325: characterization of normal and tumor-invaded lymph nodes in a rabbit model. AJR Am J Roentgenol 2002;179:1567-72.

28. Lambregts DM1, Beets GL, Maas M, Kessels AG, Bakers FC, Cappendijk VC, Engelen SM, Lahaye MJ, de Bruïne AP, Lammering G, Leiner T, Verwoerd JL, Wildberger JE, Beets-Tan RG. Accuracy of gadofosveset-enhanced MRI for nodal staging and restaging in rectal cancer. Ann Surg 2011;253:53945.

29. Schipper RJ, Smidt ML, van Roozendaal LM, Castro CJ, de Vries B, Heuts EM, Keymeulen KB, Wildberger $J E$, Lobbes MB, Beets-Tan RG. Noninvasive nodal staging in patients with breast cancer using gadofosveset-enhanced magnetic resonance imaging: a feasibility study. Invest Radiol 2013;48:134-9.

30. Pesek S, Ashikaga T, Krag LE, Krag D. The false-negative rate of sentinel node biopsy in patients with breast cancer: a meta-analysis. World J Surg 2012;36:2239-51.

31. van Roozendaal LM, Schipper RJ, Van de Vijver KK, Haekens CM, Lobbes MB, Tjan-Heijnen VC, de Boer M, Smidt ML. The impact of the pathological lymph node status on adjuvant systemic treatment recommendations in clinically node negative breast cancer patients. Breast Cancer Res Treat 2014; 143: 469-76. 
32. Maaskant-Braat AJ, van de Poll-Franse LV, Voogd AC, Coebergh JW, Roumen RM, Nolthenius-Puylaert MC, Nieuwenhuijzen GA. Sentinel node micrometastases in breast cancer do not affect prognosis: a population-based study. Breast Cancer Res Treat 2011;127:195-203.

33. Giuliano AE, Hawes D, Ballman KV, Whitworth PW, Blumencranz PW, Reintgen DS, Morrow M, Leitch AM, Hunt KK, McCall LM, Abati A, Cote R. Association of occult metastases in sentinel lymph nodes and bone marrow with survival among women with early-stage invasive breast cancer. JAMA 2011;306:38593.

34. Gobardhan PD, Elias SG, Madsen EV, Bongers V, Ruitenberg HJ, Perre $\mathrm{Cl}$, van Dalen T. Prognostic value of micrometastases in sentinel lymph nodes of patients with breast carcinoma: a cohort study. Ann Oncol 2009;20:41-8.

35. Hansen NM, Grube B, Ye X, Turner RR, Brenner RJ, Sim MS, Giuliano AE. Impact of micrometastases in the sentinel node of patients with invasive breast cancer. J Clin Oncol, 2009. 27(28): p. 4679-84.

36. Weaver DL, Ashikaga T, Krag DN, Skelly JM, Anderson SJ, Harlow SP, Julian TB, Mamounas EP, Wolmark N. Effect of occult metastases on survival in node-negative breast cancer. N Engl J Med 2011;364:41221.

37. de Boer M, van Deurzen CH, van Dijck JA, Borm GF, van Diest PJ, Adang EM, Nortier JW, Rutgers EJ, Seynaeve C, Menke-Pluymers MB, Bult P, Tjan-Heijnen VC. Micrometastases or isolated tumor cells and the outcome of breast cancer. N Engl J Med 2009;361:653-63.

38. Colleoni M, Rotmensz N, Peruzzotti G, Maisonneuve P, Mazzarol G, Pruneri G, Luini A, Intra M, Veronesi P, Galimberti V, Torrisi R, Cardillo A, Goldhirsch A, Viale G. Size of breast cancer metastases in axillary lymph nodes: clinical relevance of minimal lymph node involvement. J Clin Oncol 2005;23:1379-89.

39. Tan LK, Giri D, Hummer AJ, Panageas KS, Brogi E, Norton L, Hudis C, Borgen PI, Cody HS 3rd. Occult axillary node metastases in breast cancer are prognostically significant: results in 368 node-negative patients with 20-year follow-up. J Clin Oncol 2008;26:1803-9.

40. Arpino G, Generali D, Sapino A, Del Matro L, Frassoldati A, de Laurentis M, Paolo P, Mustacchi G, Cazzaniga M, De Placido S, Conte P, Cappelletti M, Zanoni V, Antonelli A, Martinotti M, Puglisi F, Berruti A, Bottini A, Dogliotti L. Gene expression profiling in breast cancer: a clinical perspective. Breast 2013; 22:109-20.

41. van Wely BJ, Teerenstra S, SchinagI DA, Aufenacker TJ, de Wilt JH, Strobbe L. Systematic review of the effect of external beam radiation therapy to the breast on axillary recurrence after negative sentinel lymph node biopsy. Br J Surg 2011;98:326-33.

42. van Roozendaal LM, Schipper RJ, Smit LH, Brans BT, Beets-Tan RG, Lobbes MB, Boersma L, Smidt ML. Three-Dimensional Breast Radiotherapy and the Elective Radiation Dose at the Sentinel Lymph Node Site in Breast Cancer. Ann Surg Oncol, 2015.

43. Rabinovitch R, Ballonoff A, Newman F, Finlayson C. Evaluation of breast sentinel lymph node coverage by standard radiation therapy fields. Int J Radiat Oncol Biol Phys 2008;70:1468-71.

44. Schlembach PJ, Buchholz TA, Ross MI, Kirsner SM, Salas GJ, Strom EA, McNeese MD, Perkins GH, Hunt KK. Relationship of sentinel and axillary level I-II lymph nodes to tangential fields used in breast irradiation. Int J Radiat Oncol Biol Phys 2001;51:671-8.

45. Chung MA, DiPetrillo T, Hernandez S, Masko G, Wazer D, Cady B. Treatment of the axilla by tangential breast radiotherapy in women with invasive breast cancer. Am J Surg 2002;184:401-2.

46. El Fakhri G, Surti S, Trott CM, Scheuermann J, Karp JS. Improvement in lesion detection with wholebody oncologic time-of-flight PET. J Nucl Med 2011;52:347-53.

47. Koolen BB, Vogel WV, Vrancken Peeters MJ, Loo CE, Rutgers EJ, Valdes Olmos RA. Molecular Imaging in Breast Cancer: From Whole-Body PET/CT to Dedicated Breast PET. J Oncol 2012;2012:438647.

48. Linden HM, Dehdashti F. Novel methods and tracers for breast cancer imaging. Semin Nucl Med 2013;43:324-9.

49. Bading JR, Shields AF. Imaging of cell proliferation: status and prospects. J Nucl Med 2008;49 Suppl 2: 64S-80S.

50. Kenny LM, Al-Nahhas A, Aboagye EO. Novel PET biomarkers for breast cancer imaging. Nucl Med Commun 2011;32:333-5.

51. Pio BS, Park CK, Pietras R, Hsueh WA, Satyamurthy N, Pegram MD, Czernin J, Phelps ME, Silverman DH. Usefulness of 3'-[F-18]fluoro-3'-deoxythymidine with positron emission tomography in predicting breast cancer response to therapy. Mol Imaging Biol 2006;8:36-42. 
Summary 


\section{Summary}

This thesis addressed several topics on (re-)staging of axillary lymph nodes and the treatment of these nodes in patients with breast cancer. The overall focus of this research was to create opportunities for a more tailored made axillary treatment plan for each patient with breast cancer.

In Chapter $\mathbf{2}$ we describe the capability of axillary ultrasound to differentiate between patients with no ( $\mathrm{pNO}$ ), limited axillary disease (defined as $\mathrm{pN} 1$ ) and those with advanced axillary disease (defined as pN2 or pN3). This was investigated in a group of 564 patients that were treated with primary surgery. After negative axillary ultrasound findings (cN0), pathology showed pN2-N3 disease in $4.4 \%$ of these cases. Further, pN1 disease was seen in $16 \%$ of the cases. When cN1 (1-3 suspicious nodes) was imaged, pathology showed pN2-N3 disease in $41.2 \%$. Concluding, axillary ultrasound is useful to rule out pN2-3 disease in virtually of patients with breast cancer. Nonetheless, it is unreliable in selecting true pNO patients.

In Chapter 3 the diagnostic performance of non-enhanced, axillary T2-weighted and diffusion weighted MR imaging for nodal staging in a population $(n=50)$ of newly diagnosed breast cancer patients was investigated. With respective node-by-node and patient-by-patient validation, T2-weighted MR imaging had a good diagnostic performance. The addition of diffusion weighted MRI and apparent diffusion coefficient (ADC) measurements were of no added value. The NPV of the axillary T2-weighted MRI is good, however it does not approach the NPV of sentinel lymph node biopsy.

The feasibility study in chapter 4 investigated the use of gadofosveset-enhanced axillary MRI for nodal staging in ten breast cancer patients. The axillary lymph nodes showed peak enhancement between 11 and 21 minutes. With an NPV of $97 \%$ gadofosveset-enhanced axillary MRI seems promising in selecting the true pNO patient with breast cancer.

In Chapter 5 we provided a systematic review on noninvasive nodal restaging techniques of the axillary lymph node in initial node positive breast cancer patients after neoadjuvant systemic treatment. After evaluating 987 abstracts, four studies were included. In these studies, clinical examination, axillary ultrasound, breast MRI and whole body FDG PET-CT and a prediction model were investigated. The included studies were often limited by small sample size and definitions of pathological complete response were variable. At present, there is no robust accurate noninvasive restaging technique able to identify patients with axillary pathologic complete response after neoadjuvant treatment. 
Chapter 6 describes the development and accuracy of a nomogram based on standard available parameters of 291 clinically node positive patients, to identify the clinically node positive breast cancer patient who are likely to achieve an axillary complete response to neoadjuvant systemic therapy. The prediction model showed reasonable accuracy with an area under the ROC curve of 0.77 only. Consequently, it is not reliable enough to support the decision to omit axillary treatment in patients with a high nomogram score.

Chapter 7 reported on the diagnostic performance of a gadofosveset-enhanced axillary MRI for nodal staging and restaging in a second cohort of breast cancer patients $(n=73)$. Overall, standard and gadofosveset-enhanced axillary MRI showed similar diagnostic performance. However, after providing feedback to the readers on the first 50 cases, improvement of the diagnostic performance was observed in the following 23 cases. With an NPV of $94 \%$, gadofosveset-enhanced axillary MRI approximates the NPV of $97 \%$ of the generally accepted but more invasive sentinel lymph node biopsy.

Chapter 8 evaluated the impact of ignorance of the true pathological lymph node status in a patient group $(n=303)$ with favorable tumor characteristics on adjuvant systemic treatment recommendations. While $5 \%$ had micrometastatic and $10 \%$ macrometastatic disease in their axillary lymph nodes, adjuvant systemic treatment changed due to the presence of these metastases in only 1.0-3.6\% of the cases, depending using Adjuvant Online! or the Dutch Guideline. So the impact of the pathological lymph node status on adjuvant systemic treatment recommendations in clinically node negative breast cancer patients is limited.

In Chapter 9 patients treated with lumpectomy, followed by whole breast radiotherapy were evaluated regarding the incidental radiation dose delivered to the location of the sentinel lymph node. A preoperative SPECT-CT and a surgical clip placed at the location of the removed sentinel lymph node were used to locate the sentinel lymph node site on the postoperative radiotherapy planning CT. In 32 out of 42 patients, the sentinel lymph node site received an elective radiation dose in patients treated with breast conserving therapy. Whole breast radiotherapy after lumpectomy in patients in whom the sentinel lymph node biopsy is omitted might therefore help to prevent an increase of the regional recurrence rate. 
Samenvatting 


\section{Samenvatting}

Dit proefschrift heeft verschillende aspecten belicht met het betrekking tot het (her-) stadiëren van de axillaire lymfeklieren en de behandeling van deze klieren in patiënten met borstkanker. Het uiteindelijke doel van al deze onderzoeken was het creëren van een meer individueel op maat gemaakt behandelplan van de axillaire lymfeklieren voor elke patiënt met borstkanker.

In hoofdstuk 2 beschrijven wij de mogelijkheden om met behulp van axillaire echografie onderscheidt te maken tussen patiënten zonder axillaire metastasen (pNO), met beperkte axillaire metastasering (gedefinieerd als pN1) en die met uitgebreide axillaire metastasering (gedefinieerd als pN2-pN3). Dit werd onderzocht in een groep van 564 patiënten die primair werden behandeld met chirurgie. Na een negatieve axillaire echografie (cNO), toonde histopathologisch onderzoek in $4.4 \%$ van de patiënten toch nog pN2-pN3. Verder bleek dat bij 16\% van de patiënten sprake was van pN1. Wanneer met behulp van axillaire echografie cN1 werd voorspeld (1-3 axillaire lymfekliermetastasen), toonde histopathologisch onderzoek pN2-pN3 in 41.2\% van de gevallen. Concluderend, echografie is nuttig in het uitsluiten van pN2-pN3 in een algemene populatie van patiënten met borstkanker. Niettemin is echografie onbetrouwbaar in het selecteren van de patiënten met pNO.

In hoofdstuk 3 wordt de diagnostische waarde van axillaire T2-gewogen en diffusie gewogen (DWI) MRI voor lymfeklierstadiëring onderzocht. Zowel op basis van een klierbij-klier en patiënt-per-patiënt validatie, bleek dat T2-gewogen MRI een goede diagnostische waarde heeft. Het toevoegen van DWI en ADC metingen had geen positief effect op de diagnostische waarde. De negatief voorspellende waarde van T2 gewogen MRI voor axillaire lymfeklierstadiëring is goed, echter het benadert niet de NPV van de schildwachtersklierprocedure.

De pilotstudie in hoofdstuk 4 onderzocht het gebruik van een (gadofosveset) contrastversterkte axillaire MRI voor de stadiëring van axillaire lymfeklieren in 10 patiënten. De axillaire lymfeklieren waren het beste te onderscheiden in de tijdsperiode 11-21 minuten na het toedienen van het contrastmiddel. Met een NPV van $97 \%$ bleek de gadofosveset versterkte MRI veelbelovend in het selecteren van borstkanker patiënten met pNO.

In hoofdstuk $\mathbf{5}$ wordt een overzicht gegeven van beschikbare technieken voor het herevalueren van de axillaire lymfeklieren na voorbehandeling met systemische therapie, bij patiënten met borstkanker waar bij diagnose sprake was van histologisch bewezen axillaire metastasering. Na evaluatie van 987 abstracts werden uiteindelijk 4 studies opgenomen in het review. In deze studies werden lichamelijk onderzoek, echografie 
van de axilla, MRI van de mamma, een FDG-PET-CT van het hele lichaam en een nomogram onderzocht om de patiënten met axillaire complete respons te selecteren. De kwaliteit van alle studies was beperkt door onder andere kleine steekproeven en het gebruik van verschillende definities van axillaire complete respons. Geconcludeerd werd dat er momenteel geen techniek voor handen is die accuraat de patiënten met een axillaire complete respons na systemische therapie kan identificeren.

Hoofdstuk 6 beschrijft de ontwikkeling en de diagnostische waarde van een nomogram op basis van standaard beschikbare parameters van 291 klinisch lymfeklier positieve patiënten met borstkanker om een voorspelling te kunnen doen van axillaire complete respons na voorbehandeling met systemische therapie. Het nomogram bleek redelijk nauwkeurig met een AUC van slechts 0.77 , maar dit bleek niet betrouwbaar genoeg om op basis van een hoge nomogram score af te zien van verdere axillaire behandeling bij de individuele patiënt.

Hoofdstuk 7 rapporteert over de diagnostische waarde van een gadofosveset versterkte axillaire MRI voor het (her-)evalueren van de axillaire lymfeklieren in een $2^{\mathrm{e}}$ cohort patiënten met borstkanker $(n=73)$. Over de gehele groep gemeten waren de diagnostische waarden van een standaard MRI en de gadofosveset versterkte MRI gelijkwaardig. Echter, nadat feedback was gegeven aan de beoordelende radiologen na 50 patiënten, werd een verbetering gezien van de diagnostische waarden in de laatste 23 patiënten. Met een gerapporteerde NPV van $94 \%$ in deze laatste groep, benadert een gadofosveset versterkte axillaire MRI de NPV van $97 \%$ van de algemeen geaccepteerde maar meer invasieve schilwachtklierprocedure.

Hoofdstuk 8 evalueerde het effect van onwetendheid van de ware pathologische axillaire lymfeklierstatus in een patiëntengroep $(n=303)$, met op voorhand gunstige tumorkenmerken, op het adviseren van adjuvante systemische behandeling. Terwijl $5 \%$ van de geïncludeerde patiënten micrometastatische en $10 \%$ macrometastatische ziekte had in hun axillaire lymfeklieren, veranderde de aanwezigheid van deze metastasen slechts in 1.0-3.6\% van de gevallen het adjuvante systemische behandelplan. De impact van de pathologische lymfeklierstatus op het adjuvante systemische advies is beperkt in deze klinisch lymfeklier negatieve patiënten groep met borstkanker.

In hoofdstuk 9 werd de hoeveelheid bijkomende stralingsdosis op de locatie van de schildwachtersklier geëvalueerd in een patiënten groep die werd behandeld met een lumpectomie gevolgd door bestraling van de gehele mamma. De locatie van deze schildwachtersklier werd vastgesteld door middel van een preoperatieve SPECT-CT en een post-operatieve radiotherapieplannings $\mathrm{CT}$, waarop een metalen clip zichtbaar was, die ter plaatste van de verwijderde schildwachtersklier werd achtergelaten tijdens de operatie. In 32 van de 42 patiënten bleek dat de locatie van de schildwachtersklier een 
electieve dosis radiotherapie ontving als de gehele mamma werd bestraald. Bestraling van de gehele mamma na een lumpectomie, in patiënten waarbij in de toekomst mogelijk de schildwachtersklier achterwegen gelaten zou kunnen worden, kan mogelijk bijdragen om een verhoging van het regionale recidief te voorkomen. 
Valorisation 


\section{Valorisation}

\section{Introduction}

Breast cancer is the most common cancer among women worldwide, with 1.38 million newly diagnosed breast cancer patients in 2008. The highest incidence rates are reported in Western countries and in the Netherlands 14,000 women are diagnosed each year with breast cancer. Due to its high incidence, breast cancer has an important societal and economic impact in the Netherlands. Health care costs related to breast cancer are estimated to be 696 million euros annually.

Survival rates in breast cancer are still increasing, with a current 5-year overall survival of $88 \%$, owing to better multidisciplinary treatment. Treatment consist of resection of the primary tumor and one or more axillary lymph nodes, and if indicated followed by a combination of hormonal-, chemo-, immuno- and/or radiotherapy. On the other hand, quality of life of survivors is affected by the morbidity caused by this treatment. Consequently, it is important to select patients that will benefit from the separate components of breast cancer treatment and to prevent exposure of patients to components of the treatment that do not positively affect their survival, while causing lifetime morbidity.

\section{Relevance of scientific results in this thesis}

This thesis addressed several topics on (re-)staging of axillary lymph nodes and the treatment of these nodes. For a long time the standard procedure to assess axillary lymph node status in breast cancer patients was axillary lymph node dissection (ALND). ALND is associated with significant short- and long-term morbidity. Most reported complications are seroma (15-75\%), upper extremity lymph edema (20\%), nerve injury $(55-75 \%)$ and reduced shoulder function (16\%). The psychological impact of lymphedema has been described to be as distressing as the initial diagnosis of breast cancer. Patients with breast cancer related lymphedema have been found to have a lower quality of life, a higher level of anxiety or depression, a higher likelihood of chronic pain and fatigue, and greater difficulty functioning socially and sexually compared to breast cancer patients without lymphedema.

In the past 15 years, the sentinel lymph node biopsy (SLNB) procedure has become the standard invasive technique to evaluate the nodal status in clinically node negative breast cancer patients. During a SLNB procedure, 1-2 axillary lymph node will be removed, compared to at least 10 axillary lymph nodes during an ALND. Complications known from the ALND still occur after SLNB, though less frequently; most reported are seroma, hematoma, lymphedema, numbness, tingling and pain. Severe lymphedema occurs in still $8 \%$ after a follow-up of 3 years. 
To avoid unnecessary morbidity related to the treatment of the axillary lymph nodes, we evaluated the use of axillary ultrasound as a selection tool to direct patients to an ALND of SLNB. We showed that with the standard use of axillary ultrasound, some patients will be overtreated with ALND, and thus unnecessary exposed to possible longterm morbidity of this procedure. Therefore, we advised that for the current daily practice, the use of axillary ultrasound should be discussed for every patient individually.

Form other studies we learned that the detection and surgical removal of every metastatic lymph node is not mandatory anymore, without compromising overall survival. This creates new opportunities to avoid even the less invasive SLNB procedure in patients without lymph node metastases. It is assumed that axillary ultrasound is probably inaccurate to select the patients with node negative breast cancer, since we showed that still $16 \%$ with a negative axillary ultrasound would have macro metastatic disease. After studying multiple nodal (re-)staging techniques, we found that a gadofosveset-enhanced axillary MRI has the highest potential as a noninvasive method for nodal (re-)staging in patients with breast cancer. The use of gadofosveset-enhanced axillary MRI can probably eliminate overtreatment and minimize the morbidity of any invasive nodal staging procedure in breast cancer. This is also of economic value; besides sparing the costs of a SLNB procedure, the related costs to relieve the morbidity can also be saved. Nonetheless, results on this technique first have to be confirmed in future studies, before it can be implemented in the treatment of new breast cancer patients.

\section{Target population}

The results of thesis are relevant for patients with new diagnosed breast cancer. Further, it offers more information on axillary nodal staging and treatment consequences for clinicians involved in the multidisciplinary treatment of patients with breast cancer.

\section{Innovation and Future}

The use of gadofosveset enhanced axillary MRI for nodal staging in breast cancer is innovative. This contrast agent was originally developed for imaging blood vessels. Gadofosveset is very effective fort his purpose, since it binds to albumin, and therefore remains in the vascular systemic for a prolonged period. Due to its affinity with albumin, it is also useful to image lymph nodes. Gadofosveset enhanced MRI has proven already is safety and efficacy in patients with rectal cancer. This thesis is the first that shows its also promising in breast cancer patients. Therefore, it is not unlikely that gadofosveset enhanced MRI could be useful in other tumor types, when the absence or presence of lymph node metastases determinates the treatment plan. 
Future research should be conducted to confirm the diagnostic performance of gadofosveset-enhanced MRI on patient level in a larger group of patients with breast cancer, preferably in a multi-center setting. Ultimately, clinical outcome studies are needed to understand whether an NPV of $94 \%$ is oncologically safe to replace the current SLNB and ALND. The slightly lower NPV gadofosveset-enhanced axillary MRI could theoretically increase the risk of regional recurrences, yet this disadvantage could well balance against the treatment related morbidity associated with SLNB and ALND. 
List of publications 


\section{List of publications}

De Mini Suffering State Exam (MSSE) onderzocht in een Nederlands verpleeghuis. Schols RM, Schipper RJ, Brabers A, Schols JMGA. Tijdschr Verpleeghuisgeneeskd 2003; 27 (5): pp. 14-18.

Anatomical evaluation of the internal mammary vessels based on magnetic resonance imaging (MRI). Tuinder S, Dikmans R, Schipper RJ. Boetes C. Lobbes M., Peeters W, van der Hulst R. J Plast Reconstr Aesthet Surg, 2012 Oct;65(10):1363-7.

Noninvasive Nodal Staging in Patients With Breast Cancer Using GadofosvesetEnhanced Magnetic Resonance Imaging: A Feasibility Study. Schipper RJ, Smidt ML, van Roozendaal LM, Castro CJ, de Vries B, Heuts EM, Keymeulen KB, Wildberger JE, Lobbes MB, Beets-Tan RG. Invest Radiol. 2013 Mar;48(3):134-9.

Bilateral analysis of the cross-sectional area of the internal mammary arteries and veins in patients with and without breast cancer on breast magnetic resonance imaging. Schipper RJ, Lobbes MB, Dikmans RE, Beets-Tan RG, Smidt ML, Boetes C. Insights Imaging. 2013. Apr;4(2):177-84.

The role of magnetic resonance imaging in assessing residual disease and pathologic complete response in breast cancer patients receiving neoadjuvant chemotherapy: a systematic review. Lobbes MB, Prevos R, Smidt M, Tjan-Heijnen VC, van Goethem M, Schipper RJ, Beets-Tan RG, Wildberger JE. Insights Imaging. 2013 Apr;4(2):163-75.

Is repetition of the contralateral mammogram of patients referred for unilateral findings from the Dutch Breast Cancer Screening necessary? Castro CJG, Schipper RJ, van Roozendaal LM, van Goethem M., Lobbes MBI, Smidt ML. ABCR, Pub date Sept 23, 2013. DOI: $10.4236 / a b c r .2013 .24023$

Oral pemphigus as first sign of an inflammatory myofibroblastic tumor in an 18-year old male. Schols RM, Beets GL, RiedI RG, Schipper RJ. BMJ Case Reports. 2013 Oct 28;2013.

A women with a history of colorectal carcinoma presenting with an abdominal mass. Schipper RJ, Stassen LP, Smidt ML, Schols RM. BMJ Case Reports. 2013 Nov 5;2013.

No increase in local recurrence rate in breast cancer patients treated with skin-sparing mastectomy followed by immedicatie breast reconstructuion. van Mierlo DR, Lopez Penha TR, Schipper RJ, Martens MH, Serroyen J, Lobbes MB, Heuts EM, Tuinder S, Smidt ML. Breast. 2013 Dec;22(6):1166-70. 
Axillary ultrasound for preoperative nodal staging in breast cancer patients: Is it of added value? Schipper RJ*, van Roozendaal LM*, de Vries B, Pijnappel RM, Beets-Tan RGH, Lobbes MBI, Smidt ML. The Breast. 2013 Dec;22(6):1108-13.

Even goed, met minder: behandeling van de oksel bij mammacarcinoompatienten. LM van Roozendaal, RJ Schipper, M Moossdorff, LJA Strobbe, ML Smidt. Kanker Breed, volume 5, nummer 2, Juli 2013

The impact of the pathological lymph node status on adjuvant systemic treatment recommendations in clinically node negative breast cancer patients. van Roozendaal LM*, Schipper RJ*, van de Vijver KK, Haekens CM, Lobbes MBI, Tjan-Heijnen VCG, de Boer M, Smidt ML. Breast Cancer Res Treat. 2014 Fev;143(3):469-76.

A model to predict pathologic complete response of axillary lymph nodes to neoadjuvant chemo(-immuno)therapy in patients with clinically node positive breast cancer Schipper RJ, Moossdorff M, Nelemans PJ, Nieuwenhuijzen GAP, de Vries B, Strobbe LJA, Roumen RHM, van den Berkmortel F, Tjan-Heijnen VCG, Beets-Tan RGH, Lobbes MBI, Smidt ML. Clinical Breast Cancer. 2014 Oct;14(5):315-22.

Inconsistent selection and definition of local and regional endpoints in breast cancer trials. Moossdorff M, van Roozendaal LM, Schipper RJ, Strobbe LJ, Voogd AC, TjanHeijnen VCG, Smidt ML. Br J Surg. 2014 Dec;101(13):1657-65.

Kleine kans op uitgebreide lymfekliermetastasering na een negatieve echografie van de axilla bij mammacarcinoompatienten in Nederland. Duijsens GH, van Roozendaal LM, Schipper RJ, Paiman EH, Siesling S, Pijnappel RM, Lobbes MB, Smidt ML. Nederlands tijdschrift voor Oncologie. 2014;11:268-73.

Axillary response monitoring after neoadjuvant chemotherapy in breast cancer: can we avoid the morbidity of axillary treatment? Vugts G, Schipper RJ, Maaskant-Braat AJ, Smidt ML, Nieuwenhuijzen GA. Ann Surg. 2014 Nov 17.

The diagnostic performance of dedicated axillary T2- and diffusion weighted MRI for nodal staging in breast cancer. RJ Schipper, M Paiman, RGH Beets-Tan, PJ Nelemans, B de Vries, EM Heuts, KK vd Vijver, KB Keymeulen, B Brans, ML Smidt, MBI Lobbes. Radiology. 2015 May;275(2):345-55.

Three-Dimensional breast radiotherapy and the elective radiation dose at the sentinel lymph node site in breast cancer. LM van Roozendaal, RJ Schipper, EM Heuts, KBMI Keymeulen, J Buijsen, B Brans, RGH Beets-Tan, L Boersma, ML Smidt. Ann Surg Oncol 2015 Feb 24. Epub ahead of print. 
Noninvasive nodal restaging in clinical node positive breast cancer patients after neoadjuvant systemic therapy: a systematic review. RJ Schipper, M Moossdorff, ML Smidt, RGH Beets-Tan, MBI Lobbes. Eur J Radiol. 2015 Jan;84(1):41-7.

The role of MRI in axillary lymph node imaging in breast cancer patients: a systematic review. Kuijs VJ, Moossdorff M, Schipper RJ, Beets-Tan RG, Heuts EM, Keymeulen KB, Smidt ML. Lobbers MB. Insights Imaging. 2015 Apr;6(2):2013-15

The diagnostic performance of sentinel lymph node biopsy in pathologically confirmed node positive breast cancer patients after neoadjuvant systemic therapy; a systematic review and meta-analysis. Van Nijnatten TJ, Schipper RJ, Lobbes MB, Nelemans PJ, Beets-Tan RG, Smidt ML. Accepted, European Journal of Surgical Oncology

The diagnostic performance of gadofosveset-enhanced axillary MRI for nodal (re-)staging in breast cancer patients: can the initial promising results be reproduced? Schipper RJ, Smidt ML, van Roozendaal LM, Nelemans PJ, Paiman M, Brans BT, Moossdorff M, de Vries B, Keymeulen KB, Wildberger JE, Lobbes MB, Beets-Tan RG. Submitted 
Dankwoord 


\section{Dankwoord}

Terugkijkend op de totstandkoming van dit proefschrift kan ik niet anders dan concluderen dat een zeer grote groep mensen hier direct en indirect bij betrokken is geweest. Hiervoor allereerst een hele grote DANK JE WEL!

Ondanks het risico te lopen belangrijke personen te vergeten, wil ik het niet nalaten om enkelen ook bij naam te bedanken. Maar dat niet zonder eerst mijn bewondering uit te spreken voor de patiënten die, zonder eigen belang in een moeilijke tijd van hun leven, hebben geparticipeerd in de verschillende prospectieve onderzoeken. Zonder patiënten zoals jullie, zouden we niet in staat zijn om de behandeling van het mammacarcinoom naar een steeds hoger niveau te tillen.

Dit proefschrift was er nooit gekomen zonder prof. dr. Carla Boetes. Zij was degene die het wetenschappelijke vuurtje in mij heeft weten te ontbranden tijdens mijn keuze coschap op de afdeling radiologie. Met jouw grote kennis van zaken en charme wist je mij in een korte tijd te interesseren om als $1^{\mathrm{e}}$ promovendus van jou in Maastricht aan de slag te gaan. Een nieuwe onderzoekslijn met het stadiëring van de axillaire lymfeklieren met behulp van een MRI scanner als doel. Helaas heb jij het officiële begin van dit ambitieuze onderzoek nooit mee mogen maken wegens jouw plotselinge overlijden.

Prof. dr. Beets-Tan, beste Regina, ik werd na het overlijden van Carla in jouw "schoot" geworpen. Je was voor mij een logische en meer dan waardige vervanger om leiding te geven aan het tot stand komen van dit proefschrift. Vanaf moment een heb ik me altijd welkom gevoeld! Jouw kennis met betrekking tot het stadieringen van de lymfeklieren met MRI bij patiënten met een rectumcarcinoom is van onschatbare waarde geweest. Daarnaast met jouw scherpe en kritische blik werd elk onderzoeksvoorstel en manuscript beter. Ik bewonder je drive om steeds maar weer nieuwe 'tools' te ontwikkelen en te testen, om de "clinici" van meer en betrouwbaardere informatie te voorzien, zodat de patiënt een steeds betere en individuelere behandeling kan krijgen! Ik wens je heel veel succes in het Amsterdamse, en hoop ook in de toekomst nog veel van je te leren!

Dr. Smidt, beste Marjolein, terwijl jij op Bonaire was werd ik door dr. Esther Heuts aangenomen om bij jouw mijn $6^{\mathrm{e}}$ jaar van de opleiding geneeskunde te volbrengen. $\mathrm{Nu}$ meer dan 5 jaar later kan ik alleen maar terugkijken op een fantastische tijd! Wie had toen kunnen bedenken dat ik jouw eerste copromotor "kindje" zou worden. Ik wil je heel erg bedanken voor al je inspanningen met betrekking tot dit onderzoek. Zonder jouw enthousiasme en doorzettingsvermogen waren we nooit in staat geweest dit onderzoek te volbrengen! Je mag trots zijn op wat jij allemaal hebt weten klaar te 
spelen, weinige zullen je dit nadoen! Ik kijk ernaar uit om onze samenwerking ook in de toekomst voort te zetten en naar de vele gezellige diners en BBQ's bij jullie thuis!

Dr. Lobbes, beste Marc, wat een rollercoaster is het de afgelopen jaren geweest! Na het overlijden van Carla heb jij de zware last op je genomen om zorg te dragen voor de mammadiagnostiek binnen het MUMC+. Dit is je naar mijn bescheiden mening fantastisch gelukt. Daarnaast ben je ook nog in staat geweest om de wetenschappelijke trein door te laten rijden. We kunnen inmiddels wel stellen dat dit ook nog eens geen normale trein is, maar een echte TGV! Naast je "eigen" onderzoeksprojecten stond je altijd voor mij klaar. Er was ondanks jouw drukke agenda altijd tijd om in het "mammohok" bij te praten en nieuwe onderzoek ideeën te bespreken. Je doelgerichte en snelle manier van werken was zeer plezierig!

Prof. dr. Wildberger, beste Joachim. Ook $u$ wil ik persoonlijk bedanken. Je vastberadenheid om de onderzoekslijn van Carla door te laten lopen, heeft zeer zeker bijgedragen aan het volbrengen van dit proefschrift. Bij vragen en problemen kon ik altijd snel terecht om met een degelijk advies weer te vertrekken. Dank voor alles!

Dit proefschrift had niet tot stand kunnen komen zonder de medewerking van de mammacare verpleegkundigen en verpleegkundig specialisten op de polikliniek van het oncologiecentrum; Elly, Conny, Janine en Christel, heel erg bedankt! Daarnaast natuurlijk ook veel dank voor de mammachirurgen in het MUMC+! Zonder jullie bijdrage was het nooit gelukt om de vele onderzoeken tot een goed einde te brengen!

Daarnaast natuurlijk veel dank aan alle radiologielaboranten die met veel toewijding de vele MRI's scan hebben gemaakt waarop dit proefschrift grotendeels is gebaseerd. Speciale dank hierbij voor Dagmar, Eslina en Shahla. Jullie stonden altijd klaar om weer eens een test MRI te maken, of om afspraken om te gooien, zodat net die ene patiënt toch nog op tijd een MRI van de axilla kon krijgen. Super bedankt voor jullie inzet!

Ook ben ik veel dank verschuldigd aan de afdeling pathologie van het MUMC+. In het bijzonder dr. Koen vd Vijver en Bart de Vries. Hartelijk dank voor jullie geduld om elke okselklierdissectie zorgvuldig te verwerken, zodat wij in staat waren om een perfecte match te maken met de MRI scans!

Graag wil ook het de secretariaten van de afdelingen chirurgie en radiologie bedanken; in het bijzonder Elfie en Sabeth!

Speciale dank gaat ook uit naar de vele semi-artsen die betrokken zijn geweest bij de verschillende onderzoeken die hebben geleid tot dit proefschrift! Daarnaast natuurlijk 
ook mijn collega mammacarcinoom onderzoeksters Lori, Martine en Tiara. Het was een plezier om met jullie samen te werken!

Ook wens ik mijn opvolgers Briete en Thiemo heel veel succes! Ik ben ervan overtuigd dat jullie het huidige onderzoek fantastisch doorzetten en met de vele nieuwe ideeën ook tot mooie proefschriften zullen komen!

Voor zover nog niet genoemd, dank aan alle coauteurs van de verschillende hoofdstukken in dit proefschrift. Zonder jullie kritische blik en adviezen zouden de manuscripten niet van die kwaliteit zijn worden waardoor ze nu bijna allemaal in gerenommeerde journals gepubliceerd zijn.

Natuurlijk wil ik ook de leden van de beoordelingscommissie, prof. dr. G.L. Beets, prof. dr. M. van Goethem, prof. dr. M.F. von Meyenfeldt, prof. dr. E.J. Rutgers, prof. dr. H.J. Rutten en dr. R.M. Mann, bedanken voor het beoordelen van dit proefschrift, en hun tijd en interesse om dit proefschrift te bediscussiëren tijdens de verdediging. Ook bedank ik het overig lid van de promotiecommissie, dr. R.M. Pijnappel, voor het optreden als opponent tijdens de verdediging.

Het leven van arts-onderzoeker kent natuurlijk naast de wetenschappelijke kant ook vele andere aspecten. Desondanks dat er geen directe relatie bestaat de inhoud en volbrengen van dit proefschrift, wil ik ook enkele van hen persoonlijk bedanken!

Oud-collega's van de onderzoeksafdelingen chirurgie en radiologie binnen het MUMC+. Bedankt voor jullie interesse in mijn onderzoek en de gezelligheid!

Speciale dank gaat ook uit naar de fantastische groep chirurgen en arts-assistenten van het Catharina Ziekenhuis in Eindhoven. Zonder jullie steun en enthousiasme was ik nooit instaat geweest om zo snel in opleiding te zijn tot chirurg en ook nog eens dit proefschrift af te ronden!. Ik voel me thuis in 't Cathrien, dank daarvoor!

Het leven is een stuk aangenamer met vele stapavonden, feestjes en festivals. Daarom dank voor alle vrienden en vriendinnen in het Maastrichtse,, Amsterdamse, Utrechtse, Eindhovense en elders. Speciale dank ook voor de Oelbert vrienden groep. Onze sporadische avondjes zijn altijd legendarisch! Laten we dat vooral voortzetten!

Daarnaast mag natuurlijk HJC Uitzonderring niet ontbreken in dit dankwoord. Heeren van deze illustere club, - Tom, Broos, Jeroen, Valentijn, Rutger, Tim, Maarten, Bart, Felix, Wisse - wat een mooie studententijd hebben we gehad in het Maastrichtse. Alles mag en kon, niks was te gek! Laten we daar, ook na 10 jaar vriendschap, vooral mee doorgaan! 
Een speciaal woordje nog voor mijn beide paranifmen; Rutger, alias "de prof". Vanaf de $2^{\mathrm{e}}$ klas op het St-Oelbert in Oosterhout heb ik jou echt leren kennen. Daarna zijn onze wegen een lange onafscheidelijke geweest; dezelfde studie in dezelfde stad, daarnaast nog eens de zelfde studentenvereniging en jaarclub. En dat was nog niet eens alles; ondanks dat we niet tegelijk begonnen met ons promotieonderzoek kwamen we toch weer op dezelfde kamer terecht op de UNS 50. Wie verzint zoiets? Mede dankzij onze vriendschap en samenwerking, en niet te vergeten de vers gemalen koffie die letterlijk tussen ons in stond, werd er volop "productie" gedraaid op de "office". Wat een fantastische school-, studenten- en onderzoekerstijd hebben we gehad! Ik ben ook super trots op je dat je je droom hebt waargemaakt, in opleiding tot plastisch chirurg!

Daarnaast natuurlijk Tom, alias Poppelini. Vrienden sinds de start van onze studententijd. Ik vind het geweldig dat jij op deze bijzondere dag ook paranimf wil zijn. Ik hoop dat onze vriendschap eeuwig blijft bestaan, en dat we ooit nog eens gezamenlijk gaan skiën ;)

Marjolein en Lisette, alias Lein en Kip, mijn zus en zusje. Het is altijd weer een feest om bij jullie over de vloer te komen, of om samen aan de bar te drinken. Dat laatste doen we eigenlijk te weinig :P. Ik ben trots op jullie!

Oma, wat ben ik trots op u. 85 lentes jong en nog altijd zelfstandig! Ik moet het nog maar zien te halen. Samenzijn met $u$ is altijd leuk en staat garant voor gezelligheid!

Mam en Dad, bedankt voor alles! Mede dankzij jullie liefde, steun, adviezen en vrijheid sta ik waar ik nu sta. Ik kan me geen beter leven wensen. Ontelbaar veel dank! 
Curriculum vitae 


\section{Curriculum vitae}

Robert-Jan Schipper was born on October 1st, 1986 in Oosterhout (NB), The Netherlands. In 2005 he graduated from the St. Oelbert Gymnasium in Oosterhout (NB), The Netherlands. Immediately thereafter he started his medical training at the Faculty of Medicine at the Maastricht University. During this study, he attended his elective internship at the radiology department of the Maastricht University Medical Center+ under supervision of drs. S. Gommers. During this internship Prof. dr. Boetes encouraged Robert-Jan to participate in her research group. The $6^{\text {th }}$ year of his medical training was completed with a combined clinical and scientific training program at the departments of surgery and radiology of the Maastricht University Medical Center+. Consequently, after obtaining his medical degree in 2011, he officially started as PhD student under supervision of Prof. dr. Beets-Tan, dr. Smidt and dr. Lobbes. The main goal of his PhD project was to study the opportunities of a more tailored made treatment plan for each breast cancer patient, in order to minimize the morbidity of the breast cancer survivor without compromising overall survival. The results are described in this thesis.

In April 2014 Robert-Jan started as senior house officer (ANIOS) at the department of surgery at the Catharina Hospital Eindhoven. In January 2015 he started his surgical training program at the Catharina Hospital Eindhoven under supervision of dr. G.A.P. Nieuwenhuijzen and dr. S.W. Nienhuijs. 
